\title{
Potassium Double Tungstate Waveguides with High Ytterbium Concentration for Optical Amplification
}

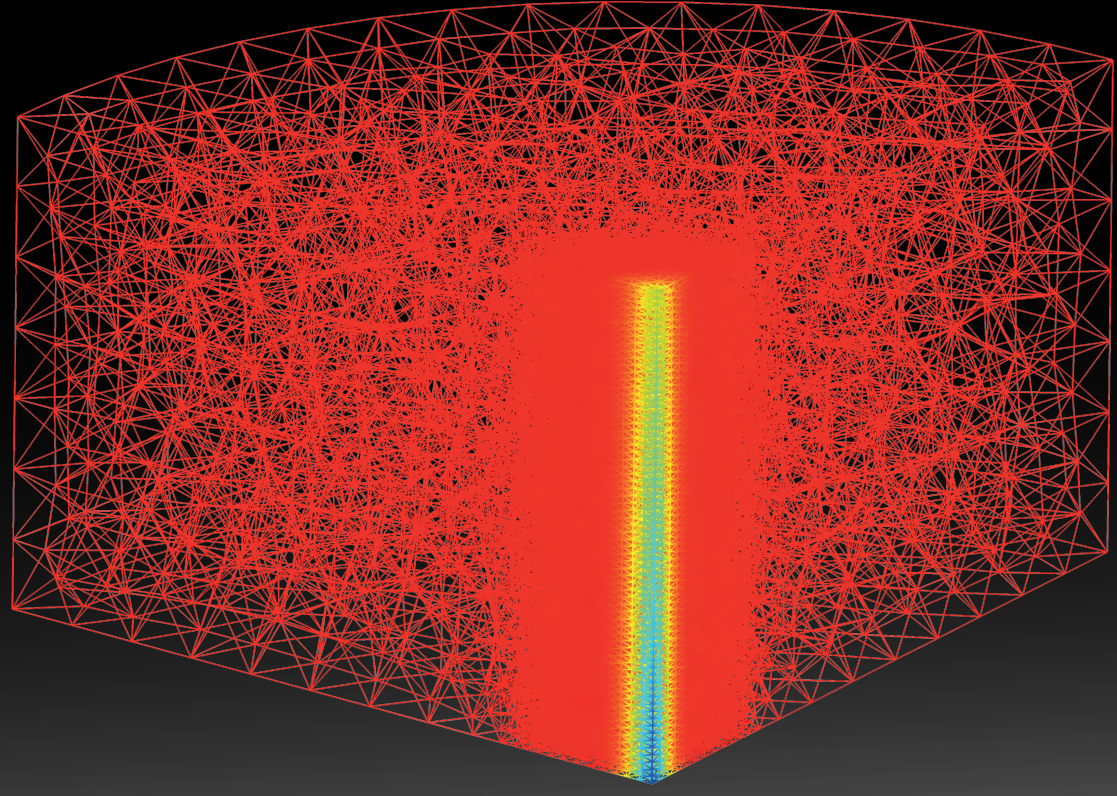

Yean-Sheng Yong 


\section{POTASSIUM DOUBLE TUNGSTATE WAVEGUIDES WITH HIGH YTTERBIUM CONCENTRATION FOR OPTICAL AMPLIFICATION}

Yean-Sheng Yong 


\section{Graduation committee:}

Chairman and Secretary:

Prof. Dr. Ir. J. W. M. Hilgenkamp

Supervisor:

Prof. Dr. M. Pollnau

Prof. Dr. J. L. Herek

\section{Co-supervisor:}

Assoc. Prof. Dr. S. M. García-Blanco

Members:

Prof. Dr. C. Denz

Assoc. Prof. Dr. J. J. Carvajal

Prof. Dr. K. -J. Boller

Prof. Dr. L. K. Nanver
University of Twente

University of Twente

University of Twente

University of Twente

University of Münster

Universitat Rovira i Virgili

University of Twente

University of Twente

The research described in this thesis was carried out at the Integrated Optical MicroSystems (IOMS) Group, Faculty of Electrical Engineering, Mathematics and Computer Science, and Optical Sciences (OS) Group, Faculty of Science and Technology, MESA+ Institute for Nanotechnology, University of Twente, P.O. Box 217, 7500 AE Enschede, The Netherlands.

The research was financially supported by the Dutch Technology Foundation STW (project \#11689 “Integrated Waveguide Amplifiers for Optical Interconnects”).

Cover design: Three dimensional visualization of optical amplification in potassium double tungstate waveguide layer as a signal beam propagates from the top surface to the bottom surface. The model has a radius equivalent to about twenty times the optical beam's radius and only a quarter of the body is shown. The intensity of the signal beam is represented by the color spectrum (red being lowest and blue being highest). The wire frame is generated based on finite element mesh grids.

Copyright (C) 2017 by Yean-Sheng Yong, Enschede, The Netherlands

ISBN: 978-90-365-4356-9

DOI: $10.3990 / 1.9789036543569$

URL: https://doi.org/10.3990/1.9789036543569 


\title{
POTASSIUM DOUBLE TUNGSTATE WAVEGUIDES WITH HIGH YTTERBIUM CONCENTRATION FOR OPTICAL AMPLIFICATION
}

\section{DISSERTATION}

\author{
to obtain \\ the degree of doctor at the University of Twente, \\ on the authority of the rector magnificus, \\ prof. dr. T. T. M. Palstra
}

on account of the decision of the graduation committee,

to be publicly defended

on Thursday the $22^{\text {nd }}$ of June 2017 at 12:45

by

\section{Yean-Sheng Yong}

born on the $28^{\text {th }}$ of August 1984

in Kuala Lumpur, Malaysia 
This dissertation is approved by:

Prof. Dr. M. Pollnau

(supervisor)

Prof. Dr. J. L. Herek

(supervisor)

Assoc. Prof. Dr. S. M. García-Blanco

(co-supervisor) 


\section{Table of Content}

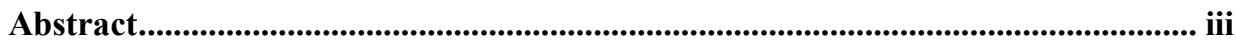

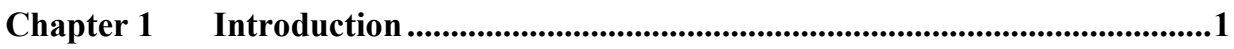

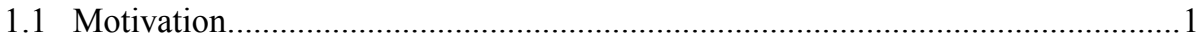

1.2 Rare-earth-ion-doped gain materials............................................................2

1.3 State-of-the-art waveguide amplifiers.........................................................4

1.4 Ytterbium-activated potassium double tungstate amplifiers ............................... 7

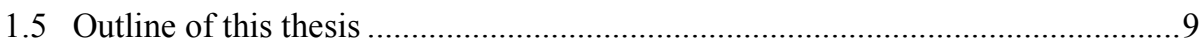

Chapter 2 Theoretical background ..................................................................11

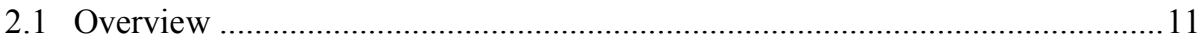

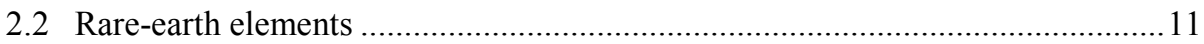

2.3 Interaction of light and matter................................................................ 13

2.3.1 Absorption, spontaneous emission, and stimulated emission.................13

2.3.2 Lifetime and effective cross-sections ................................................. 15

2.3.3 Gain and loss in optically active media.............................................. 19

2.3.4 Theories for determining effective cross-sections ............................... 19

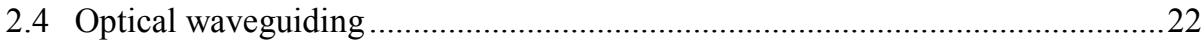

2.4.1 Waveguides based on potassium double tungstates ............................23

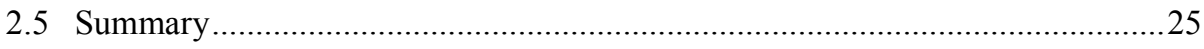

Chapter $3 \quad \mathbf{Y b}^{3+}$-activated potassium double tungstates .....................................27

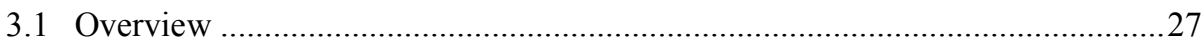

3.2 Properties of $\mathrm{Yb}^{3+}$-doped potassium double tungstate bulk crystals ..................27

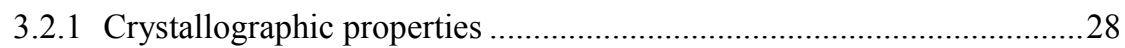

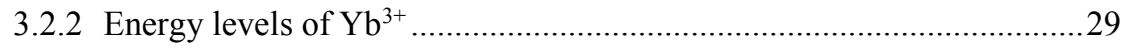

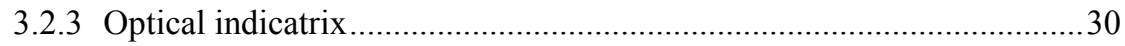

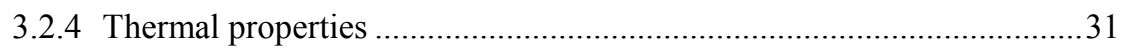

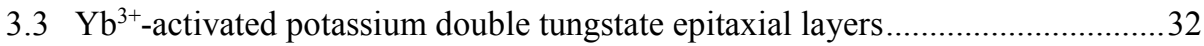

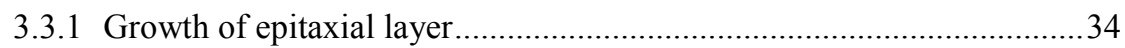

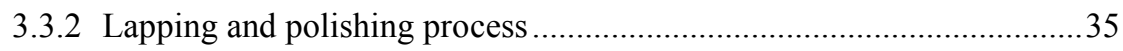

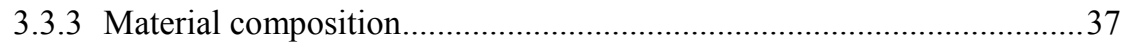

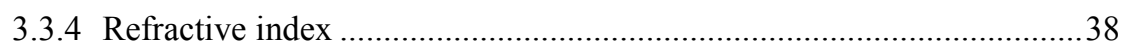

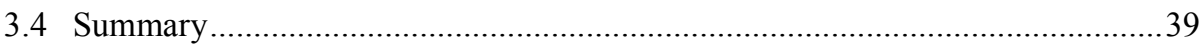

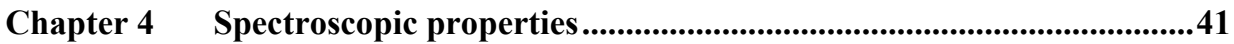

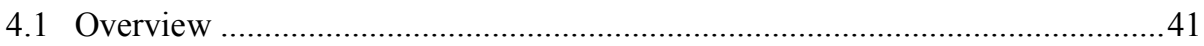

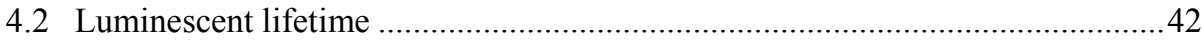

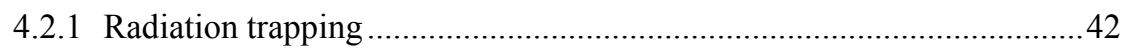

4.2.2 Lifetime measurement using confocal method...................................44 
4.2.3 Concentration dependence of luminescence lifetime........................... 46

4.2.4 Power dependence of luminescence lifetime ..................................... 49

4.3 Absorption and emission cross-sections ..................................................... 51

4.3.1 Absorption measurement ............................................................... 51

4.3.2 Factors affecting the measurement results ........................................ 53

4.3.3 Evaluation of transition cross-sections................................................. 55

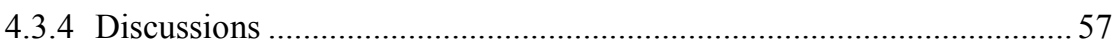

4.4 Temperature dependence of spectroscopic parameters....................................... 58

4.4.1 Temperature dependence of luminescent lifetime ................................5 59

4.4.2 Temperature dependence of cross-sections......................................... 60

4.4.3 Temperature dependence of major absorption lines ............................. 61

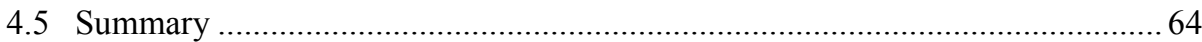

Chapter 5 Material gain in thin film .......................................................................6 67

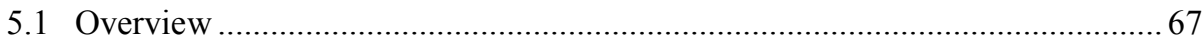

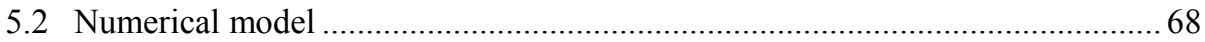

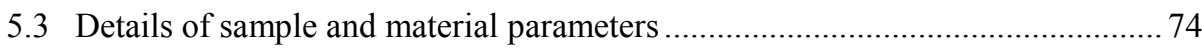

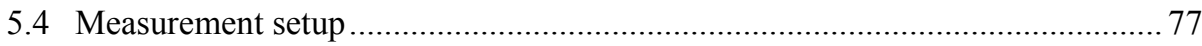

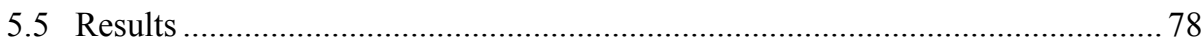

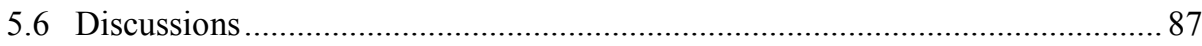

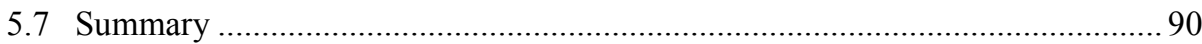

Chapter 6 Conclusions........................................................................................... 91

Appendix Review on waveguide amplifiers.................................................97

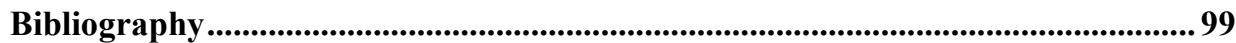

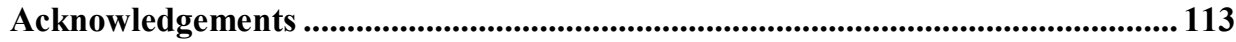

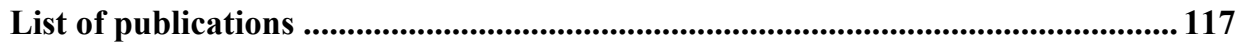




\section{Abstract}

In this thesis, the research work concerning high ytterbium concentration potassium double tungstate waveguides catered for optical amplification purpose is presented. The scope of the research work includes the investigation of spectroscopic and optical gain properties in epitaxy layers with concentration of trivalent ytterbium $\left(\mathrm{Yb}^{3+}\right)$ up to 76 at. $\%$, which is equivalent to $\mathrm{Yb}^{3+}$ density of $\sim 5 \times 10^{21} \mathrm{~cm}^{-3}$.

In order to obtain accurate luminescence lifetime value in epitaxy layers with high $\mathrm{Yb}^{3+}$ concentration, a novel confocal measurement setup is proposed to mitigate the radiation trapping effect which elongates the measured lifetime. By performing measurement on samples with various $\mathrm{Yb}^{3+}$ concentrations under low launched pump power, it is shown that the lifetime values obtain from the proposed setup are close to the consensus values in the literature measured using other more involved procedures. Besides, it is confirmed that concentration dependent lifetime quenching on high $\mathrm{Yb}^{3+}$ concentration potassium double tungstate epitaxial layers is rather weak. The lifetime measured from sample with highest $\mathrm{Yb}^{3+}$ concentration of 76 at.\% is $222 \mu \mathrm{s}$ as compared to the lifetime of $245 \mu$ s obtained from sample with only 1.2 at.\% $\mathrm{Yb}^{3+}$ concentration. In addition, it is found that the measured power dependent luminescence decay curves exhibit non-exponential decay. Such behavior is associated with energy transfer upconversion (ETU) process and an ETU coefficient of $1.3 \times 10^{-18} \mathrm{~cm}^{3} / \mathrm{s}$ is quantified based on the measured decay curves.

As the epitaxy layers exhibit unusually high amount of $\mathrm{Yb}^{3+}$, determination of transition cross-sections in these media is not trivial. Systematic studies have been performed to investigate the impact of two factors, namely the polarization disorientation and the stray light in the measurement system, on measured absorption. The results reveal that these factors may contribute to under-estimation of peak transition cross-sections. Carefully evaluated transition cross-sections in epitaxial layers with 57 at.\% and 76 at. $\% \mathrm{Yb}^{3+}$ are found to be comparable to those of bulk $\mathrm{Yb}^{3+}$ activated potassium double tungstate crystals. The peak absorption and emission crosssections for both samples are $\sim 1.3 \times 10^{-19} \mathrm{~cm}^{2}$ and $\sim 1.6 \times 10^{-19} \mathrm{~cm}^{2}$, respectively, at the central transition line close to $981 \mathrm{~nm}$ wavelength.

The temperature dependence of luminescence lifetime and transition crosssections on sample with 57 at. $\% \mathrm{Yb}^{3+}$ is investigated in this work. It is believed that this is the first experimental report on the temperature dependence of spectroscopic properties in $\mathrm{Yb}^{3+}$-activated potassium double tungstates. The measurement results show negligible dependency of lifetime to the temperature. On the other hand, the cross-section spectra change drastically with the increase of temperature. A reduction of 
peak absorption cross-section at $\sim 981 \mathrm{~nm}$ wavelength by $52 \%$ has been observed when the temperature rises from $20{ }^{\circ} \mathrm{C}$ to $170{ }^{\circ} \mathrm{C}$. Such a strong temperature dependence prompted further investigation on the temperature dependence of the absorption peaks at $\sim 932 \mathrm{~nm}$ and $\sim 981 \mathrm{~nm}$, which correspond to the pump and signal wavelengths of the amplifiers concerned in this work. It is found that the temperature dependence of these absorption peaks is caused by two reasons: the fractional population at the starting Stark level and the linewidth of the respective transition at the given temperature. A simple expression which relates these two factors to the observe temperature dependence of absorption peaks is derived based on a fundamental theoretical analysis. The results calculated from this expression are in good agreement with the experimental observation.

The pump absorption and optical gain in epitaxial layer with 57 at.\% $\mathrm{Yb}^{3+}$ are studied by launching pump and signal beams perpendicularly to the layers. Besides, a numerical model which includes pump-induced heating effects, ETU process, and quenched ions is established. Comparison of experimental and modeled pump absorption results shows that the pump absorption is not saturated even after accounting for ETU process. The nonsaturable pump absorption is possibly due to presence of rapidly quenched ions which are not detected under typical spectroscopic measurements. Numerical calculations are performed to investigate the influence of ETU process and quenched ions on pump absorption, heating of pumped region, and signal gain. Both effects are found to lead to increase absorption of pump power, higher heat generation within the pump region, and lower signal gain. By incorporating both ETU process and quenched ions in the numerical model, experimental results of pump absorption and pump-probe signal gain can be explained. Net gain value of $2.62 \mathrm{~dB}$ $(817 \mathrm{~dB} / \mathrm{cm})$ is achieved in $32 \mu \mathrm{m}$ thick epitaxial layer without any thermal management. It is shown that localized heating and non-ideal inversion condition limited the experimentally observed gain. The luminescence spectra within both near infrared and visible wavelength range show broadened emission peaks with increase of launched pump power, hence confirming that significant amount of heat could be generated within the pump region.

In overall, the work described in this thesis provide advances in understanding the characteristics of high $\mathrm{Yb}^{3+}$ concentration potassium double tungstate waveguide layers. The experimental results show that favorable spectroscopic properties are retained in the epitaxial layers. Nevertheless, additional effects such as ETU process, quenched ions, and localized heating within the pumped region have also been discovered and analyzed. Particularly, elevated temperature on the gain medium would severely affect the absorption and emission behavior. The investigation of optical gain and luminescence spectra shows that the thermal effects play a role in high active ion concentration and intensely pumped amplifier. 


\section{Chapter 1 Introduction}

\subsection{Motivation}

Rare-earth-ion-doped fiber amplifier is a key enabling device for modern telecommunication systems. It allows all-optical amplification in fiber network without electrical-to-optical or optical-to-electrical signal conversion, hence greatly eases the transmission speed bottleneck introduced by conventional electrical amplifier. Due to its many favorable properties, such as high saturation output power, high gain over broad wavelength range, and high bit rate signal amplification, the fiber amplifier has become an indispensable device for long haul communication systems.

With the ever increasing demand on data transmission rate, the use of optical interconnects is no longer restricted to long distance communication network. Metropolitan scale fiber network is commonly available now. Optical interconnects are also widely adopted for short reach rack-to-rack communication links within data centers. With the advent of board scale polymer waveguide [1,2], high modulation bandwidth vertical cavity surface emitting laser (VCSEL) [3-5] and compact CMOS transceiver module [6], board level optical interconnect is now a viable option for data centers and high performance computer systems [7]. Recent advancements on propagation loss reduction in InP-based passive waveguide [8] as well as hybrid integration schemes used to combine amplifier, laser, photodiode, or nonlinear material with passive waveguide [9-12] are paving way for advanced photonics integrated circuits (PIC) with increasing complexity.

Over the years, chip scale rare-earth-ion-doped amplifier, which is known as rareearth-ion-doped waveguide amplifier, with device length significantly shorter than fiber amplifier has been developed. Rare-earth-ion-doped waveguide amplifier is promising for emerging board level optical interconnects and PICs with limited device footprint. Besides, it offers many advantages as compared to fiber amplifier. For instance, existing lithography equipment and infrastructure used in microelectronics industry can be applied to fabricate and process a large number of rare-earth-ion-doped waveguide amplifiers on the same chip. Rare-earth-ion-doped waveguide amplifier can be integrated with passive photonic circuit using monolithic scheme [13] or hybrid flipchip bonding scheme [14, 15]. These integration schemes significantly reduce the production cost in comparison to conventional butt coupling approach which requires end-face polishing and active alignment. Integrated rare-earth-ion-doped waveguide amplifier can be used to boost weak signal within PIC and to compensate optical loss in 
1-to- $N$ splitter [16], non-linear waveguide [17], or plasmonic waveguide [18]. That said, the signal output power and gain of rare-earth-ion-doped waveguide amplifier are lagging behind its fiber counterpart [19] and typical device length of rare-earth-iondoped waveguide amplifier is longer than that of semiconductor amplifier ( $\sim 10$ 's cm vs $\sim$ few $\mathrm{mm}$ ), hence further development on waveguide amplifier is still needed.

In this thesis, high ytterbium concentration potassium double tungstate waveguide layers are investigated in order to realize small footprint and high gain waveguide amplifier. Prior to this work, waveguide amplifier based on 47.5 at.\% trivalent ytterbium $\left(\mathrm{Yb}^{3+}\right)$ potassium double tungstates with $\sim 17 \mathrm{~dB}$ net gain (i.e. signal enhancement deducted by total losses experienced by the signal in the material) had been reported [20]. With a device length as short as $180 \mu \mathrm{m}$, the corresponding net gain per unit length for the potassium double tungstate waveguide amplifier is $935 \mathrm{~dB} / \mathrm{cm}$ [20], which is at least an order of magnitude higher than any other rare-earth-ion-doped materials and comparable to the modal gain of semiconductor amplifier [9, 21]. The focus of this work is to examine the spectroscopic and gain properties of potassium double tungstates epitaxial layers with $\mathrm{Yb}^{3+}$ concentration exceeding 50 at.\%.

\subsection{Rare-earth-ion-doped gain materials}

The incorporation of rare-earth ions in dielectric material allows absorption and emission of photons with certain wavelengths. The term 'doped' is commonly used as the amount of rare-earth ions included in the material is usually small. The optical response on the rare-earth-ion-doped material is depending on the type of active ion included and the host material itself. Figure 1.1 shows the simplified energy level diagrams of several rare-earth ions commonly used for optical amplification. The arrows in the figure show the relevant transitions labeled with indicative pump and signal wavelengths for the respective rare-earth-ion-doped material.

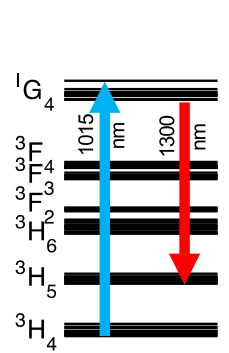

(a) Praseodymium $\left(\mathrm{Pr}^{3+}\right)$

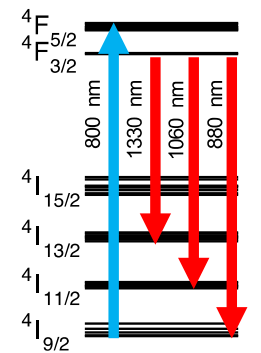

(b) Neodymium $\left(\mathrm{Nd}^{3+}\right)$

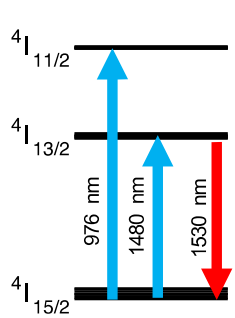

(c) Erbium $\left(\mathrm{Er}^{3+}\right)$

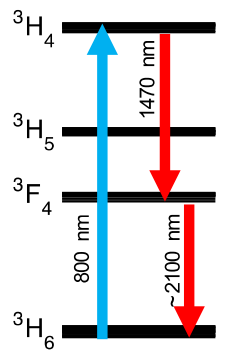

(d) Thulium $\left(\mathrm{Tm}^{3+}\right)$

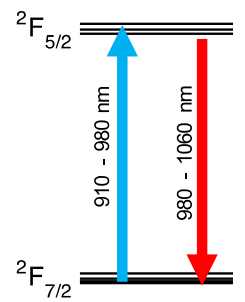

(e) Ytterbium $\left(\mathrm{Yb}^{3+}\right)$

Figure 1.1 Simplified energy level diagrams of trivalent (a) praseodymium, (b) neodymium, (c) erbium, (d) thulium, and (e) ytterbium. The arrows indicate commonly used pump and signal transitions. The actual transition wavelengths are depending on host material and operating condition. Higher energy levels of praseodymium, neodymium, erbium, and thulium within the $4 f$ shell are not shown in the figure. 
The transition of erbium ion $\left(\mathrm{Er}^{3+}\right)$ at $\sim 1530 \mathrm{~nm}$ wavelength coincides with low optical attenuation wavelength range in silica fiber, hence it is widely used for telecommunication purpose. Materials doped with trivalent neodymium $\left(\mathrm{Nd}^{3+}\right)$ produces luminescence at about $880 \mathrm{~nm}, 1060 \mathrm{~nm}$, and $1330 \mathrm{~nm}$ [22]. The emission wavelengths at $\sim 880 \mathrm{~nm}$ and $\sim 1330 \mathrm{~nm}$ are near to the first and second fiber communication windows, respectively. However, amplification at these wavelengths is challenging as the strongest luminescence occurs at $1060 \mathrm{~nm}$. The gain spectrum of praseodymium-doped material has a peak at $\sim 1300 \mathrm{~nm}$ wavelength and covers the wavelength range of 1290-1330 $\mathrm{nm}$ [23], therefore praseodymium-doped material is a more favorable candidate than $\mathrm{Nd}^{3+}$-doped material for amplification at the second fiber communication window. $\mathrm{Yb}^{3+}$ possesses simple electronic level structure with only an excited state and a ground state. Both $\mathrm{Yb}^{3+}$ and $\mathrm{Nd}^{3+}$ emit at $\sim 1000 \mathrm{~nm}$ wavelength, hence they are suitable for novel short-reach interconnects used in large scale computing systems $[6,24]$. The emission of thulium at $\sim 1470 \mathrm{~nm}$ is useful for signal amplification in the S-band (1460-1530 nm) [25]. Amplification at $\sim 2 \mu \mathrm{m}$ wavelength using thulium-doped fiber [26] has also received considerable attention lately as this wavelength region may be used to further extend the fiber communication window.

Consider a simplified two energy levels system for the rare-earth-ion-doped material, for instance $\mathrm{Yb}^{3+}$-doped material, the optical gain achievable in the material is governed by the gain coefficient,

$$
g=\sigma_{e m} N_{1}-\sigma_{a b s} N_{0} .
$$

The effective emission cross-section $\sigma_{e m}$ and the effective absorption cross-section $\sigma_{a b s}$ represent the probability of the material in emitting and absorbing photons at certain wavelength. $N_{1}$ and $N_{0}$ represent the density of active ion at the excited state and ground state, respectively, and the sum of $N_{1}$ and $N_{0}$ is the total density of active ions in the medium $N_{d}$. Therefore, the upper limit of the gain in rare-earth-ion-doped material can be extended by using host material which provides high transition cross-sections or by incorporating higher density of active ions in the material.

The transition linewidth in rare-earth-ion-doped material is affected by homogeneous and inhomogeneous broadening mechanisms. Homogeneous broadening is applicable to both crystalline and amorphous materials and it is caused by lifetime broadening which is dominated by rapid phonon-induced transitions between the Stark components within a given multiplet [23]. Inhomogeneous broadening, on the other hand, originates from local site-to-site variation of the surrounding crystal field which results in a distribution of energies for a given Stark component [23]. Rare-earth-iondoped amorphous host materials, such as silica glass, phosphate glass, and aluminum oxide, exhibit both homogeneous and inhomogeneous broadening mechanisms. Therefore, the absorption and emission spectra in these materials are smoother and 
broader than the rare-earth-ion-doped crystalline materials. Consequently, the transition cross-sections in crystalline hosts are usually higher than amorphous materials.

The increase of active ion concentration in the medium could lead to concentration quenching effect which causes shortening of excited state lifetime. The reduction of lifetime with higher active ion concentration can arise from the increased probability of energy transfer towards impurities, voids, defects, or other quenching centers [27]. The amount of active ions in certain amorphous materials such as glass fiber is limited to a rather low level to prevent the formation of microscopic clusters. This is because the clustering of active ions may lead to undesirable energy transfer between the ions, hence degrading the gain and power efficiency. Apart from that, the increase of active ion concentration could increase the magnitude of interionic processes such as energy transfer upconversion and cross-relaxation, which in turn impose a limit to the gain achievable in the material $[22,28]$.

\subsection{State-of-the-art waveguide amplifiers}

Waveguide amplifiers can be categorized into semiconductor optical amplifier (SOA) and rare-earth-ion-doped waveguide amplifier. The SOA can be pumped electrically as compared to the rare-earth-ion-doped waveguide amplifier which requires optical pumping. Besides, SOA possesses modal gain exceeding $1000 \mathrm{~dB} / \mathrm{cm}[9,21]$, which is significantly higher than most of the rare-earth-ion-doped materials.

The rare-earth-ion-doped waveguide amplifier is a better candidate for applications requiring high data rate amplification, such as short range optical interconnects which are currently operating at 10-25 Gbps [6, 7, 29] and progressing towards $\geq 40$ Gbps [30-33]. Thanks to the long excited state lifetime up to $7.5 \mathrm{~ms}$ [28], high bit rate amplification at $170 \mathrm{Gbps}$ had been demonstrated in erbium-doped waveguide amplifier without significant gain modulation [34]. On the other hand, high bit rate signal amplification (e.g. $\geq 10 \mathrm{Gbps}$ ) using SOA working in saturation region may lead to intersymbol interference because the short carrier lifetime in SOA (in the order of $\sim 100 \mathrm{ps}$ ) is comparable to the signal's bit rate [19].

Apart from that, rare-earth-ion-doped amplifier generally exhibits higher gain bandwidth and better noise figure than SOA. High gain bandwidth is desirable to accommodate more signal channels in a wavelength multiplexed system. The noise figure represents the degradation of the signal-to-noise ratio of the signal beam after passing through the amplifier. Till date, gain bandwidth of 55-80 $\mathrm{nm}[35,36]$ and noise figure of $3.75 \mathrm{~dB}$ [37] had been demonstrated in rare-earth-ion-doped waveguide amplifiers, while typical gain bandwidth and noise figure for the SOAs are $\sim 30-50 \mathrm{~nm}$ and 5-12 $\mathrm{dB}[38,39]$, respectively. Moreover, active cooling is essential for SOA 
especially when it is bonded onto passive waveguide $[9,40]$ whereas $15-20 \mathrm{~dB}$ of net gain can be achieved in rare-earth-ion-doped waveguide amplifiers without any thermal management measure [20, 22, 37, 41].

The mainstream of the development on rare-earth-ion-doped waveguide amplifiers is concentrating on $\mathrm{Er}^{3+}$-doped material. Nonetheless, a number of high performance waveguide amplifiers have also been reported on $\mathrm{Nd}^{3+}$ - and $\mathrm{Yb}^{3+}$-doped materials. Figure 1.2 displays an overview of the net gain per unit length $(\mathrm{dB} / \mathrm{cm})$ versus net gain $(\mathrm{dB})$ data for various types of waveguide amplifiers reported since 1990. A higher net gain per unit length is desirable for amplifiers as it infers that shorter device length can be used to achieve a given amount of total gain. However, this figure of merit alone does not provide a complete picture because the actual total gain realized could be rather low. Therefore, the knowledge on the total net gain achieved is also important. Two reference points for erbium-doped fiber amplifiers (EDFA) $[42,43]$ are included in Figure 1.2 (red squares at the bottom right corner) for benchmarking purpose. Efficient operation is typically obtained in conventional EDFA with $\sim 10^{18}$ $\mathrm{Er}^{3+}$ ions $/ \mathrm{cm}^{3}[42,43]$ as further increasing the $\mathrm{Er}^{3+}$ concentration would lead to microscopic clustering and undesirable ion-ion interactions in the material [23]. Therefore, fiber lengths longer than a few meters are needed to deliver net gain $>20 \mathrm{~dB}$, resulting in low net gain per unit length figures for the EDFAs.

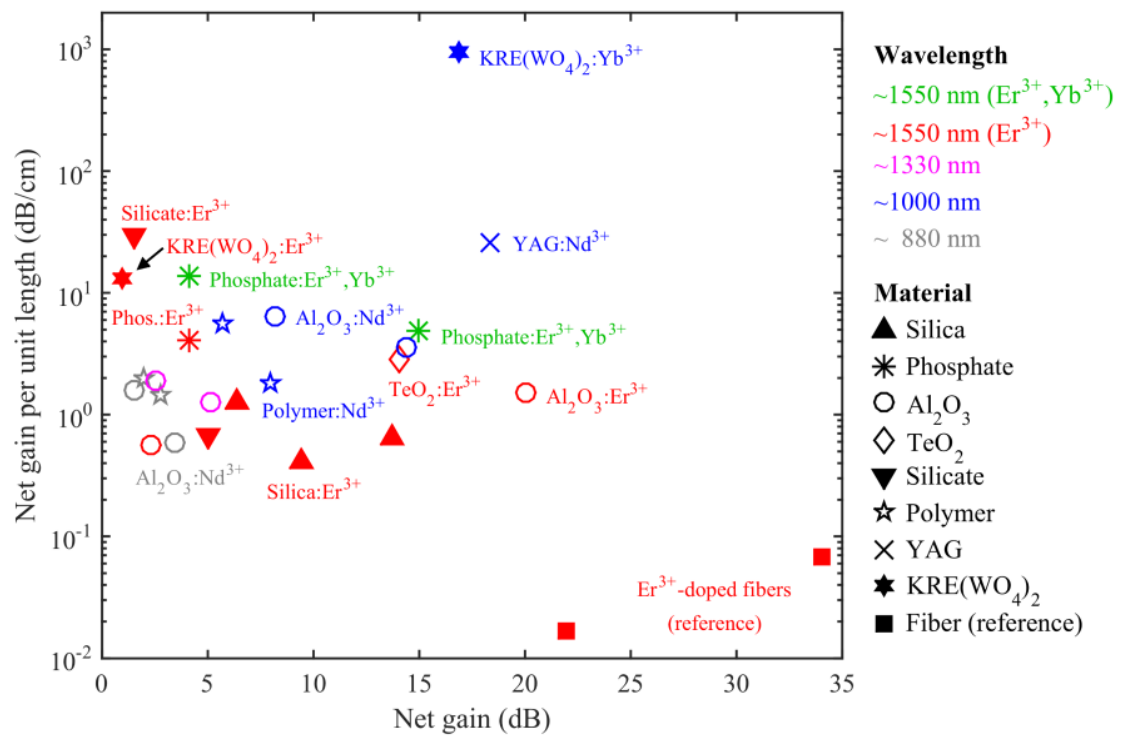

Figure 1.2 Comparison of key internal net gain (dB) and internal net gain per unit length $(\mathrm{dB} / \mathrm{cm})$ results obtained from various types of waveguide amplifiers. Markers used to represent the host materials are: triangle (silica), asterisk (phosphate), circle $\left(\mathrm{Al}_{2} \mathrm{O}_{3}\right)$, diamond (tellurium dioxide), inverted triangle (silicate), pentagon (polymer), cross (YAG), hexagon [KRE( $\left(\mathrm{WO}_{4}\right)_{2}$ ], and square (fiber). The color represents the operating wavelength region of the given waveguide amplifiers. The data points and the corresponding references can be found in Appendix. 
The development of rare-earth-ion-doped waveguide amplifiers was started on silica glasses. $\mathrm{Er}^{3+}$-doped waveguide amplifiers (EDWA) based on silica (red triangles in Figure 1.2) with net gain as high as $13.7 \mathrm{~dB}$ had been reported in early 90s [44]. However, limited refractive index contrast in silica glass leads to large optical mode. As a result, high pump power is required to achieve net gain. The net gain per unit length of EDWA remained low $(<0.7 \mathrm{~dB} / \mathrm{cm})$ [45-47] until the introduction of multicomponent phosphate glass waveguide amplifier with net gain per unit length of $4.1 \mathrm{~dB} / \mathrm{cm}$ (red asterisk) [48]. EDWA fabricated from $\mathrm{Al}_{2} \mathrm{O}_{3}$ (red circles) was reported in 1996 [47]. Later development had led to $12.9 \mathrm{~cm}$ long $\mathrm{Al}_{2} \mathrm{O}_{3}: \mathrm{Er}^{3+}$ waveguide amplifiers with record high $20 \mathrm{~dB}$ net gain [37]. In addition, high bit-rate amplification at $170 \mathrm{~Gb} / \mathrm{s}$ [34], gain bandwidth over $80 \mathrm{~nm}$ [35] in the C-band, and peak gain per unit length of $\sim 2.0 \mathrm{~dB} / \mathrm{cm}$ [35] had been reported in $\mathrm{Al}_{2} \mathrm{O}_{3}: \mathrm{Er}^{3+}$. Monolithic integration of $\mathrm{Al}_{2} \mathrm{O}_{3}: \mathrm{Er}^{3+}$ amplifier on silicon-on-insulator platform had also been demonstrated [13]. Apart from that, EDWA based on tellurium dioxide $\left(\mathrm{TeO}_{2}\right)$ with $2.8 \mathrm{~dB} / \mathrm{cm}$ net gain per unit length (see red diamond in Figure 1.2) had been demonstrated [49]. Net gain of $14 \mathrm{~dB}$ was achieved using $5 \mathrm{~cm}$ long $\mathrm{TeO}_{2}: \mathrm{Er}^{3+}$ waveguides by pumping at $1480 \mathrm{~nm}$ [49]. Similar gain figure was also obtained by pumping at $980 \mathrm{~nm}$ wavelength, but the gain achievable in $\mathrm{TeO}_{2}: \mathrm{Er}^{3+}$ could be higher since the gain measurement at higher pump power was limited by the onset of lasing [50]. Recently, crystalline $\mathrm{Er}^{3+}$-doped potassium rare-earth double tungstates, $\mathrm{KRE}\left(\mathrm{WO}_{4}\right)_{2}: \mathrm{Er}^{3+}$ waveguide with net gain per unit length of $13 \mathrm{~dB} / \mathrm{cm}$ had been reported (red hexagon). Photonics crystal slot waveguide based on silicates $\left(\mathrm{Er}_{0.4} \mathrm{Y}_{1.6} \mathrm{SiO}_{5}\right)$ exhibiting net gain per unit length as high as $30 \mathrm{~dB} / \mathrm{cm}$ (red inverted triangle) was also reported [51].

Co-doping with $\mathrm{Yb}^{3+}$ effectively enhances the pump absorption in EDWAs as $\mathrm{Yb}^{3+}$ generally exhibits high absorption band at $\sim 980 \mathrm{~nm}$. The transfer of energy from $\mathrm{Yb}^{3+}$ to $\mathrm{Er}^{3+}$ allows similar magnitude of pump absorption over a waveguide length shorter than those of singly $\mathrm{Er}^{3+}$-doped waveguides. The EDWAs co-doped with $\mathrm{Yb}^{3+}$ are shown in green color in Figure 1.2 in order to distinguish them from the singly $\mathrm{Er}^{3+}$ doped amplifiers which are shown in red. In the case of phosphate glass, co-doping with $\mathrm{Yb}^{3+}$ significantly raises the net gain to $15 \mathrm{~dB}$ [41] and net gain per unit length to $13.67 \mathrm{~dB} / \mathrm{cm}$ [52] (green asterisks). Nevertheless, non-unity energy transfer efficiency from $\mathrm{Yb}^{3+}$ ions to $\mathrm{Er}^{3+}$ ions [53], fraction of $\mathrm{Yb}^{3+}$ ions not contributing to energy exchange with $\mathrm{Er}^{3+}$ ions [54], as well as waste of pump energy due to cumulativetransfer process which leads to further excitation of $\mathrm{Er}^{3+}$ ions to upper energy levels [53, 54] will result in lower gain in $\mathrm{Er}^{3+}-\mathrm{Yb}^{3+}$ co-doped amplifiers as compared to singly $\mathrm{Er}^{3+}$-doped amplifiers given the same amount of absorbed pump power.

Waveguide amplifiers operating at other wavelength regions had also been developed. For instance, waveguide amplifiers made of $\mathrm{Nd}^{3+}$-doped $\mathrm{Al}_{2} \mathrm{O}_{3}$ [22] and $\mathrm{Nd}^{3+}$-doped polymer (pentagons in Figure 1.2) [55-57] can operate at $\sim 880 \mathrm{~nm}$, 
$1064 \mathrm{~nm}$, and $1330 \mathrm{~nm}$ wavelengths. Integration of these amplifiers with optical backplane at $\sim 880 \mathrm{~nm}$ operating wavelength was demonstrated [57]. The reported best gain figures for $\mathrm{Nd}^{3+}$-doped $\mathrm{Al}_{2} \mathrm{O}_{3}$ waveguides are $14.4 \mathrm{~dB}$ and $6.3 \mathrm{~dB} / \mathrm{cm}$ at $1064 \mathrm{~nm}$ wavelength [22] (blue pentagons), while they are $8 \mathrm{~dB}$ and $5.7 \mathrm{~dB} / \mathrm{cm}$ for $\mathrm{Nd}^{3+}$-doped polymer waveguides [56] (blue circle). A number of remarkable gain per unit length results had been reported at $\sim 1000 \mathrm{~nm}$ wavelength region lately. Gain per unit length of $26.3 \mathrm{~dB} / \mathrm{cm}$ was reported on ceramic YAG: $\mathrm{Nd}^{3+}$ waveguide amplifier (blue cross) [58]. Besides, based on the spectroscopic findings, a theoretical gain of $78 \mathrm{~dB} / \mathrm{cm}$ was anticipated on $\mathrm{Yb}^{3+}$-doped tantalum pentoxide [59] (not shown). A breakthrough gain figure of $935 \mathrm{~dB} / \mathrm{cm}$ was measured from $\mathrm{Yb}^{3+}$-doped potassium rare-earth double tungstates, $\mathrm{KRE}\left(\mathrm{WO}_{4}\right)_{2}: \mathrm{Yb}^{3+}$, at $981 \mathrm{~nm}$ wavelength [20] (blue hexagon), which is about two orders of magnitude higher than most of the singly-doped waveguide amplifiers. The $\mathrm{KRE}\left(\mathrm{WO}_{4}\right)_{2}: \mathrm{Yb}^{3+}$ amplifier which was as short as $180 \mu \mathrm{m}$ produced a high total net gain of $16.83 \mathrm{~dB}$.

\subsection{Ytterbium-activated \\ potassium double tungstate amplifiers}

The research work presented in this thesis is based on crystalline potassium rare-earth double tungstate waveguide layers activated with $\mathrm{Yb}^{3+}$, i.e. $\mathrm{KRE}\left(\mathrm{WO}_{4}\right)_{2}: \mathrm{Yb}^{3+}$. These layers are grown on undoped potassium double tungstate substrate using cost-effective liquid phase epitaxy technique [60-63]. By co-doping the $\mathrm{Yb}^{3+}$-activated layer with optically inert trivalent gadolinium $\left(\mathrm{Gd}^{3+}\right)$ and/or lutetium $\left(\mathrm{Lu}^{3+}\right)$, the refractive index contrast of the layer with respect to the substrate can be enhanced for waveguiding purpose [64, 65]. Epitaxial layers grown using this technique had been used to demonstrate continuous wave $(\mathrm{CW})$ planar waveguide laser with slope efficiency as high as $82.3 \%$ [66] as well as channel waveguide laser with $71 \%$ slope efficiency and high CW output power of $418 \mathrm{~mW}$ [67]. Besides, the growth technique had been applied to grow thulium-doped epitaxial layers for the realization of channel waveguide laser emitting at $2 \mu \mathrm{m}$ wavelength with record high slope efficiency of $\sim 80 \%$ and $1.6 \mathrm{~W}$ output power [68].

The epitaxial layer growth technique and waveguide structuring technique had also been used to realize high gain $\mathrm{Yb}^{3+}$-activated [20] and $\mathrm{Er}^{3+}$-activated [69] waveguide amplifiers. The $\mathrm{KRE}\left(\mathrm{WO}_{4}\right)_{2}: \mathrm{Yb}^{3+}$ amplifier with $935 \mathrm{~dB} / \mathrm{cm}$ net gain per unit length [20] mentioned in previous section consists of $\mathrm{KGd}_{0.447} \mathrm{Lu}_{0.078} \mathrm{Yb}_{0.475}\left(\mathrm{WO}_{4}\right)_{2}$ layer grown on undoped potassium yttrium double tungstate, i.e. $\mathrm{KY}\left(\mathrm{WO}_{4}\right)_{2}$, substrate. The 47.5 at. $\% \mathrm{Yb}^{3+}$ in the layer corresponds to an active ion density of $3 \times 10^{21} \mathrm{~cm}^{-3}$. The outstandingly high gain per unit length of $\mathrm{KRE}\left(\mathrm{WO}_{4}\right)_{2}: \mathrm{Yb}^{3+}$ waveguide amplifier is 
a result of thorough consideration on multiple important aspects. Firstly, active media doped with $\mathrm{Yb}^{3+}$ exhibit a simple energy-level scheme consisting of only ${ }^{2} \mathrm{~F}_{5 / 2}$ excited state and ${ }^{2} \mathrm{~F}_{7 / 2}$ ground state. Consequently, parasitic processes, such as energy-transfer upconversion, cross-relaxation, and excited-state absorption, which limit the population inversion are absent in principle. Secondly, the material gain of $\mathrm{KRE}\left(\mathrm{WO}_{4}\right)_{2}: \mathrm{Yb}^{3+}$ is inherently higher due to its high transition cross-sections which are superior to many other $\mathrm{Yb}^{3+}$-doped materials [59, 70-74]. Thirdly, the use of co-doped $\mathrm{Gd}^{3+}$ and $\mathrm{Lu}^{3+}$ in the layer allows the growth of high $\mathrm{Yb}^{3+}$ concentration layer with refractive index contrast of $\sim 0.01-0.02$ with respect to the substrate [65]. Furthermore, with appropriate micro-structuring of the highly $\mathrm{Yb}^{3+}$-doped $\mathrm{KRE}\left(\mathrm{WO}_{4}\right)_{2}$ layer, the resulting channel waveguide allow for high optical intensity to be maintained over a rather long interaction length, hence greatly enhances the light-matter interaction within a limited physical device length. Following the successful work shown in [20], it is worthwhile to examine if the gain could be further extended by using epitaxial layer with higher $\mathrm{Yb}^{3+}$ concentration.

In this thesis, the use of $\operatorname{KRE}\left(\mathrm{WO}_{4}\right)_{2}$ epitaxial layers with more than 50 at.\% $\mathrm{Yb}^{3+}$ concentration for optical amplification purpose is investigated. The attention of this work is put on the operation scheme with $\sim 932 \mathrm{~nm}$ pump wavelength and $\sim 981 \mathrm{~nm}$ signal wavelength. Figure 1.3 shows the representative absorption cross-section and emission cross-section spectra in $\mathrm{KRE}\left(\mathrm{WO}_{4}\right)_{2}: \mathrm{Yb}^{3+}$. The broad absorption band at $\sim 932 \mathrm{~nm}$ (see blue curve in Figure 1.3) allows the eventual device to be pumped using high wall-plug efficiency InGaAs lasers [75]. In the absent of optical pumping, the material is highly absorbing at $\sim 981 \mathrm{~nm}$ wavelength. The application of optical pump promotes the $\mathrm{Yb}^{3+}$ ions to excited state, hence reduces the absorption accordingly. The increase of pump power will lead to higher $\mathrm{Yb}^{3+}$ population at the excited state. Under intense pumping condition, a maximum population inversion can be achieved and gain cross-section spectrum represented by the black dotted curve in Figure 1.3 is obtained. Therefore, by intensely pumping at $\sim 932 \mathrm{~nm}$ wavelength, a peak gain can be obtained at $\sim 981 \mathrm{~nm}$ and a broadband gain bandwidth of $55 \mathrm{~nm}$ is attainable within the wavelength range of 977-1032 nm [36]. The high gain at the central line near $981 \mathrm{~nm}$ may be exploited for potential signal amplification in short reach optical interconnects operating near $980 \mathrm{~nm}$ [6] or in any other application where amplification is desired. 


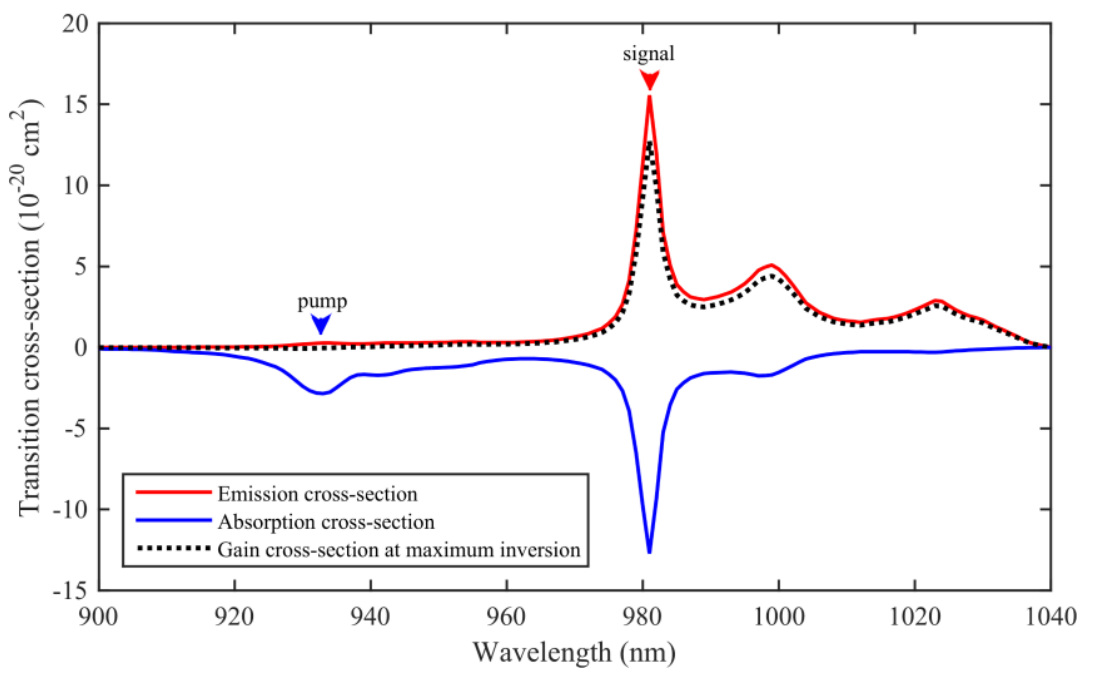

Figure 1.3 Absorption and emission cross-section spectra in bulk crystal of $\mathrm{KY}\left(\mathrm{WO}_{4}\right)_{2}: \mathrm{Yb}^{3+}$. The arrows indicate the operation wavelengths of interest in this work. The black dotted curve shows the calculated gain cross-section under theoretical maximum population inversion condition (Eqs. 5.34 and 5.35). The absorption cross-section spectrum is obtained from the work of Kuleshov et al. [71] while the emission cross-section is calculated using reciprocity method. Further information about the calculation can be found in Sections 2.3.4 and 4.3.4.

\subsection{Outline of this thesis}

This thesis focuses on the experimental and theoretical investigations on optical properties of high $\mathrm{Yb}^{3+}$ concentration waveguide layers which are catered for optical amplification purpose.

In the subsequent chapter, relevant theories necessary for the understanding of the ytterbium-activated waveguide amplifiers will be outlined. The chapter begins with a brief introduction of the properties of rare-earth elements. Subsequently, the basis of the spectroscopy and optical gain/loss will be discussed. A brief summary on the reported optical waveguides based on potassium double tungstates is also given in this chapter.

Chapter 3 covers an analysis of the material properties of bulk potassium double tungstate crystals and the details about the preparation of epitaxial layers used in this thesis. Two methods used to grow high $\mathrm{Yb}^{3+}$ concentration epitaxial layers for optical amplification purpose are explained. This chapter also includes the information about the liquid phase epitaxy growth procedure as well as several processing and characterization methods which are applicable to most of the samples.

The spectroscopic properties of the high $\mathrm{Yb}^{3+}$ concentration epitaxial layers are reported in Chapter 4. These include the results of luminescence lifetime measurement on samples with various $\mathrm{Yb}^{3+}$ concentrations. The transition cross-sections of the 


\section{Chapter 1}

epitaxial layers with 57 at.\% and 76 at.\% $\mathrm{Yb}^{3+}$ are reported. Moreover, the temperature dependence of these spectroscopic properties are investigated in this chapter.

Next, the pump absorption and signal gain in epitaxial layer with 57 at.\% $\mathrm{Yb}^{3+}$ are examined in Chapter 5. A numerical model which takes into account the pump-induced heating at the focused spot is established. The experimental and numerical results are discussed and analyzed.

Finally, the conclusions on the work performed in this thesis can be found in Chapter 6. An outlook of the future work will be presented before the end of this thesis. 


\section{Chapter 2}

\section{Theoretical background}

\subsection{Overview}

This chapter introduces the fundamentals of ytterbium-doped waveguide amplifiers. Ytterbium belongs to the rare-earth elements which share some common characteristics. The light and matter interaction processes in rare-earth activated material are elaborated in a general context. Important optical transition parameters are defined and explained to provide a foundation for the spectroscopic and optical gain experiments in later chapters. Apart from that, the methods used to realize optical waveguides on potassium double tungstates will be discussed.

\subsection{Rare-earth elements}

Ytterbium $(\mathrm{Yb})$ is grouped in the periodic table as one of the lanthanides, which include elements with atomic numbers ranging from $Z=57$ (lanthanum) to $Z=71$ (lutetium). Scandium (Sc) and yttrium (Y) exhibit chemical properties similar to the lanthanides and they tend to reside in the same ore, hence they are often associated with the rareearth elements. These elements are indicated in the periodic table shown in Figure 2.1.

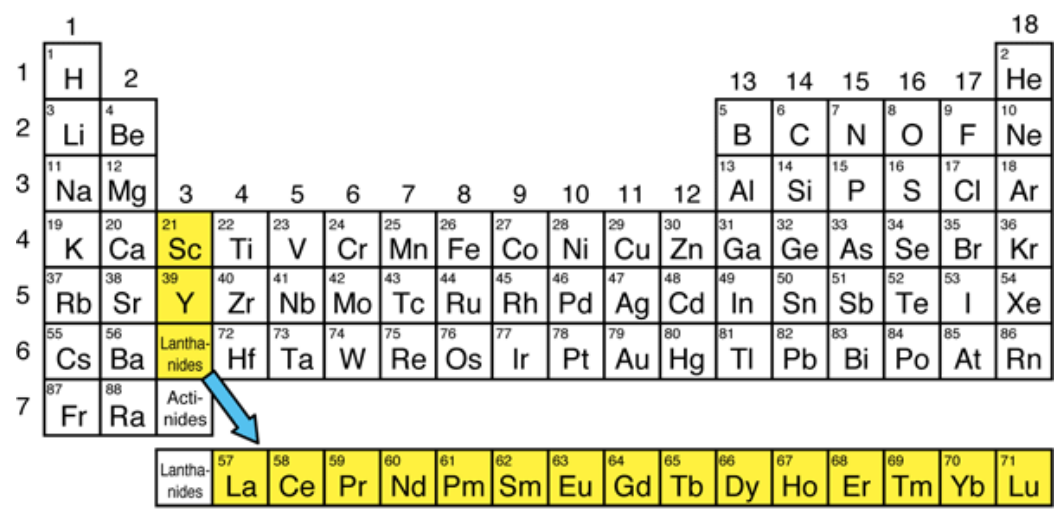

Figure 2.1 Periodic table indicating the rare-earth elements [76]. The elements particularly relevant to this work are yttrium (Y), gadolinium (Gd), ytterbium (Yb), and lutetium (Lu). 
The lanthanides are associated with the filling of $4 f$ shell. Since the $4 f$ shell of the lanthanides is partially shielded by the outer shells, the $4 f$ energy levels and the transitions within the $4 f$ shell for these elements are only slightly different from one host to the other. The neutral lanthanides have common electronic configuration $[\mathrm{Xe}] 4 f^{n} 5 d^{m} 6 s^{2}$, where [Xe] represents a Xenon core. With the increasing atomic number, the number of electrons in the $4 f$ shell increases from lanthanum $(n=0)$ to lutetium $(n=14)$, which exhibits an electronic configuration $[\mathrm{Xe}] 4 f^{14} 5 d^{1} 6 s^{2}$. The $5 d$ shell for lanthanum (La), cerium (Ce), gadolinium (Gd), and lutetium (Lu) is characterized by 1 electron $(m=1)$ whereas the other lanthanides have no electron in their $5 d$ shell $(m=0)$. When the lanthanides are included in a host, they typically exist in trivalent charge state, giving up two loosely bound $6 s$ electrons and one electron from either $5 d$ or $4 f$ shell. Consequently, $\mathrm{La}^{3+}$ and $\mathrm{Lu}^{3+}$ ions do not interact with optical radiation as their $4 f$ shell is either empty or completely filled.

The trivalent ytterbium ion $\left(\mathrm{Yb}^{3+}\right)$ with 13 electrons in its $4 f$ shell has a simple energy level scheme. The energy levels can be represented by Russell-Saunders notation ${ }^{2 \mathrm{~S}+1} \mathrm{~L}_{\mathrm{J}}$, where $\mathrm{S}$ is the total spin, $\mathrm{L}$ is the total orbital angular momentum, and $\mathrm{J}$ is the total angular momentum [23]. The spin-orbit coupling in $\mathrm{Yb}^{3+}$ produces only two manifolds, i.e. ${ }^{2} \mathrm{~F}_{5 / 2}$ upper state and the ${ }^{2} \mathrm{~F}_{7 / 2}$ ground state manifolds, which are separated by $\sim 10000 \mathrm{~cm}^{-1}$. These manifolds contain three and four Starks levels, respectively. Due to its simple energy level scheme, parasitic processes which are usually detrimental for amplification and lasing, such as energy-transfer upconversion, cross-relaxation, and excited-state absorption, are in principle absent in $\mathrm{Yb}^{3+}$-activated media. Unfortunately, traces of impurities such as $\mathrm{Nd}^{3+}, \mathrm{Er}^{3+}$, or $\mathrm{Tm}^{3+}$ are often found in $\mathrm{Yb}^{3+}$-activated media [77]. The presence of these ions may introduce undesirable energy transfer processes which deplete the excited population from the ${ }^{2} \mathrm{~F}_{5 / 2}$ upper state in $\mathrm{Yb}^{3+}$, resulting in deteriorated device performance. Besides, cooperative upconversion from pairs of $\mathrm{Yb}^{3+}$ ions [78], in which the de-excitation of two $\mathrm{Yb}^{3+}$ ions produces emission of photon in visible wavelength, could also occur.

Apart from $\mathrm{Yb}^{3+}$, the present work also involves other trivalent rare-earth ions such as gadolinium $\left(\mathrm{Gd}^{3+}\right)$, lutetium $\left(\mathrm{Lu}^{3+}\right)$, and yttrium $\left(\mathrm{Y}^{3+}\right)$. As the first upper state in $\mathrm{Gd}^{3+}$ is $\sim 36000 \mathrm{~cm}^{-1}$ from the ground state [79], direct energy transfer process between the energy levels in $\mathrm{Yb}^{3+}$ and the energy levels in $\mathrm{Gd}^{3+}$ is not probable. $\mathrm{Lu}^{3+}$ is not optically active as its $4 f$ shell is completely filled. Yttrium has been traditionally used as the host for $\mathrm{Yb}^{3+}$-doped crystals, such as Yttria $\left(\mathrm{Y}_{2} \mathrm{O}_{3}\right)$, YAG (yttrium aluminum garnet $\left.-\mathrm{Y}_{3} \mathrm{Al}_{5} \mathrm{O}_{12}\right)$, and $\mathrm{YAP}\left(\mathrm{YAlO}_{3}\right)$ as it does not interact with the radiations at the working wavelengths of $\mathrm{Yb}^{3+}$. Therefore, $\mathrm{Gd}^{3+}, \mathrm{Lu}^{3+}$ and $\mathrm{Y}^{3+}$ are considered as optically inert codopant in $\mathrm{Yb}^{3+}$-activated media. This serves as the basis of the lattice engineering approach used to fabricate high $\mathrm{Yb}^{3+}$ concentration epitaxial layer in this work, which will be further elaborated in the subsequent chapter. 


\subsection{Interaction of light and matter}

The fundamental interactions between a radiation field and an atomic system can be categorized into stimulated absorption, spontaneous emission and stimulated emission [80]. These interactions, which are defined using the Einstein coefficients, are discussed in this section using a simplified case involving only two atomic energy levels. Nevertheless, the energy level scheme of the rare-earth ions is more complex than such simple two-level scheme. Even for the case of $\mathrm{Yb}^{3+}$, which exhibits only a ${ }^{2} \mathrm{~F}_{5 / 2}$ upper state and a ${ }^{2} \mathrm{~F}_{7 / 2}$ ground state manifolds, the existence of Stark levels leads to a statistical distribution of total populations within the manifolds. These sub-levels also allow a combination of transitions which may spectrally overlap with each other. The relevant spectroscopic parameters which take into account the Stark split levels are introduced. In addition, typical theories used for spectroscopic study on rare-earth ions, particularly in the context of host materials doped with $\mathrm{Yb}^{3+}$, will be reviewed.

\subsubsection{Absorption, spontaneous emission, and stimulated emission}

Consider an atomic system having only a lower energy level $E_{0}$ and a higher energy level $E_{1}$ as shown in Figure 2.2. The difference between the energy levels yields $h v$, where $h$ is Plank's constant and $v$ is the frequency of the radiation. The atoms per unit volume residing on these energy levels are denoted by $N_{0}$ and $N_{1}$, respectively, which can be determined based on the Boltzmann distribution [81]

$$
\frac{N_{1}}{N_{0}}=\frac{g_{1}}{g_{0}} \exp \left(-\frac{E_{1}-E_{0}}{k_{B} T}\right),
$$

where $g_{\mathrm{i}}$ is degeneracy parameter of the energy level, $k_{B}$ is Boltzmann constant, and $T$ is temperature. Therefore, given an energy gap much larger than the thermal energy, i.e. $E_{1}-E_{0} \gg k_{B} T$, most of the atoms will stay at the ground state with energy $E_{0}$.

(a) Absorption

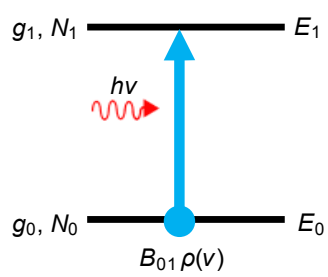

(b) Spontaneous emission

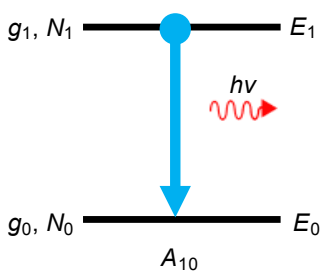

(c) Stimulated emission

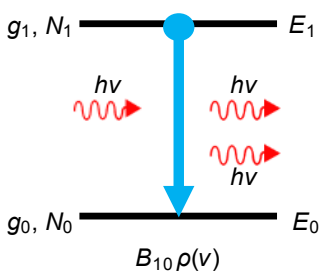

Figure 2.2 Schematic of the light and matter interaction processes in a two-level system: (a) absorption, (b) spontaneous emission, and (c) stimulated emission. 


\section{Absorption}

An atom residing at the ground state may absorb a photon with energy $h v$. As a result, the atom is promoted to the excited state with energy $E_{1}$ as indicated in Figure 2.2(a). The interaction process can be expressed by

$$
\frac{d N_{0}}{d t}=-B_{01} \rho(v) N_{0},
$$

where $B_{01}$ represents the coefficient of stimulated absorption and $\rho(v)$ is the spectral energy density of the radiation per unit frequency $v$.

\section{Spontaneous emission}

An atom previously promoted to the excited state may relax spontaneously to the ground state and emits a photon without excitation from external causes, as depicted in Figure 2.2(b). Such emission is known as spontaneous emission. The probability per unit time that the atoms decay spontaneously is defined by

$$
\frac{d N_{1}}{d t}=-A_{10} N_{1}
$$

where $A_{10}$ represents the coefficient of spontaneous decay.

\section{Stimulated emission}

Stimulated emission occurs when the atom at the excited state are irradiated with photon of energy $h v$. The impinging photon stimulates a relaxation of atom from the excited state to the ground state as illustrated in Figure 2.2(c). The relaxation of energy produces another photon with frequency, polarization, and direction of propagation identical to the photon stimulating the process. The process of stimulated emission can be described with

$$
\frac{d N_{1}}{d t}=-B_{10} \rho(v) N_{1}
$$

where $B_{10}$ is the coefficient of stimulated emission.

\section{Relationship between the Einstein coefficients}

The $B_{01}, A_{10}$, and $B_{10}$ are collectively known as the Einstein coefficients. Under equilibrium condition, there is no net change in the density of atoms at both $E_{0}$ and $E_{1}$ energy levels. Consequently, the absorption process which promotes the atoms to the $E_{1}$ level is balanced by the spontaneous and stimulated emission processes which deplete the atoms from the same level,

$$
B_{01} \rho(v) N_{0}=A_{10} N_{1}+B_{10} \rho(v) N_{1} .
$$


Substituting Eq. 2.1 into Eq. 2.5 yields

$$
\rho(v)=\frac{A_{10}}{B_{01} \frac{g_{0}}{g_{1}} \exp \left(h v / k_{B} T\right)-B_{10}} .
$$

By comparing the above expression to Plank's law

$$
\rho(v)=\frac{8 \pi h v^{3}}{c^{3}} \frac{1}{\exp \left(h v / k_{B} T\right)-B_{21}},
$$

the relationship between the Einstein coefficients $B_{01}, A_{10}$ and $B_{10}$ can be determined

$$
\frac{A_{10}}{B_{10}}=\frac{8 \pi h v^{3}}{c^{3}},
$$

and

$$
\frac{B_{10}}{B_{01}}=\frac{g_{0}}{g_{1}} .
$$

Equations 2.8 and 2.9 show that the absorption, the spontaneous emission, and the stimulated emission are closely related to each other. Given a steady-state system, knowing one of the Einstein coefficients allows the calculation of the remaining two coefficients.

\subsubsection{Lifetime and effective cross-sections}

As the manifolds of rare-earth ions contain Stark split levels, an extension of the simple two-level scheme is needed to represent the spectroscopic properties observed in the measurements. Figure 2.3 shows the extended energy level scheme taking into account the Stark splitting, where the lower state with energy $E_{0}$ is split into $m$-levels and the upper state with energy $E_{1}$ is split into $n$-levels. Due to the Stark splitting, a combination of $m \times n$ transitions may occur. Besides, the total population within each manifold will be distributed among the Stark levels. Assuming that rapid thermalisation takes place within the manifold, the fractional population at the $i$-th Stark level within the lower state can be deduced with the Boltzmann distribution

$$
f_{0 i}(T)=\frac{\exp \left[-\left(E_{0 i}-E_{00}\right) / k_{B} T\right]}{\sum_{p=0}^{m} \exp \left[-\left(E_{0 p}-E_{00}\right) / k_{B} T\right]} .
$$




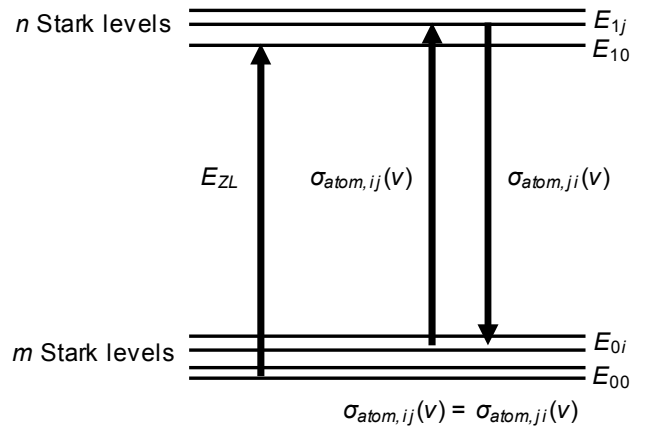

Figure 2.3 Diagram illustrating the general case of two manifolds with a number of Stark levels. The $\mathrm{Yb}^{3+}$ possesses three Stark levels at the ${ }^{2} \mathbf{F}_{5 / 2}$ upper state $(n=3)$ and four Stark levels at the ${ }^{2} F_{7 / 2}$ ground state $(m=4)$.

Similarly, the fractional population at the $j$-th Stark level within the upper state can be calculated using

$$
f_{1 j}(T)=\frac{\exp \left[-\left(E_{1 j}-E_{10}\right) / k_{B} T\right]}{\sum_{p=0}^{n} \exp \left[-\left(E_{1 p}-E_{10}\right) / k_{B} T\right]} .
$$

For both equations, $E_{x y}$ represents the energy level of the $y$-th Stark component of the given state with energy $E_{x}$.

\section{Lifetime}

If the ions at the upper state shown in Figure 2.3 decays only by emission of photons, the relaxation rate of the upper state is the sum of the rates of all possible transitions to the lower state. The corresponding coefficient of spontaneous decay $A_{10}$ is equal to the reciprocal of the upper state's luminescent lifetime which is denoted by $\tau_{10}$ [23],

$$
A_{10}=\frac{1}{\tau_{10}} .
$$

The $\tau_{10}$ can be determined by measuring the rate of luminescence decay. It is defined as the time needed for the excited ions at a given energy level to decrease to the fraction $1 / \mathrm{e}$ of the original number of excited ions.

As the energy gap of the ${ }^{2} \mathrm{~F}_{5 / 2}$ upper state and the ${ }^{2} \mathrm{~F}_{7 / 2}$ ground state manifolds in $\mathrm{Yb}^{3+}$ is $\sim 10000 \mathrm{~cm}^{-1}$, non-radiative relaxation from the upper state is not probable. Therefore, it is often assumed that the measured luminescent lifetime represents the radiative lifetime $\tau_{\text {rad }}[82,83]$. There are, however, a number of experimental results [77] indicating that small amount of non-radiative relaxation may occur in $\mathrm{Yb}^{3+}$-doped media. 


\section{Atomic cross-section}

The atomic transition cross-section $\sigma_{\text {atom }}$, which represents the strength of transition, in a two-level system can be modeled using the expression $[22,23]$

$$
\sigma_{\text {atom }}(v)=S \gamma(v)
$$

where $v$ is the frequency. The term $\gamma(v)$ represents the spectral line-shape function. In the case of crystalline material, homogeneous broadening would dominate and $\gamma(v)$ is described by the Lorentzian function

$$
\gamma(v)=\frac{1}{\pi} \frac{\Delta v / 2}{\left(v-v_{0}\right)^{2}+(\Delta v / 2)^{2}},
$$

with

$$
\int \gamma(v) d v=1
$$

where $v_{0}$ is the center frequency and $\Delta v$ is the full width at half maximum (FWHM). Both parameters are temperature dependent due to the ion-host interactions [84]. A small frequency shift of $v_{0}$ with temperature is anticipated while $\Delta v$ is strongly temperature dependent.

$$
S=\int \sigma_{\text {atom }}(v) d v=\int \sigma_{\text {atom }}\left(v_{0}\right) \frac{\pi}{2} \Delta v \gamma(v) d v=\sigma_{\text {atom }}\left(v_{0}\right) \frac{\pi}{2} \Delta v
$$

is the integral transition cross-section [23], in units of $\mathrm{m}^{2} / \mathrm{s}$, which is independent of frequency and also independent of temperature [23]. Consequently, $\Delta v$ and $\sigma_{\text {atom }}\left(v_{0}\right)$ depend in opposite ways on temperature.

\section{Effective cross-sections}

The presence of Stark levels in the manifolds of rare-earth-ion-doped materials permits a combination of inter-band transitions which results in spectrally overlapped response. Consequently, the absorption cross-section $\sigma_{a b s}$ and the emission cross-section $\sigma_{e m}$ can be considered as the convolution of all possible transitions in the system $[84,85]$

$$
\begin{gathered}
\sigma_{a b s}(\lambda, T)=\sum_{i j} f_{0 i}(T) \sigma_{a t o m, i j}(\lambda, T), \\
\sigma_{e m}(\lambda, T)=\sum_{j i} f_{1 j}(T) \sigma_{a t o m, j i}(\lambda, T),
\end{gathered}
$$

where $f_{0 i}$ and $f_{1 j}$ are the relevant fractional populations defined by Eqs. 2.10 and 2.11.

The $\sigma_{a b s}$ and $\sigma_{e m}$ given in Eqs. 2.17 and 2.18 are the effective cross-sections taking into account the fractional population at a given temperature and they will be used throughout this thesis. 


\section{Temperature dependence of peak effective cross-sections}

Since the effective cross-sections are temperature dependent, the change of operating temperature could affect the performance of rare-earth-ion-doped devices. The knowledge on the influence of temperature on local peak values of the effective crosssection spectrum is particularly important because they are usually chosen as pump or signal wavelength.

Consider the case of effective absorption cross-section $\sigma_{a b s}$, in which the local peak absorption occurs at the central wavelength of the peak with a corresponding frequency $v=v_{0}$. The magnitude of the line-shape at $v=v_{0}$ is

$$
\gamma\left(v_{0}\right)=\frac{1}{\pi}\left(\frac{\Delta v}{2}\right)\left(\frac{2}{\Delta v}\right)^{2}=\frac{2}{\pi \Delta v},
$$

Consequently, the atomic cross-section is given by

$$
\sigma_{\text {atom }}\left(v_{0}\right)=S \cdot \frac{2}{\pi \Delta v} \text {. }
$$

Assuming that the observed absorption has negligible contribution from other transitions, the peak $\sigma_{a b s}$ can be described using only a single transition instead of the summation of all transitions,

$$
\sigma_{a b s}(\lambda, T) \approx f_{0 i}(T) \sigma_{\text {atom }}(\lambda, T) \approx f_{0 i}(T) S \frac{2}{\pi \Delta v}
$$

The strongest absorption usually corresponds to the transition starting from the lowest Stark level of the ground state, i.e. $i=0$, as the fractional population of this Stark level is the highest among all Stark levels. In this case, Eq. 2.21 can be written as

$$
\begin{gathered}
\sigma_{a b s}(T) \approx f_{00}(T) S \frac{2}{\pi \Delta v(T)}, \text { or } \\
\sigma_{a b s}(T) \propto f_{00}(T) / \Delta v(T) .
\end{gathered}
$$

Hence, once the peak $\sigma_{a b s}$ at a reference temperature $T_{0}$ (e.g. room temperature) is known, the peak $\sigma_{a b s}$ at arbitrary temperature can be approximated by

$$
\sigma_{a b s}(T)=\sigma_{a b s}\left(T_{0}\right) \frac{f_{00}(T)}{f_{00}\left(T_{0}\right)} \frac{\Delta v\left(T_{0}\right)}{\Delta v(T)} .
$$

Equation 2.24 signifies that the peak $\sigma_{a b s}$ changes with temperature according to the temperature dependence of (i) the Boltzmann factor of the starting Stark level, and (ii) the transition linewidth. Similar argument also holds for the peak $\sigma_{e m}$, where its temperature dependence can be derived using the abovementioned procedure. 


\subsubsection{Gain and loss in optically active media}

A light beam traversing in an optically active medium in $z$-direction may be partially absorbed or amplified by the medium. The change of intensity $I$ of the light beam per unit length follows the Lambert-Beer law,

$$
\frac{d I(\lambda, z)}{d z}=g(\lambda, T) I(\lambda, z),
$$

where $g(\lambda, T)$ is the small-signal gain coefficient in $\mathrm{cm}^{-1}$ at wavelength $\lambda$ and temperature $T . g(\lambda, T)$ is usually a spatially dependent parameter and it is related to the effective transition cross-section, $\sigma_{a b s}$ and $\sigma_{e m}$ via

$$
\begin{gathered}
g(\lambda, T)=\sigma_{e m}(\lambda, T) N_{1}-\sigma_{a b s}(\lambda, T) N_{0}, \text { and } \\
N_{0}+N_{1}=N_{d},
\end{gathered}
$$

where $\sigma_{e m}$ is the effective emission cross-section and $\sigma_{a b s}$ is the effective absorption cross-section as defined in previous sub-section. $N_{d}$ represents the number of active ions per unit volume in the medium.

Based on the above expression, it is apparent that an active medium under the condition of $g<0$ would induce an optical loss - the intensity of the light beam will decrease as it propagates along the medium. In order to achieve optical amplification, a net gain at the signal wavelength is necessary (i.e. $g>0$ ). This can be achieved by careful design of amplifier to ensure that the condition $\sigma_{e m} N_{1}>\sigma_{a b s} N_{0}$ applies throughout a large part of the amplifier.

\subsubsection{Theories for determining effective cross-sections}

In order to determine the effective absorption cross-section $\sigma_{a b s}$, Eqs. 2.25-2.27 can be simplified by considering a low beam intensity during the absorption loss measurement. Hence, the approximation $N_{0} \approx N_{d}$ applies, leading to

$$
\frac{d I(z)}{d z} \approx\left[-\sigma_{a b s}(\lambda, T) N_{d}\right] \cdot I(z) \approx \alpha(\lambda, T) I(z),
$$

where the absorption coefficient $\alpha$ can be determined from the measured loss and the sample's thickness. Hence, based on the measured $\alpha$ and $N_{d}$, the effective absorption cross-section $\sigma_{a b s}$ can be calculated with

$$
\sigma_{a b s}(\lambda, T)=\alpha(\lambda, T) / N_{d}
$$

The emission cross-section, on the other hand, can be determined with two methods, namely the reciprocity theory and the Füchtbauer-Ladenburg equation or from a gain measurement by use of Eqs. 2.25 and 2.26. 


\section{Reciprocity theory}

The reciprocity theory, also known as the McCumber theory [86], is based on principle of detailed balance using the equality $\sigma_{\text {atom, } i j}(v)=\sigma_{\text {atom, } j i}(v)$, as shown in Figure 2.3. The theory indicates that the effective cross-sections of an optical center in thermal equilibrium are related to each other at every single frequency. With the knowledge of electronic structure of the active ion in question, the emission cross-section $\sigma_{e m}$ spectrum can be deduced from the absorption cross-section $\sigma_{a b s}$ spectrum using reciprocity method, or vice versa, based on the following expression [87],

$$
\sigma_{e m}(\lambda, T)=\sigma_{a b s}(\lambda, T) \frac{Z_{0}}{Z_{1}} \exp \left(\frac{E_{z l}-h c / \lambda}{k_{B} T}\right),
$$

where $c$ is the speed of light. $E_{z l}$ is the energy at the so called zero-phonon line at wavelength denoted by $\lambda_{z l}$ and it is the energy difference between the lowest Stark levels of the ground state and the excited state, as labeled in Figure 2.3. $Z_{0}$ and $Z_{1}$ are the respective partition function for the ground state and the excited state defined by

$$
\begin{aligned}
& Z_{0}=\sum_{i}^{m} \exp \left[-\left(E_{0 i}-E_{00}\right) / k_{B} T\right] . \\
& Z_{1}=\sum_{j}^{n} \exp \left[-\left(E_{1 j}-E_{10}\right) / k_{B} T\right] .
\end{aligned}
$$

\section{Füchtbauer-Ladenburg theory}

The Füchtbauer-Ladenburg theory [88-91] provides a relationship between the radiative lifetime $\tau_{\text {rad }}$ of the active ion to its emission cross-section, which is derived from the relationship between the Einstein coefficients (Eq. 2.8). The Füchtbauer-Ladenburg equation can be written in the either frequency or wavelength domain

$$
\frac{1}{\tau_{\text {rad }}}=\frac{8 \pi n^{2}}{c^{2}} \int v^{2} \sigma_{e m}(v, T) d v=8 \pi n^{2} c \int \frac{\sigma_{e m}(\lambda, T)}{\lambda^{4}} d \lambda,
$$

where $n$ is the refractive index of the medium. Assuming that the wavelength range is not too broad, a mean wavelength can be used as the wavelength term in the denominator of the integral in Eq. 2.33. As the luminescent intensity $I(\lambda)$ is proportional to the emission cross-section, the same equation can be written as

$$
\sigma_{e m}(\lambda, T)=\frac{\bar{\lambda}^{4}}{8 \pi n^{2} c \tau_{\text {rad }}} \frac{I(\lambda, T)}{\int I(\lambda, T) d \lambda} .
$$




\section{Applicability of the reciprocity and Füchtbauer-Ladenburg theories for $\mathbf{Y b}^{3+}$ activated media}

Calculation of emission cross-section using the reciprocity theory relies on the absorption cross-section data which is based on experimental absorption data. Most of the time, the long-wavelength tail of the measured absorption is inevitably low. Hence, the data collected within this region would exhibit signal-to-noise ratio lower than the other spectral region. As the equation of the reciprocity method has an exponential dependency to the wavelength, the noise in the absorption data at the long-wavelength region will be magnified in the calculated emission data. Hence, the accuracy of the calculated emission cross-section at this region could be compromised.

The Füchtbauer-Ladenburg (F-L) equation, on the other hand, deduce the emission cross-section by relying on the knowledge of radiative lifetime and emission spectral response. An important underlying assumption of the theory is that the reabsorption of radiation can be neglected. Unfortunately, the $\mathrm{Yb}^{3+}$ activated media exhibit strong spectral overlap of luminescent and ground state absorption which causes severe reabsorption effect $[92,93]$. As a result, the measured luminescent lifetime is elongated and deviates from the intrinsic lifetime value [92, 93]. In addition, the measured emission response at the strongest transition line may be underestimated [94] as the reabsorption effect is enhanced with the higher transition cross-section. Data collection with complete suppression of reabsorption effect remains a great challenge till date. Attempt had been made to deduce the emission cross-section in YAG:Yb ${ }^{3+}$ using F-L equation by correcting for the reabsorption effect [94], but the resulting emission cross-section was still $\sim 20 \%$ different from the result determined using reciprocity method despite the rather involved measurement procedure.

In certain applications, it is important to determine the cross-section at both shortwavelength and long-wavelength regions as accurate as possible. For instance, for a quasi-four level laser, the pump typically operates near the short-wavelength region whereas the lasing wavelength lies at the long-wavelength region. In this case, the results from the reciprocity method and the F-L method can be combined [95]. An example is shown in Figure 2.4, where the results from the reciprocity method at the short-wavelength region and the results from the F-L method at the long-wavelength region are stitched together to provide reliable emission cross-section data over the entire wavelength range of concern.

Since the signal wavelength for the current work is located at the central absorption line and the pump wavelength is much shorter than the signal wavelength, it is suffix to use only the reciprocity method to determine the emission cross-sections. It should be noted that the emission cross-section deduced in this work at the longwavelength region is associated with measurement uncertainty. Therefore, the data is not suitable for applications such as lasers operating at wavelength $>1030 \mathrm{~nm}$. 


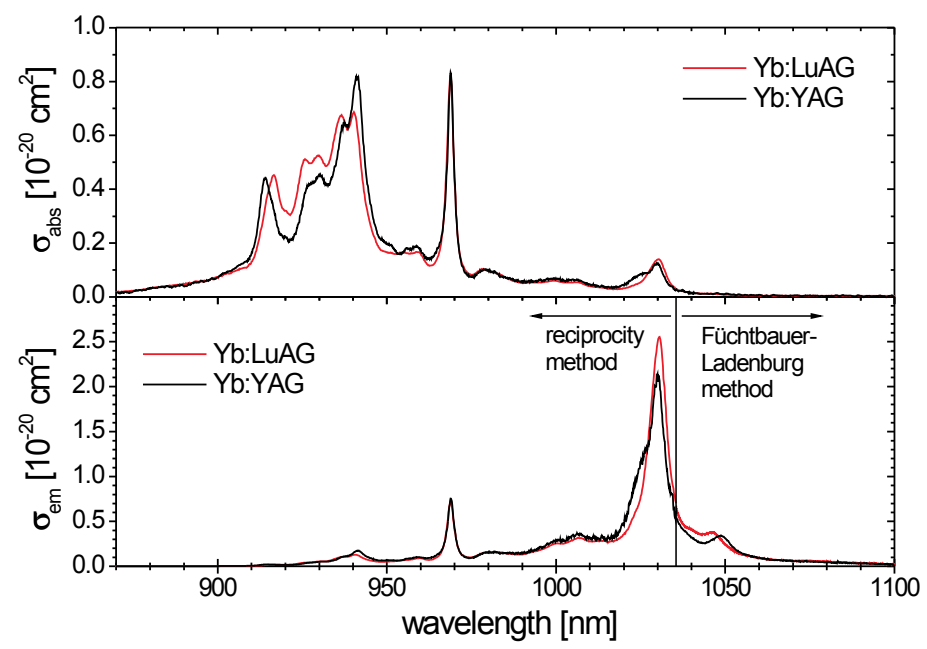

Figure 2.4 Emission cross-section results in LuAG: $\mathrm{Yb}^{3+}$ and YAG: $\mathrm{Yb}^{3+}$ obtained from combined results of the reciprocity method and the Füchtbauer-Ladenburg method (figure is reproduced from [95]). The calculated results from reciprocity method typically exhibit noise at the long-wavelength region as the noise from the absorption data is amplified by the exponential term in the equation. The measured emission data from $\mathrm{Yb}^{3+}$-doped material typically suffer from reabsorption effect, leading to underestimated cross-section value at the strongest transition line. In this figure, the emission data of both methods are stitched together to provide reliable emission cross-section data over the entire wavelength range. This is typically done by calibrating the two curves at certain wavelength region where both results are deemed reliable.

\subsection{Optical waveguiding}

An optical waveguide is a dielectric structure capable of confining electromagnetic wave within optical spectrum in one or more dimensions. It allows the optical beam to propagate with a fixed, and usually small, beam size. The benefit of waveguiding for rare-earth-ion-doped devices is illustrated in Figure 2.5. In the case of bulk crystal, high pump intensity is typically achieved by focusing the pump beam. However, a tightly focused beam would also diverge quickly, leading to non-ideal pumping condition in parts of the crystal. This imposes a bottom limit on the focused beam size in order to obtain optimal performance. Contrarily, waveguide structuring allows confinement of focused beam in lateral and transverse directions. Hence, high pump intensity can be maintained over a longer distance, which is limited by the length and the quality of the waveguide.

In what follows, the techniques used to fabricate optical waveguides in potassium double tungstates are discussed. The characteristics and the performance of different types of waveguide realized on potassium double tungstates will also be summarized. 


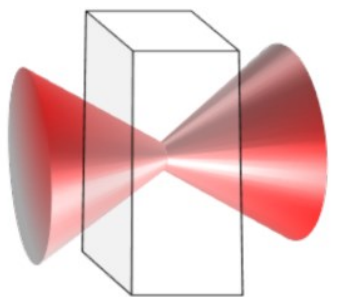

(a) rare-earth-doped bulk crystal

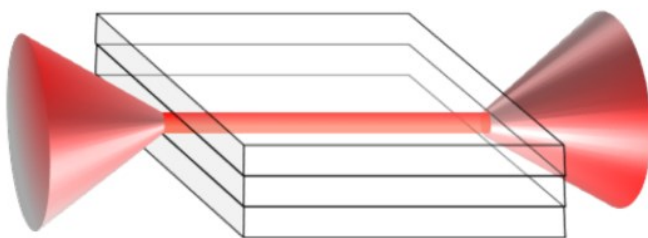

(b) rare-earth-doped waveguide

Figure 2.5 Visualization of a tightly focused optical pump beam propagating in (a) bulk crystal, and (b) optical waveguide. The former shows the beam diverges rapidly with propagation direction after the minimum beam waist while the later shows that the small focused beam size can be maintained over a relatively long distance.

\subsubsection{Waveguides based on potassium double tungstates}

The concept of rare-earth-ion-doped waveguide devices had been conceived for a long time. In fact, devices based on materials such as silica [44], $\mathrm{Al}_{2} \mathrm{O}_{3}$ [47], yttrium aluminum garnet (YAG) [96] had already been reported in the early 90s. Since the potassium double tungstate crystals began to receive considerable interest in the late $90 \mathrm{~s}$ [71], waveguide devices based on this material were only reported in the noughties.

The first waveguiding effect based on potassium double tungstates was reported by Romanyuk et al. in 2004 [61] in which guided luminescent out-coupled from a $2.5 \mu \mathrm{m}$ thick $\mathrm{KY}\left(\mathrm{WO}_{4}\right)_{2}: \mathrm{Tb}^{3+}(10$ at. \%) epitaxial layer was demonstrated. The use of planar waveguide for lasing purpose using $\mathrm{KY}\left(\mathrm{WO}_{4}\right)_{2}: \mathrm{Yb}^{3+}$ epitaxial layer * was reported in 2006 [62]. The results in [62] is reproduced in Figure 2.6, where a waveguiding effect in the layer was clearly shown.
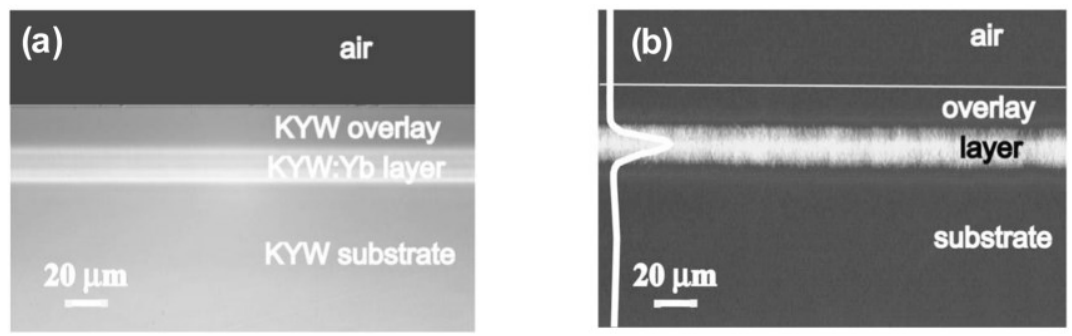

Figure 2.6 Demonstration of waveguiding effect on planar waveguide structure based on $22 \mu \mathrm{m}$ thick $\mathrm{KY}\left(\mathrm{WO}_{4}\right)_{2}: \mathrm{Yb}^{3+}$ epitaxial layer (figure is reproduced from [62]): (a) micrograph of the polished end surface, (b) guided $\mathrm{Yb}^{3+}$ luminescent and pump light in the planar waveguide and intensity distribution.

\footnotetext{
* The use of $\mathrm{KY}\left(\mathrm{WO}_{4}\right)_{2}: \mathrm{Yb}^{3+}$ epitaxial layer for lasing purpose had been reported by Aznar et al. in 2004 [60], but the cavity arrangement was such that the optical beams were propagating through the surface of the epitaxial layer, hence the laser demonstrated resembles a micro-chip laser rather than a waveguide laser.
} 
Improved performance on the active device can be achieved by imposing wave confinement in both vertical and horizontal directions which are transverse to the propagation direction. The reported potassium double tungstate waveguide structures which provide two dimensional wave confinement are shown in Figure 2.7. For the ease of discussion they will be referred based on the fabrication method: (a) ultra-fast laser writing [97, 98], (b) micro-structured epitaxy layer [99], and (c) epitaxial growth on micro-structured substrate [100].

The method (a) employs an ultrafast laser tightly focused into the material to induce a change of refractive index due to material strain. The method was introduced by Davis et al. [101] and it had been applied on a wide range of materials. Channel waveguide produced by this method on $\mathrm{KGd}\left(\mathrm{WO}_{4}\right)_{2}: \mathrm{Yb}^{3+}$ [97] and $\mathrm{KY}\left(\mathrm{WO}_{4}\right)_{2}: \mathrm{Yb}^{3+}$ $[97,98]$ had been reported. A double track writing is required as the process itself induces a reduction of index of refraction on the written track in potassium double tungstates. Figure 2.7(a) shows the cross-sectional view of a waveguide fabricated by ultrafast laser writing [97]. The bright circular beam spot shows a guided beam with reported spot size of $27 \mu \mathrm{m} \times 30 \mu \mathrm{m}$ between two laser written tracks. This method has an advantage that waveguides can be written on doped crystals available off the shelf.

Method (b) is similar to typical waveguide fabrication approach, where an epitaxy layer with index of refraction higher than that of the substrate is grown. The polished epitaxy layer is then micro-structured using lithography technique to form ridge structure, as shown in Figure 2.7(b). The benefit of this approach is that both the thickness of the epitaxial layer and the width of the ridge can be rather well controlled. Hence, spot size of the mode profile as small as $2.1 \mu \mathrm{m} \times 4.8 \mu \mathrm{m}$ can be achieved [99].

The method (c) [100] is similar to method (b), except on the layer selected for micro-structuring. The fabrication steps begin by micro-structuring of the substrate, which is followed by epitaxy growth of the waveguiding layer. The epitaxy layer is then planarized and an additional overgrown layer is added for a symmetrical refractive index profile. The resulting waveguide has an inverted triangular shape, as shown in Figure 2.7(c), resulting in an elliptical spot size of $8 \mu \mathrm{m} \times 47 \mu \mathrm{m}$.
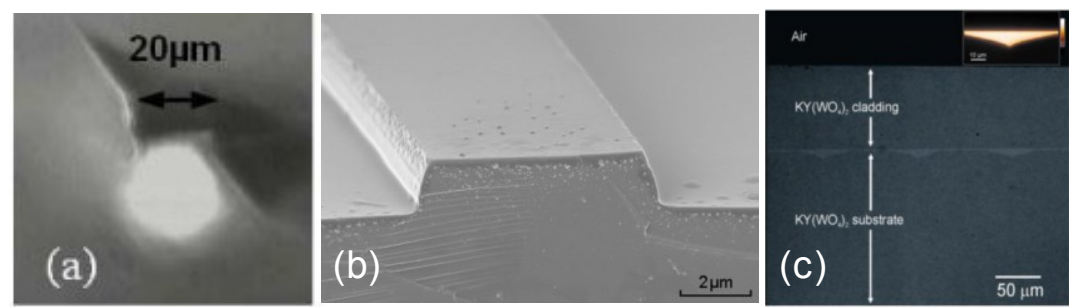

Figure 2.7 Cross-section view of waveguides based on potassium double tungstates fabricated by (a) ultrafast laser writing [97], (b) micro-structured epitaxy layer [99], and (c) epitaxial growth on micro-structured substrate [100]. 
A summary of the refractive index contrast with respect to the substrate and the spot size of the mode profile achieved with these methods can be found in Table 2.1.

Table 2.1 Comparison of the properties of waveguides based on potassium double tungstates fabricated using different methods.

\begin{tabular}{|l|c|c|}
\hline \multicolumn{1}{|c|}{ Method } & $\begin{array}{c}\text { Index } \\
\text { contrast }\end{array}$ & $\begin{array}{c}\text { Spot size } \\
(\boldsymbol{\mu m} \times \boldsymbol{\mu m})\end{array}$ \\
\hline (a) Ultrafast laser writing [97, 102] & $\sim 1 \times 10^{-3}$ & $27 \times 30$ \\
\hline (b) Micro-structured epitaxy layer [99] & $\sim 1 \times 10^{-2}$ & $2.1 \times 4.8$ \\
\hline (c) Epitaxial growth on micro-structured substrate [100] & $\sim 5 \times 10^{-3}$ & $8 \times 47$ \\
\hline
\end{tabular}

\subsection{Summary}

The characteristics of rare-earth elements, particularly those of $\mathrm{Yb}^{3+}$ ion, have been introduced in this chapter. Moreover, the theoretical background of the interaction of light and atomic system has been established. As the rare-earth ions exhibit Stark splitting in the manifolds, it is important to consider all possible inter-Stark interactions during the analysis of measurement results. Apart from defining the cross-sections for the transitions, relevant theories necessary for spectroscopic and optical gain investigations have been discussed. In addition, waveguide fabrication methods which had been applied on potassium double tungstates as well as the characteristics of the resulting waveguides have been reviewed. 



\section{Chapter 3}

\section{$\mathbf{Y b}^{3+}$-activated potassium double}

\section{tungstates}

\subsection{Overview}

The $\mathrm{Yb}^{3+}$-activated potassium double tungstates possess many qualities desirable for amplification and lasing applications. In this chapter, the characteristics of potassium double tungstates, including the crystallographic, optical, and thermal properties will be presented. Thereafter, the growth procedure used to produce the epitaxial layers used in this work will be introduced. Relevant measurement and processing methods applicable for all samples investigated in this thesis will be explained. Characterization results of the key samples used throughout this work will also be shown.

\subsection{Properties of $\mathrm{Yb}^{3+}$-doped potassium double tungstate bulk crystals}

Potassium rare-earth double tungstates $\mathrm{KRE}\left(\mathrm{WO}_{4}\right)_{2}$ (where $\mathrm{RE}=\mathrm{Gd}, \mathrm{Lu}, \mathrm{Y}$, or $\mathrm{Yb}$ ) incorporating trivalent ytterbium $\left(\mathrm{Yb}^{3+}\right)$ is known as an excellent gain medium due to its many favorable properties. The $\mathrm{KRE}\left(\mathrm{WO}_{4}\right)_{2}$ has a wide transparency window between $\sim 350 \mathrm{~nm}$ and $\sim 5000 \mathrm{~nm}$ [103-105]. The peak transition cross-sections in $\mathrm{KRE}\left(\mathrm{WO}_{4}\right)_{2}: \mathrm{Yb}^{3+}$ is $\sim 15$ times higher than those of $\mathrm{Yb}^{3+}$-doped yttrium aluminum garnet, YAG: $\mathrm{Yb}^{3+}[70,106]$. Besides, high-quality $\mathrm{KRE}\left(\mathrm{WO}_{4}\right)_{2}$ with $\mathrm{Yb}^{3+}$ up to 100 at.\%, i.e. $\mathrm{KYb}\left(\mathrm{WO}_{4}\right)_{2}$, can also be realized [104]. Many reports had shown that concentration dependent lifetime quenching on these high $\mathrm{Yb}^{3+}$ concentration crystals is very limited $[65,106,107]$. In addition, the $\mathrm{KRE}\left(\mathrm{WO}_{4}\right)_{2}: \mathrm{Yb}^{3+}$ has absorption band at $\sim 932 \mathrm{~nm}$ and $\sim 981 \mathrm{~nm}$, hence permitting the use of high brightness, high wall-plug efficiency InGaAs pump lasers [75]. From the mechanical point of view, the hardness of $\mathrm{KRE}\left(\mathrm{WO}_{4}\right)_{2}$ is $4-5$ on the Mohs scale, hence the crystal is sufficiently robust. The main disadvantage of $\mathrm{KRE}\left(\mathrm{WO}_{4}\right)_{2}$ is its rather modest thermal conductivity. The heat conduction in $\mathrm{KRE}\left(\mathrm{WO}_{4}\right)_{2}$ is about three times better than in silica fiber. However, it is inferior to those of the other popular laser crystals such as YAG and lutetium 
sesquioxide $\left(\mathrm{LuO}_{2}\right)$ [83]. Hence, $\mathrm{KRE}\left(\mathrm{WO}_{4}\right)_{2}$ is less suitable for high-average power systems [108].

\section{Crystallographic, optical, and thermal axes}

In order to maximize the potential gain of the material, it is important to distinguish the crystallographic, optical, and thermal axes in $\mathrm{KRE}\left(\mathrm{WO}_{4}\right)_{2}$, see Figure 3.1. The crystallographic axes of $\operatorname{KRE}\left(\mathrm{WO}_{4}\right)_{2}$ are represented by $\boldsymbol{a}, \boldsymbol{b}$, and $\boldsymbol{c}$. The optical axes which are orthogonal to each other are known as $N_{m}, N_{g}$, and $N_{p}$. The principle thermal axes are labeled as $X_{1}^{\prime}, X_{2}^{\prime}$, and $X_{3}^{\prime}$ and they are also orthogonal to each other. The $\boldsymbol{b}$, $N_{p}$, and $X_{2}^{\prime}$ are parallel to each other. In the coming sub-sections, relevant material properties will be discussed in further detail with reference to Figure 3.1.

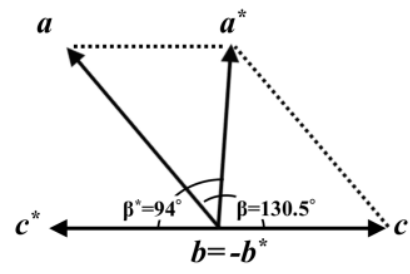

(a) Crystallographic

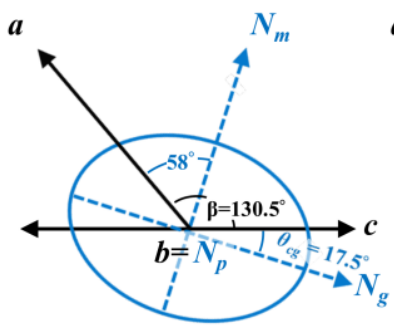

(b) Optical

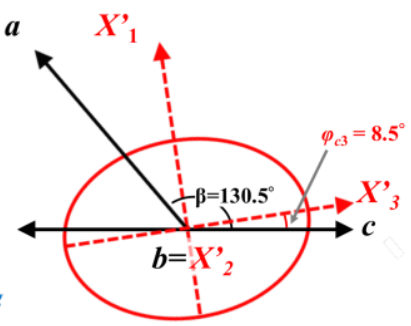

(c) Thermal

Figure 3.1 Overview of the relation between the crystallographic (black), optical (blue), and thermal (red) axes in $\mathrm{KRE}\left(\mathrm{WO}_{4}\right)_{2}$ : (a) The relationship between the basis vectors of the unit cell parameters in monoclinic $\mathrm{KRE}\left(\mathrm{WO}_{4}\right)_{2}$ expressed in the $C 2 / c$ space group $(a, b, c)$, and $I 2 / c$ space group $\left(a^{*},-b^{*}, c^{*}\right)$ settings [65, 103], (b) optical indicatrix $\left(N_{p}, N_{m}, N_{g}\right)$ and its relation with the basis vectors of $C 2 / c$, (c) principle thermal axes $\left(X_{1}^{\prime}, X_{2}{ }_{2}, X_{3}{ }_{3}\right)$ and its orientation with respect to the crystallographic axes. The $b$ and $-b^{*}$ (crystallographic), $N_{p}$ (optical), and $X_{2}^{\prime}$ (thermal) share a common axis which is perpendicular to the figure plane and directed towards the observer. The relative angles between the axes are taken from $\mathrm{KY}\left(\mathrm{WO}_{4}\right)_{2}[103,109,110]$. These angles are dependent on the type of $\mathrm{RE}$ and on the temperature.

\subsubsection{Crystallographic properties}

The focus of this study is on low-temperature phase of $\operatorname{KRE}\left(\mathrm{WO}_{4}\right)_{2}$, also known as $\alpha-\mathrm{KRE}\left(\mathrm{WO}_{4}\right)_{2}$, which has a monoclinic crystallographic structure and a high degree of anisotropy. The unit cell parameters of $\alpha-\operatorname{KRE}\left(\mathrm{WO}_{4}\right)_{2}$ can be expressed in either $C 2 / c$ or $I 2 / c$ space group, both belong to the centrosymmetric $2 / m$ point group $[65,103]$. The relationship between the unit vectors of $C 2 / c(\boldsymbol{a}, \boldsymbol{b}, \boldsymbol{c})$ and $I 2 / c\left(\boldsymbol{a}^{*}, \boldsymbol{b}^{*}, \boldsymbol{c}^{*}\right)$ space groups is shown in Figure 3.1(a). The lattice parameters for the unit cell of $\mathrm{KRE}\left(\mathrm{WO}_{4}\right)_{2}$ expressed in $C 2 / c$ space group is summarized in Table 3.1. The unit cell parameters are important consideration for the growth of high $\mathrm{Yb}^{3+}$ concentration epitaxial layers. A proper choice of the composition epitaxial layer or the substrate will ensure that the lattice matching condition between the layer and the substrate is fulfilled. This will be further discussed in Section 3.3. 
Table 3.1 Unit cell parameters of monoclinic $\mathrm{KGd}\left(\mathrm{WO}_{4}\right)_{2}, \mathrm{KLu}\left(\mathrm{WO}_{4}\right)_{2}, \mathrm{KY}\left(\mathrm{WO}_{4}\right)_{2}$ and $\mathrm{KYb}\left(\mathrm{WO}_{4}\right)_{2}$ expressed in $C 2 / c$ space group.

\begin{tabular}{|l|c|c|c|c|c|}
\hline Crystal & $\boldsymbol{a}(\boldsymbol{\AA})$ & $\boldsymbol{b}(\AA)$ & $\boldsymbol{c}(\boldsymbol{\AA})$ & $\left.\boldsymbol{\beta} \mathbf{(}^{\circ}\right)$ & Reference \\
\hline $\mathrm{KGd}\left(\mathrm{WO}_{4}\right)_{2}$ & $10.652(4)$ & $10.374(6)$ & $7.582(2)$ & $130.80(2)$ & {$[111]$} \\
\hline $\mathrm{KLu}\left(\mathrm{WO}_{4}\right)_{2}$ & $10.576(7)$ & $10.214(7)$ & $7.487(2)$ & $130.68(4)$ & {$[112]$} \\
\hline $\mathrm{KY}\left(\mathrm{WO}_{4}\right)_{2}$ & 10.64 & 10.35 & 7.54 & 130.5 & {$[103]$} \\
\hline $\mathrm{KY}\left(\mathrm{WO}_{4}\right)_{2}$ & $10.6313(4)$ & $10.3452(6)$ & $7.5547(3)$ & $130.752(2)$ & {$[109]$} \\
\hline $\mathrm{KYb}\left(\mathrm{WO}_{4}\right)_{2}$ & $10.590(4)$ & $10.290(6)$ & $7.478(2)$ & $130.70(2)$ & {$[104]$} \\
\hline
\end{tabular}

\subsubsection{Energy levels of $\mathbf{Y b}^{3+}$}

The $\mathrm{KGd}\left(\mathrm{WO}_{4}\right)_{2}: \mathrm{Yb}^{3+}, \mathrm{KLu}\left(\mathrm{WO}_{4}\right)_{2}: \mathrm{Yb}^{3+}, \mathrm{KY}\left(\mathrm{WO}_{4}\right)_{2}: \mathrm{Yb}^{3+}$, and $\mathrm{KYb}\left(\mathrm{WO}_{4}\right)_{2}$ have very similar energy levels $[71,73,74,104]$, with a characteristic total splitting of $\sim 550 \mathrm{~cm}^{-1}$ within the manifolds, as shown in Figure 3.2. Since the energy gap in between the split Stark levels within the manifolds are comparable to the thermal energy at room temperature $\left(k T \approx 200 \mathrm{~cm}^{-1}\right)$, the total population of the ions within a given manifold is distributed in accordance to the Boltzmann distribution (Eqs 2.10 and 2.11), represented by the blue horizontal thick bars in Figure 3.2.

The transitions of concern in this work is the upward transition $E_{00} \rightarrow E_{12}$ and the downward transition $E_{10} \rightarrow E_{00}$ corresponding to the pump wavelength of $\sim 932 \mathrm{~nm}$ and signal wavelength of $\sim 981 \mathrm{~nm}$. It shall be noted that a finite amount of population will reside at the Stark level with energy $E_{12}$ at and beyond $300 \mathrm{~K}$. This imposes a physical limit on the maximum population inversion achievable in the system. In other words, $100 \%$ population inversion will not occur regardless of the amount of pump power supplied to the system.

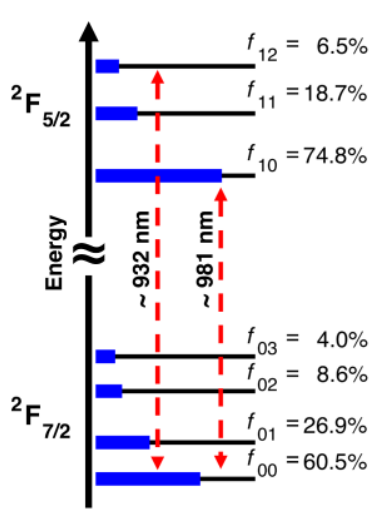

$$
\begin{aligned}
& \mathrm{KY}\left(\mathrm{WO}_{4}\right)_{2}: \mathrm{Yb}^{3+} \mathrm{KGd}\left(\mathrm{WO}_{4}\right)_{2}: \mathrm{Yb}^{3+} \mathrm{KLu}\left(\mathrm{WO}_{4}\right)_{2}: \mathrm{Yb}^{3+} \mathrm{KYb}\left(\mathrm{WO}_{4}\right)_{2} \\
& E_{12}=10695 \mathrm{~cm}^{-1} \quad 10686 \mathrm{~cm}^{-1} \quad 10735 \mathrm{~cm}^{-1} \quad 10734 \mathrm{~cm}^{-1} \\
& E_{11}=10476 \mathrm{~cm}^{-1} \quad 10487 \mathrm{~cm}^{-1} \quad 10498 \mathrm{~cm}^{-1} \quad 10500 \mathrm{~cm}^{-1} \\
& E_{10}=10187 \mathrm{~cm}^{-1} \quad 10196 \mathrm{~cm}^{-1} \quad 10187 \mathrm{~cm}^{-1} \quad 10188 \mathrm{~cm}^{-1} \\
& E_{03}=568 \mathrm{~cm}^{-1} \quad 535 \mathrm{~cm}^{-1} \quad 559 \mathrm{~cm}^{-1} \quad 555 \mathrm{~cm}^{-1} \\
& E_{02}=407 \mathrm{~cm}^{-1} \quad 385 \mathrm{~cm}^{-1} \quad 435 \mathrm{~cm}^{-1} \quad 438 \mathrm{~cm}^{-1} \\
& E_{01}=169 \mathrm{~cm}^{-1} \quad 163 \mathrm{~cm}^{-1} \quad 175 \mathrm{~cm}^{-1} \quad 168 \mathrm{~cm}^{-1} \\
& E_{00}=0 \mathrm{~cm}^{-1} \quad 0 \mathrm{~cm}^{-1} \quad 0 \mathrm{~cm}^{-1} \quad 0 \mathrm{~cm}^{-1}
\end{aligned}
$$

Figure 3.2 Energy-level diagram for $\mathrm{Yb}^{3+}$ in various potassium rare-earth double tungstates $[71,73,74,104]$. The thick horizontal bars in blue represent the estimated fractional populations within the upper $\left({ }^{2} F_{5 / 2}\right)$ and lower $\left({ }^{2} F_{7 / 2}\right)$ manifolds at $300 \mathrm{~K}$, calculated using the level energies of $\mathrm{KY}\left(\mathrm{WO}_{4}\right)_{2}: \mathrm{Yb}^{3+}[71]$. $E$ represents the energy level and $f_{x y}$ denotes the fractional population of the manifold at the Stark level with energy $E_{x y}$. 


\subsubsection{Optical indicatrix}

$\mathrm{KRE}\left(\mathrm{WO}_{4}\right)_{2}$ is biaxial crystal with three principle refractive index axes orthogonal to each other which are defined as $N_{p}, N_{m}$, and $N_{g}$, whereby the corresponding refractive index values exhibit the trend of $n_{p}<n_{m}<n_{g}{ }^{*} . N_{p}$ is in parallel to $\boldsymbol{b}$ crystallographic axis, whereas $N_{m}-N_{g}$ is at the same plane as $\boldsymbol{a}-\boldsymbol{c}$ crystallographic plane, see Figure 3.1(b). The orientation between sets of crystallographic and optical axes can be defined by the angle between crystallographic $c$ and optical $N_{g}$ axes, which is denoted here by $\theta_{c g}$. The reported $\theta_{c g}$ value for the respective $\mathrm{KRE}\left(\mathrm{WO}_{4}\right)_{2}$ is given in Table 3.2. In overall, the $\theta_{c g}$ is within the range of $17.5^{\circ}-21.5^{\circ}$.

The refractive index values for $\mathrm{KY}\left(\mathrm{WO}_{4}\right)_{2}$ at $N_{p}, N_{m}$, and $N_{g}$ axes obtained from [103] are shown in Figure 3.3(a). The dispersion curves in the same figure are plotted based on the Sellmeier equation fitted to the data [103]. The data in the figure shows a distinct trend of $n_{p}<n_{m}<n_{g}$. Among these axes, the $N_{m}$ corresponds to the polarization where the transition cross-sections are highest. Therefore, it is used more often for amplifiers and lasers in comparison to both $N_{g}$ and $N_{p}$ axes. The dispersion curves at $N_{m}$ for $\mathrm{KGd}\left(\mathrm{WO}_{4}\right)_{2}$ [113], $\mathrm{KLu}\left(\mathrm{WO}_{4}\right)_{2}$ [112], $\mathrm{KY}\left(\mathrm{WO}_{4}\right)_{2}$ [103], and $\mathrm{KYb}\left(\mathrm{WO}_{4}\right)_{2}$ [104] are depicted in Figure 3.3(b). Among these double tungstate crystals, the $\mathrm{KY}\left(\mathrm{WO}_{4}\right)_{2}$ exhibits the lowest refractive index value while the $\mathrm{KYb}\left(\mathrm{WO}_{4}\right)_{2}$ has the highest value, with two of them differ by $\sim 0.029$ at $1000 \mathrm{~nm}$ wavelength. With a proper choice of material composition, $\mathrm{KY}_{1-x-y-z} \mathrm{Gd}_{x} \mathrm{Lu}_{y} \mathrm{Yb}_{z}\left(\mathrm{WO}_{4}\right)_{2}$ epitaxial layers with $\mathrm{Yb}^{3+}$ concentration up to 47.5 at.\% grown on $\mathrm{KY}\left(\mathrm{WO}_{4}\right)_{2}$ substrate can result in refractive index contrast of $\sim 0.012$ at $N_{m}[65]$ which permits optical waveguiding in the layer.
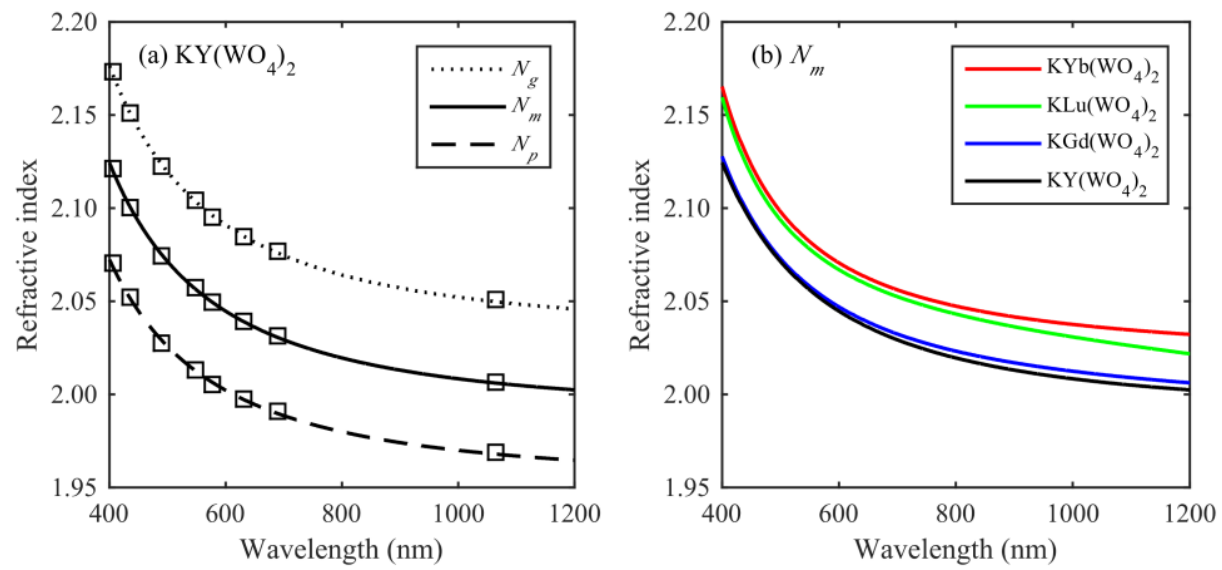

Figure 3.3 Dispersion curves for (a) $\mathrm{KY}\left(\mathrm{WO}_{4}\right)_{2}$ at $N_{g}, N_{m}$, and $N_{p}$ optical axes [103] (the squares are measurement points and the lines are plotted based on fitted Sellmeier equation), and (b) $\mathrm{KGd}\left(\mathrm{WO}_{4}\right)_{2}[113], \mathrm{KLu}\left(\mathrm{WO}_{4}\right)_{2}[112], \mathrm{KY}\left(\mathrm{WO}_{4}\right)_{2}[103]$, and $\mathrm{KYb}\left(\mathrm{WO}_{4}\right)_{2}$ at $N_{m}$ optical axis.

\footnotetext{
* $g$ represents 'grand', $m$ represents 'medium', whereas $p$ represents 'petit'.
} 
Table 3.2 Orientation between crystallographic and principle refractive axes in $\mathrm{KRE}\left(\mathrm{WO}_{4}\right)_{2}$.

\begin{tabular}{|l|c|c|}
\hline Crystal & $\left.\boldsymbol{\theta}_{\boldsymbol{c g}} \mathbf{(}^{\circ}\right)^{*}$ & Reference \\
\hline $\mathrm{KGd}\left(\mathrm{WO}_{4}\right)_{2}$ & $21.5 \pm 1.0$ & {$[73,113]$} \\
\hline $\mathrm{KGd}\left(\mathrm{WO}_{4}\right)_{2}$ & 19.3 & {$[114]$} \\
\hline $\mathrm{KLu}\left(\mathrm{WO}_{4}\right)_{2}$ & 18.5 & {$[112]$} \\
\hline $\mathrm{KY}\left(\mathrm{WO}_{4}\right)_{2}$ & $17.5 \pm 0.5$ & {$[103]$} \\
\hline $\mathrm{KY}\left(\mathrm{WO}_{4}\right)_{2}$ & 18.5 & {$[105]$} \\
\hline $\mathrm{KYb}\left(\mathrm{WO}_{4}\right)_{2}$ & 19 & {$[104]$} \\
\hline
\end{tabular}

* $\theta_{c g}$ represents the angle from $c$ crystallographic axis to $N_{g}$ optical axis in clockwise direction.

\subsubsection{Thermal properties}

The thermal conductivity in $\mathrm{KRE}\left(\mathrm{WO}_{4}\right)_{2}$ is also anisotropic, as in the case of the optical indicatrix, since its crystal structure has low symmetry. The thermal axes in $\mathrm{KRE}\left(\mathrm{WO}_{4}\right)_{2}$ is characterized by mutually orthogonal $X^{\prime}{ }_{1}, X^{\prime}{ }_{2}$, and $X^{\prime}{ }_{3}$, where the lowest thermal conductivity lies on the $X_{2}^{\prime}$ axis. As indicated in Figure 3.1(c), $X_{2}^{\prime}$ is in parallel with the crystallographic axis $\boldsymbol{b}$ and the optical axis $N_{p}$, whereas $X_{1}^{\prime}$ and $X_{3}^{\prime}$ are in the same plane as the $\boldsymbol{a}-\boldsymbol{c}$ or $N_{g}-N_{m}$ plane. The orientation of the $X^{\prime}{ }_{3}$ is $\sim 8.5-13.5^{\circ}$ counterclockwise from $c$. The respective angle between the $c$ and $X^{\prime}{ }_{3}$ axes, which is denoted as $\varphi_{c 3}$, for different types of double tungstates is listed in Table 3.3. It should be noted that the orientation of the thermal ellipsoid deviates slightly with the change of temperature $[115,116]$.

The density of the material $\rho$, the specific heat capacity $C_{p}$, and the thermal conductivity $k$ are often required for thermal analysis using heat equation. The values of these parameters in $\mathrm{KRE}\left(\mathrm{WO}_{4}\right)_{2}$ are collected and tabulated in Table 3.3. Generally, a high thermal conductivity is desired to efficiently dissipate the heat generated in the pumped region to the remaining body of the gain medium. The thermal conductivity in $\mathrm{KRE}\left(\mathrm{WO}_{4}\right)_{2}$ is $\sim 3 \mathrm{Wm}^{-1} \mathrm{~K}^{-1}$, which is higher than those of phosphate glass $\left(0.8 \mathrm{Wm}^{-1} \mathrm{~K}^{-1}\right)$ [117] and silica fibers but lower than those of crystalline $\mathrm{Yb}^{3+}$ hosts, such as YLF $\left(5.3 \mathrm{Wm}^{-1} \mathrm{~K}^{-1}\right)$, LuAG $\left(8.3 \mathrm{Wm}^{-1} \mathrm{~K}^{-1}\right)$, and YAG $\left(11.2 \mathrm{Wm}^{-1} \mathrm{~K}^{-1}\right)$ [118].

The thermal conductivity in YAG was shown to decrease from $11.2 \mathrm{Wm}^{-1} \mathrm{~K}^{-1}$ to 6.7 $\mathrm{Wm}^{-1} \mathrm{~K}^{-1}$ with the change of $\mathrm{Yb}^{3+}$ concentration from an undoped $\mathrm{YAG}$ up to 15 at.\% $\mathrm{Yb}^{3+}$-doped YAG [118]. Similar trend had also been observed in $\mathrm{YAlO}_{3}$ [118]. The decrease of thermal conductivity with increasing $\mathrm{Yb}^{3+}$ concentration could be due to the change of lattice vibration modes which arises from different crystal symmetry [119]. A separate study on YAG: $\mathrm{Yb}^{3+}$ shows that the thermal conductivity decreases with the increase of $\mathrm{Yb}^{3+}$ doping up to 50 at.\% but the thermal conductivity of stoichiometric $\mathrm{YbAG}$ (i.e. $100 \% \mathrm{Yb}^{3+}$ ) is higher than heavily doped samples and is comparable to $\mathrm{YAG}: \mathrm{Yb}^{3+}(15$ at.\%) [120]. Comparison of the data between undoped 
$\mathrm{KLu}\left(\mathrm{WO}_{4}\right)_{2}$ [115] and $\mathrm{Yb}^{3+}$-doped $\mathrm{KLu}\left(\mathrm{WO}_{4}\right)_{2}$ [116] (see Table 3.3) seems to suggest that incorporating $\mathrm{Yb}^{3+}$ increases the thermal conductivity. Nevertheless, it is probable that the thermal conductivity values of the high $\mathrm{Yb}^{3+}$ concentration $\mathrm{KRE}\left(\mathrm{WO}_{4}\right)_{2}$ samples used in this work would be lower than the thermal conductivity of low $\mathrm{Yb}^{3+}$ concentration $\mathrm{KLu}\left(\mathrm{WO}_{4}\right)_{2}$ [116] shown in Table 3.3.

Table 3.3 Orientation angle $\varphi$, material density $\rho$, specific heat capacity $C_{p}$, thermal conductivity $k$, and the temperature at which the data is collected $T$ for $\operatorname{KRE}\left(\mathrm{WO}_{4}\right)_{2}$.

\begin{tabular}{|l|c|c|c|c|c|c|}
\hline Crystal & $\left.\boldsymbol{\varphi}_{\mathbf{c} 3} \mathbf{(}^{\circ}\right)^{*}$ & $\boldsymbol{\rho}\left(\mathbf{g} / \mathbf{c m}^{\mathbf{3}}\right)$ & $\boldsymbol{C}_{\boldsymbol{p}}\left(\mathbf{J g}^{-1} \mathbf{K}^{-1}\right)$ & $\boldsymbol{k}\left(\mathbf{W m}^{-1} \mathbf{K}^{-1}\right)$ & $\mathbf{T}(\mathbf{K})$ & Reference \\
\hline $\mathrm{KGd}\left(\mathrm{WO}_{4}\right)$ & 12 & 7.11 & 0.363 & $k_{2}=2.6$ & 298 & {$[111,118]$} \\
\hline & 13.51 & 7.686 & 0.324 & $\begin{array}{l}k_{1}=2.95 \\
k_{2}=2.36 \\
k_{3}=4.06\end{array}$ & 300 & {$[112,115]$} \\
$\mathrm{KLu}\left(\mathrm{WO}_{4}\right)_{2}$ & & & & & \\
& & & & & & \\
& - & 7.637 & 0.365 & $k_{1}=3.09$ & 298.15 & {$[116]$} \\
$\mathrm{KLu}\left(\mathrm{WO}_{4}\right)_{2}: \mathrm{Yb}^{3+}$ & & & $(363.15 \mathrm{~K})$ & $\begin{array}{l}k_{2}=2.55 \\
k_{3}=4.40\end{array}$ & & \\
\hline $\mathrm{KY}\left(\mathrm{WO}_{4}\right)_{2}$ & 8.5 & 6.57 & 0.397 & $k_{2}=2.7$ & 298 & {$[110,118]$} \\
\hline $\mathrm{KYb}\left(\mathrm{WO}_{4}\right)_{2}$ & 12 & 7.610 & 0.324 & - & 298 & {$[104,115]$} \\
\hline
\end{tabular}

${ }^{*} \varphi_{\mathrm{c} 3}$ represents the angle from $c$ axis to $X_{3}^{\prime}$ thermal axis in counter-clockwise direction.

${ }^{* *} k_{1}, k_{2}$, and $k_{3}$ are the thermal conductivity values for $X^{\prime}{ }_{1}, X^{\prime}{ }_{2}$, and $X^{\prime}{ }_{3}$ axes, respectively.

\section{3 $\mathrm{Yb}^{3+}$-activated potassium double tungstate epitaxial layers}

In order to realized high $\mathrm{Yb}^{3+}$ concentration epitaxial layer of high quality, it is important to account for all the aspects which would affect the growth. Based on the knowledge on the crystallographic, optical, and thermal properties of bulk $\mathrm{KRE}\left(\mathrm{WO}_{4}\right)_{2}$ crystals, several key considerations for the growth of high $\mathrm{Yb}^{3+}$ concentration epitaxial layers are listed as follows:

Growth consideration. From the epitaxial growth point of view, the $\boldsymbol{b}$-orientation of $\mathrm{KRE}\left(\mathrm{WO}_{4}\right)_{2}$ has the slowest growth rate as compared to other crystallographic orientations, hence it is the most favorable orientation to achieve a stable growth on the crystal face. Therefore, all substrates used in this work were diced and polished such that they exhibit largest surface perpendicular to the $\boldsymbol{b}$-crystallographic direction.

Waveguiding consideration. In order to provide a condition for waveguiding, the epitaxial layer must possess a refractive index higher than its surrounding media. The stoichiometric $\mathrm{KYb}\left(\mathrm{WO}_{4}\right)_{2}$ crystal has the highest index of refraction among the double tungstates, as shown in Figure 3.3(b). Therefore, incorporation of $\mathrm{Yb}^{3+}$ in $\mathrm{KGd}\left(\mathrm{WO}_{4}\right)_{2}$, $\mathrm{KLu}\left(\mathrm{WO}_{4}\right)_{2}$, or $\mathrm{KY}\left(\mathrm{WO}_{4}\right)_{2}$ is likely to increase refractive index in the epitaxial layer. 
Assuming a linear dependency of the increase of refractive index to the amount of $\mathrm{Yb}^{3+}$, the highest refractive index contrast would be achievable using $\mathrm{KY}\left(\mathrm{WO}_{4}\right)_{2}$ substrate, whereas the lowest refractive index contrast is expected if $\mathrm{KLu}\left(\mathrm{WO}_{4}\right)_{2}$ substrate was used.

Thermal consideration. The choice of substrate material is not expected to severely affect the device performance because different types of $\mathrm{KRE}\left(\mathrm{WO}_{4}\right)_{2}$ exhibit rather similar thermal conductivity (see Table 3.3) and the limiting factor for thermal build-up in the gain medium is often due to efficiency of heat extraction from the crystal instead. Having said that, the thermal expansion of the unit cells should be taken into account during the growth process as the process takes place at temperature $>900{ }^{\circ} \mathrm{C}$. In order to avoid thermal shock, the furnace is usually cooled down in a very slow rate after the epitaxial growth before removing the sample.

Taking into account the above considerations, two approaches shown in Figure 3.4 were adopted to grow high $\mathrm{Yb}^{3+}$ concentration epitaxial layer. The first one employs a lattice engineering approach to grow $\mathrm{KY}_{1-x-y-z} \mathrm{Gd}_{x} \mathrm{Lu}_{y} \mathrm{Yb}_{z}\left(\mathrm{WO}_{4}\right)_{2}$ epitaxial layer lattice matched to the undoped $\mathrm{KY}\left(\mathrm{WO}_{4}\right)_{2}$ substrate $[64,65]$. In order to accommodate high amount of $\mathrm{Yb}^{3+}$, the lattice parameters of the epitaxial layer were optimized by codoping with optically inert $\mathrm{Gd}^{3+}$ and $\mathrm{Lu}^{3+}$ ions to minimize the lattice mismatch in $\boldsymbol{a}$ and $c$ crystallographic directions. The second approach uses $\mathrm{KLu}\left(\mathrm{WO}_{4}\right)_{2}$ as substrate and the high $\mathrm{Yb}^{3+}$-containing layer is grown by substituting the $\mathrm{Lu}^{3+}$ with $\mathrm{Yb}^{3+}$ [107]. This approach exploits the closely matched unit cell parameters between $\mathrm{KLu}\left(\mathrm{WO}_{4}\right)_{2}$ and $\mathrm{KYb}\left(\mathrm{WO}_{4}\right)_{2}$ to produce thick and high $\mathrm{Yb}^{3+}$ concentration epitaxial layers [121]. However, the refractive index contrast of epitaxial layers grown with this approach is in the order of $1 \times 10^{-3}$ at $N_{m}$ polarization [121], hence it is less favorable for waveguide amplifier purpose.

(a)

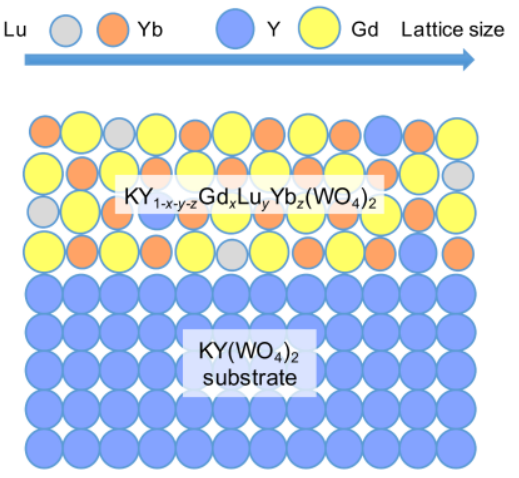

(b)

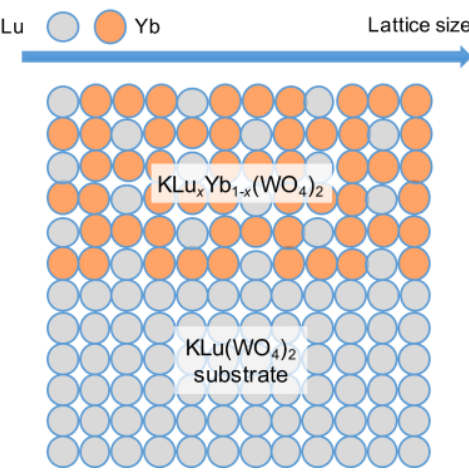

Figure 3.4 Two configurations of high $\mathrm{Yb}^{3+}$ concentration epitaxial film investigated in this work: (a) $\mathrm{KY}_{1-x-y-z} \mathrm{Gd}_{x} L u_{y} \mathrm{Yb}_{z}\left(\mathrm{WO}_{4}\right)_{2}$ epitaxial layer grown on undoped $\mathrm{KY}\left(\mathrm{WO}_{4}\right)_{2}$ substrate, and (b) $\mathrm{KLu}_{\mathrm{x}} \mathrm{Yb}_{1-\mathrm{x}}\left(\mathrm{WO}_{4}\right)_{2}$ epitaxial layer grown on undoped $\mathrm{KLu}\left(\mathrm{WO}_{4}\right)_{2}$ substrate. 


\subsubsection{Growth of epitaxial layer}

The epitaxial layers used in this thesis were grown by Dr. Shanmugam Aravazhi in Integrated Optical MicroSystems group at University of Twente unless mentioned otherwise. $\mathrm{KY}_{1-x-y-z} \mathrm{Gd}_{x} \mathrm{Lu}_{y} \mathrm{Yb}_{z}\left(\mathrm{WO}_{4}\right)_{2}$ layers were grown onto commercially available 1-mm-thick, $\boldsymbol{b}$-oriented, and polished $\mathrm{KY}\left(\mathrm{WO}_{4}\right)_{2}$ substrates (Altechna) by liquid phase epitaxy (LPE) using a $\mathrm{K}_{2} \mathrm{~W}_{2} \mathrm{O}_{7}$ solvent contained within a 25 -ml-volume platinum crucible at $920-925^{\circ} \mathrm{C}$ [65]. The solute/solvent ratio was $10.5-89.5 \mathrm{~mol} \%$. An in-house developed LPE-growth system with the growth temperature controlled within $\pm 0.1{ }^{\circ} \mathrm{C}$ shown in Figure 3.5 was used. It consists of a resistance-heated oven (Carbolite) with a single-zone vertical temperature profile at ambient atmosphere. All samples were grown using $5 \mathrm{~N}$ raw materials (Alfa Aesar). The samples which exhibit the highest $\mathrm{Yb}^{3+}$ concentration have a nominal composition of $\mathrm{KGd}_{0.425} \mathrm{Yb}_{0.575}\left(\mathrm{WO}_{4}\right)_{2}$.

The $\mathrm{KLu}_{x} \mathrm{Yb}_{1-x}\left(\mathrm{WO}_{4}\right)_{2}$ layer with a nominal $\mathrm{Yb}^{3+}$ concentration of 75 at.\% grown onto a 2-mm-thick $\mathrm{KLu}\left(\mathrm{WO}_{4}\right)_{2}$ substrate was supplied by Dr. Joan J. Carvajal from Universitat Rovira i Virgili (URV), Tarragona. The $\boldsymbol{b}$-oriented substrate was homegrown by top-seeded solution growth [107]. A solution with a solute:solvent ratio of 7:93 mol\% was used to grow the epitaxial layer [122]. The $\mathrm{KLu}\left(\mathrm{WO}_{4}\right)_{2}$ substrate was partially immersed into the solution at saturation temperature by vertical dipping. Immediately after immersion, the temperature of the solution was decreased by $3 \mathrm{~K}$ below the saturation temperature and the epitaxial growth process was undertaken at this temperature for 3 hours. Finally, the substrate was removed from the solution and the furnace was cooled to room temperature at $15 \mathrm{~K} /$ hour to prevent cracking of the structures.

(a)

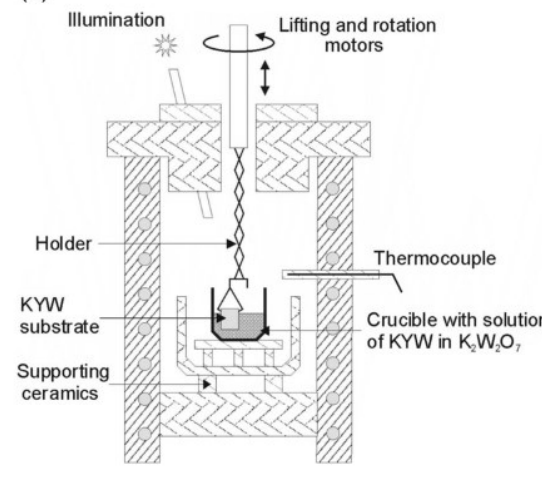

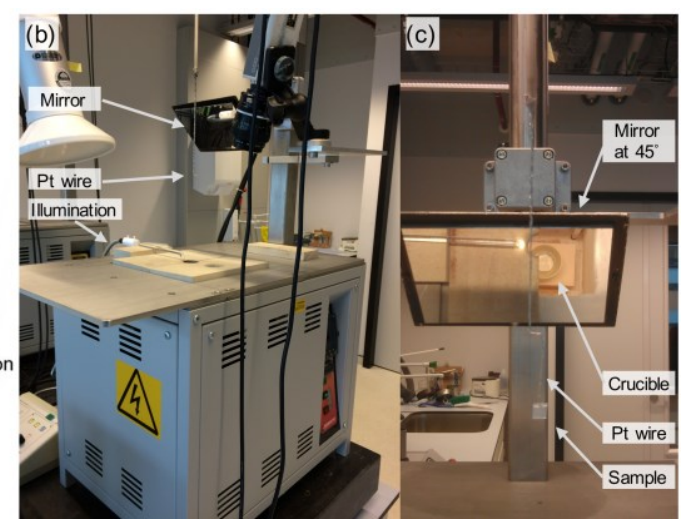

Figure 3.5 (a) Schematic diagram of the liquid phase epitaxy (LPE) system used to grow the high $\mathrm{Yb}^{3+}$ concentration layers on $\mathrm{KY}\left(\mathrm{WO}_{4}\right)_{2}$ [63]. (b) Photograph of the LPE system. A mirror is mounted at $45^{\circ}$ for the ease of observation while introducing sample into the oven or removing sample out of oven. (c) Close up view of the LPE system prior to an actual growth experiment. The sample was hooked to the platinum wire and was about to be translated down into the oven. The reflection from the monitoring mirror mounted at $45^{\circ}$ shows the platinum crucible containing the growth solution. 


\subsubsection{Lapping and polishing process}

Lapping and polishing steps needed to produce epitaxial layers with uniform thickness were done mechanically using a polishing equipment (Logitech PM-5) shown in Figure 3.6(a). Prior to each polishing trial, the sample was mounted on a chuck using quartz wax as displayed in Figure 3.6(b). The chuck was screwed onto a polishing jig, and an autocollimator (Logitech LG1/LG2) was used to check the orientation of the sample's surface. Since the substrate was not fully dipped into the solution during the growth process, the remaining area of the substrate with no epitaxial layer was used as reference surface. The planarity control knobs on the jig were adjusted to ensure that the polished surface was parallel to the substrate's surface.

Sample lapping was performed on a cast iron disk using aluminum oxide slurry with $3 \mu \mathrm{m}$ grain size. Typical lapping duration was about 5-15 minutes by exerting a low pressure of $\sim 50 \mathrm{~g} / \mathrm{cm}^{2}$ onto the sample, depending on the as-grown layer thickness and the target layer thickness. After thoroughly cleaning the sample and the polishing equipment, sample polishing was performed on polyurethane coated disk using Struers OP-U colloidal silica suspension. As the high $\mathrm{Yb}^{3+}$ concentration epitaxial layers exhibit considerably amount of strain, the pressure exerted on the sample was reduced to $\sim 100 \mathrm{~g} / \mathrm{cm}^{2}$ as compared to typical setting of $\sim 400 \mathrm{~g} / \mathrm{cm}^{2}$ to prevent sample cracking. As a result, typical polishing duration was extended to 4-6 hours, as compared to 2-3 hours under normal pressure setting, before a good surface quality was obtained.
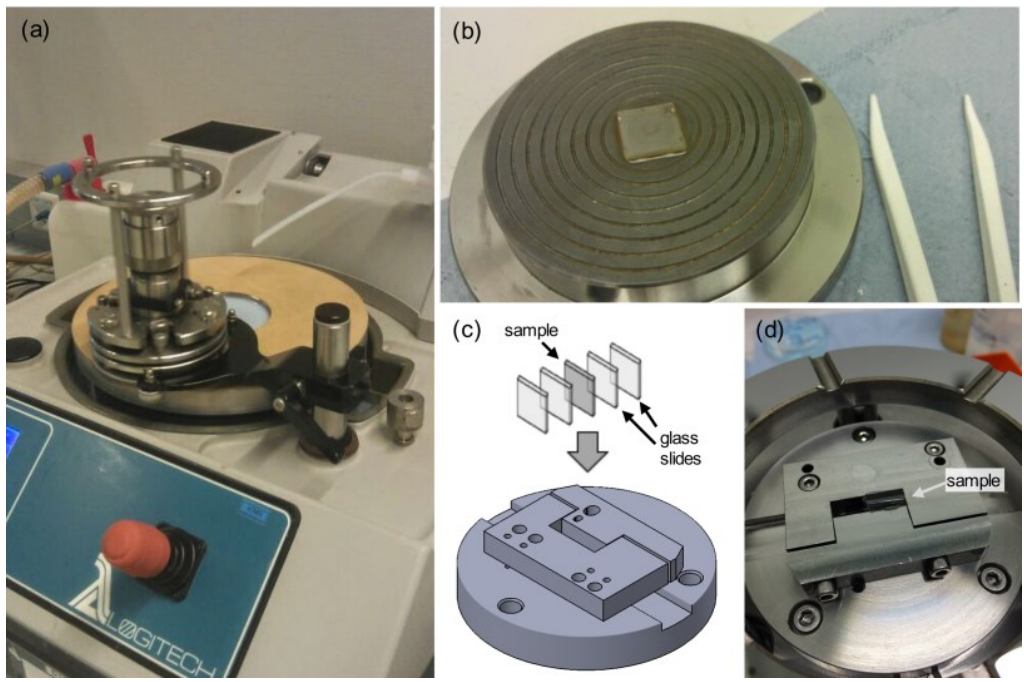

Figure 3.6 (a) Photograph showing the stainless steel jig placed on a rotating disc during the polishing process. (b) Photograph of a $10 \mathrm{~mm} \times 10 \mathrm{~mm} \times 1 \mathrm{~mm}$ sample with epitaxial layer mounted on a stainless steel polishing chuck using a small amount of quartz wax. (c) Illustration of sample mounting step for end-face polishing. The sample is sandwiched in between soda lime microscope slides using wax and the stack is clamped by a home-built sample holder. (d) Photograph of the sample mounted on holder, which was in turned screwed onto the jig. 
The thickness of the polished epitaxial layer was monitored with an interference microscope (Leitz). Figure 3.7(a) shows the interference microscope equipped with a filtered light source at $550 \mathrm{~nm}$ wavelength. A sample with polished epitaxial layer is shown next to it. As the sample was not fully dipped during the growth, parts of the top edge of the sample were not grown with epitaxial layer. The top center part of the sample was used as the reference for planarity adjustment of the polishing jig while the step-like interface circled in Figure 3.7(b) was used for thickness measurement. Typical fringes pattern observed on the microscope is exemplified in Figure 3.7(c). The distance between pairs of lines corresponds to half-wavelength of the light source. Hence, the thickness of the layer can be calculated by evaluating the shift of the fringes.

The end-face polishing was performed using the procedure similar to the surface polishing. The diced sample was stacked in between soda lime microscope slides by applying a thin layer of wax in between. The stack was then clamped using a homebuilt stainless steel holder, indicated by Figure 3.6(c) and Figure 3.6(d). It is important to ensure that the wax layers next to the sample are as thin as possible to minimize rounding of sample's edges.

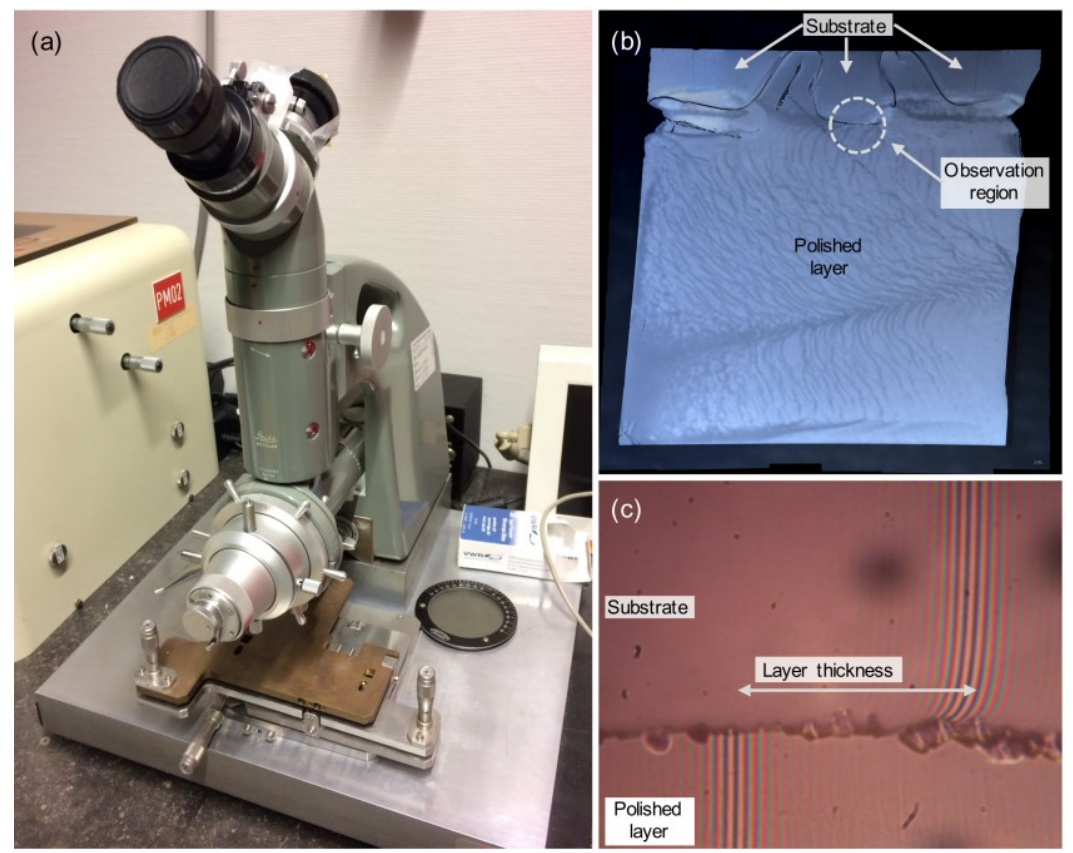

Figure 3.7 (a) Photograph of Leitz interference microscope used to monitor the thickness of the laser during polishing. (b) Example of a sample with polished epitaxial layer. The substrate was not fully dipped into the crucible during the growth process, hence the top section of the sample was not grown with epitaxial layer. The step-like interface circled in (b) was used to measure the thickness of the polished layer. The irregular curved pattern apparent on the sample was caused by unintentional growth deposits on the rear unpolished surface. (c) shows the observed interference fringes at the interface in between the substrate and the polished epitaxial layer. The fringes are shifted due to the step-height difference. 


\subsubsection{Material composition}

The $\mathrm{Yb}^{3+}$ concentrations of the samples were determined with an Energy Dispersive Xray module attached to a Scanning Electron Microscope (Zeiss Merlin HR-SEM) in MESA+ Institute for Nanotechnology, University of Twente. The surface of the samples was lapped, polished, and coated with a thin carbon layer prior to the characterization. The carbon layer prevents build-up of electrons on the sample. Strong charging effect had been observed during preliminary measurements prior to carbon layer coating as the $\mathrm{KRE}\left(\mathrm{WO}_{4}\right)_{2}$ was not electrically conductive. Therefore, spectrum acquisition could only be performed with source power $<10 \mathrm{keV}$. The carbon layer deposited on the sample eased the charging effect and allowed data collection using higher source power, resulting in lower uncertainty of composition analysis result.

Composition analysis was performed on two samples with nominal composition of $\mathrm{KGd}_{0.425} \mathrm{Yb}_{0.575}\left(\mathrm{WO}_{4}\right)_{2}$ [grown on $\mathrm{KY}\left(\mathrm{WO}_{4}\right)_{2}$ ] and $\mathrm{KLu}_{0.25} \mathrm{Yb}_{0.75}\left(\mathrm{WO}_{4}\right)_{2}$ [grown on $\mathrm{KLu}\left(\mathrm{WO}_{4}\right)_{2}$ ]. Example of scan spectra can be found in Figure 3.8. Based on multiple points measurements, the $\mathrm{Yb}^{3+}$ on these layers are found to be $0.57 \pm 0.03$ at.\% and $0.76 \pm 0.03$ at. $\%$, respectively, which are in agreement with their nominal compositions. These samples were used for spectroscopic and gain experiments and they will be referred as $\mathrm{KGd}_{0.43} \mathrm{Yb}_{0.57}\left(\mathrm{WO}_{4}\right)_{2}$ and $\mathrm{KLu}_{0.24} \mathrm{Yb}_{0.76}\left(\mathrm{WO}_{4}\right)_{2}$, respectively, hereafter.
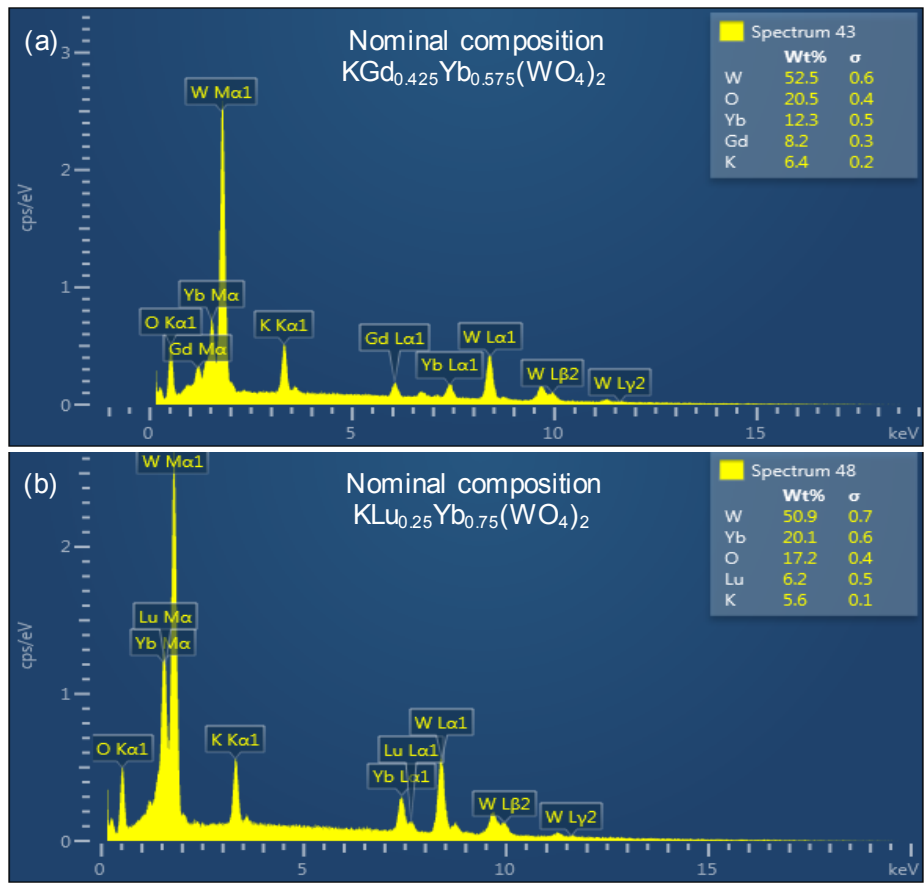

Figure 3.8 Composition analysis spectra of samples with nominal composition of (a) $\mathrm{KGd}_{0.425} \mathrm{Yb}_{0.575}\left(\mathrm{WO}_{4}\right)_{2}$, and (b) $\mathrm{KLu}_{0.25} \mathrm{Yb}_{0.75}\left(\mathrm{WO}_{4}\right)_{2}$. 


\subsubsection{Refractive index}

The refractive index of the epitaxial layers was measured with $m$-line spectroscopy (Metricon 2010M film prism coupler) using light sources at $632.8 \mathrm{~nm}, 830 \mathrm{~nm}$, $1300 \mathrm{~nm}$, and $1550 \mathrm{~nm}$ wavelengths. Refractive index measurements on $\mathrm{KY}_{1-x-y-z} \mathrm{Gd}_{x} \mathrm{Lu}_{y} \mathrm{Yb}_{z}\left(\mathrm{WO}_{4}\right)_{2}$ layers grown on $\mathrm{KY}\left(\mathrm{WO}_{4}\right)_{2}$ substrate with $\mathrm{Yb}^{3+}$ ranging from 2.5 at.\% to 47.5 at.\% were performed by Sergio A. Vázquez-Córdova and the results had been reported in [65].

Figure 3.9(a) shows the measurement results for the $\mathrm{KGd}_{0.43} \mathrm{Yb}_{0.57}\left(\mathrm{WO}_{4}\right)_{2}$ grown on $\mathrm{KY}\left(\mathrm{WO}_{4}\right)_{2}$ substrate. The measured values were used for curve fitting based on the Sellmeier equation with two coefficients [65]

$$
n^{2}=1+\frac{B \lambda^{2}}{\lambda^{2}-C^{2}}
$$

where $\lambda$ is wavelength in $\mu \mathrm{m}$ whereas $B$ and $C$ are coefficients obtained from leastsquare fitting. The fitted $B$ and $C$ are shown in Table 3.4. Based on the fitted curve in Figure 3.9(a), the refractive index at $932 \mathrm{~nm}$ and $981 \mathrm{~nm}$ wavelengths are found to be 2.0237 and 2.0213, respectively, at $N_{m}$. The corresponding refractive index contrast with respect to the substrate at $981 \mathrm{~nm}$ is $\sim 0.0123$.

Table 3.4 Fitted Sellmeier coefficients for $\mathrm{KGd}_{0.43} \mathrm{Yb}_{0.57}\left(\mathrm{WO}_{4}\right)_{2}$ grown on $\mathrm{KY}\left(\mathrm{WO}_{4}\right)_{2}$ substrate.

\begin{tabular}{|c|c|c|}
\hline Polarization & $\boldsymbol{B}$ (unitless) & $\boldsymbol{C}(\boldsymbol{\mu m})$ \\
\hline$N_{g}$ & 3.1619 & 0.1706 \\
\hline$N_{m}$ & 3.0006 & 0.1630 \\
\hline$N_{p}$ & 2.8785 & 0.1605 \\
\hline
\end{tabular}
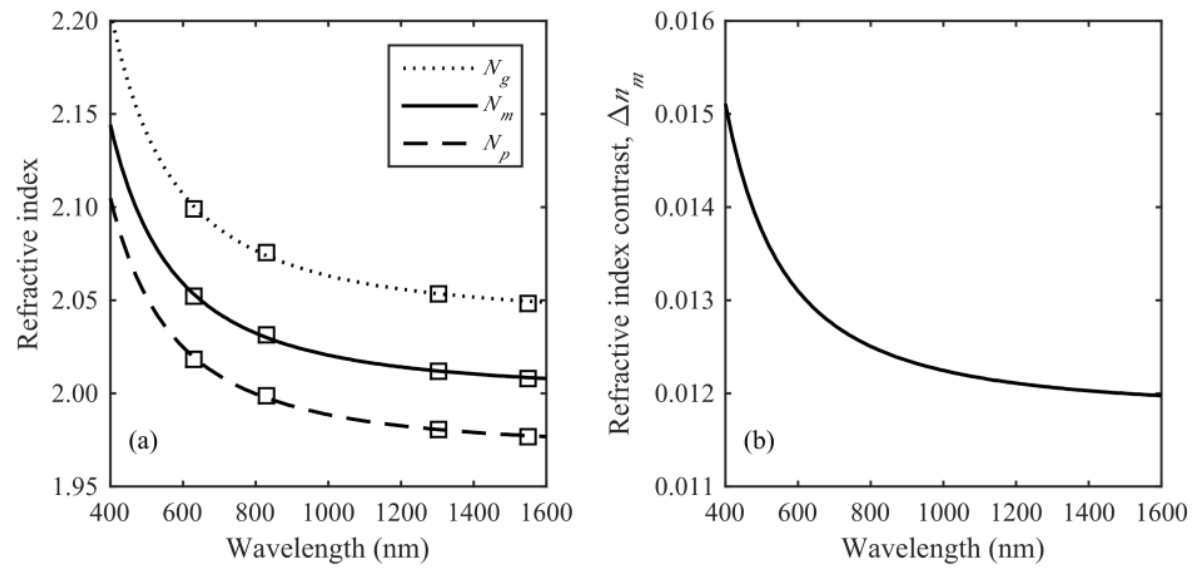

Figure 3.9. (a) Measured (squares) and fitted (lines) refractive indices of $\mathrm{KGd}_{0.43} \mathrm{Yb}_{0.57}\left(\mathrm{WO}_{4}\right)_{2}$. (b) Refractive index contrast of $\mathrm{KGd}_{0.43} \mathrm{Yb}_{0.57}\left(\mathrm{WO}_{4}\right)_{2}$ with respect to $\mathrm{KY}\left(\mathrm{WO}_{4}\right)_{2}$ substrate at $N_{m}$ axis. 


\subsection{Summary}

A thorough analysis on the properties of bulk crystals of $\mathrm{KRE}\left(\mathrm{WO}_{4}\right)_{2}$ has been shown in this chapter. The understanding of these properties allow the deduction of approaches used to grow high $\mathrm{Yb}^{3+}$ concentration epitaxial layers. Apart from that, the details of liquid phase epitaxy growth, lapping and polishing procedures, as well as composition and optical characterization methods have been explain.

Particularly, the crystallographic, optical, and thermal properties of bulk crystals of $\mathrm{KGd}\left(\mathrm{WO}_{4}\right)_{2}, \mathrm{KLu}\left(\mathrm{WO}_{4}\right)_{2}, \mathrm{KY}\left(\mathrm{WO}_{4}\right)_{2}$, and $\mathrm{KYb}\left(\mathrm{WO}_{4}\right)_{2}$ have been studied. The unit cells parameters of $\mathrm{KLu}\left(\mathrm{WO}_{4}\right)_{2}$ are close to those of $\mathrm{KYb}\left(\mathrm{WO}_{4}\right)_{2}$, hence using $\mathrm{KLu}\left(\mathrm{WO}_{4}\right)_{2}$ as substrate may permit the growth of high $\mathrm{Yb}^{3+}$ concentration epitaxial layer with minimal lattice mismatch and refractive index contrast in the order of $10^{-3}$ at $N_{m}$ polarization. Growing high $\mathrm{Yb}^{3+}$ concentration epitaxial layer on $\mathrm{KY}\left(\mathrm{WO}_{4}\right)_{2}$ substrate, on the other hand, would maximize the refractive index contrast which is beneficial for waveguiding purpose.

Taking into consideration the properties of the double tungstates, two approaches were used to prepare high $\mathrm{Yb}^{3+}$ concentration epitaxial layer used in this thesis. The first one used a lattice engineering method to incorporate high amount of $\mathrm{Yb}^{3+}$ on epitaxial layer grown on $\mathrm{KY}\left(\mathrm{WO}_{4}\right)_{2}$ by including optically inert $\mathrm{Gd}^{3+}$ and $\mathrm{Lu}^{3+}$ in the layer. Using this approach, the highest possible $\mathrm{Yb}^{3+}$ content was realized on epitaxial layers with a composition of $\mathrm{KGd}_{0.43} \mathrm{Yb}_{0.57}\left(\mathrm{WO}_{4}\right)_{2}$, in which the $\mathrm{Y}^{3+}$ sites were fully replaced by $\mathrm{Gd}^{3+}$ and $\mathrm{Yb}^{3+}$. The refractive index contrast obtained from such layer is $\sim 0.012$ with respect to the substrate at $981 \mathrm{~nm}$ wavelength for $N_{m}$ polarization. The second approach leveraged on the favorable unit cells parameters of $\mathrm{KLu}\left(\mathrm{WO}_{4}\right)_{2}$ to grow epitaxial layer by direct substitution of $\mathrm{Lu}^{3+}$ with $\mathrm{Yb}^{3+}$. Sample grown with this approach had been supplied by Universitat Rovira i Virgili (URV), Tarragona and its composition was found to be $\mathrm{KLu}_{0.24} \mathrm{Yb}_{0.76}\left(\mathrm{WO}_{4}\right)_{2}$. 



\section{Chapter 4}

\section{Spectroscopic properties}

\subsection{Overview}

The luminescence lifetime, absorption cross-section, and emission cross-section are three fundamental spectroscopic properties for optically active media. The spectroscopic properties of potassium rare-earth double tungstates doped with trivalent ytterbium, $\mathrm{KRE}\left(\mathrm{WO}_{4}\right)_{2}: \mathrm{Yb}^{3+}$, were reported as early as 1997 by Kuleshov et al. [71, 123]. Thereafter, numerous studies were performed on bulk crystals of $\mathrm{KGd}\left(\mathrm{WO}_{4}\right)_{2}: \mathrm{Yb}^{3+}[71,73], \mathrm{KLu}\left(\mathrm{WO}_{4}\right)_{2}: \mathrm{Yb}^{3+}$ [74, 107], $\mathrm{KY}\left(\mathrm{WO}_{4}\right)_{2}: \mathrm{Yb}^{3+}$ [71] and $\mathrm{KYb}\left(\mathrm{WO}_{4}\right)_{2}[104,106]$. On the other hand, comprehensive spectroscopy studies on $\mathrm{KRE}\left(\mathrm{WO}_{4}\right)_{2}: \mathrm{Yb}^{3+}$ epitaxial layers $[107,124]$ are relatively scarce.

Determination of luminescence lifetime in $\mathrm{Yb}^{3+}$-doped gain material is complicated by the radiation trapping phenomenon which causes considerable lengthening of measured lifetime [93]. As a result, lifetime values ranging from $\sim 200 \mu \mathrm{s}$ to $850 \mu \mathrm{s}$ has been reported in $\mathrm{KRE}\left(\mathrm{WO}_{4}\right)_{2}: \mathrm{Yb}^{3+}[71,72,82,92,123,125$, 126]. On the other hand, reported peak absorption cross-section of $\mathrm{Yb}^{3+}$ in $\mathrm{KRE}\left(\mathrm{WO}_{4}\right)_{2}$ at $\sim 981 \mathrm{~nm}$ wavelength found in the literature varies widely from $7.1 \times 10^{-20} \mathrm{~cm}^{2}$ to $13.3 \times 10^{-20} \mathrm{~cm}^{2}[71,127]$. Apart from these discrepancies, it is also unclear whether the luminescence lifetime and transition cross-sections in $\mathrm{KRE}\left(\mathrm{WO}_{4}\right)_{2}: \mathrm{Yb}^{3+}$ epitaxial layers with $>50$ at. $\% \mathrm{Yb}^{3+}$ remain the same given substantial amount of $\mathrm{Yb}^{3+}$ ions in these samples. Furthermore, as highly $\mathrm{Yb}^{3+}$-doped devices may operate at elevated temperatures, knowledge of the dependence of spectroscopic properties on temperature is necessary to understand and to model the operational characteristics of the device. However, the temperature dependence of luminescence lifetime and transition crosssections in $\mathrm{KRE}\left(\mathrm{WO}_{4}\right)_{2}: \mathrm{Yb}^{3+}$ has not been reported prior to this work.

Luminescence lifetime, transition cross-sections, and their temperature dependence in high $\mathrm{Yb}^{3+}$ concentration epitaxial layers will be presented in subsequent sections. The radiation trapping effect which severely affects the accuracy of measured lifetime in $\mathrm{KRE}\left(\mathrm{WO}_{4}\right)_{2}: \mathrm{Yb}^{3+}$ media is discussed and a confocal lifetime measurement setup proposed to mitigatie such effect is introduced. Lifetime measurements on epitaxial layers with $\mathrm{Yb}^{3+}$ ranging from 1.2 at.\% to 76 at.\% are performed to study the concentration dependent lifetime quenching. Subsequently, the transition cross-sections 
in epitaxial layers with 57 at. $\%$ and 76 at. $\% \mathrm{Yb}^{3+}$ are investigated. The cross-sections of these composite layers are compared to the experimental results of bulk $\mathrm{KRE}\left(\mathrm{WO}_{4}\right)_{2}: \mathrm{Yb}^{3+}$ crystals. Next, the temperature dependence of lifetime and transition cross-sections for the sample with 57 at. $\% \mathrm{Yb}^{3+}$ is examined. The chapter is then ended with a summary section.

\subsection{Luminescent lifetime}

This section begins with a brief discussion on the radiation trapping effect. Next, a novel confocal lifetime measurement setup which is proposed to mitigate the radiation trapping effect will be introduced. Subsequently, concentration dependence and power dependence of luminescence lifetime in $\mathrm{KRE}\left(\mathrm{WO}_{4}\right)_{2}: \mathrm{Yb}^{3+}$ epitaxial layers will be presented and discussed.

\subsubsection{Radiation trapping}

Measurement of luminescent lifetime in a rare-earth-activated gain medium with strong spectral overlap of luminescence and ground-state absorption is known to be severely affected by radiation trapping effect [93]. The radiation trapping effect, which is also known as reabsorption effect, is illustrated in Figure 4.1. A photon spontaneously emitted by an excited ion can be absorbed by another, potentially even rather distant ion in its ground state, which in turn emits a new photon, resulting in considerable lengthening of measured luminescence lifetime. Radiation trapping is particularly prominent for the ${ }^{2} \mathrm{~F}_{5 / 2} \rightarrow{ }^{2} \mathrm{~F}_{7 / 2}$ transition in $\mathrm{Yb}^{3+}$ at $\sim 1 \mu \mathrm{m}$ wavelength and is also apparent for transitions such as the ${ }^{4} \mathrm{I}_{13 / 2} \rightarrow{ }^{4} \mathrm{I}_{15 / 2}$ in $\mathrm{Er}^{3+}$ at $\sim 1.5 \mu \mathrm{m}$, the ${ }^{3} \mathrm{~F}_{4} \rightarrow{ }^{3} \mathrm{H}_{6}$ in $\mathrm{Tm}^{3+}$ at $\sim 2 \mu \mathrm{m}$, and the ${ }^{5} \mathrm{I}_{7} \rightarrow{ }^{5} \mathrm{I}_{8}$ in $\mathrm{Ho}^{3+}$ at $\sim 2.1 \mu \mathrm{m}[27,93]$.

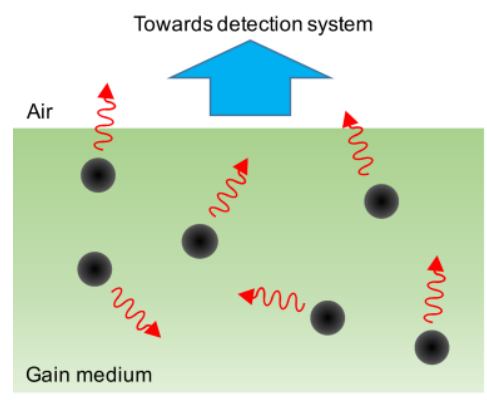

(a) Without radiation trapping

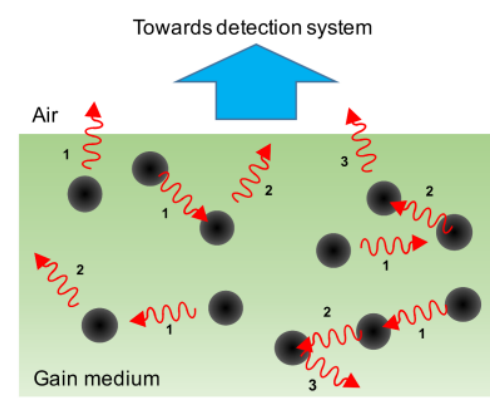

(b) With radiation trapping

Figure 4.1 Illustration of luminescence lifetime detection under the situations of (a) ideal case where spontaneously emitted photons reach the detection system without radiation trapping, and (b) severe radiation trapping where a large number of originally emitted photons are reabsorbed and new photons are emitted, resulting in elongation of measured lifetime. 
In the absence of strong lifetime quenching effect, as in the case of $\mathrm{KRE}\left(\mathrm{WO}_{4}\right)_{2}: \mathrm{Yb}^{3+}[83]$, a higher concentration of active ions in the material increases the occurrence of the reabsorption process and leads to the observation of longer measured lifetimes [92]. Contrarily, with the presence of strong quenching, the lifetime initially increases with the increasing active ions concentration, after which it sharply decreases with the further increase of active ion concentration [27, 83]. The lifetime quenching in $\mathrm{Yb}^{3+}$-doped media is largely caused by trace impurities, such as $\mathrm{Tm}^{3+}$, $\mathrm{Er}^{3+}$, or $\mathrm{Cr}^{3+}$ [93]. In certain media, such as $\mathrm{YAG}: \mathrm{Yb}^{3+}$, the presence of $\mathrm{Yb}^{2+}$ in the asgrown crystals also lead to severe lifetime quenching [83, 128].

Several methods have been suggested to minimize the impact of radiation trapping. Sandwiching a thin active sample by two undoped slabs to avoid total internal reflections, along with aperturing of the emission, was demonstrated on $\mathrm{YAG} \mathrm{Yb}^{3+}$ [93] and $\mathrm{LiNbO}_{3}: \mathrm{Er}^{3+}$ [129]. Measurements on powdered samples diluted in liquid were used to determine the lifetime in $\mathrm{YVO}_{4}: \mathrm{Yb}^{3+}[130], \mathrm{YAlO}_{3}: \mathrm{Yb}^{3+}[131], \mathrm{KY}\left(\mathrm{WO}_{4}\right)_{2}: \mathrm{Yb}^{3+}$ [82], and $\mathrm{KGd}\left(\mathrm{WO}_{4}\right)_{2}: \mathrm{Yb}^{3+}$ [126]. This approach requires extensive sample preparation which is destructive in nature. The pinhole method was suggested by Kühn et al. as a non-destructive way of quantifying the lifetime by use of pinholes with diameters ranging from 0.35 to $2.5 \mathrm{~mm}$ [132]. The pinhole method has been tested on various $\mathrm{Yb}^{3+}$-doped hosts $[107,132,133]$. It involves multiple measurements of the luminescence lifetime collected through pinholes of decreasing diameter. The lifetime value extrapolated to zero pinhole diameter represents the intrinsic luminescence lifetime. The method, however, may suffer from an uncertainty of the lifetime value due to fluctuations of the measured data and the extrapolation procedure.

In order to largely suppress radiation trapping, the detection volume should be matched to the pumped volume, and this volume should be as small as possible [134]. This goal can be achieved by means of a confocal setup [135]. The use of a confocal setup for lifetime measurements on a rare-earth-ion-doped material was reported by Brynolfsson [136]. However, his implementation was essentially a modified version of the pinhole method proposed by Kühn et al., to the extent that multiple measurements using pinholes with diameters ranging from $1 \mathrm{~mm}$ to $10 \mathrm{~mm}$ were performed, followed by an extrapolation procedure.

A confocal luminescent lifetime measurement setup is proposed in the following sub-section to mitigate the influence of radiation trapping on the measured lifetime. The non-destructive measurement method allows one to directly extract the lifetime from the measured decay curves without performing multiple measurements with different pinholes and subsequent data extrapolation. This procedure is used to study the lifetime quenching in highly-doped $\mathrm{KRE}\left(\mathrm{WO}_{4}\right)_{2}: \mathrm{Yb}^{3+}$ crystalline layers. 


\subsubsection{Lifetime measurement using confocal method}

Figure 4.2 shows the proposed setup for confocal luminescence lifetime measurements. The pump beam is focused perpendicularly onto the epitaxial layer under investigation. The luminescence is collected, spatially filtered using a pinhole, and relayed to the detection system. A flip mirror and a camera are used to assist in positioning the sample. The limitation of the confocal method is due, firstly, to the pump spot size, which should ideally be infinitesimally small, and, secondly, the effective discrimination of luminescence from the non-confocal region via spatial filtering. The luminescence emissions originated from out-of-focus planes are blocked by the pinhole. Naturally, the rejection rate of out-of-focus emissions is higher when the pinhole is smaller, but this also results in weaker detected intensity.

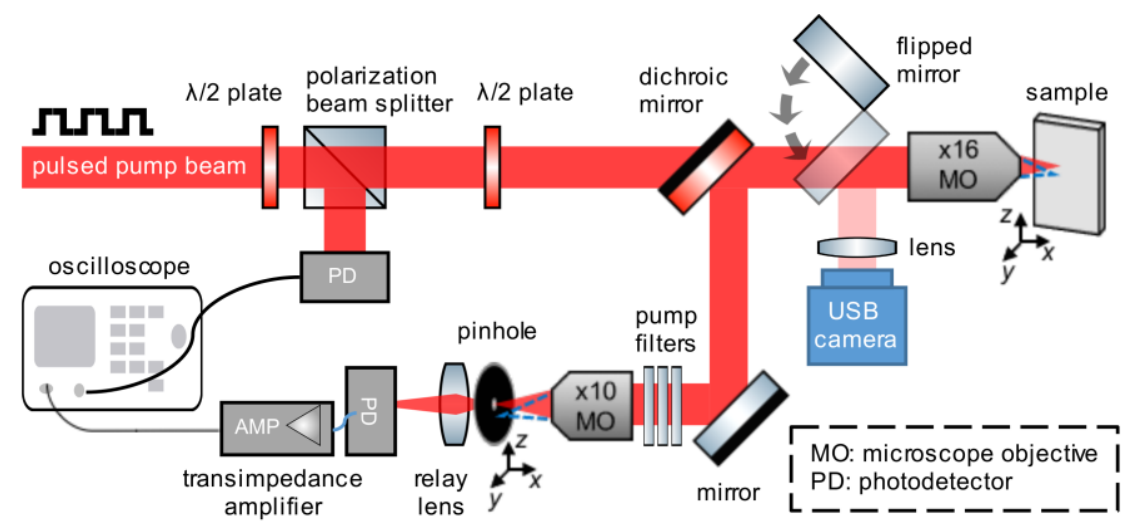

Figure 4.2 Schematic diagram of the confocal luminescence lifetime measurement setup. The blue dashed foci in front of the microscope objectives illustrate the discrimination of undesirable reemitted photons coming out of the unpumped region of the sample from the detection system by the use of pinhole.

The pump configuration implemented in the experiment consists of a fiberpigtailed single-mode diode laser operating at $981 \mathrm{~nm}$ wavelength and modulated at $233 \mathrm{~Hz}$ with $50 \%$ duty cycle, whose emitted light passes through an optical isolator, after which it is collimated and expanded in free space. The repetition rate and the duty cycle of the pump beam are chosen such that the unpumped duration is more than six times the measured lifetime, allowing for complete relaxation of the $\mathrm{Yb}^{3+}$ ions between two excitations.

A half-wave-plate $(\lambda / 2$ plate) and a polarizing beam splitter (PBS) are used to control the power incident upon the sample, as shown in Figure 4.2. A silicon ( $\mathrm{Si}$ ) photodetector (Thorlabs DET10A) is placed into the reflected arm of the PBS to serve as a triggering source. An additional $\lambda / 2$ plate is placed after the PBS to align the polarization near to the E ||$N_{m}$ optical axis of the sample for maximum pump absorption. 
The pump beam then passes through a dichroic mirror (Thorlabs DSMP1000) and is focused by a $\times 16$ microscope objective $(\mathrm{MO})(\mathrm{NA}=0.32)$ to a spot size of $\sim 7 \mu \mathrm{m}$.

The luminescence from the sample is collected by the same MO, reflected by the dichroic mirror, and then focused by a $\times 10 \mathrm{MO}(\mathrm{NA}=0.25)$ through a pinhole with $10 \mu \mathrm{m}$ diameter. The luminescence passing through the pinhole is then relayed to a second Si photodetector using a $30 \mathrm{~mm}$ lens. The collected signal is amplified (FEMTO DHPCA-100), sent to a digital storage oscilloscope (Agilent Infinium 54845A), and averaged for 4096 times. Detection of the back-reflected pump beam indicates that the temporal resolution of the measurement system is limited to $\sim 6 \mu \mathrm{s}$ by the switching speed of the pump laser. During the experiment, three pump filters (Thorlabs FEL1000) are placed before the $\times 10 \mathrm{MO}$. These filters along with the dichroic mirror strongly attenuate the back-reflected pump light by $\sim 100 \mathrm{~dB}$.

The proposed lifetime measurement method is applied to $\mathrm{KRE}\left(\mathrm{WO}_{4}\right)_{2}: \mathrm{Yb}^{3+}$ epitaxial layers with $\mathrm{Yb}^{3+}$ concentrations ranging from 1.2 at. $\%$ up to 76 at. $\%\left(\sim 8 \times 10^{19}\right.$ $-5 \times 10^{21} \mathrm{~cm}^{-3}$ ) for a systematic study on the potential lifetime quenching. The samples include $\mathrm{KRE}\left(\mathrm{WO}_{4}\right)_{2}: \mathrm{Yb}^{3+}$ epitaxial layers, with $\mathrm{Yb}^{3+}$ concentrations varying from 1.2 at. $\%$ to 57 at.\%, grown onto undoped potassium yttrium double tungstate, $\mathrm{KY}\left(\mathrm{WO}_{4}\right)_{2}$, substrates. The highest-doped sample is a $\mathrm{KLu}\left(\mathrm{WO}_{4}\right)_{2}: \mathrm{Yb}^{3+}(76$ at.\%) grown onto an undoped $\mathrm{KLu}\left(\mathrm{WO}_{4}\right)_{2}$ substrate provided by Universitat Rovira i Virgili, Spain. An overview of the investigated samples is given in Table 4.1. The samples with 47.5 at.\%, 53.5 at.\%, 57 at.\%, and 76 at.\% $\mathrm{Yb}^{3+}$ are lapped and polished parallel to the substrate. The other samples are not polished. The sample with 47.5 at. $\% \mathrm{Yb}^{3+}$ is overgrown with undoped $\mathrm{KY}\left(\mathrm{WO}_{4}\right)_{2}$.

Table 4.1 Material composition and layer thickness of the $\mathbf{Y b}^{3+}$-doped potassium rare-earth double tungstate epitaxial films used for lifetime measurements.

\begin{tabular}{|c|c|c|}
\hline Nominal composition of epitaxial layer & Substrate & Layer thickness $(\boldsymbol{\mu m})$ \\
\hline $\mathrm{KY}_{0.988} \mathrm{Yb}_{0.012}\left(\mathrm{WO}_{4}\right)_{2}$ & & 36 \\
$\mathrm{KY}_{0.75} \mathrm{Gd}_{0.10} \mathrm{Yb}_{0.15}\left(\mathrm{WO}_{4}\right)_{2}$ & & 20 \\
$\mathrm{KY}_{0.67} \mathrm{Gd}_{0.13} \mathrm{Yb}_{0.20}\left(\mathrm{WO}_{4}\right)_{2}$ & & 42 \\
$\mathrm{KGd}_{0.462} \mathrm{Lu}_{0.213} \mathrm{Yb}_{0.325}\left(\mathrm{WO}_{4}\right)_{2}$ & $\mathrm{KY}\left(\mathrm{WO}_{4}\right)_{2}$ & 20 \\
$\mathrm{KGd}_{0.447} \mathrm{Lu}_{0.078} \mathrm{Yb}_{0.475}\left(\mathrm{WO}_{4}\right)_{2}$ & & 6.7 \\
$\mathrm{KGd}_{0.44} \mathrm{Lu}_{0.025} \mathrm{Yb}_{0.535}\left(\mathrm{WO}_{4}\right)_{2}$ & & 6 \\
$\mathrm{KGd}_{0.43} \mathrm{Yb}_{0.57}\left(\mathrm{WO}_{4}\right)_{2}$ & & 32 \\
\hline $\mathrm{KLu}_{0.24} \mathrm{Yb}_{0.76}\left(\mathrm{WO}_{4}\right)_{2}$ & $\mathrm{KLu}\left(\mathrm{WO}_{4}\right)_{2}$ & 124 \\
\hline
\end{tabular}




\subsubsection{Concentration dependence of luminescence lifetime}

The dependence of luminescence lifetime on the concentration of $\mathrm{Yb}^{3+}$ is investigated using low launched pump power which is $<2 \mathrm{~mW}$ unless specified otherwise to avoid detection of non-exponential decay component, which will be further examined in Section 4.2.4, and to minimize potential pump-induced heating at the focal spot. Figure 4.3 depicts the representative measured luminescence decay curves for samples with 1.2 at.\%, 57 at.\%, and 76 at.\% $\mathrm{Yb}^{3+}$, respectively. Evidently, the measured decay curves exhibit a single exponential decay regardless of the $\mathrm{Yb}^{3+}$ concentration. In the case of the sample with 1.2 at.\% $\mathrm{Yb}^{3+}$, the launched pump power was increased to $\sim 50 \mathrm{~mW}$ to obtain significant luminescence. In all cases, the lifetime is obtained by fitting the time-dependent luminescence intensity by

$$
I(t)=I_{0} \exp (-t / \tau)+I_{\text {base }},
$$

where $I_{0}$ is the initial luminescence intensity upon cut-off of the pump $(t=0), \tau$ is the fitted lifetime, and $I_{b a s e}$ represents the baseline noise level of the detected signal.
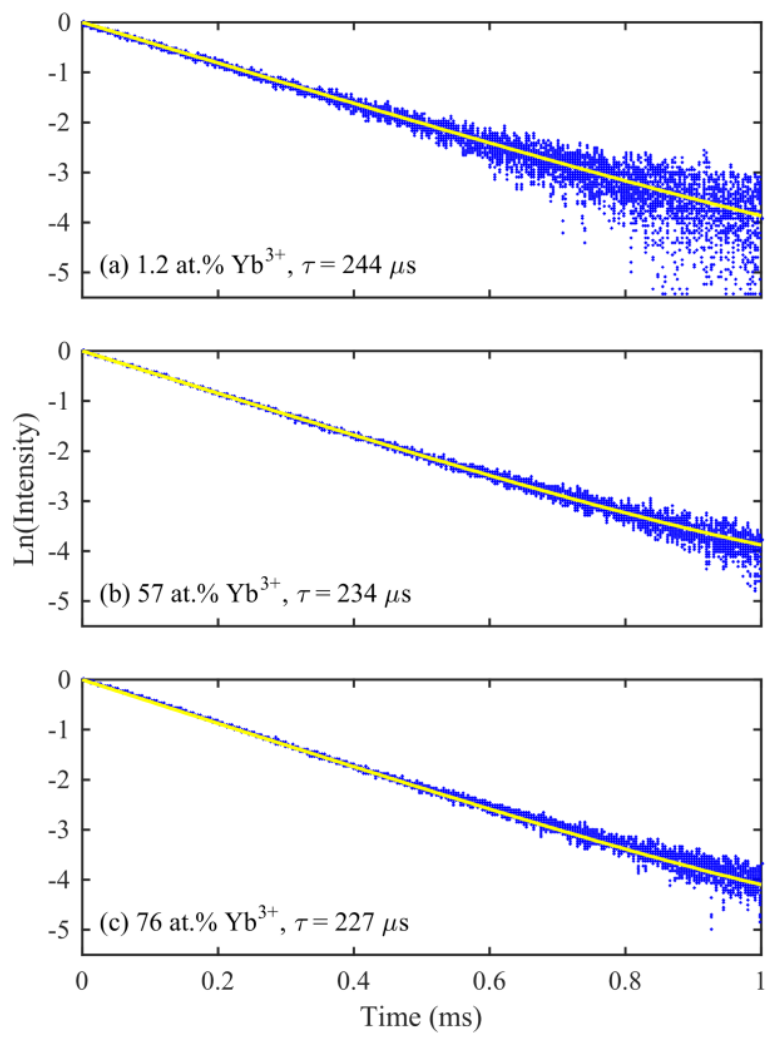

Figure 4.3 Measured (blue) and fitted (yellow) luminescent decay curves of samples with (a) 1.2 at.\%, (b) 57 at.\%, and (c) 76 at.\% of $\mathrm{Yb}^{3+}$. 
The resulting luminescence lifetime values versus $\mathrm{Yb}^{3+}$ concentration are shown in Figure 4.4 as blue inverted triangles. For each composition, measurements on several randomly chosen positions on the sample were carried out and the resulting lifetime values were averaged. For example, lifetime values of $245 \pm 3 \mu \mathrm{s}, 228 \pm 10 \mu \mathrm{s}$, and $222 \pm 9 \mu$ s were measured for samples with $\mathrm{Yb}^{3+}$ concentrations of 1.2 at. $\%, 57$ at.\%, and 76 at. $\%$ respectively, which are comparable to the lifetime values in $\mathrm{KRE}\left(\mathrm{WO}_{4}\right)_{2}: \mathrm{Yb}^{3+}$ bulk crystals [83] and thin films [107] extrapolated by the pinhole method (green squares in Figure 4.4) and to the lifetime values measured in bulk $\mathrm{KRE}\left(\mathrm{WO}_{4}\right)_{2}: \mathrm{Yb}^{3+}$ ground to powder and diluted in liquid ethylene glycol (red circles in Figure 4.4) $[82,104,126]$. For comparison, measurements were performed by removing the $10 \mu \mathrm{m}$ pinhole in the setup, i.e., in non-confocal mode. The resulting luminescence lifetimes in non-confocal mode (black triangles in Figure 4.4) are elongated to $256 \mu \mathrm{s}$ for the sample with 1.2 at. $\% \mathrm{Yb}^{3+}$ and $325 \mu$ s for the sample with 76 at. $\% \mathrm{Yb}^{3+}$, clearly demonstrating the effect of radiation trapping. The setup described in this work permits direct measurement of luminescence lifetimes thanks to the small probe volume, in combination with confocal detection, in which the $10 \mu \mathrm{m}$ pinhole rejects the luminescence trapped and reemitted from outside the focal volume. Reported lifetime values measured on $\mathrm{KRE}\left(\mathrm{WO}_{4}\right)_{2}: \mathrm{Yb}^{3+}$ with low $\mathrm{Yb}^{3+}$ concentration without using the pinhole method nor a powdered sample vary widely from 300 to $850 \mu$ s [71, 74, 92, $123,125]$. A detailed comparison of the lifetime values measured using the confocal method against the reported values available in the literature can be found in Table 4.2.

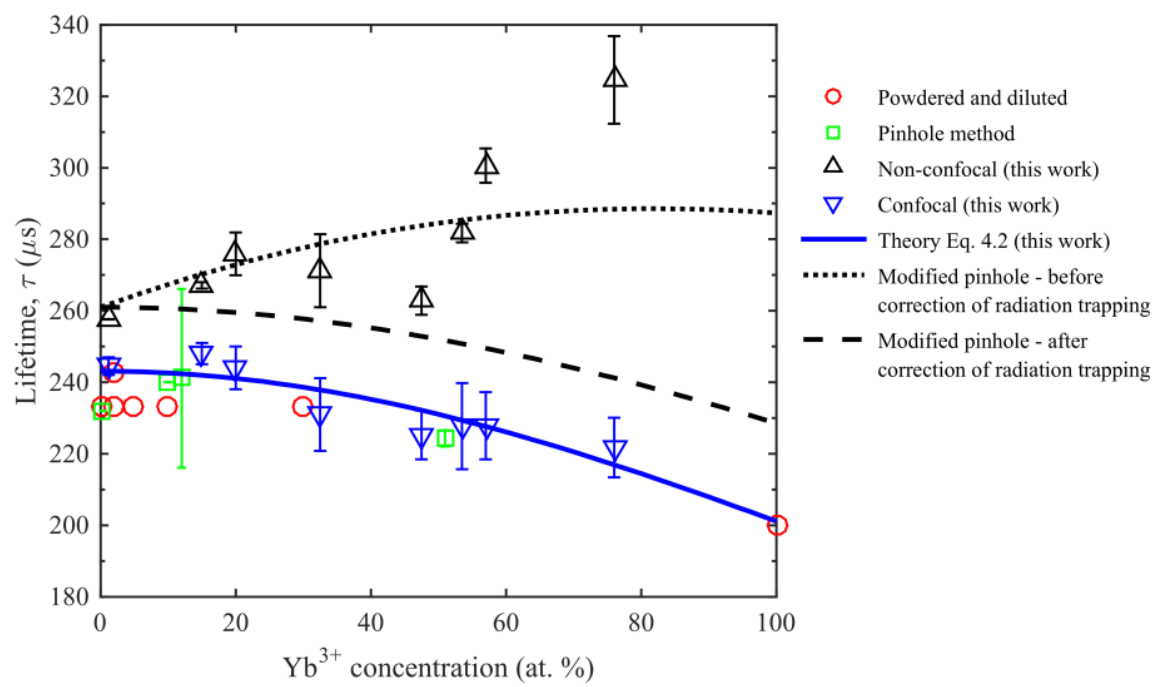

Figure 4.4 Luminescence lifetimes measured with (blue inverted triangles) and without (black triangles) confocal feature. Lifetime values measured using powdered bulk crystals of $\mathrm{KRE}\left(\mathrm{WO}_{4}\right)_{2}: \mathrm{Yb}^{3+}[82,104,126]$ (red circles), pinhole method on $\mathrm{KRE}\left(\mathrm{WO}_{4}\right)_{2}$ : $^{\mathrm{Yb}^{3+}}$ crystals $[83]$ and thin films [107] (green squares), and modified pinhole method before (dotted line) and after (dashed line) correction of radiation trapping [65] are shown for comparison. The blue line is the fitted curve based on Eq. 4.2. 
Table 4.2 Comparison of the lifetime values measured using the confocal method proposed in this work to the reported lifetime values in the literature.

\begin{tabular}{|c|c|c|c|}
\hline $\begin{array}{l}\text { Measurement } \\
\text { technique }\end{array}$ & $\begin{array}{c}\text { Host crystal / } \\
\text { Composition of epitaxial layer }\end{array}$ & $\begin{array}{c}\text { Concentration of } \\
\mathbf{Y b}^{3+}(\text { at. } \%)\end{array}$ & $\begin{array}{l}\text { Lifetime } \\
\quad(\mu s)\end{array}$ \\
\hline \multirow{8}{*}{$\begin{array}{l}\text { Confocal method } \\
\text { (this work) }\end{array}$} & $\mathrm{KY}_{0.988} \mathrm{Yb}_{0.012}\left(\mathrm{WO}_{4}\right)_{2}$ epitaxial layer & 1.2 & $245 \pm 3$ \\
\hline & $\mathrm{KY}_{0.75} \mathrm{Gd}_{0.10} \mathrm{Yb}_{0.15}\left(\mathrm{WO}_{4}\right)_{2}$ epitaxial layer & 15 & $248 \pm 3$ \\
\hline & $\mathrm{KY}_{0.67} \mathrm{Gd}_{0.13} \mathrm{Yb}_{0.20}\left(\mathrm{WO}_{4}\right)_{2}$ epitaxial layer & 20 & $244 \pm 6$ \\
\hline & $\mathrm{KGd}_{0.462} \mathrm{Lu}_{0.213} \mathrm{Yb}_{0.325}\left(\mathrm{WO}_{4}\right)_{2}$ epitaxial layer & 32.5 & $231 \pm 11$ \\
\hline & $\mathrm{KGd}_{0.447} \mathrm{Lu}_{0.078} \mathrm{Yb}_{0.475}\left(\mathrm{WO}_{4}\right)_{2}$ epitaxial layer & 47.5 & $225 \pm 7$ \\
\hline & $\mathrm{KGd}_{0.44} \mathrm{Lu}_{0.025} \mathrm{Yb}_{0.535}\left(\mathrm{WO}_{4}\right)_{2}$ epitaxial layer & 53.5 & $228 \pm 12$ \\
\hline & $\mathrm{KGd}_{0.43} \mathrm{Yb}_{0.57}\left(\mathrm{WO}_{4}\right)_{2}$ epitaxial layer & 57 & $228 \pm 10$ \\
\hline & $\mathrm{KLu}_{0.24} \mathrm{Yb}_{0.76}\left(\mathrm{WO}_{4}\right)_{2}$ epitaxial layer & 76 & $222 \pm 9$ \\
\hline \multirow[t]{5}{*}{ Pinhole method } & $\mathrm{KY}\left(\mathrm{WO}_{4}\right)_{2}$ crystal $[83]$ & 0.2 & 232 \\
\hline & $\mathrm{KY}\left(\mathrm{WO}_{4}\right)_{2}$ crystal $^{(\mathrm{i})}[136]$ & 5 & 257 \\
\hline & $\mathrm{KY}\left(\mathrm{WO}_{4}\right)_{2}$ crystal $[83]$ & 10 & 240 \\
\hline & $\mathrm{KLu}\left(\mathrm{WO}_{4}\right)_{2}: \mathrm{Yb}^{3+}$ epitaxial layer [107] & 12 & $241.1 \pm 25$ \\
\hline & $\mathrm{KLu}\left(\mathrm{WO}_{4}\right)_{2}: \mathrm{Yb}^{3+}$ epitaxial layer [107] & 52 & $224.3 \pm 2.3$ \\
\hline \multirow[t]{4}{*}{ Powder \& diluted } & $\mathrm{KY}\left(\mathrm{WO}_{4}\right)_{2}$ crystal [82] & $0.2,5,10$, and 30 & 233 \\
\hline & $\mathrm{KGd}\left(\mathrm{WO}_{4}\right)_{2}$ crystal $[114]$ & $\sim 1.5$ to $\sim 8$ & $275 \pm 5^{\text {(ii) }}$ \\
\hline & $\mathrm{KGd}\left(\mathrm{WO}_{4}\right)_{2}$ crystal [126] & 2 & 243 \\
\hline & $\mathrm{KYb}\left(\mathrm{WO}_{4}\right)_{2}$ crystal [104] & 100 & 200 \\
\hline \multirow[t]{5}{*}{ Others } & $\mathrm{KLu}\left(\mathrm{WO}_{4}\right)_{2}$ crystal [74] & 0.5 & 375 \\
\hline & $\mathrm{KY}\left(\mathrm{WO}_{4}\right)_{2}$ crystals $[92]$ & $1,5,10$, and 20 & $\geq 300$ \\
\hline & $\mathrm{KY}\left(\mathrm{WO}_{4}\right)_{2}$ and $\mathrm{KGd}\left(\mathrm{WO}_{4}\right)_{2}$ crystals [125] & 5 & $438-440$ \\
\hline & $\mathrm{KY}\left(\mathrm{WO}_{4}\right)_{2}$ and $\mathrm{KGd}\left(\mathrm{WO}_{4}\right)_{2}$ crystals [71] & $\sim 5$ & 600 \\
\hline & $\mathrm{KY}\left(\mathrm{WO}_{4}\right)_{2}$ and $\mathrm{KGd}\left(\mathrm{WO}_{4}\right)_{2}$ crystals [123] & 5 & 850 \\
\hline
\end{tabular}

(i) The author claimed that the measurement was performed using a confocal setup but the implementation resembles the pinhole method. This data is excluded from Figure 4.4.

(ii) The reported lifetime is noticeable higher than the others, possibly because the samples were only grounded but not diluted with index matching liquid. This data is excluded from Figure 4.4.

The reduction of lifetime with increasing $\mathrm{Yb}^{3+}$ concentration observed in Figure 4.4 is ascribed to concentration quenching due to non-radiative energy migration and subsequent energy transfer to a trap, e.g. a crystal defect or an impurity ion, which follows the phenomenological expression $[27,137]$

$$
\tau(N)=\frac{\tau_{0}}{1+(9 / 2 \pi)\left(N / N_{0}\right)^{2}},
$$

where $\tau_{0}$ is the measured lifetime at low $\mathrm{Yb}^{3+}$ concentration, $N$ is the $\mathrm{Yb}^{3+}$ concentration, and $N_{0}$ is the critical concentration beyond which the lifetime quenching is severe. Applying Eq. 4.2 to the measured data using the confocal setup shown in Figure 4.4, the fitted values of $\tau_{0}=(243.2 \pm 8) \mu$ s and $N_{0}=(1.69 \pm 0.6) \times 10^{22} \mathrm{~cm}^{-3}$ are 
obtained. The obtained $N_{0}$ value agrees reasonably well with our previous results [65] of $\tau_{0}=261 \mu \mathrm{s}$ and $N_{0}=2 \times 10^{22} \mathrm{~cm}^{-3}$ measured with the modified pinhole method on the similar set of samples. The difference in $\tau_{0}$ leads to the small offset between the blue and the dashed black curve in Figure 4.4. The high $N_{0}$ value which is much greater than the $\mathrm{Yb}^{3+}$ concentration of $6.4 \times 10^{21} \mathrm{~cm}^{-3}$ for $\mathrm{KYb}\left(\mathrm{WO}_{4}\right)_{2}$ [22], i.e. 100 at. $\% \mathrm{Yb}^{3+}$, signifies that the luminescence lifetime quenching due to increasing $\mathrm{Yb}^{3+}$ concentration is rather weak over the entire doping range. The lifetime value extrapolated to 100 at.\% $\mathrm{Yb}^{3+}$ doping is $201 \mu \mathrm{s}$, or $\sim 83 \%$ of the lifetime at low $\mathrm{Yb}^{3+}$ concentration. The results presented in this work reaffirm our previous finding [65] that the lifetime quenching is rather weak and $\mathrm{KRE}\left(\mathrm{WO}_{4}\right)_{2}$ with high $\mathrm{Yb}^{3+}$ concentration may be utilized for amplifiers and lasers.

\subsubsection{Power dependence of luminescence lifetime}

The use of confocal luminescence lifetime measurement setup allows the investigation of lifetime behavior under high excitation density which is similar to the operating condition of the amplifier. Luminescence lifetime decay curves measured with different launched pump power $P_{l}$ values are collected from sample with 57 at.\% $\mathrm{Yb}^{3+}$, i.e. $\mathrm{KGd}_{0.43} \mathrm{Yb}_{0.57}\left(\mathrm{WO}_{4}\right)_{2}$, and the results are plotted in Figure 4.5. At low $P_{l}$ of $1.44 \mathrm{~mW}$, the time-dependent luminescence exhibits single exponential decay. Nevertheless, with the increase of $P_{l}$, the detected decay curves exhibit non-exponential decay. At the highest $P_{l}$ of $29.17 \mathrm{~mW}$, an initial fast decay upon cut-off of pump power is observed. The non-exponential decay behavior indicates that energy transfer upconversion (ETU) process is present, in which the interaction of excited active ions within close distance leads to de-excitation of active ions from the excited state.

The decay curves are further analyzed by considering the rate equation model by Grant [138]. Grant's model assumes an infinitely fast energy-migration rate, leading to a "sea" of excitations in the metastable excited state, which are smeared out over the excitation volume. The non-linear nature of the ETU process is taken into account in the rate equation using a term $2 W_{E T U} N_{1}^{2}$, where $W_{E T U}$ is a coefficient with units of $\mathrm{cm}^{3} / \mathrm{s}, N_{1}$ is time dependent population density at the excited state, and the factor of 2 accounts for the interaction which involves two ions. Consequently, the rate of change of the population density at the excited state can be modeled with [139]

$$
\frac{d N_{1}(t)}{d t}=-\frac{N_{1}(t)}{\tau}-2 W_{E T U} N_{1}^{2}(t),
$$

which can be derived as,

$$
N_{1}(t)=\frac{N_{1}(0) \exp (-t / \tau)}{1+2 W_{E T U} N_{1}(0) \tau[1-\exp (-t / \tau)]},
$$


where $\tau$ is intrinsic lifetime of the excited state and $N_{1}(0)$ is density of excited ions upon cut-off of pump power. Equation 4.4 is generally known as Bernoulli's equation.

By simultaneously fitting the eight decay curves shown in Figure 4.5, a $W_{E T U}$ value of $1.3 \times 10^{-18} \mathrm{~cm}^{3} / \mathrm{s}$ is extracted. The $\tau$ obtained from the fit is $304 \mu \mathrm{s}$, which is higher than the value reported in previous subsection. This is possibly caused by suboptimal pinhole alignment and sample positioning during the experiment and it is deemed to cause little influence on the investigation of $W_{E T U}$. The presence of ETU process in $\mathrm{Yb}^{3+}$-activated material is not typical as $\mathrm{Yb}^{3+}$ ion has only two energy levels within the $4 f$-shell. Power dependent non-exponential decay behavior similar to the observation in current work had been reported in YbAG crystals [140] and it was attributed to cooperative energy transfer from two $\mathrm{Yb}^{3+}$ ions to an $\mathrm{Yb}^{2+}$ ion. Nevertheless, the presence of $\mathrm{Yb}^{2+}$ has not been reported in $\mathrm{KRE}\left(\mathrm{WO}_{4}\right)_{2}: \mathrm{Yb}^{3+}$ till date, although it is known to exist in certain as-grown crystals such as $\mathrm{YAG}: \mathrm{Yb}^{3+}[128]$ and LuAG: $\mathrm{Yb}^{3+}$ [141]. The observed ETU could be caused by cooperative upconversion luminescence, where the combined energy of the two excited ions leads to emission of visible photon, and possibly also cooperative or cumulative energy transfer to the impurities [142].
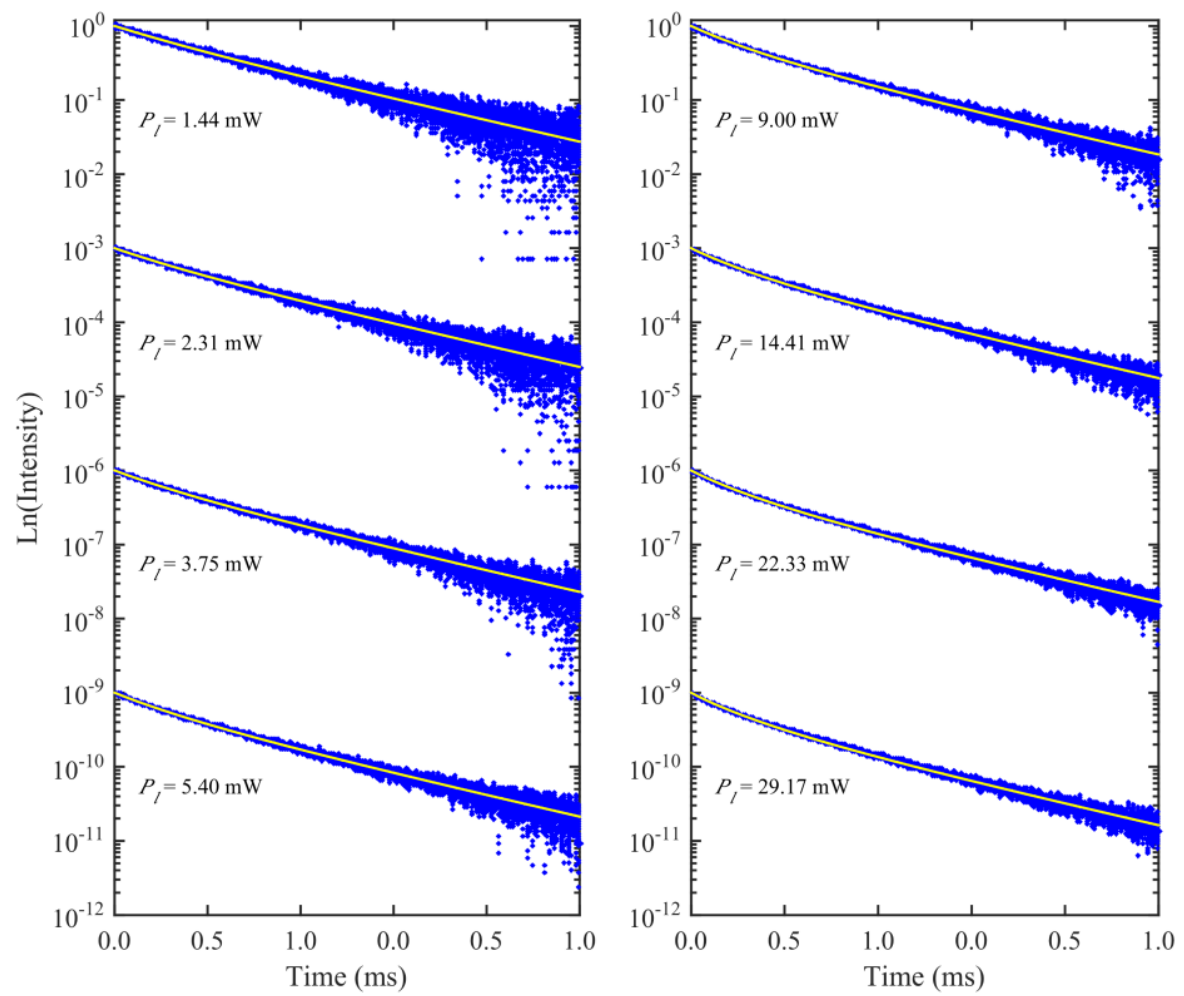

Figure 4.5 Luminescence decay curves measured with different launched pump power $P_{l}$ values (blue). The yellow lines represent the fitted curves using Bernoulli's equation (Eq. 4.4). 


\subsection{Absorption and emission cross-sections}

Thus far, experiment study on the transition cross-sections in $\mathrm{KRE}\left(\mathrm{WO}_{4}\right)_{2}: \mathrm{Yb}^{3+}$ epitaxial layer has been limited to layer with $\mathrm{Yb}^{3+} \leq 19$ at.\% [124]. In this section, absorption measurements are performed on the epitaxial layers with 57 at.\% and 76 at. $\% \mathrm{Yb}^{3+}$ to determine their transition cross-sections experimentally. The impact of stray light and the polarization disorientation on the measured absorption will be discussed. Besides, the transition cross-sections determined in the epitaxial layers will be compared to the results in the literature.

\subsubsection{Absorption measurement}

Absorption measurements are performed on two samples with thick epitaxial layers and high $\mathrm{Yb}^{3+}$ concentration prepared for amplifier experiments, namely the $\sim 32 \mu \mathrm{m}$ thick $\mathrm{KGd}_{0.43} \mathrm{Yb}_{0.57}\left(\mathrm{WO}_{4}\right)_{2}$ layer grown on undoped $\mathrm{KY}\left(\mathrm{WO}_{4}\right)_{2}$ substrate and the $\sim 124 \mu \mathrm{m}$ thick $\mathrm{KLu}_{0.24} \mathrm{Yb}_{0.76}\left(\mathrm{WO}_{4}\right)_{2}$ layer grown on undoped $\mathrm{KLu}\left(\mathrm{WO}_{4}\right)_{2}$ substrate. The rear surface of each sample was lapped and polished to remove the excess growth layer, whereas their respective front surface was lapped and polished parallel to the substrate. As these samples are highly absorbing, strong attenuation of the probe beam at the peak absorption wavelength is inevitable, resulting in an accordingly lowered signal-to-noise ratio. Consequently, stray light in the measurement system may result in a deceptively reduced absorption value and it should be considered in the experiment.

A dual-beam spectrophotometer (Shimadzu UV1800) with a spectral bandwidth of $1 \mathrm{~nm}$ is used together with a near-infrared (NIR) polarizer with $>400: 1$ extinction ratio (Thorlabs LPNIRE100-B) to determine the absorbance of both samples, as shown in Figure 4.6(a). Wavelength scans from $900 \mathrm{~nm}$ to $1050 \mathrm{~nm}$ with data acquisition in $0.1 \mathrm{~nm}$ steps are performed to determine the absorption due to $\mathrm{Yb}^{3+}$ ions. As the epitaxial layers were grown along the $N_{p}$ direction, absorption spectra with $E \| N_{m}$ and $E \| N_{g}$ polarization can be measured. The study of absorption is limited to $E \| N_{m}$ polarization, because this polarization exhibits the highest transition cross-sections and, therefore, is more commonly used for amplifiers and lasers than the $E \| N_{g}$ polarization. A series of measurements is performed while the polarizer is rotated by $\sim 180^{\circ}$ in steps to determine the sample orientation for absorption polarized to $E \| N_{m}$. The absorption spectrum for each angle step is corrected for the spectral response of the polarizer at the same angle as well as the Fresnel reflections of the sample. 

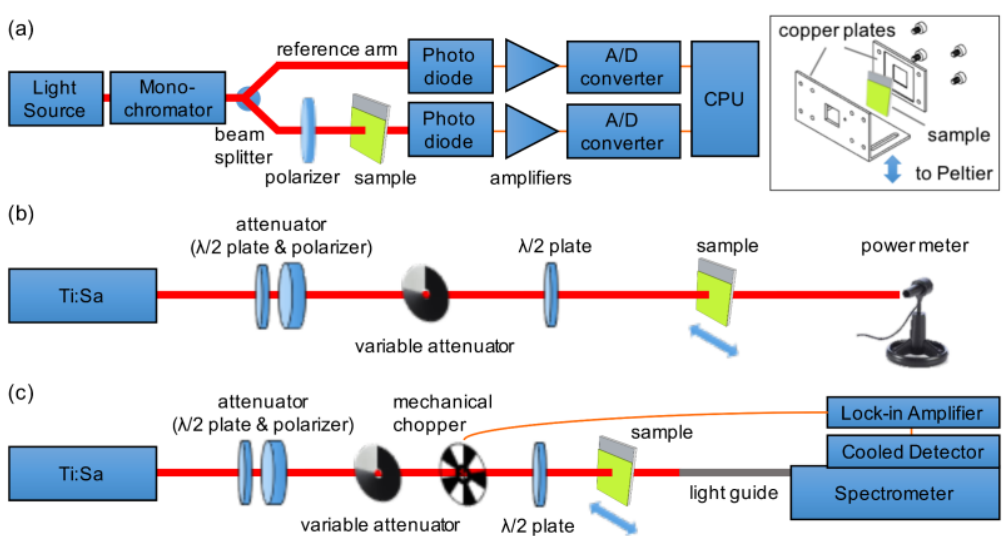

Figure 4.6 Schematic diagrams of (a) the commercial dual-beam spectrophotometer with a polarizer, (b) the free-space measurement setup with optical detection by a power meter, and (c) the free-space measurement setup with optical detection by a cooled detector attached to a spectrometer. The sample holder used for the temperature-dependent study is shown at the right of (a).

Figure 4.6(b) depicts the free-space setup with a Ti:Sapphire laser (SpectraPhysics 3900S, linewidth $<40 \mathrm{GHz}$ ) used for the measurement of the $\mathrm{KLu}_{0.24} \mathrm{Yb}_{0.76}\left(\mathrm{WO}_{4}\right)_{2}$ sample with an expected high total absorption of $\sim 35 \mathrm{~dB}$ at $981 \mathrm{~nm}$ wavelength. The intensity of the beam is attenuated to $<0.5 \mathrm{~W} / \mathrm{cm}^{2}$ over the entire measured wavelength range to ensure that it is much smaller than the saturation intensity of $5.4 \mathrm{~kW} / \mathrm{cm}^{2}$ [106] at $981 \mathrm{~nm}$. A broadband half-wave plate is used to rotate the polarization of the light source. In order to identify the $E \| N_{m}$ axis the transmission at $981 \mathrm{~nm}$ wavelength is measured and the half-wave plate is rotated until a minimal power is transmitted through the sample. A silicon detector (Coherent OP-2 VIS) with a specified detection range of $\sim 50 \mathrm{~dB}$ at $1000 \mathrm{~nm}$ wavelength is used to measure the transmitted optical power as the sample is positioned within/out of the beam path. The probe wavelength is tuned manually with tuning steps of $\leq 5 \mathrm{~nm}$. The tuning step is gradually reduced to $1 \mathrm{~nm}$ and then $0.25 \mathrm{~nm}$ near the absorption peaks at $932 \mathrm{~nm}$ and $981 \mathrm{~nm}$, respectively, to minimize the data acquisition time while ensuring that the absorption peaks are well resolved. For each wavelength step, three data points are recorded and an averaged absorption value is deduced.

Figure 4.6(c) shows the variation of the free-space setup to further minimize the influence of stray light, which is the undesirable light at other wavelengths. After passing through the sample, the probe beam is passed through a spectrometer (Jobin Yvon iHR550). The detection wavelength is tuned to the probe-beam wavelength, hence any residual emission from the laser source at other wavelengths is effectively discriminated from detection. A cooled detector and lock-in amplification are used to increase the signal-to-noise ratio. 


\subsubsection{Factors affecting the measurement results}

Careful measurement of the absorption in the active media is essential as this determines the accuracy of the peak transition cross-section values [143]. In view of the discrepancy on the peak absorption cross-section values in bulk $\mathrm{Yb}^{3+}$-doped potassium double tungstates, which varies from $7.1 \times 10^{-20} \mathrm{~cm}^{2}$ to $13.3 \times 10^{-20} \mathrm{~cm}^{2}[71,127]$, extra care is taken to avoid possible errors in the absorption measurement [143] by (i) employing a polarizer with high extinction ratio over the wavelength range under investigation, (ii) using a measurement system with spectral bandwidth resolution of $1 \mathrm{~nm}$ or better, and (iii) avoiding depletion of active ions in the ground state via proper control of the intensity of the probe beam. In addition, the impact of the polarization disorientation and the stray light to the measured absorption spectrum is also studied.

The absorption spectra of the $\mathrm{KGd}_{0.43} \mathrm{Yb}_{0.57}\left(\mathrm{WO}_{4}\right)_{2}$ sample measured at different polarization angle using the dual-beam spectrophotometer are shown in Figure 4.7. The inset shows the change of the magnitude of the peak absorption to the angle of polarizer, where the $E \| N_{m}$ is identified by the maximum absorbance at the central absorption line near $981 \mathrm{~nm}$. This peak absorption value follows a sinusoidal-like trend as the angle is changed. A comparison of the spectrum at $E \| N_{m}$ (blue line in Figure 4.7) to the spectrum obtained when the polarizer is oriented $16^{\circ}$ towards $E \| N_{g}$ (orange line in Figure 4.7) shows that the overall spectrum remains very similar despite the rather large angle difference, with the exception of the main absorption peak near $981 \mathrm{~nm}$ which is reduced by $\sim 10 \%$. For a smaller angle disorientation of $8^{\circ}$, the magnitude of the absorbance of this peak is reduced by $\sim 4 \%$ as compared to its magnitude at $E \| N_{m}$. These results show a less prominent impact of angle disorientation as compare to a previous report which suggested $\sim 20 \%$ error on the measured absorption at merely $2^{\circ}$ of disorientation [144]. Nevertheless, the results show that it is difficult to identify a small polarization disorientation unless a systematic study is performed.

Figure 4.8 shows the absorption spectra of the $\mathrm{KLu}_{0.24} \mathrm{Yb}_{0.76}\left(\mathrm{WO}_{4}\right)_{2}$ sample measured with the different setups at $E \| N_{m}$. In the case of the spectrophotometer, the ratio between the peak absorption near $981 \mathrm{~nm}$ to the peak absorption near $932 \mathrm{~nm}$ is only $\sim 1.7$, in comparison to the ratio of $\sim 4.7$ in $\mathrm{KGd}_{0.43} \mathrm{Yb}_{0.57}\left(\mathrm{WO}_{4}\right)_{2}$ observed in Figure 4.7. The maximum measured total absorbance of the sample and polarizer is merely 2.6 optical density (O.D.), though the measurement instrument has a specification of 4 O.D. The reason for such effect may be due to the stray light from the dispersive grating element which scatters a small amount of light at other wavelengths [145], which is a known issue for measurements on samples with high absorbance. As the spectrophotometer is tuned to $981 \mathrm{~nm}$, the signal is heavily attenuated, whereas the stray light at other wavelengths experiences little or no absorption. Hence, the total intensity recorded by the detector at the peak is higher than the true transmitted intensity at $981 \mathrm{~nm}$ and the system produces a lower absorption reading. 


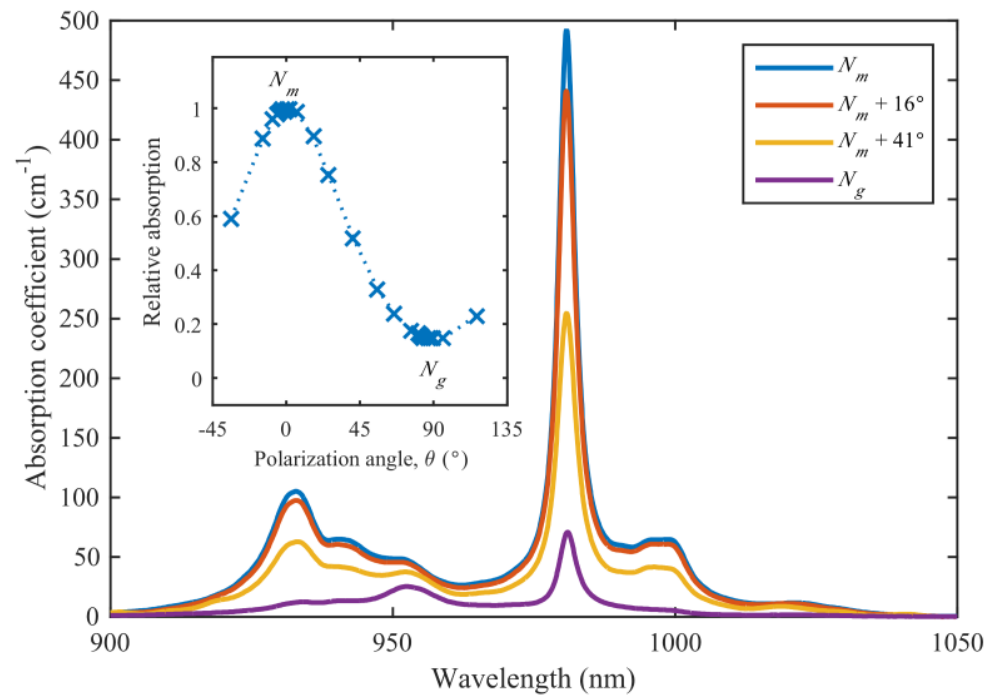

Figure 4.7 Absorption spectra of $\mathrm{KGd}_{0.43} \mathrm{Yb}_{0.57}\left(\mathrm{WO}_{4}\right)_{2}$ measured with the spectrophotometer at various angles of polarization of the incident beam. The inset shows the change of peak absorption, relative to the peak absorption at $E \| N_{m}$ axis, to the polarization angle.

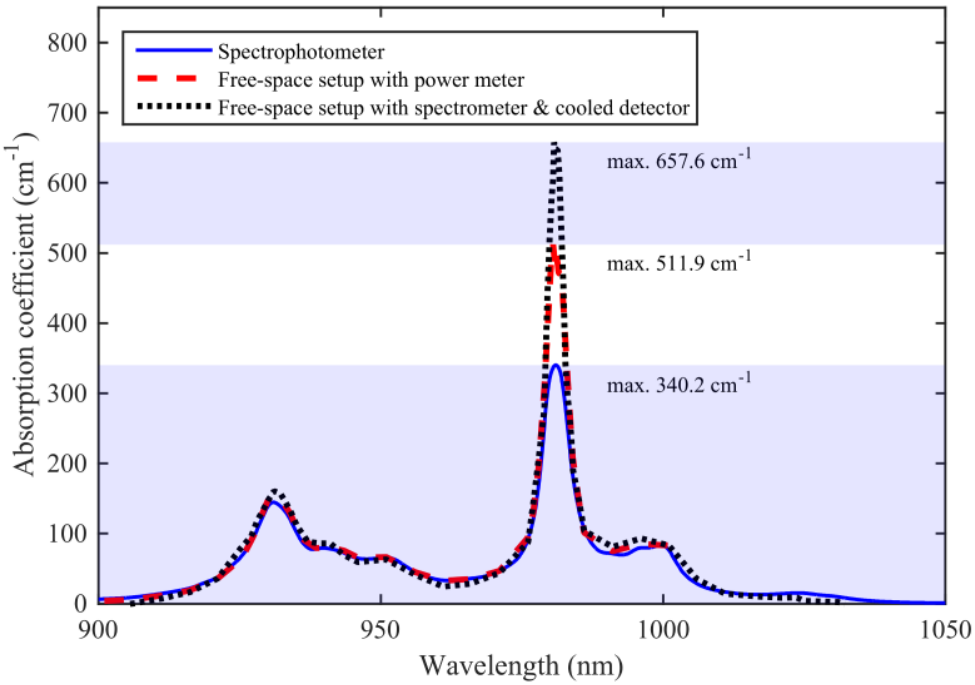

Figure 4.8 Absorption spectra polarized to $E \| N_{m}$ for the $\mathrm{KLu}_{0.24} \mathrm{Yb}_{0.76}\left(\mathrm{WO}_{4}\right)_{2}$ sample with high total absorbance measured using the spectrophotometer (blue solid curve), free-space setup with power meter (red dashed curve), and free-space setup with cooled detector attached to a spectrometer (black dotted curve). 
The stray-light issue can be circumvented by use of higher signal power or a light source with better signal-to-noise ratio. To verify if the stray light played a role in the absorption result measured with the spectrophotometer, the free-space setup displayed in Figure 4.6(b) is used to repeat the measurement in $\mathrm{KLu}_{0.24} \mathrm{Yb}_{0.76}\left(\mathrm{WO}_{4}\right)_{2}$. The result, displayed in Figure 4.8 as the dashed curve, shows a better-resolved absorption peak with a maximum absorption coefficient of $512 \mathrm{~cm}^{-1}$. Nevertheless, when the probe beam is tuned to $981 \mathrm{~nm}$, the wavelength spectrum collected after the sample still reveals some detectable residual NIR luminescence $(\sim 700-850 \mathrm{~nm})$ from the Ti:Sapphire laser crystal. In order to further suppress the stray light, the probe beam is sent to the spectrometer equipped with a cooled detector, as shown in Figure 4.6(c). Using this setup, the peak-absorption coefficient is well resolved and approaches $658 \mathrm{~cm}^{-1}$ (see Figure 4.8, dotted curve), which is nearly two times the initial value obtained with the spectrophotometer. Applying the calculation method described in [145], the respective amount of stray light in the spectrophotometer, the free-space setup with power meter, and the free-space setup with spectrometer and cooled detector are approximated as $\sim 0.25 \%, \sim 0.15 \%$, and $<0.002 \%$ of the probe-beam intensity at $981 \mathrm{~nm}$. It is evident that a small amount of stray light may lead to a deceptive peak absorption value in sample with high total absorbance.

\subsubsection{Evaluation of transition cross-sections}

The effective absorption cross-section $\sigma_{a b s}$ is calculated from the measured absorption coefficient $\alpha$ and the known $\mathrm{Yb}^{3+}$ concentration $N_{Y b}$, Subsequently, the effective emission cross-section $\sigma_{e m}$ is calculated from $\sigma_{a b s}$ using reciprocity method as explained in Section 2.3.4. The level energies of the $\mathrm{KY}\left(\mathrm{WO}_{4}\right)_{2}: \mathrm{Yb}^{3+}$ [71] are used for the calculation of $\sigma_{e m}$. As mentioned in Section 3.2.2, the difference between the level energies among the potassium double tungstates are small and the calculated $\sigma_{e m}$ would be very similar if any other set of level energies are used.

The respective room temperature $\sigma_{a b s}$ and $\sigma_{e m}$ for both samples at $E \| N_{m}$ are shown in Figure 4.9 and Figure 4.10. For the calculation of $\sigma_{a b s}$ in $\mathrm{KLu}_{0.24} \mathrm{Yb}_{0.76}\left(\mathrm{WO}_{4}\right)_{2}$, the $N_{Y b}$ value of $5.0 \times 10^{21} \mathrm{~cm}^{-3}$ is used. The absorption data is taken from Figure 4.8, using the spectrophotometer measurement result in the low-absorption range and the measurement results from the free-space setup with spectrometer and cooled detector at the peak-absorption region $(970-985 \mathrm{~nm})$ to retain data with good signal-to-noise ratio over the entire spectrum. The $\sigma_{a b s}$ in $\mathrm{KGd}_{0.43} \mathrm{Yb}_{0.57}\left(\mathrm{WO}_{4}\right)_{2}$ is calculated from $N_{Y b}$ of $3.8 \times 10^{21} \mathrm{~cm}^{-3}$ and the absorption spectrum measured with the spectrophotometer setup. As the thickness and the $\mathrm{Yb}^{3+}$ concentration of the $\mathrm{KGd}_{0.43} \mathrm{Yb}_{0.57}\left(\mathrm{WO}_{4}\right)_{2}$ sample are only $\sim 1 / 4$ and $\sim 3 / 4$ of the $\mathrm{KLu}_{0.24} \mathrm{Yb}_{0.76}\left(\mathrm{WO}_{4}\right)_{2}$ sample, respectively, the corresponding total absorption is much lower. Therefore, the stray light is not 
significant at the absorption peak near $981 \mathrm{~nm}$ and it is sufficient to use only the spectrophotometer setup for the $\mathrm{KGd}_{0.43} \mathrm{Yb}_{0.57}\left(\mathrm{WO}_{4}\right)_{2}$ sample.

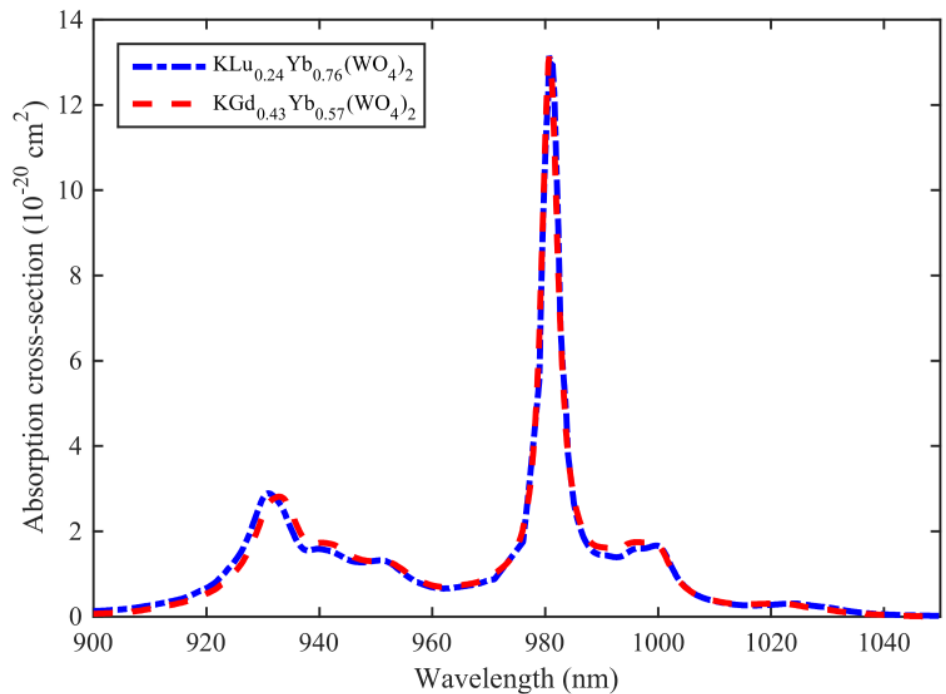

Figure 4.9 Room temperature effective absorption cross-section $\sigma_{a b s}$ in the samples of $\mathrm{KLu}_{0.24} \mathrm{Yb}_{0.76}\left(\mathrm{WO}_{4}\right)_{2}$ and $\mathrm{KGd}_{0.43} \mathrm{Yb}_{0.57}\left(\mathrm{WO}_{4}\right)_{2}$.

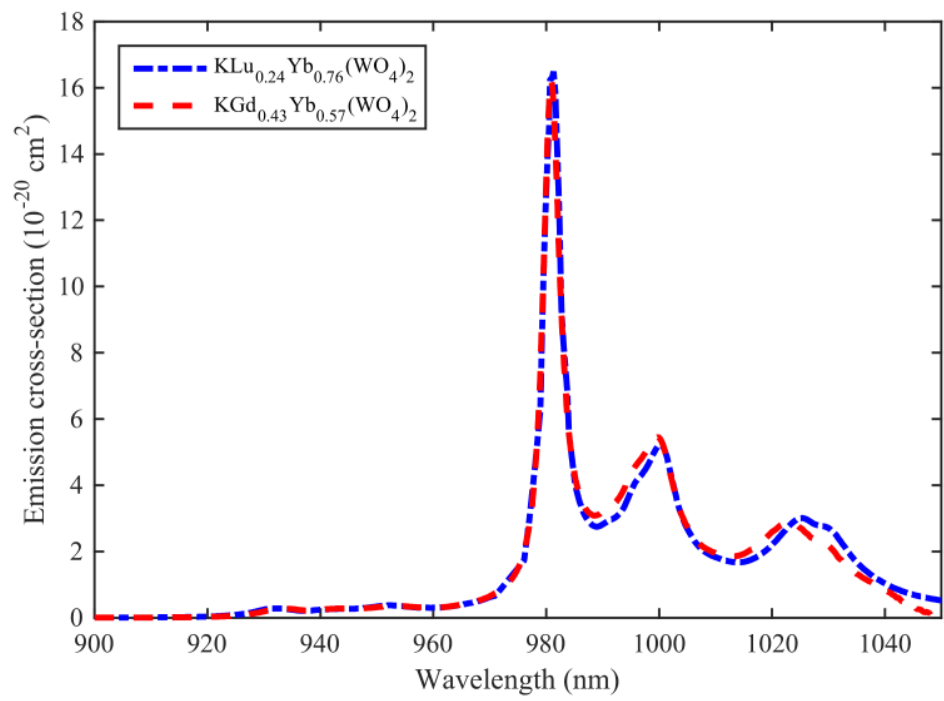

Figure 4.10 Room temperature effective emission cross-section $\sigma_{e m}$ in the samples of $\mathrm{KLu}_{0.24} \mathrm{Yb}_{0.76}\left(\mathrm{WO}_{4}\right)_{2}$ and $\mathrm{KGd}_{0.43} \mathrm{Yb}_{0.57}\left(\mathrm{WO}_{4}\right)_{2}$. 
The calculated peak $\sigma_{a b s}$ and $\sigma_{e m}$ are $1.32 \times 10^{-19} \mathrm{~cm}^{2}$ and $1.65 \times 10^{-19} \mathrm{~cm}^{2}$, respectively, for $\mathrm{KLu}_{0.24} \mathrm{Yb}_{0.76}\left(\mathrm{WO}_{4}\right)_{2}$, while the respective peak $\sigma_{a b s}$ and $\sigma_{e m}$ for $\mathrm{KGd}_{0.43} \mathrm{Yb}_{0.57}\left(\mathrm{WO}_{4}\right)_{2}$ are $1.31 \times 10^{-19} \mathrm{~cm}^{2}$ and $1.62 \times 10^{-19} \mathrm{~cm}^{2}$. The full width at half maximum (FWHM) of the central absorption line near $981 \mathrm{~nm}$ wavelength is $3.7 \mathrm{~nm}$ for $\mathrm{KLu}_{0.24} \mathrm{Yb}_{0.76}\left(\mathrm{WO}_{4}\right)_{2}$ and $3.8 \mathrm{~nm}$ for $\mathrm{KGd}_{0.43} \mathrm{Yb}_{0.57}\left(\mathrm{WO}_{4}\right)_{2}$. Despite the large variation of the $\mathrm{Yb}^{3+}$ at.\% and being grown on a dissimilar type of substrate, the transition crosssections in these samples are very close to each other.

\subsubsection{Discussions}

Since the radiation trapping is pronounced in $\mathrm{KRE}\left(\mathrm{WO}_{4}\right)_{2}: \mathrm{Yb}^{3+}$, most the reported $\sigma_{e m}$ spectra were calculated from the reciprocity method [86] instead of the FüchtbauerLadenburg equation $[88,90,91]$. Therefore, the benchmarking of the transition crosssections obtained in the previous sub-section can be done by comparing only the $\sigma_{a b s}$ to those reported in the literature, since the $\sigma_{e m}$ can be readily calculated from the $\sigma_{a b s}$.

The absorption spectra of $\mathrm{KLu}_{0.24} \mathrm{Yb}_{0.76}\left(\mathrm{WO}_{4}\right)_{2}$ and $\mathrm{KGd}_{0.43} \mathrm{Yb}_{0.57}\left(\mathrm{WO}_{4}\right)_{2}$ shown in Figure 4.9 are found to be very similar to those of $\mathrm{KGd}\left(\mathrm{WO}_{4}\right)_{2}: \mathrm{Yb}^{3+}[73,114]$, $\mathrm{KLu}\left(\mathrm{WO}_{4}\right)_{2}: \mathrm{Yb}^{3+}[107]$, and $\mathrm{KYb}\left(\mathrm{WO}_{4}\right)_{2}$ [106]. They are in excellent agreement with that of $\mathrm{KY}\left(\mathrm{WO}_{4}\right)_{2}: \mathrm{Yb}^{3+*}[71]$, especially at the peak absorption value near $981 \mathrm{~nm}$. Table 4.3 compares the peak $\sigma_{a b s}$ derived from the high $\mathrm{Yb}^{3+}$ concentration epitaxial layers to those of bulk $\mathrm{KRE}\left(\mathrm{WO}_{4}\right)_{2}: \mathrm{Yb}^{3+}$ crystals. The peak $\sigma_{a b s}$ values determined in this work favorably match the value of $1.33 \times 10^{-19} \mathrm{~cm}^{2}$ in $\mathrm{KY}\left(\mathrm{WO}_{4}\right)_{2}: \mathrm{Yb}^{3+}$ [71], though lower cross-section values ranging from $0.71 \times 10^{-19} \mathrm{~cm}^{2}$ to $1.17 \times 10^{-19} \mathrm{~cm}^{2}$ had also been reported for similar material $[105,127]$. The reported peak $\sigma_{a b s}$ values in other $\mathrm{KRE}\left(\mathrm{WO}_{4}\right)_{2}: \mathrm{Yb}^{3+}$, i.e. $\mathrm{KGd}\left(\mathrm{WO}_{4}\right)_{2}: \mathrm{Yb}^{3+}[71], \mathrm{KLu}\left(\mathrm{WO}_{4}\right)_{2}: \mathrm{Yb}^{3+}[74,107]$, and $\mathrm{KYb}\left(\mathrm{WO}_{4}\right)_{2}[104,106]$, are $\sim 1.2 \times 10^{-19} \mathrm{~cm}^{2}$. The slight differences among the reported peak $\sigma_{a b s}$ values may be attributed to the spectral resolution of the different measurement systems and/or the measurement uncertainty of the doping concentration values. In overall, the spectroscopic properties of the highly $\mathrm{Yb}^{3+}$-doped thin films are comparable to those in bulk $\mathrm{KRE}\left(\mathrm{WO}_{4}\right)_{2}: \mathrm{Yb}^{3+}$ and $\mathrm{KYb}\left(\mathrm{WO}_{4}\right)_{2}$ crystals.

\footnotetext{
${ }^{*}$ The calculated $\sigma_{e m}$, on the other hand, are different from the $\sigma_{e m}$ in $\mathrm{KY}\left(\mathrm{WO}_{4}\right)_{2}:\left(\sim 5\right.$ at.\%) $\% \mathrm{Yb}^{3+}$ presented by Kuleshov et al. [71]. Aravazhi et al. [65] showed that the $\sigma_{e m}$ given in [71] was possibly miscalculated.
} 
Table 4.3 Comparison of peak effective absorption cross-section $\sigma_{\text {peak }}$ as well as the corresponding wavelength $\lambda_{\text {peak }}$ and FWHM of the absorption peak for $\mathrm{Yb}^{3+}$-doped potassium rare-earth double tungstate crystals and epitaxial layer.

\begin{tabular}{|c|c|c|c|c|c|}
\hline Material & $\begin{array}{c}\text { Bulk/ } \\
\text { Epitaxial } \\
\text { layer }\end{array}$ & $\begin{array}{c}\mathbf{Y b}^{3+} \\
\text { concentration } \\
\left(10^{20} \mathrm{~cm}^{-3}\right)\end{array}$ & $\begin{array}{c}\sigma_{\text {peak }} \\
\left(10^{-19} \mathrm{~cm}^{2}\right)\end{array}$ & $\begin{array}{l}\lambda_{p e a k} \\
(\mathbf{n m})\end{array}$ & $\begin{array}{l}\text { FWHM * } \\
\quad(\mathbf{n m})\end{array}$ \\
\hline $\mathrm{KGd}\left(\mathrm{WO}_{4}\right)_{2}: \mathrm{Yb}^{3+}, \mathrm{Er}^{3+}[73]$ & Bulk & $0.173^{\mathrm{a}}$ & 1.16 & 980.8 & N/A \\
\hline $\mathrm{KGd}\left(\mathrm{WO}_{4}\right)_{2}: \mathrm{Yb}^{3+}[114]$ & Bulk & 2 & $\sim 1.14^{\mathrm{b}}$ & $\sim 981.2$ & N/A \\
\hline $\mathrm{KGd}\left(\mathrm{WO}_{4}\right)_{2}: \mathrm{Yb}^{3+}[71]$ & Bulk & 2.2 & 1.20 & 981 & 3.7 \\
\hline $\mathrm{KGd}\left(\mathrm{WO}_{4}\right)_{2}: \mathrm{Yb}^{3+}[146]$ & Bulk & $\sim 3.2$ & $0.92^{b}$ & $\sim 981$ & N/A \\
\hline $\mathrm{KLu}\left(\mathrm{WO}_{4}\right)_{2}: \mathrm{Yb}^{3+}[74,107]$ & Bulk & 0.45 & 1.18 & 981.1 & 3.6 \\
\hline $\mathrm{KY}\left(\mathrm{WO}_{4}\right)_{2}: \mathrm{Yb}^{3+}, \mathrm{Er}^{3+}[105]$ & Bulk & $0.24^{\mathrm{c}}$ & 1.17 & 980.6 & N/A \\
\hline $\mathrm{KY}\left(\mathrm{WO}_{4}\right)_{2}: \mathrm{Yb}^{3+}[71]$ & Bulk & 3 & 1.33 & 981.2 & 3.5 \\
\hline $\mathrm{KY}\left(\mathrm{WO}_{4}\right)_{2}: \mathrm{Yb}^{3+}[127]$ & Bulk & $\sim 3.2$ & 0.71 & 981 & N/A \\
\hline $\mathrm{KYb}\left(\mathrm{WO}_{4}\right)_{2}[104,106]$ & Bulk & 64 & 1.17 & 981 & 4.0 \\
\hline $\mathrm{KY}_{0.81} \mathrm{Yb}_{0.19}\left(\mathrm{WO}_{4}\right)_{2} / \mathrm{KY}\left(\mathrm{WO}_{4}\right)_{2}[124]$ & Epitaxial & $19.92^{\mathrm{d}}$ & 1.25 & 981.8 & N/A \\
\hline $\begin{array}{l}\mathrm{KLu}_{0.24} \mathrm{Yb}_{0.76}\left(\mathrm{WO}_{4}\right)_{2} / \mathrm{KLu}\left(\mathrm{WO}_{4}\right)_{2} \\
\text { (this work) }\end{array}$ & Epitaxial & 50 & 1.32 & 980.7 & 3.7 \\
\hline $\begin{array}{l}\mathrm{KGd}_{0.43} \mathrm{Yb}_{0.57}\left(\mathrm{WO}_{4}\right)_{2} / \mathrm{KY}\left(\mathrm{WO}_{4}\right)_{2} \\
\text { (this work) }\end{array}$ & Epitaxial & 38 & 1.31 & 980.8 & 3.8 \\
\hline
\end{tabular}

* Since extraction of FHWM from the published data is prone to digitization error, only the FHWM specified in the original publications are listed in this table.

(a) Co-doped with $0.144 \times 10^{20} \mathrm{~cm}^{-3} \mathrm{Er}^{3+}$.

(b) Value calculated from the absorption data given in the cited work.

(c) Co-doped with $0.315 \times 10^{20} \mathrm{~cm}^{-3} \mathrm{Er}^{3+}$.

(d) The same research group had reported $\mathrm{Yb}^{3+}$ of $11.96 \times 10^{20} \mathrm{~cm}^{-3}$ for epitaxial layer with similar material composition [60]. The $\mathrm{Yb}^{3+}$ value of $19.92 \times 10^{20} \mathrm{~cm}^{-3}$ could be overestimated as this corresponds to $\sim 30$ at. $\% \mathrm{Yb}^{3+}$. Moreover, despite having peak $\sigma_{a b s}$ value matches to other published work, the $\sigma_{a b s}$ curve in [124] shows a clearly under-resolved peak absorption $\sim 981 \mathrm{~nm}$.

\subsection{Temperature dependence of spectroscopic parameters}

Spectroscopic measurements are repeated on $\mathrm{KGd}_{0.43} \mathrm{Yb}_{0.57}\left(\mathrm{WO}_{4}\right)_{2}$ with various temperature settings to emulate the behavior of these properties when heat is generated by the amplifier. A homemade copper sample holder in contact with a Peltier element as shown in Figure 4.6(a) is used during these measurements. The temperature of the sample holder is regulated using a thermoelectric temperature controller (Melcor MTTC1410). The maximum temperature on the sample holder is $\sim 170{ }^{\circ} \mathrm{C}$, which is limited by the Peltier element used in the experiment. 


\subsubsection{Temperature dependence of luminescent lifetime}

The luminescent decay curves are measured with the same confocal method described in Section 4.2.2 at low launched pump power at various temperature settings. The lifetime values extracted from the decay curves are plotted in Figure 4.11. The lifetime values derived from two measurement series are $230 \pm 7 \mu \mathrm{s}$ and $220 \pm 4 \mu \mathrm{s}$, respectively, and the maximum deviation from the mean values is $<5 \%$. The data shows no significant dependence of the measured lifetime on the temperature within the range of temperature investigated. In other words, the thermal quenching of lifetime is not apparent.

The result is within expectation because the temperature dependence of lifetime is primarily contributed by multiphonon relaxation [84]. Since the energy gap between the ground state and the excited state is $\sim 10000 \mathrm{~cm}^{-1}$ (see Figure 3.2) while the highest phonon energy in $\mathrm{KRE}\left(\mathrm{WO}_{4}\right)_{2}: \mathrm{Yb}^{3+}$ is $908 \mathrm{~cm}^{-1}$ [107], more than 10 phonons are needed to bridge the gap. Therefore, the probability of multiphonon relaxation is inherently small. The finding that the lifetime has no significant dependence on the temperature is consistent with the reported lifetime determined from the luminescence decay of the ${ }^{4} \mathrm{~F}_{3 / 2}$ state in $\mathrm{KG}\left(\mathrm{WO}_{4}\right)_{2}: \mathrm{Nd}^{3+}$ which was nearly independent to the temperature within $77-450 \mathrm{~K}$ [147].

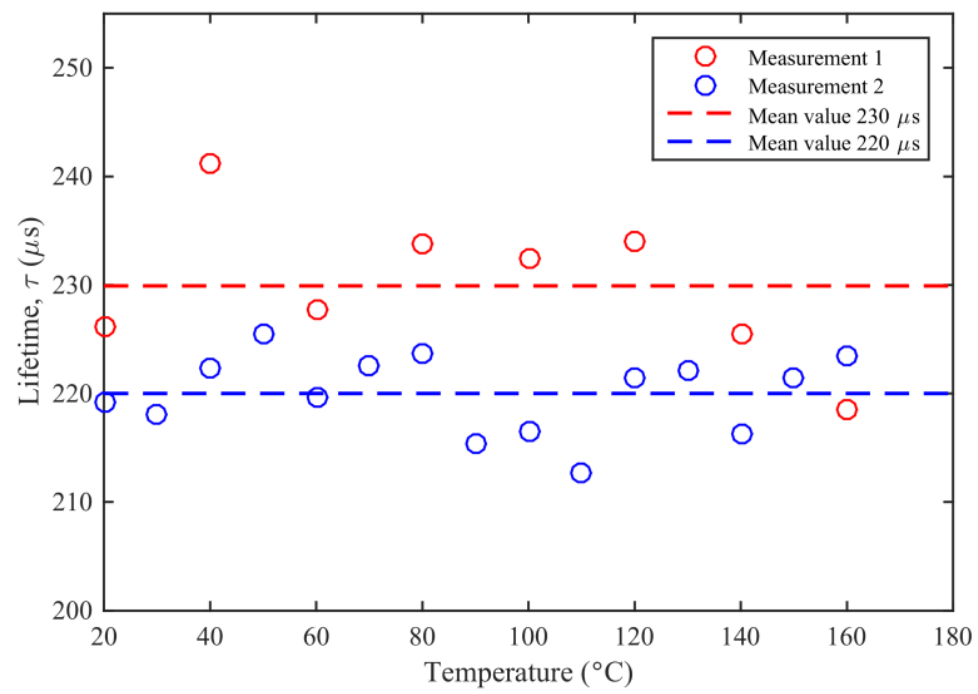

Figure 4.11 Temperature dependence of luminescence lifetime in $\operatorname{KGd}_{0.43} \mathbf{Y b}_{0.57}\left(\mathrm{WO}_{4}\right)_{2}$. 


\subsubsection{Temperature dependence of cross-sections}

The absorption in the sample is measured using the spectrophotometer setup as explained in Section 4.3.1 to deduce the temperature dependence of the transition crosssections. The results for $\sigma_{a b s}$ is shown in Figure 4.12(a). As the temperature is increased, the central absorption line near $981 \mathrm{~nm}$ reduces rapidly. The wavelength corresponding to the peak is slightly blue-shifted from $980.8 \mathrm{~nm}$ to $980.5 \mathrm{~nm}$. A similar, but less drastic, reduction of absorption is also noted at the peak near $932 \mathrm{~nm}$. However, the corresponding peak wavelength for this transition is shifted more from $932.9 \mathrm{~nm}$ at $20{ }^{\circ} \mathrm{C}$ to $931.7 \mathrm{~nm}$ at $170{ }^{\circ} \mathrm{C}$. The corresponding $\sigma_{e m}$ spectra calculated with the reciprocity method are displayed in Figure 4.12(b). As expected, the magnitude of the emission line near $981 \mathrm{~nm}$ is also strongly dependent on the temperature.

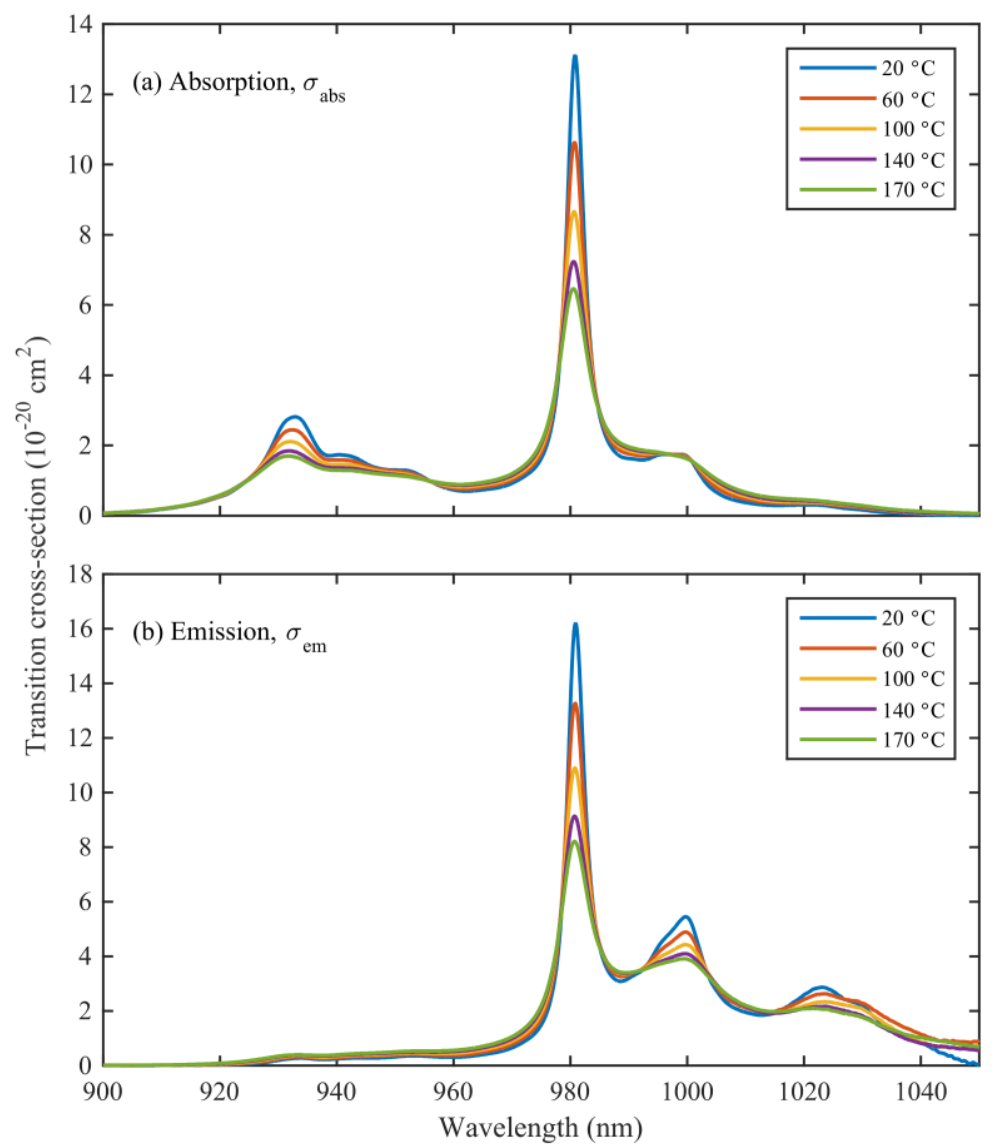

Figure 4.12 Temperature dependence of (a) absorption, and (b) emission cross-sections in $\mathrm{KGd}_{0.43} \mathrm{Yb}_{0.57}\left(\mathrm{WO}_{4}\right)_{2}$. 


\subsubsection{Temperature dependence of major absorption lines}

The strong temperature dependence of the peak $\sigma_{a b s}$ at $932 \mathrm{~nm}$ and $981 \mathrm{~nm}$ shown in Figure 4.12(a) is further investigated by considering peak decomposition using multiple peaks fitting with the aid of a data analysis program (Origin 9.1). As the sample is crystalline in nature, Lorentzian function is used to fit the peaks. Representative fitted absorption curves measured at $20^{\circ} \mathrm{C}$ and $170{ }^{\circ} \mathrm{C}$ are depicted in Figure 4.13.

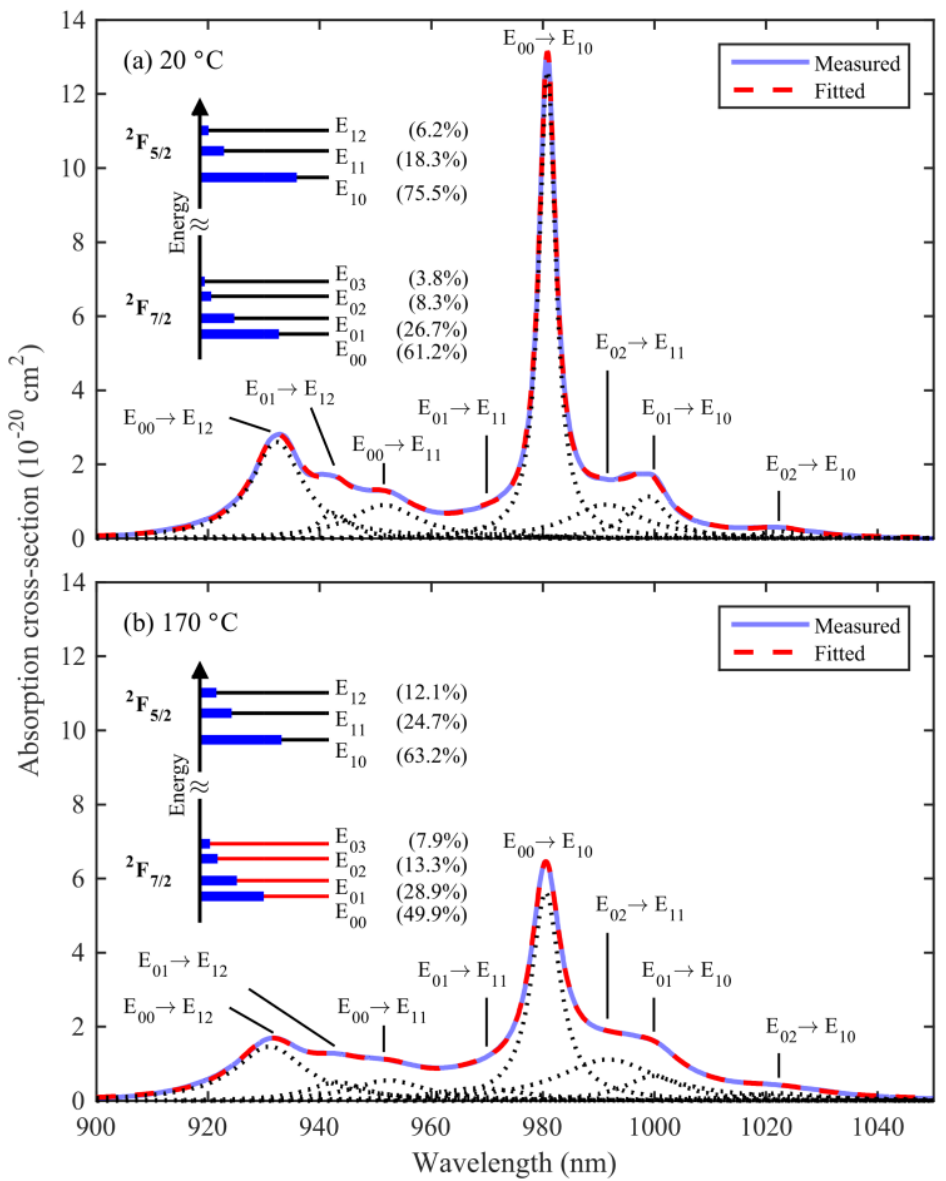

Figure 4.13 Multi-peak fitted absorption cross-section at (a) $20^{\circ} \mathrm{C}$ and (b) $170{ }^{\circ} \mathrm{C}$. The dotted lines show the decomposed peaks corresponding to different inter-Stark transitions. The eight transitions wavelengths used for the fitting are labeled and positioned near to the decomposed peaks. The insets show the level energies of $\mathrm{KY}\left(\mathrm{WO}_{4}\right)_{2}: \mathbf{Y b}^{3+}[71]$. The fractional populations calculated at the given temperature are represented by the blue thick horizontal lines and their values are shown on the right to their respective Stark level. 
The insets of Figure 4.13 show the level energies of $\mathrm{KY}\left(\mathrm{WO}_{4}\right)_{2}: \mathrm{Yb}^{3+}$ and the calculated fractional populations at each level at the given temperature. The 8 out of 12 total possible inter-Stark transitions used for the fitting are also labelled in the figure. In the event of overlapping transitions, we consider the transition that involves lower Stark levels to be more relevant, assuming that both transition strengths are similar. For instance, the $E_{02} \rightarrow E_{12}$ transition ( $\sim 970 \mathrm{~nm}$ ) overlaps with the more prominent $E_{01} \rightarrow E_{11}$ transition, therefore it is not treated as an independent peak. This also applies to the $E_{03}$ $\rightarrow E_{12}(\sim 982 \mathrm{~nm})$ and $E_{03} \rightarrow E_{11}(\sim 1005 \mathrm{~nm})$ transitions, which are overshadowed by the $E_{00} \rightarrow E_{10}$ and $E_{01} \rightarrow E_{10}$ transitions, respectively, due to much lower fractional population of the $E_{03}$ Stark level. The remaining $E_{03} \rightarrow E_{10}$ transition is not considered in the fitting, because the measured absorption is less apparent compared to other peaks. Besides, excluding this transition in the fitting has negligible impact on the following studies, because the corresponding transition wavelength of $\sim 1040 \mathrm{~nm}$ is far from the $932 \mathrm{~nm}$ and $981 \mathrm{~nm}$ wavelengths.

Based on Figure 4.13(b), it is observed that the peak absorption is well described by the Lorentzian shape even at $170{ }^{\circ} \mathrm{C}$, thereby confirming the dominance of homogeneous broadening over the measurement range. Besides, the absorption peaks at $932 \mathrm{~nm}$ and $981 \mathrm{~nm}$ dominate even at $170{ }^{\circ} \mathrm{C}$. For both cases, the magnitudes of their respective neighboring transitions are relatively weak. Hence, it is reasonable to assume that the influence of neighboring transitions is negligible and the temperature dependence of these major absorption peaks can be approximated from Equation 2.24 which is reproduced as follows,

$$
\sigma_{a b s}(T)=\sigma_{a b s}\left(T_{0}\right) \frac{f_{00}(T)}{f_{00}\left(T_{0}\right)} \frac{\Delta v\left(T_{0}\right)}{\Delta v(T)} .
$$

Figure 4.14(a) shows the value of $f_{00}$ at various temperatures, calculated based on the level energies of $\mathrm{KY}\left(\mathrm{WO}_{4}\right)_{2}: \mathrm{Yb}^{3+}$. The values deviate by less than $2.5 \%$ if any of the other sets of level energies of $\mathrm{KGd}\left(\mathrm{WO}_{4}\right)_{2}: \mathrm{Yb}^{3+}[73], \mathrm{KLu}\left(\mathrm{WO}_{4}\right)_{2}: \mathrm{Yb}^{3+}$ [74] or $\mathrm{KYb}\left(\mathrm{WO}_{4}\right)_{2}[104]$ is chosen. When the temperature increases from $20{ }^{\circ} \mathrm{C}$ to $170{ }^{\circ} \mathrm{C}$, the value of $f_{00}$ is reduced by $\sim 18 \%$. Figure 4.14 (b) displays the extracted FWHM of the decomposed absorption peaks near $932 \mathrm{~nm}$ and $981 \mathrm{~nm}$, indicating that their FWHM at the highest temperature is $\sim 1.37$ and $\sim 1.72$ times broader at $170{ }^{\circ} \mathrm{C}$ than at $20^{\circ} \mathrm{C}$, respectively. The corresponding ratio of $\Delta v\left(20^{\circ} \mathrm{C}\right) / \Delta v\left(170^{\circ} \mathrm{C}\right)$ is $\sim 0.73$ and $\sim 0.58$ for the respective transitions at $932 \mathrm{~nm}$ and $981 \mathrm{~nm}$. 

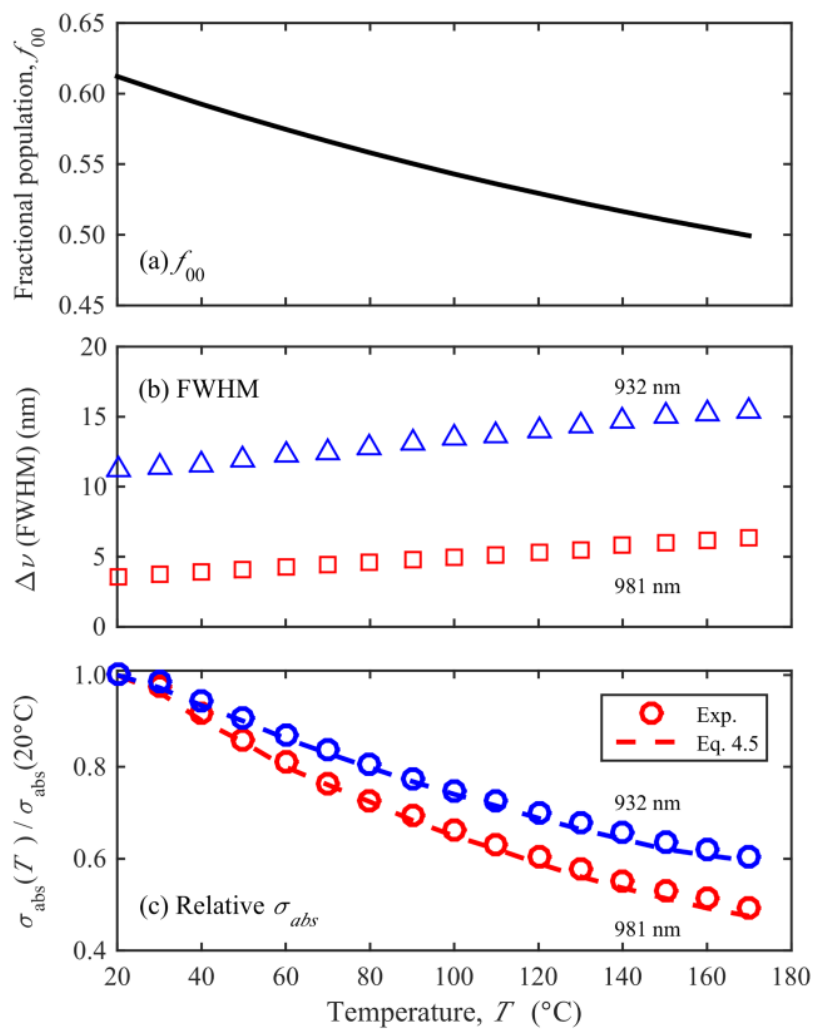

Figure 4.14 Spectroscopic data as a function of temperature: (a) calculated fractional population $f_{00}$, (b) extracted $\Delta v$ (FWHM) of the absorption peaks near $932 \mathrm{~nm}$ and $981 \mathrm{~nm}$, and (c) relative change of peak-absorption crosssection $\sigma_{a b s}$ of the transitions near $932 \mathrm{~nm}$ and $981 \mathrm{~nm}$ in $\mathrm{KGd}_{0.43} \mathrm{Yb}_{0.57}\left(\mathrm{WO}_{4}\right)_{2}$ and the calculated curves using Eq. 4.5.

The change of the peak-absorption cross-sections at elevated temperatures with respect to $20{ }^{\circ} \mathrm{C}, \sigma_{a b s}(T) / \sigma_{a b s}\left(20^{\circ} \mathrm{C}\right)$, is shown in Figure $4.14(\mathrm{c})$. A reduction by $\sim 40 \%$ and $\sim 52 \%$ for the transitions near $932 \mathrm{~nm}$ and $981 \mathrm{~nm}$, respectively, occurs. The result from the simple model of Eq. 4.5 is in good agreement with the measured reduction of peak magnitudes, confirming that the origin of the reduction of peak-absorption crosssection with temperature is a combination of the decrease of the population at the starting Stark level and the widening of the transition linewidth with temperature. In the high-temperature range, the simple model starts to deviate from the measurement points and the contributions from adjacent peaks need to be taken into account.

The simple model of Eq. 4.5 is further examined by considering the temperature dependent absorption cross-section data reported for $\mathrm{KG}\left(\mathrm{WO}_{4}\right)_{2}: \mathrm{Nd}^{3+}$ [147]. At $300 \mathrm{~K}$, the peak absorption cross-section at $810.5 \mathrm{~nm}$ wavelength (corresponds to the ${ }^{4} \mathrm{I}_{9 / 2} \rightarrow$ ${ }^{4} \mathrm{~F}_{5 / 2}$ transition) for polarization $E \| N_{m}$ was $29 \times 10^{-20} \mathrm{~cm}^{2}$ and the corresponding FWHM 
of the absorption peak was $1.7 \mathrm{~nm}$. The absorption cross-section decreased to $\sim 16.11 \times 10^{-20} \mathrm{~cm}^{2}$ at $450 \mathrm{~K}$ and the FWHM of the peak was broadened to $2.7 \mathrm{~nm}$. Using the level energies of $\mathrm{KG}\left(\mathrm{WO}_{4}\right)_{2}: \mathrm{Nd}^{3+}$ reported in [148], $f_{00}(300 \mathrm{~K})$ and $f_{00}(450 \mathrm{~K})$ are calculated as 0.3939 and 0.3260 , respectively. Therefore, the ratio between the peak absorption cross-sections is

$$
\frac{\sigma_{a b s}(450 K)}{\sigma_{a b s}(300 K)}=\frac{f_{00}(450 K)}{f_{00}(300 K)} \frac{\Delta v(300 K)}{\Delta v(450 K)}=\frac{0.3260}{0.3939} \cdot \frac{1.7}{2.7}=0.52,
$$

which is in good agreement to the ratio of $\sim 0.56$ obtained by directly dividing the absorption cross-section values.

\section{Origin of linewidth broadening}

Given the substantial influence of linewidth broadening on the temperature dependence of peak-absorption cross-sections, understanding the origin of linewidth broadening may help to generate a simple model that can describe the temperature dependence over a wide range of temperatures. The measured linewidth $\Delta v$ is caused by intra-manifold transitions due to electron-phonon coupling on the fs time scale [89] which establishes the Boltzmann distribution. Considering a single-phonon contribution to the homogeneous broadening, i.e., the transition is accompanied by the absorption/emission of one phonon, a simplified expression can be derived from the detailed calculation of the electron-phonon interaction for non-adiabatic systems [149, 150],

$$
\Delta v(T) \propto\left[\exp \left(h v / k_{B} T\right)-1\right]^{-1}
$$

This is the Bose-Einstein statistics applied to occupation of a phonon mode as a function of temperature. Applying Equation 4.7 to the extracted linewidth at $981 \mathrm{~nm}$ given in Figure 4.14(b), a least-squares fit provides $h v=164.3 \pm 12 \mathrm{~cm}^{-1}$, which corresponds to the energy gap between $E_{01}$ and $E_{00}$.

\subsection{Summary}

It is demonstrated that the confocal luminescent lifetime measurements effectively eliminate the elongation of the measured lifetime owing to radiation trapping. The proposed measurement method allows direct extraction of the luminescence lifetime without any extrapolation procedure or preparation of powdered sample. Besides, it is applicable even to samples with very high $\mathrm{Yb}^{3+}$ concentration up to $\sim 5 \times 10^{21} \mathrm{~cm}^{-3}$, equaling 76 at.\% in $\mathrm{KRE}\left(\mathrm{WO}_{4}\right)_{2}$. Lifetime measurements results obtained from samples with a wide range of $\mathrm{Yb}^{3+}$ concentrations reaffirm that the concentration quenching of lifetime in high $\mathrm{Yb}^{3+}$ concentration $\mathrm{KRE}\left(\mathrm{WO}_{4}\right)_{2}: \mathrm{Yb}^{3+}$ epitaxial layers is rather weak. The lifetime measured from sample with highest $\mathrm{Yb}^{3+}$ concentration of 76 at.\% is 
$222 \mu$ s as compared to the lifetime of $245 \mu$ s obtained from sample with only 1.2 at.\% $\mathrm{Yb}^{3+}$ concentration. In addition, power dependent luminescence decay curves collected from sample with $57 \mathrm{at} \% \mathrm{Yb}^{3+}$ reveal the presence of energy transfer upconversion process which is not typically found in $\mathrm{Yb}^{3+}$-activated materials. The coefficient of ETU process is determined as $1.3 \times 10^{-18} \mathrm{~cm}^{3} / \mathrm{s}$.

Systematic investigation has been conducted to quantify the impact of the polarization disorientation and the stray light on the measured absorbance. The central absorption line near $981 \mathrm{~nm}$ is found to be more sensitive to both factors than other spectral region. The transition cross-sections derived from the absorption measurements on two high $\mathrm{Yb}^{3+}$ concentration epitaxial layers, namely $\mathrm{KLu}_{0.24} \mathrm{Yb}_{0.76}\left(\mathrm{WO}_{4}\right)_{2}$ and $\mathrm{KGd}_{0.43} \mathrm{Yb}_{0.57}\left(\mathrm{WO}_{4}\right)_{2}$, are very similar despite having different doping concentration and substrate. They are also in good agreement to those of the bulk potassium double tungstates, particularly to the $\mathrm{KY}\left(\mathrm{WO}_{4}\right)_{2}: \mathrm{Yb}^{3+}$. The peak absorption and emission crosssections for both samples are $\sim 1.3 \times 10^{-19} \mathrm{~cm}^{2}$ and $\sim 1.6 \times 10^{-19} \mathrm{~cm}^{2}$, respectively, at the central transition line close to $981 \mathrm{~nm}$ wavelength.

The temperature dependent studies reveal that thermal quenching of lifetime is not apparent within the temperature range investigated. Contrarily, the transition crosssections are strongly influenced by the temperature on the sample. With the increase of temperature, the peak cross-sections near $932 \mathrm{~nm}$ and $981 \mathrm{~nm}$ are decreased more rapidly than other spectral regions. With the aid of a simple model, the reduction of peak-absorption cross-section with the increasing temperature can be explained by two effects, which are the reduced fractional population of the relevant Stark level and the linewidth broadening. Further investigation on the magnitude of the extracted linewidths reveals that intra-manifold relaxation within the two lowest Stark levels plays a role in the broadening phenomenon for the central absorption line at $981 \mathrm{~nm}$.

In conclusion, the room temperature spectroscopic findings are favorable for the realization of compact waveguide amplifier. It is experimentally shown that the lifetime quenching is rather limited and the transition cross-sections are preserved in high $\mathrm{Yb}^{3+}$ concentration epitaxial layers. This permits a straightforward approach of enhancing the material gain via the increase of $\mathrm{Yb}^{3+}$ concentration, which in turn allows further miniaturization of waveguide amplifier. Nevertheless, the presence of ETU process may lead to depletion of the population at the excited state and degrade the performance of the amplifier. The temperature dependent studies provide valuable insight on the spectral behavior of a practical device operating at elevated temperature. Though the lifetime of the excited $\mathrm{Yb}^{3+}$ ions is not affected by temperature, the transition crosssections of the major operating wavelengths at $932 \mathrm{~nm}$ and $981 \mathrm{~nm}$ are significantly dependent on the temperature. Such temperature dependency should be taken into account for optimal design of amplifiers or lasers. 



\section{Chapter 5}

\section{Material gain in thin film}

\subsection{Overview}

The pump absorption, signal gain, and luminescence properties in $\operatorname{KRE}\left(\mathrm{WO}_{4}\right)_{2}$ epitaxial layer with high $\mathrm{Yb}^{3+}$ content are investigated with experimental and numerical approaches in this chapter. The objectives of these studies are to evaluate the net gain achievable in the layer and to identify possible causes which deteriorate the gain performance. Amplification scheme with $\sim 932 \mathrm{~nm}$ pump wavelength is adopted, as this allows the use of efficient and high-power InGaAs pump lasers [75] for the eventual waveguide amplifiers. Signal wavelength at $\sim 981 \mathrm{~nm}$ is chosen to leverage the strongest transition line for optical amplification.

Operating heavily $\mathrm{Yb}^{3+}$-doped device under high density of excited ions is known to be challenging and is often inhibited by thermal issues. For instance, additional decay process with high heat generation was observed in $\mathrm{Yb}^{3+}$-doped YAG with high density of excited ions, resulting in limited gain and reduced laser efficiency [151]. Besides, excessive heat generation leading to cease of continuous wave $(\mathrm{CW})$ operation and rapid decline in lasing power [107] was observed from laser based on $\mathrm{KLu}\left(\mathrm{WO}_{4}\right)_{2}:(52$ at.\% $) \mathrm{Yb}^{3+}$ epitaxial layer. Similar observation was reported on laser experiment using $\mathrm{KYb}\left(\mathrm{WO}_{4}\right)_{2}$ crystal, in which short pump pulse duration was needed to minimize the thermal effects [106]. These reports suggest that thermal issue in high $\mathrm{Yb}^{3+}$ concentration $\mathrm{KRE}\left(\mathrm{WO}_{4}\right)_{2}$ devices is not trivial and it should be taken into account during the investigation of material gain.

In this chapter, a theoretical gain model which includes thermal effects is established to study the impact of pump-induced heat generation on material gain under small-signal condition. Temperature-dependent transition cross-sections and thermal conductivities, as well as saturation of pump absorption are considered in the model. Pump-probe optical gain experiment is conducted to determine pump absorption and signal gain in $\mathrm{KGd}_{0.43} \mathrm{Yb}_{0.57}\left(\mathrm{WO}_{4}\right)_{2}$ epitaxial film under intense $\mathrm{CW}$ pumping. By comparing the results determined experimentally and numerically, the factors affecting pump absorption and signal gain are investigated. In addition, near infrared and visible luminescence spectra of the sample are studied to gain further insight on the luminescence behavior under high pump intensity. 


\subsection{Numerical model}

Since $\mathrm{Yb}^{3+}$ ion has only ${ }^{2} \mathrm{~F}_{7 / 2}$ ground state manifold and ${ }^{2} \mathrm{~F}_{5 / 2}$ excited state manifold, a two level system as illustrated in Figure 5.1 is used to model the gain/loss of the optical beams. The model is modified from [28] and it takes into account two distinctive classes of $\mathrm{Yb}^{3+}$ ions, namely active ions and quenched ions. The active ions participate in usual absorption and emission processes. Excited active ions exhibit luminescence lifetime $\tau_{10 a}$ which is measurable using the procedure depicted in previous chapter and the relaxation of active ions to the ground state produces near infrared luminescence. The quenched ions, on the other hand, have a non-radiative lifetime $\tau_{10 q}$ which is significantly shorter than $\tau_{10 a}$. The presence of quenched ions with short excited state lifetime had been reported in various rare-earth-ion-doped materials [28, 152-154]. They are normally not detected from typical lifetime and small-signal absorption measurements but could result in nonsaturable pump absorption, an effect which had been observed in current work and will be further discussed in Section 5.5.

The total density of active/quenched ions residing on the ground state and excited state follows

$$
N_{0 a / q}+N_{1 a / q}=f_{a / q} N_{Y b},
$$

where $N_{0}$ and $N_{1}$ are the respective density of ions at the ground state and excited state while the subscripts $a / q$ indicate active and quenched ions, respectively. $N_{Y b}$ is the density of $\mathrm{Yb}^{3+}$ ions. $f_{a / q}$ represents the fraction of active or quenched ions with a boundary condition of

$$
f_{a}+f_{q}=1 .
$$

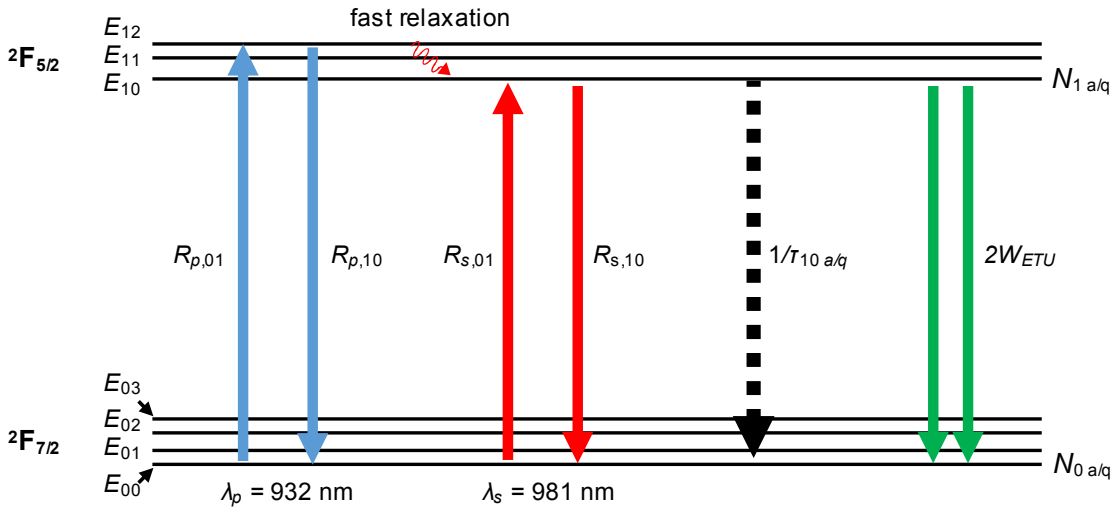

Figure 5.1 Schematic diagram of the two-level system used to model the dynamics of $\mathbf{Y b}^{3+}$ population in the gain medium. 
The rate constants for pump transitions, $R_{p, 01}$ and $R_{p, 10}$, as well as the rate constants for signal transitions, $R_{s, 01}$ and $R_{s, 10}$, are defined by $[127,155]$

$$
\begin{array}{ll}
R_{p, 01}=I_{p} \sigma_{a b s}\left(\lambda_{p}, T\right) \cdot \frac{\lambda_{p}}{h c}, & R_{p, 10}=I_{p} \sigma_{e m}\left(\lambda_{p}, T\right) \cdot \frac{\lambda_{p}}{h c}, \\
R_{s, 01}=I_{s} \sigma_{a b s}\left(\lambda_{s}, T\right) \cdot \frac{\lambda_{s}}{h c}, & R_{s, 10}=I_{s} \sigma_{e m}\left(\lambda_{s}, T\right) \cdot \frac{\lambda_{s}}{h c}
\end{array}
$$

where $I$ represents the optical intensity in $\mathrm{W} / \mathrm{m}^{2}, h$ is Plank's constant and $c$ is the speed of light. $\sigma_{a b s}$ and $\sigma_{e m}$ are the effective absorption and emission cross-sections, respectively, at wavelength $\lambda$ and temperature $T$. The subscripts $p$ and $s$ denote the pump and the signal, respectively. A pump wavelength $\lambda_{p}$ at $932 \mathrm{~nm}$ and a signal wavelength $\lambda_{s}$ at $981 \mathrm{~nm}$ are considered. It is assumed that the excited $\mathrm{Yb}^{3+}$ ions undergo a rapid relaxation from the Stark level with energy $E_{12}$ to the Stark level with the energy $E_{10}$ within a time scale much shorter than the lifetime of the excited state $\tau_{10 \text { a/q }}$.

Assuming that both pump and signal beams possess Gaussian beam profile in the $x-y$ plane, their corresponding intensity profile, $I_{p, s}$ can be modeled with

$$
I_{p, s}=\frac{2 P_{p, s}}{\pi w_{p, s}{ }^{2}} \exp \left[-\frac{2\left(x^{2}+y^{2}\right)}{w_{p, s}{ }^{2}}\right],
$$

where $P$ represents the optical power and $w$ denotes the beam waist.

Apart from pump and signal transitions which are typical for the rate equations of $\mathrm{Yb}^{3+}$-doped materials, the model also includes energy transfer upconversion (ETU) process which had been identified from the pump-dependent luminescence decay curves presented in Chapter 4. Such process involves pairs of $\mathrm{Yb}^{3+}$ ions which are depopulated from the excited state with a rate of decay per unit volume represented by $2 W_{\text {ETU }}\left(N_{1 a / q}\right)^{2}$, where $W_{\text {ETU }}$ is the ETU parameter extracted from the measured luminescence decay curves. Since $\mathrm{Yb}^{3+}$ ion has only two energy levels within the $4 f$ shell, several scenarios could be associated with the ETU process. The combined energy could be released radiatively via emission of photon in visible wavelength range (i.e. cooperative upconversion process). Alternatively, rapid energy migration could occur and energy of pairs of $\mathrm{Yb}^{3+}$ ions could be transferred to impurities. Nevertheless, the exact underlying physical mechanism responsible for the observed ETU process has not been identified at the moment.

The $N, \sigma, I$, and $R$ in Equations 5.1-5.5 are spatially dependent. The $x y z$ discretization for these parameters is taken into account implicitly and their spatial dependent notation will be omitted in subsequent discussion. 


\section{Dynamics of the populations}

The rate equations which govern the change of density of $\mathrm{Yb}^{3+}$ ions at the two energy levels are

$$
\frac{d N_{1 a / q}}{d t}=\left(R_{p, 01}+R_{s, 01}\right) N_{0 a / q}-\left(R_{p, 10}+R_{s, 10}\right) N_{1 a / q}-\frac{N_{1 a / q}}{\tau_{10 a / q}}-2 W_{E T U}\left(N_{1 a / q}\right)^{2},
$$

and

$$
\frac{d N_{0 a / q}}{d t}=-\frac{d N_{1 a / q}}{d t}
$$

Under the condition of $\mathrm{CW}$ pumping, the steady-state condition applies, i.e. $\mathrm{d} N / \mathrm{d} t=0$. Thus, $N_{1}$ can be solved with

$$
N_{1 a / q}=f_{a / q} N_{Y b}-N_{0 a / q}=\frac{-B_{a / q}+\sqrt{{B_{a / q}^{2}-4 A C_{a / q}}^{2 A}}}{2 A}
$$

where,

$$
\begin{gathered}
A=2 W_{E T U}, \\
B_{a / q}=R_{p, 10}+R_{p, 01}+R_{s, 10}+R_{s, 01}+\frac{1}{\tau_{10 a / q}}, \\
C_{a / q}=-f_{a / q} N_{Y b}\left(R_{p, 01}+R_{s, 01}\right) .
\end{gathered}
$$

In the absence of ETU process, $N_{1}$ can be determined from

$$
N_{1 a / q}=\frac{R_{p, 01}+R_{s, 01}}{R_{p, 01}+R_{p, 10}+R_{s, 01}+R_{s, 10}+\tau_{10 a / q}^{-1}} .
$$

The evolution of pump power $P_{p}$ and signal power $P_{s}$ along the propagation direction $z$ is calculated in accordance to

$$
\begin{gathered}
\frac{d P_{p}}{d z}=\left[\sigma_{e m}\left(\lambda_{p}, T\right)\left(N_{1 a}+N_{1 q}\right)-\sigma_{a b s}\left(\lambda_{p}, T\right)\left(N_{0 a}+N_{0 q}\right)\right] P_{p}-\alpha_{p} P_{p}, \\
\frac{d P_{s}}{d z}=\left[\sigma_{e m}\left(\lambda_{s}, T\right)\left(N_{1 a}+N_{1 q}\right)-\sigma_{a b s}\left(\lambda_{s}, T\right)\left(N_{0 a}+N_{0 q}\right)\right] P_{s}-\alpha_{s} P_{s},
\end{gathered}
$$

where $\alpha$ is the propagation loss in the medium. 


\section{Specific case under small-signal condition}

In the event of small-signal amplification, the amplified signal is much weaker than the pump, i.e. $I_{s} \ll I_{p}$, and does not induce appreciable depletion of population from the excited state. Therefore, the respective populations are mainly affected by the dynamics induced by the pump beam. Besides, it is assumed that $\tau_{10 q}$ is infinitesimally small, such that any excited quenched ions would return to the ground state instantaneously. Consequently, the quenched ions would only take part in the absorption of pump and signal photons and $N_{1 q}=0$. Under these circumstances, Equations 5.8-5.12 can be simplified as

$$
\begin{gathered}
N_{1 a}=\frac{-B_{a}+\sqrt{B_{a}^{2}-4 A C_{a}}}{2 A}, \\
A=2 W_{E T U}, \\
B_{a}=R_{p, 10}+R_{p, 01}+\frac{1}{\tau_{10 a}}, \\
C_{a}=-f_{a} N_{Y b} R_{p, 01},
\end{gathered}
$$

for numerical calculation with ETU process, and

$$
N_{1 a}=\frac{R_{p, 01}}{R_{p, 01}+R_{p, 10}+\tau_{10 a}^{-1}},
$$

for numerical calculation without ETU process.

Apart from that, Equations 5.13-5.14 can be expressed as

$$
\begin{gathered}
\frac{d P_{p}}{d z}=\left[\sigma_{e m}\left(\lambda_{p}, T\right)\left(N_{1 a}\right)-\sigma_{a b s}\left(\lambda_{p}, T\right)\left(N_{0 a}+f_{q} N_{Y b}\right)\right] P_{p}-\alpha_{p} P_{p}, \\
\frac{d P_{s}}{d z}=\left[\sigma_{e m}\left(\lambda_{s}, T\right)\left(N_{1 a}\right)-\sigma_{a b s}\left(\lambda_{s}, T\right)\left(N_{0 a}+f_{q} N_{Y b}\right)\right] P_{s}-\alpha_{s} P_{s},
\end{gathered}
$$

The simplification of the equations leads to two direct implications. Firstly, the calculation of pump absorption can be de-coupled from the signal loss/gain calculation, because $N_{1}$ and $N_{0}$ are now dependent only on the pump intensity. As a result, the heat generated in the layer can be calculated by solving the pump absorption and heat generation iteratively. Secondly, the assumption made on $\tau_{10 q}$ eases the calculations involving ETU process because the simplified model contains only one quadratic equation. 


\section{Pump-induced heat generation}

To account for the thermal effect, the spatially dependent heat load in the device is determined by the absorbed pump power per unit volume and the fractional heat load $\eta_{h}$ $[156,157]$

$$
Q_{t h}(x, y, z)=\eta_{h} \alpha_{a b s}(T) \frac{2 P_{p}}{\pi w_{p}^{2}} \exp \left[-\frac{2\left(x^{2}+y^{2}\right)}{w_{p}^{2}}\right] \exp \left[-\alpha_{a b s}(T) z\right]
$$

The fractional heat load $\eta_{h}$ represents the fraction of absorbed pump power which is converted into heat. It consists of the heat load originated from quantum defect $\eta_{Q D}$, ETU process $\eta_{E T U}$, and rapid quenching process $\eta_{q}$,

$$
\eta_{h}=f_{a}\left(\eta_{Q D}+\eta_{E T U}\right)+f_{q} \eta_{q}
$$

The quantum defect is defined by the fractional amount of absorbed photon energy which is converted to heat due to the difference of energy between the absorbed and emitted photons. The head load originated from quantum defect is defined by

$$
\eta_{Q D}=1-\lambda_{p} / \lambda_{m}
$$

where $\lambda_{m}$ is the mean emission wavelength estimated using

$$
\lambda_{m}(T)=\frac{\int \lambda \sigma_{e m}(\lambda, T) d \lambda}{\int \sigma_{e m}(\lambda, T) d \lambda}
$$

Here, the $\lambda_{m}$ is suggested because, in contrarily to a laser, an amplifier operating under small-signal region does not possess a single dominating emission wavelength.

The temperature profile of the sample is governed by the heat conduction equation and the boundary conditions between sample and external environment where heat exchange occur [117],

$$
\begin{gathered}
-k \nabla^{2} T(x, y, z)=Q_{t h}(x, y, z), \\
\frac{\partial T}{\partial n}=h_{T}\left(T_{e x t}-T\right),
\end{gathered}
$$

where $k$ is thermal conductivity, $n$ is the normal to the boundary surface, $h_{T}$ is heat transfer coefficient, and $T_{\text {ext }}$ is external temperature of the thermal contact. 


\section{Solving the equations}

The three dimensional (3D) optical beam and thermal profiles are solved using finite element method with $x y z$ discretization (Comsol Multiphysics 5.1). The flow of calculation is depicted in Figure 5.2. The 3D pump beam and thermal profiles are obtained by solving Eqs. 5.15-5.20 and Eqs. 5.22-5.27 self-consistently. Subsequently, the evolution of the signal along the beam propagation direction is solved with Eqs. 5.15-5.19 and Eq. 5.21 using the pump and temperature profiles computed previously. The thermal lensing effect is not considered in current model. The beam waists are assumed to be constant throughout the layer as the beam waists in the experiment were chosen such that the beams were not strongly diverging within the layer.

As the volume of the sample being pumped (i.e. $V_{p} \approx \pi w_{p}^{2} t_{\text {layer }}$, with $w_{p}<5 \mu \mathrm{m}$, $\left.t_{\text {layer }}=32 \mu \mathrm{m}\right)$ is much smaller than the sample's volume $(\sim 10 \mathrm{~mm} \times 10 \mathrm{~mm} \times 1 \mathrm{~mm})$, the modeled sample is partitioned into sections with different mesh size, see Figure 5.3. A fine mesh with a maximum and minimum element sizes of $w_{p} / 5$ and $0.005 \mu \mathrm{m}$ is used at the pump section (within $2 \times w_{p}$ in radial direction), whereas coarser meshes are used in other sections. Only a quarter of the sample is modelled to leverage the symmetry property of the sample.

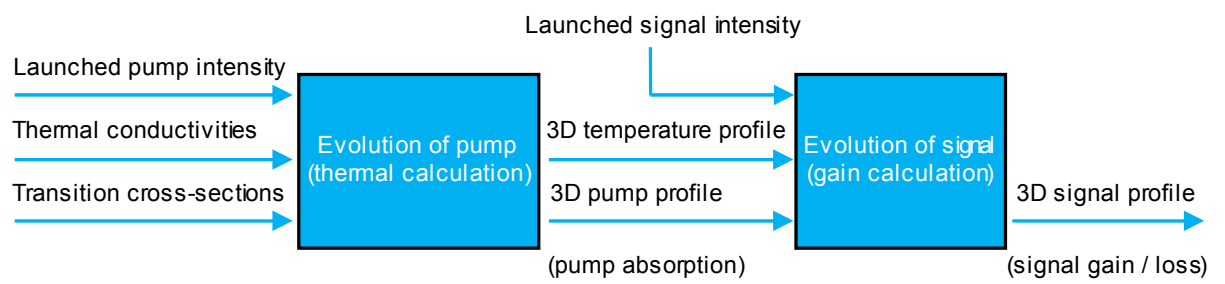

Figure 5.2 Schematic diagram indicating the flow of numerical calculation. The pump beam and thermal profiles are solved with $x, y$, and $z$ discretization self-consistently. Next, the three dimensional signal beam profile is solved using the pump beam and thermal profiles. The pump absorption and signal gain/loss at each launched pump power value are extracted from the calculated beam profiles.
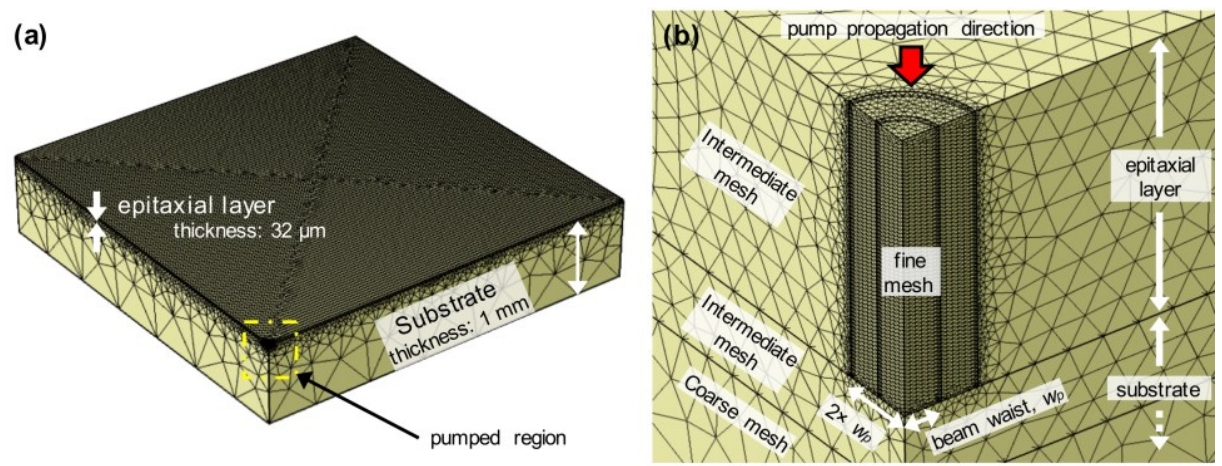

Figure 5.3 Mesh setup for numerical calculation. (a) shows the mesh grid of a quarter of the sample. (b) shows the close-up view near the pumped region. The finest mesh is used for region covering $2 \times$ beam waist in radial direction. Intermediate spacing mesh is used for region covering $10 \times$ beam waist and a coarse mesh is used for other region to reduce the time needed for numerical calculation. 


\subsection{Details of sample and material parameters}

The high $\mathrm{Yb}^{3+}$ concentration sample used for the investigation of material gain consists of $32 \mu \mathrm{m}$ thick $\mathrm{KGd}_{0.43} \mathrm{Yb}_{0.57}\left(\mathrm{WO}_{4}\right)_{2}$ layer grown on undoped $\mathrm{KY}\left(\mathrm{WO}_{4}\right)_{2}$ substrate. Relevant parameters of the epitaxial layer and the substrate which are used in numerical calculations are summarized in Table 5.1.

For the ease of numerical calculation, a generic empirical formula adapted from [85] is used here for all temperature-dependent parameters,

$$
X(T)=X\left(T_{0}\right)\left(1+c_{1} T+c_{2} T^{2}+c_{3} T^{3}\right)
$$

where $X$ is the parameter of interest and $T_{0}$ is an arbitrary reference temperature. $c_{1}, c_{2}$, and $c_{3}$ are fitted parameters.

\section{Spectroscopic parameters}

The transition cross-sections and lifetime of the sample which had been reported in Chapter 4 are used for numerical calculation. The effective transition cross-section data determined at $E \| N_{m}$ at the pump and signal wavelengths under various temperature settings are plotted in Figure 5.4(a) as symbols. The corresponding fitted curves using Eq. 5.28 are displayed as lines in Figure 5.4(a). The relevant fitted parameters can be found in Table 5.2. Constant lifetime value measured using the confocal setup is used, i.e. $\tau_{10 a}=228 \mu \mathrm{s}$, as the lifetime has no significant dependence on sample's temperature. ETU parameter $W_{E T U}$ of $1.3 \times 10^{-18} \mathrm{~cm}^{3} / \mathrm{s}$ extracted from the powerdependent luminescence decay curves is adopted.
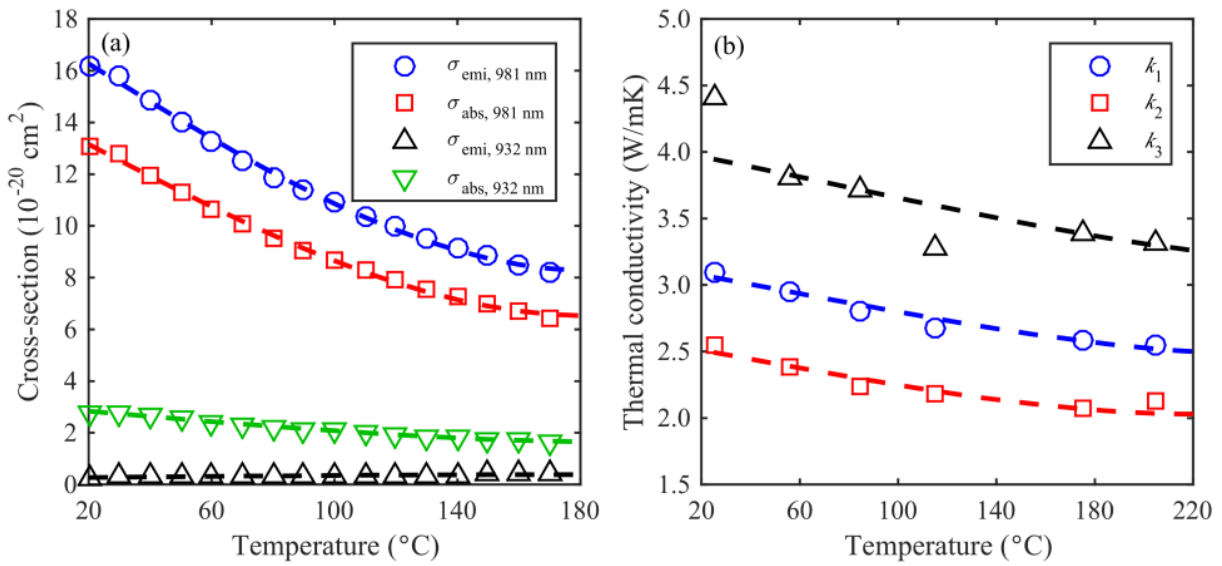

Figure 5.4 Temperature dependent data of (a) effective transition cross-sections at $E|| N_{m}$ extracted from Chapter 4, and (b) thermal conductivities $k_{1}, k_{2}$, and $k_{3}$ for the respective thermal axis of $X_{1}^{\prime}$, $X_{2}{ }_{2}$, and $X_{{ }_{3}}$ (data is taken from [116]). The lines are plotted with Eq. 5.28 using the least-square fitted parameters listed in Table 5.2 and Table 5.3. 
Table 5.1 Details of the relevant temperature-independent input parameters used for numerical modeling.

\begin{tabular}{|l|c|}
\hline \multicolumn{1}{|c|}{ Parameter } & Value \\
\hline Material composition & $\mathrm{KGd}_{0.43} \mathrm{Yb}_{0.57}\left(\mathrm{WO}_{4}\right)_{2}$ \\
\hline Thickness of polished layer, $t_{\text {layer }}$ & $32 \mu \mathrm{m}$ \\
\hline $\mathrm{Yb}^{3+}$ concentration, $N_{Y b}$ & $3.8 \times 10^{21} \mathrm{~cm}^{-3}$ \\
\hline Substrate & $\mathrm{KY}\left(\mathrm{WO}_{4}\right)_{2}$ \\
\hline Thickness of substrate, $t_{\text {substrate }}$ & $\sim 1 \mathrm{~mm}$ \\
\hline Width of substrate, $w_{\text {substrate }}$ & $\sim 10 \mathrm{~cm}$ \\
\hline Depth of substrate, $d_{\text {substrate }}$ & $\sim 10 \mathrm{~cm}$ \\
\hline Pump beam's radius, $w_{p}$ & $4.75 \mu \mathrm{m}$ \\
\hline Signal beam's radius, $w_{s}$ & $2.5 \mu \mathrm{m}$ \\
\hline Pump wavelength, $\lambda_{p}$ & $932 \mathrm{~nm}$ \\
\hline Signal wavelength, $\lambda_{s}$ & $981 \mathrm{~nm}$ \\
\hline Mean emission wavelength, $\lambda_{m}$ & $996 \mathrm{~nm}$ \\
\hline Propagation losses, $\alpha_{p} \& \alpha_{s}$ & 0 \\
\hline Measured lifetime, $\tau_{10}$ & $228 \mu \mathrm{s}$ \\
\hline Energy transfer upconversion parameter, $W_{E T U}$ & $1.3 \times 10^{-18} \mathrm{~cm}^{3} \mathrm{~s}^{-1}$ \\
\hline Fraction of quenched ions, $f_{q}$ & $0 \mathrm{or} 0.17$ \\
\hline Heat transfer coefficient, $h_{T}$ & $20 \mathrm{Wm}^{-2} \mathrm{~K}^{-1}$ \\
\hline External temperature, $T_{\text {ext }}$ & $300 \mathrm{~K}$ \\
\hline
\end{tabular}

\section{Thermal related parameters}

The temperature-dependent thermal conductivities reported for $\mathrm{Yb}^{3+}$-doped $\mathrm{KLu}\left(\mathrm{WO}_{4}\right)_{2}$ [116] are used here as there is a lack of comprehensive temperature-dependent data for other double tungstates. Figure 5.4(b) shows the thermal conductivities $k_{1}, k_{2}$, and $k_{3}$ for the respective thermal axis of $X_{1}, X_{2}$, and $X_{3}$. The thermal axis $X_{2}$ coincides with $N_{p}$ optical axis and they are parallel to the direction of beam propagation in the gain experiment. The relevant fitted values are provided in Table 5.3.

The external temperature $T_{\text {ext }}$ is assumed to be $300 \mathrm{~K}$ as no active cooling was applied to the sample. The value of heat transfer coefficient $h_{T}$ is highly dependent on the thermal interfaces of the sample during the experiment and it is typically regarded as adjustable parameter for thermal calculations. Typical $h_{T}$ value accounting for the surface radiation and convection between the air/sample interfaces is $\sim 10 \mathrm{Wm}^{-2} \mathrm{~K}^{-1}$ for each of the two cases $[158,159]$, whereas $h_{T}$ value for a sample in bare contact with water-cooled copper holder can be as high as $2500 \mathrm{Wm}^{-2} \mathrm{~K}^{-1}$ [160]. Since only a small part of the $\mathrm{KGd}_{0.43} \mathrm{Yb}_{0.57}\left(\mathrm{WO}_{4}\right)_{2}$ sample was clamped by two small copper plates during the measurements (see Figure 5.5) and no special effort was applied to improve the thermal contact between the sample and holder, a rather conservative $h_{T}$ value of $20 \mathrm{Wm}^{-2} \mathrm{~K}^{-1}$ is employed for all surfaces of the sample in the numerical calculation. 
The mean emission wavelength $\lambda_{m}$ calculated from the temperature dependent $\sigma_{e m}$ spectra using Eq. 5.25 is $996.14 \pm 1.3 \mathrm{~nm}$ throughout $20-170{ }^{\circ} \mathrm{C}$, which is in good agreement with the average fluorescence wavelength of $997 \mathrm{~nm}$ as reported in [77]. There could be a small dependence of $\lambda_{m}$ on temperature since $\sigma_{e m}$ beyond $1050 \mathrm{~nm}$ wavelength is associated with high uncertainty and not considered in the calculation. Consequently, the fractional heat load due to quantum defect $\eta_{Q D}$ is calculated as $6.4 \%$. The total energy associated with the quenched ions is expected to result in generation of phonons, hence $\eta_{q}$ is $100 \%$. It is assumed that the heat load generated by the ETU process is equal to $\eta_{Q D}$, i.e. $\eta_{E T U}=\eta_{Q D}$, due the uncertain nature of the ETU process. In any case, any assumption which results in higher $\eta_{E T U}$ can be balanced by accordingly higher $h_{T}$ because there are high uncertainties associated with both parameters. With these considerations, the fractional heat load used for the numerical calculation is

$$
\eta_{h}=f_{a}(6.4 \%)+f_{q}(100 \%) .
$$

\section{Other parameters}

The radii of the focused pump beam $w_{p}$ and signal beam $w_{s}$ listed in Table 5.1 are based on measured beam profiles. To ensure optimum pump-signal overlapping, the beam sizes in the experiment were chosen to be as small as possible while ensuring that their confocal lengths (i.e. twice of Rayleigh length $z_{R}$ ) are longer than the layer thickness. The use of constant $w$ can be justified with an example by considering $w=2.5 \mu \mathrm{m}$ at $\lambda=981 \mathrm{~nm}$, in which the $z_{R}$ within the sample with refractive index of $\sim 2.0$ is $\sim 40 \mu \mathrm{m}$. Assuming that the focused beam is located at the center of the layer, the maximum beam waist within the $32 \mu \mathrm{m}$ thick layer would be $\sim 3.2 \mu \mathrm{m}$. The deviation of the beam waist is comparable to the measurement uncertainty of $\sim 0.5 \mu \mathrm{m}$ from the beam profiler. In addition, as the propagation loss for devices made of similar material at $\sim 1000 \mathrm{~nm}$ wavelength is typically $<0.04 \mathrm{~dB} / \mathrm{cm}$ [99], both $\alpha_{p}$ and $\alpha_{s}$ are considered to be negligible as compared to the dynamics attributed to the active ions, i.e. $\alpha_{p}=\alpha_{s}=0$.

Table 5.2 Fitted parameters for the effective transition cross-sections used in numerical modeling.

\begin{tabular}{|c|c|c|c|c|c|}
\hline Effective cross-section ${ }^{*}$ & $T_{0}(\mathbf{K})$ & $\sigma\left(T_{0}\right)\left(10^{-20} \mathrm{~cm}^{2}\right)$ & $c_{1}\left(10^{-3} \mathrm{~K}^{-1}\right)$ & $c_{2}\left(10^{-5} \mathrm{~K}^{-2}\right)$ & $c_{3}\left(10^{-8} \mathrm{~K}^{-3}\right)$ \\
\hline$\sigma_{a b s, 932 \mathrm{~nm}}$ & 293.15 & 2.81 & 7.063 & -3.574 & 4.004 \\
\hline$\sigma_{e m, 932 \mathrm{~nm}}$ & 293.15 & 0.267 & -7.527 & 3.925 & -4.512 \\
\hline$\sigma_{a b s, 981 \mathrm{~nm}}$ & 293.15 & 13.1 & 9.598 & -4.933 & 5.672 \\
\hline$\sigma_{e m, 981 \mathrm{~nm}}$ & 293.15 & 16.2 & 9.301 & -4.775 & 5.482 \\
\hline
\end{tabular}

${ }^{*}$ Applicable for temperature range within $293.15-443.15 \mathrm{~K}$.

Table 5.3 Fitted parameters for the thermal conductivities used in numerical modeling.

\begin{tabular}{|c|c|c|ccc|}
\hline Thermal conductivity $^{* *}$ & $\boldsymbol{T}_{\boldsymbol{0}}(\mathbf{K})$ & $\boldsymbol{k}\left(\boldsymbol{T}_{\boldsymbol{0}}\right)\left(\mathbf{W m}^{-\mathbf{1}} \mathbf{K}^{\mathbf{- 1}}\right)$ & $\boldsymbol{c}_{\boldsymbol{1}}\left(\mathbf{1 0}^{\mathbf{- 3}} \mathbf{K}^{\mathbf{- 1}}\right)$ & $\boldsymbol{c}_{\boldsymbol{2}}\left(\mathbf{1 0}^{-\mathbf{5}} \mathbf{K}^{\mathbf{2}}\right)$ & $\boldsymbol{c}_{\mathbf{3}}\left(\mathbf{1 0}^{\mathbf{- 8}} \mathbf{K}^{-\mathbf{3}}\right)$ \\
\hline$k_{1}$ & 298.15 & 3.09 & 1.851 & -0.9055 & 0.9155 \\
$k_{2}$ & 298.15 & 2.55 & 2.298 & -1.170 & 1.257 \\
$k_{3}$ & 298.15 & 4.00 & 1.402 & -0.6786 & 0.6439 \\
\hline
\end{tabular}

${ }^{* *}$ Applicable for temperature range within $293.15-493.15 \mathrm{~K}$. 


\subsection{Measurement setup}

The pump-probe setup used for optical gain measurement is depicted in Figure 5.5. A CW Ti:Sapphire laser (Spectra-Physics 3900S) tuned to $932 \mathrm{~nm}$ wavelength is used as the pump source. The pump power is controlled using a half-wave plate and a Glan polarizer. The signal beam at $\sim 981 \mathrm{~nm}$ wavelength with a bandwidth $\leq 1 \mathrm{~nm}$ is obtained with a supercontinuum light source (Fianium SC-450) passing through a monochromator. It is mechanically chopped at $233 \mathrm{~Hz}$ with $50 \%$ duty cycle for lock-in detection to discriminate the detected signal from spontaneous emission produced by the sample and from different background noise sources. A low launched signal power $<100 \mathrm{nW}$ is used to ensure a signal amplification within the small-signal-gain regime.

Both pump and signal beams are combined using a dichroic mirror and subsequently focused perpendicularly on the sample at $\mathrm{E} \| N_{m}$ polarization using a microscope objective (MO). The residual pump and signal beams are collected using another MO with numerical aperture higher than the incident MO. In order to ensure a good overlapping of the pump and signal beams, the foci are scanned using a beam profiler (Thorlabs BP209) positioned on a motorized linear stage and the beam expander optics are adjusted for optimal beam overlapping prior to the experiment.

The pump and signal beams passing through the sample are directed to a light guide which is connected to a spectrometer (Jobin Yvon iHR550) equipped with a pump filter and a cooled InGaAs detector. The combination of the pump filter and the dispersive grating in the spectrometer ensures that the residual pump power is much lower than the amplified signal power. The pump power is measured using a thermopile (Coherent FieldMaxII, PM10) before the incident MO and after the collection MO. The launched and absorbed pump powers are deduced after correcting for the objectives' transmittance and the Fresnel reflections on the sample. No special thermal management was applied on the samples during the measurement.

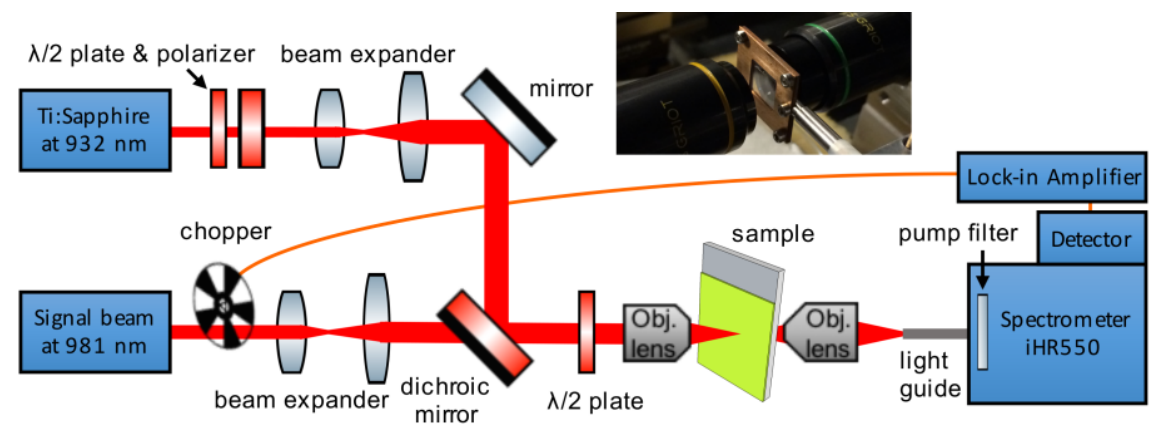

Figure 5.5 Schematic drawing of the pump-probe gain measurement setup. The optical beams are launched perpendicularly to the sample. The signal beam is obtained with a supercontinuum light source passing through a monochromator. Inset shows the sample mounted on a holder made of thin copper plates with clear aperture of $\sim 8.6 \mathrm{~mm} \times \sim 8.6 \mathrm{~mm}$. 


\subsection{Results}

\section{Pump absorption}

The heat generation in the sample is highly dependent on the pump absorption. As such, the pump absorption characteristic of the $\mathrm{KGd}_{0.43} \mathrm{Yb}_{0.57}\left(\mathrm{WO}_{4}\right)_{2}$ epitaxial layer is investigated by comparing the experiment and numerical results. The measured data points of pump absorption versus launched pump power are plotted in Figure 5.6 as square boxes. The pump absorption is defined as

$$
A_{p}=-10 \log _{10}\left[P_{p}\left(t_{\text {layer }}\right) / P_{p}(0)\right],
$$

where $P_{p}(0)$ is the launched pump power at front surface of the epitaxial layer and $P_{p}\left(t_{\text {layer }}\right)$ is the residual pump power at rear surface of the epitaxial layer. The calculated pump absorption using different models are shown as lines in Figure 5.6. These results take into account the pump-induced heating based on the fractional heat load defined by Eq. 5.29 as well as the changes of transition cross-sections and thermal conductivities due to the heat generated within the layer.

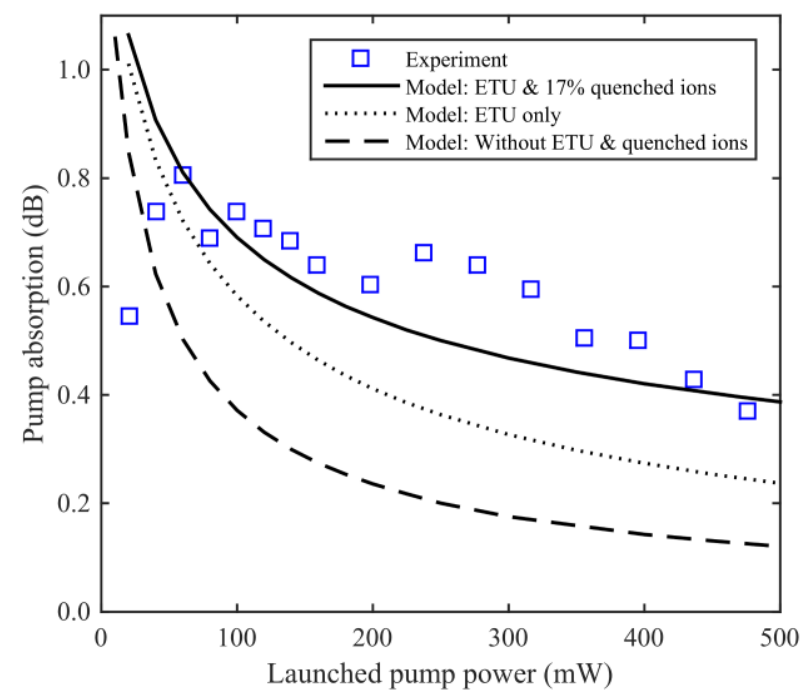

Figure 5.6 Pump absorption versus launched pump power for $\operatorname{KGd}_{0.43} \mathrm{Yb}_{0.57}\left(\mathrm{WO}_{4}\right)_{2}$ epitaxial layer. The experiment data is shown as squares. Dash line represents the modeled result without considering ETU process and quenched ions (i.e. $W_{E T U}=0$ and $f_{q}=0$ ). Dotted line shows the modeled result which accounts for the ETU process but not the quenched ions (i.e. $W_{E T U}=1.3 \times 10^{-18} \mathrm{~cm}^{3} \mathrm{~s}^{-1}$ and $f_{q}=0$ ). The solid line is calculated with the model which considers both ETU process as well as $17 \%$ of quenched ions $\left(W_{E T U}=1.3 \times 10^{-18} \mathrm{~cm}^{3} \mathrm{~s}^{-1}\right.$ and $\left.f_{q}=0.17\right)$. All calculated results take into account the pump-induced heat generation with the fractional heat load given by Eq. 5.29. 
The numerical result calculated using typical model for $\mathrm{Yb}^{3+}$-doped devices, i.e. without both ETU process and quenched ions (dash line in Figure 5.6), show a more intense saturation of pump absorption as compared to the experimental results. The numerical result indicates that bleaching of ground state population occurs due to high pump intensity which in turn results in low pump absorption at high launched pump power. The ETU process serves as additional decay channel which depopulates the excited state. Therefore, the pump absorption calculated with the consideration of ETU process (dotted line) is increased because significant amount of ions is available at the ground state even though the pump intensity is high. Nevertheless, there is still a rather large discrepancy between the experimental and numerical pump absorption results even if the ETU process has been taken into account. The nonsaturable pump absorption is addressed by considering that $17 \%$ of the $\mathrm{Yb}^{3+}$ ions are rapidly quenched in addition to the ETU process. The pump absorption result calculated with the consideration of these two factors is shown as solid line in Figure 5.6 and it is in reasonable agreement with the experiment results. The fluctuations of the experiment data as compared to the calculated curve is likely due to the relatively high measurement uncertainty (estimated as $\pm 0.17 \mathrm{~dB}$ by considering uncertainty of $2 \%{ }^{*}$ on the measured pump power) as compared to the magnitude of absorbed pump power.

\section{Temperature profile}

The influence of ETU process and quenched ions on the temperature of the layer is examined by comparing the average temperature within the pumped volume ( $V_{p} \approx \pi w_{p}{ }^{2} t_{\text {layer }}$ ) calculated from each model as shown in Figure 5.7. The average temperature within the pumped volume $T_{p}$ calculated from the model without ETU process and quenched ions (dash line in Figure 5.7) is $\sim 30.2^{\circ} \mathrm{C}$ at launched pump power of $500 \mathrm{~mW}$. This is primarily due to the low amount of calculated absorbed pump power which deviates significantly from experimental data as observed in Figure 5.6 and low fractional heat load $\eta_{Q D}$ of $6.4 \%$. With the inclusion of ETU process, the calculated $T_{p}$ (dotted line in Figure 5.7) is increased to $\sim 33.65^{\circ} \mathrm{C}$ at launched pump power of $500 \mathrm{~mW}$. Since it is assumed that $\eta_{E T U}=\eta_{Q D}$, the increase of temperature with the inclusion of ETU process is caused by the increase of calculated pump absorption from $0.12 \mathrm{~dB}$ to $0.24 \mathrm{~dB}$. Nevertheless, the comparison of the modeled results calculated with and without ETU process only provides a qualitative overview as the actual $\eta_{E T U}$ could be significantly larger than the value assumed. By considering both ETU process and quenched ions, the total fractional heat load $\eta_{h}$ is substantially

\footnotetext{
${ }^{*} 2 \%$ of measurement uncertainty is estimated based on the specification sheet of FieldMax II detector, where each of the meter and sensor head contributes $1 \%$ of measurement uncertainty under the worst case scenario. The uncertainty in absorbed pump power $(\mathrm{dB})$ is estimated with $-10 \log _{10}\left\{\left[P_{p}\left(t_{\text {layer }}\right) \times 1.02\right] /\left[P_{p}(0) \times 0.98\right]\right\}$ which is equivalent to $-10 \log _{10}\left[P_{p}\left(t_{\text {layer }}\right) / P_{p}(0)\right]-10 \log _{10}[1.02 / 0.98]$ or $A_{p}-0.1737 \mathrm{~dB}$.
} 
increased to $22.3 \%$. The calculated $T_{p}$ using the model with ETU and quenched ions (solid line in Figure 5.7) is increased significantly to $\sim 66.5^{\circ} \mathrm{C}$ at launched pump power of $500 \mathrm{~mW}$.

The three dimensional isothermal contours calculated using the model with ETU and quenched ions at launched pump power of $500 \mathrm{~mW}$ is shown in Figure 5.8(a). The temperature profiles along the beam propagation direction calculated at various pump power values are depicted in Figure 5.8(b). The highest temperature is observed at the pump incident surface, which is typical for end-pumped devices. This is mainly due to the high pump power at the incident surface. The amount of heat generated decreases along the propagation direction as part of the pump power is absorbed by the $\mathrm{Yb}^{3+}$ ions. Besides, as the heat generation process ceases at the substrate, the substrate effectively eases the dissipation of heat near the epitaxial layer/substrate interface. Therefore, inflection point is observed at the epitaxial layer/substrate interface for all the curves. Figure 5.8(c) shows the thermal profile on the pump incident surface along the radial direction at various launched pump power. As expected from Equation 5.26, the center axis exhibits the highest temperature as it is the heat generation region and the temperature reduces rapidly with the increasing distance from the axis. The temperature profile along $X_{3}{ }_{3}$ axis resembles the trend along $X^{\prime}{ }_{1}$ except that the temperature at the region near the center axis in $X^{\prime}{ }_{3}$ direction is slightly lower due to higher thermal conductivity along $X^{\prime}{ }_{3}$.

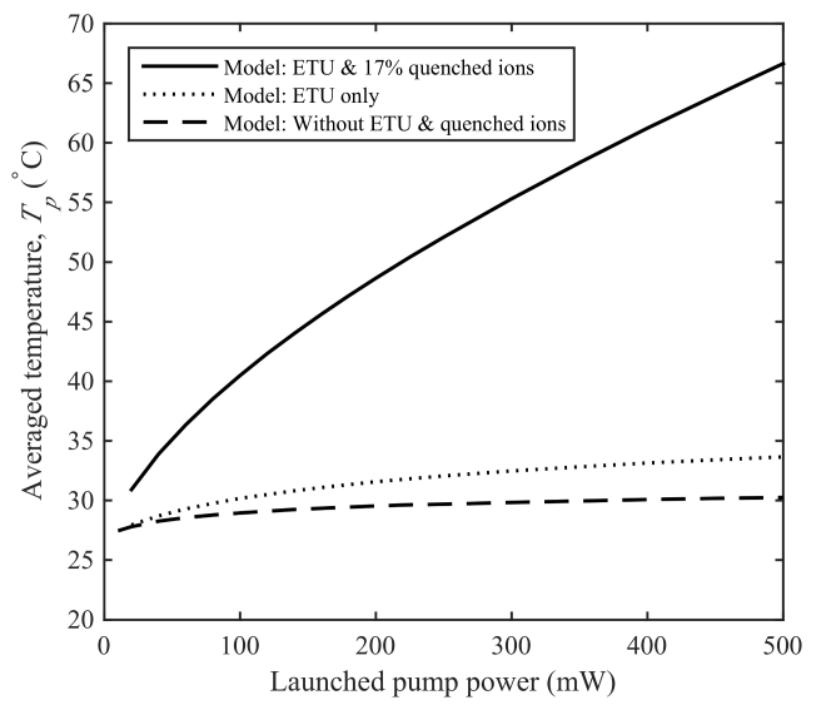

Figure 5.7 Averaged temperature within the pumped volume $T_{p}$ versus launched pump power calculated using different models. 

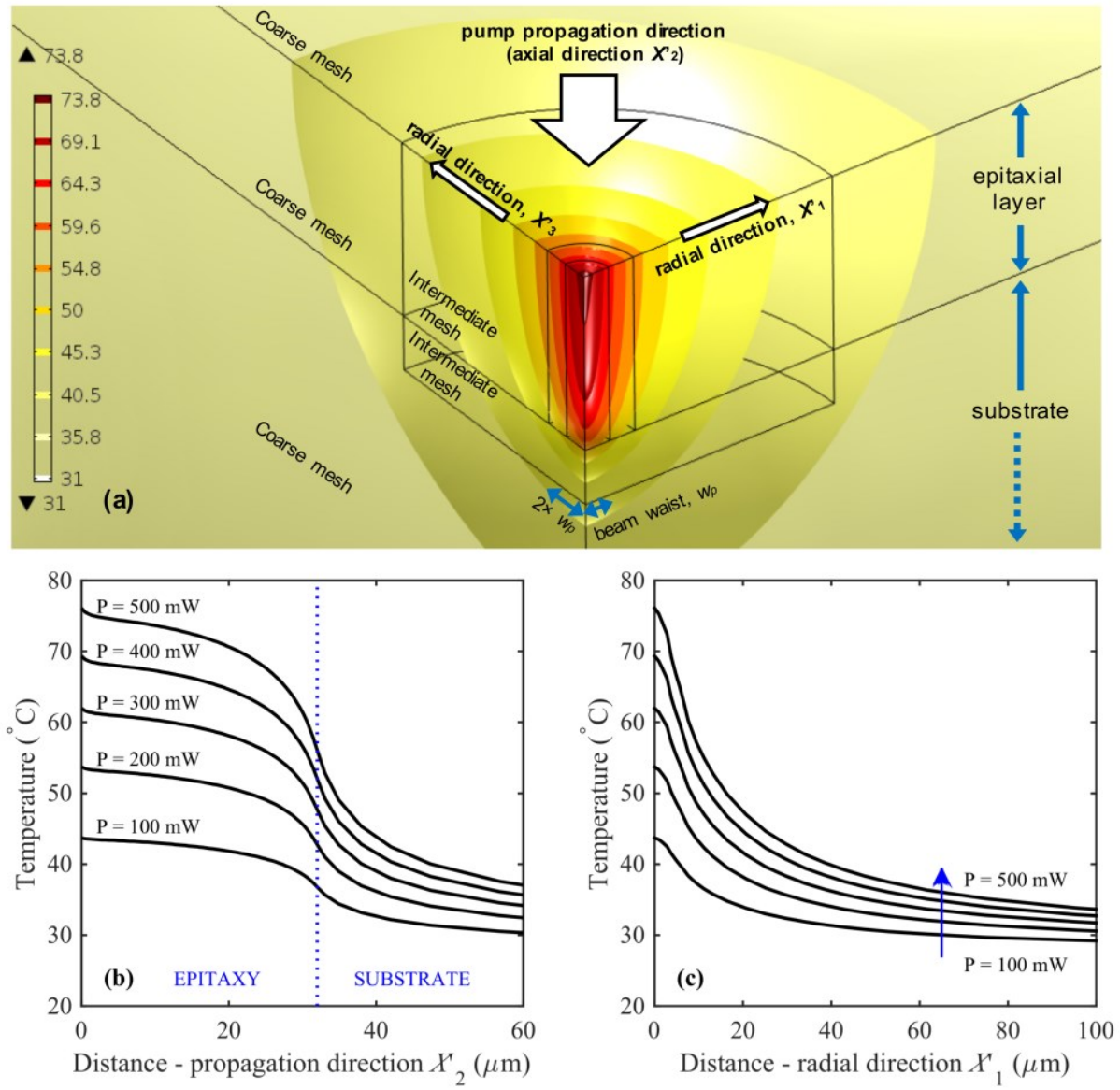

Figure 5.8 (a) Three-dimensional isothermal contours calculated using the model with ETU process and $17 \%$ quenched ions at $500 \mathrm{~mW}$ launched pump power. (b) Temperature profile along the pump propagation direction ( $X^{\prime}{ }_{2}$ axis). (c) Temperature profile on the pump incident surface along $X_{1}^{\prime}$ axis.

\section{Optical gain}

During the pump-probe experiment, the signal intensity is recorded when the pump is launched $\left(I_{o n}\right)$ and when the pump is blocked $\left(I_{o f f}\right)$. The net gain of the samples $G$ is determined using the expression

$$
G=10 \log _{10}\left(I_{\text {on }} / I_{\text {off }}\right)-\alpha_{\text {total }} \cdot t_{\text {layer }},
$$

where $\alpha_{\text {total }}$ is the total loss per unit length for the signal

$$
\alpha_{\text {total }}=\alpha_{s}+4.343 \cdot \sigma_{a b s}\left(\lambda_{s}, T\right) \cdot N_{Y b} \text {. }
$$

The propagation loss $\alpha_{s}$ is $<0.04 \mathrm{~dB} / \mathrm{cm}$ [99], hence it is negligible as compared to the absorption loss due to $\mathrm{Yb}^{3+}$ ions in the epitaxial layer, $4.343 \times \sigma_{a b s}\left(\lambda_{s}, T\right) \times N_{Y b}$. During the experiment, the $I_{o n}$ and $I_{\text {off }}$ are recorded interchangeably at each launched pump power 
value. Therefore, it is assumed that the pump-induced heating persists as the dissipation of heat would take place in a relatively longer time scale. Consequently, the absorption cross-section values calculated using $T_{p}$ shown as solid curve in Figure 5.7 are used to evaluate the net gain.

The net gain results are shown in Figure 5.9(a). The maximum signal enhancement, i.e. $10 \log _{10}\left(I_{o n} / I_{\text {off }}\right)$, measured at $\sim 475 \mathrm{~mW}$ launched pump power is $8.129 \mathrm{~dB}$. Considering the absorption cross section of $1.044 \times 10^{-19} \mathrm{~cm}^{2}$ calculated at the estimated $T_{p}$ of $65.4{ }^{\circ} \mathrm{C}$, the calculated total absorption is $5.513 \mathrm{~dB}$. Therefore, an internal net gain of $2.616 \mathrm{~dB}$, or $817 \mathrm{~dB} / \mathrm{cm}$, is obtained. The modeled gain result with the consideration of ETU process and $17 \%$ quenched ions (solid line) is in reasonable agreement to the results calculated based on experiment signal enhancement data.

The impact of ETU process and quenched ions on the attainable gain is shown in Figure 5.9(b). Without both ETU process and quenched ions, the modeled gain is $2035 \mathrm{~dB} / \mathrm{cm}$ for $500 \mathrm{~mW}$ launched pump power (dash line). The ETU process causes depletion of excited state's population. By including this process in the model, the threshold pump power required for signal transparency (i.e. gain/loss $=0$ ) is increased from $\sim 15.5 \mathrm{~mW}$ to $\sim 31.5 \mathrm{~mW}$ and the maximum gain is reduced to $1760 \mathrm{~dB} / \mathrm{cm}$ (dotted line). The assumption on the presence of quenched ions which is equivalent to $17 \%$ of the total ions implies that only the remaining $83 \%$ of active ions are participating in the optical amplification process. Besides, the substantial increase of heating causes reduction of transition cross-sections with the increase of pump power. As a result, the modeled gain with the consideration of both ETU process and quenched ions (solid line) is effectively clamped to $\sim 850 \mathrm{~dB} / \mathrm{cm}$ at $500 \mathrm{~mW}$ launched pump power.
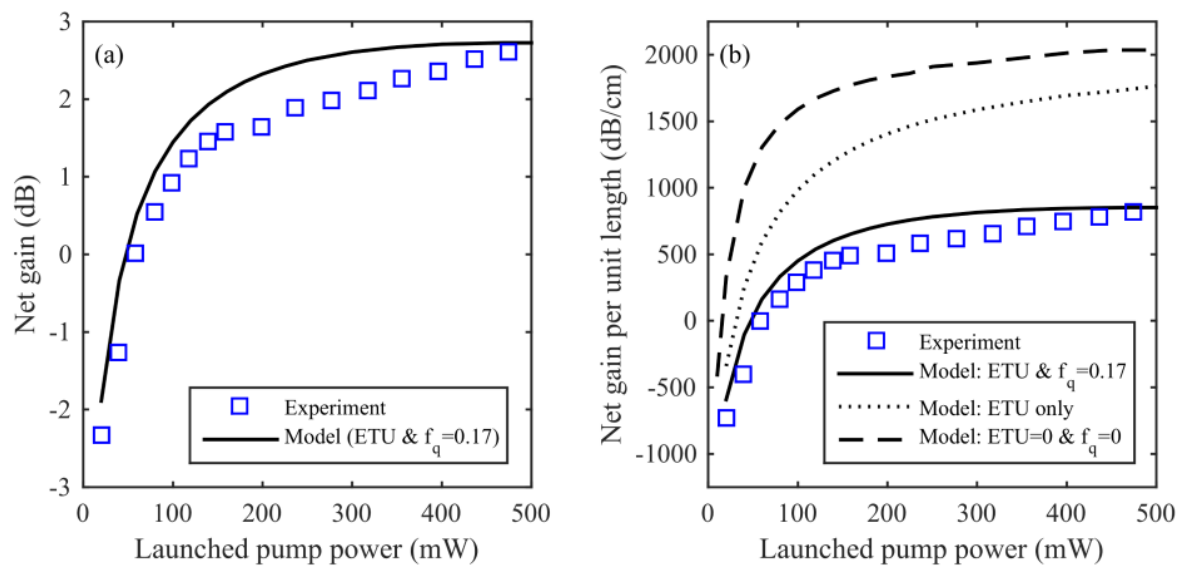

Figure 5.9 (a) Net gain results for $\mathrm{KGd}_{0.43} \mathrm{Yb}_{0.57}\left(\mathrm{WO}_{4}\right)_{2}$. (b) shows the corresponding net gain per unit length results calculated from (i) solid line: model with both ETU process and quenched ions of 17\%, (ii) dotted line: model with only ETU process, and (iii) dash line: model without both ETU process and quenched ions. 


\section{Luminescence spectra}

The $\mathrm{KGd}_{0.43} \mathrm{Yb}_{0.57}\left(\mathrm{WO}_{4}\right)_{2}$ sample did not show any measurable absorption feature from other active ions or $\mathrm{Yb}^{2+}$ within visible wavelength range* during the investigation of absorption using spectrophotometer. However, bluish emission was observed during the gain experiment under high pump intensity, which is likely due to emission from the impurities. In order to gather further insight on the sample's behavior at high pump intensity, the luminescence from the sample was recorded in a separate experiment using the same setup shown in Figure 5.5 by focusing only the pump beam at $932 \mathrm{~nm}$ wavelength perpendicularly to the sample and collecting the luminescence of the sample from the other end for detection via the spectrometer. The near infrared (NIR) spectra were recorded with cooled InGaAs detector whereas the visible spectra were measured with photomultiplier. A longwave-pass pump filter with cutoff wavelength at $950 \mathrm{~nm}$ was used for NIR luminescence measurement and a bandpass filter with $\geq 80 \%$ transmission within 345-615 $\mathrm{nm}$ wavelength range was used for visible luminescence measurement. The recorded NIR spectra were corrected for spectral response of the detection system determined using a calibration lamp (Ocean Optics LS-1-CAL).

Figure 5.10 shows the corrected NIR emission spectra at various launched pump power values (denoted as $P_{l}$ hereafter). Three emission peaks are observed at $\sim 981 \mathrm{~nm}$, $\sim 1000 \mathrm{~nm}$, and $\sim 1023 \mathrm{~nm}$ wavelengths, which correspond to the $E_{10} \rightarrow E_{00}, E_{10} \rightarrow E_{01}$, and $E_{10} \rightarrow E_{02}$ transitions, respectively. The emission spectra are more intense with the increase of $P_{l}$ up to $\sim 120 \mathrm{~mW}$ (blue curves in Figure 5.10). However, further increase of $P_{l}$ leads to reduced intensity in the measured spectra. In the case of $P_{l} \approx 280 \mathrm{~mW}$, the intensity at the dips near $\sim 990 \mathrm{~nm}$ and $\sim 1015 \mathrm{~nm}$ wavelengths is higher than the spectrum measured at $P_{l} \approx 120 \mathrm{~mW}$. At high $P_{l}$ of $\sim 480 \mathrm{~mW}$, a smoothened emission spectrum is observed and the emission peaks at $\sim 1000 \mathrm{~nm}$ and $\sim 1023 \mathrm{~nm}$ wavelengths become less distinguishable as compared to the emission spectra measured at lower $P_{l}$.

The intensities of the emission peaks at $\sim 981 \mathrm{~nm}, \sim 1000 \mathrm{~nm}$, and $\sim 1023 \mathrm{~nm}$ are extracted from the spectra and the results are depicted in Figure 5.11(a). These emission peaks evolve with similar trend with respect to $P_{l}$. A rapid increase of intensity occurs when the pump power is low and the highest intensity is observed at $P_{l} \approx 120 \mathrm{~mW}$. As $P_{l}$ is increased beyond $120 \mathrm{~mW}$, the intensity decreases monotonously. Apart from the analysis on the intensity of the peaks, multiple peaks fitting was performed on the measured spectra by assuming three Lorentzian peaks using data analysis program (Origin 9.1). The extracted full-width-at-half-maximum (FWHM) values for the peak at $\sim 981 \mathrm{~nm}$ wavelength for different $P_{l}$ values are plotted in Figure 5.11(b) and significant increase of FWHM is evident over the entire $P_{l}$ range investigated.

\footnotetext{
${ }^{*} \mathrm{Yb}^{2+}$ in YAG exhibits two smooth absorption bands around $400 \mathrm{~nm}$ and $600 \mathrm{~nm}$ [140].
} 

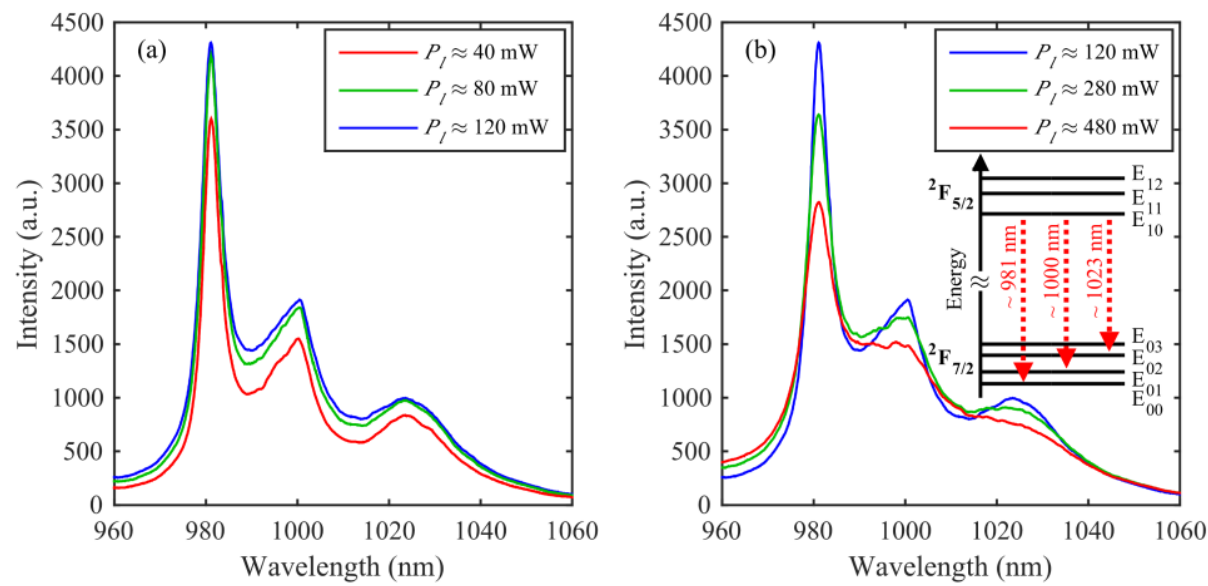

Figure 5.10 Spontaneous emission spectra of $\mathrm{KGd}_{0.43} \mathrm{Yb}_{0.57}\left(\mathrm{WO}_{4}\right)_{2}$ recorded with different launched pump power, $P_{l}$. (a) shows the intensification of emission with the increase of $P_{l}$ up to $120 \mathrm{~mW}$, whereas (b) shows the declination of detected emission for $P_{l}$ beyond $120 \mathrm{~mW}$. The spectra have been corrected for spectral response of the detection system. Inset of (b) illustrates the relevant transitions contributing to a large part of the observed emission at the corresponding wavelength.
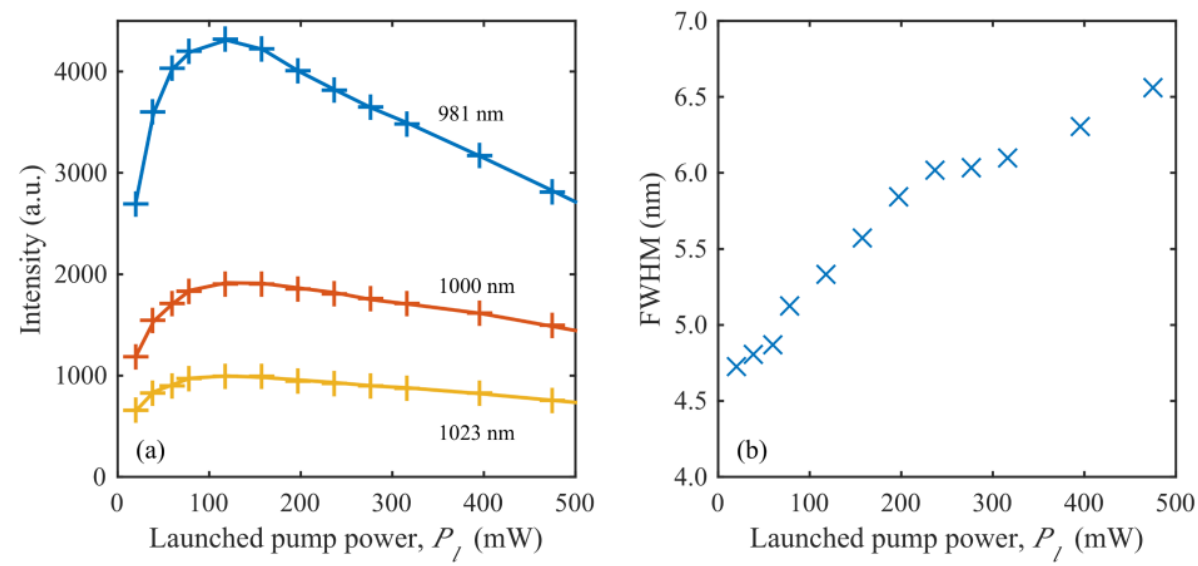

Figure 5.11 The evolution of (a) peak intensity of the major emission peaks and (b) linewidth (FWHM) of the decomposed peak at $\sim 981 \mathrm{~nm}$ with the increase of pump power. The lines are guides for the eye.

The results shown in Figure 5.10 and Figure 5.11 can be explained by considering the pump absorption, thermal effects, and gain results reported in previous subsections. Firstly, the maximum net gain determined from the sample is only $\sim 2.6 \mathrm{~dB}$, hence, the observed amplified spontaneous emission spectra are close to the effective emission cross-section spectrum. Secondly, the linewidth broadening of the emission peak near $981 \mathrm{~nm}$ wavelength signifies that thermal effect is present in the medium even under low $P_{l}$. The presence of thermal effect can be further confirmed by comparing the emission spectra in Figure 5.10(b) measured at $P_{l} \approx 120 \mathrm{~mW}$ (blue curve) and $P_{l} \approx 280 \mathrm{~mW}$ (green curve). The spectrum measured at $P_{l} \approx 280 \mathrm{~mW}$ exhibits lower 
intensity at the peaks near $981 \mathrm{~nm}$ and $1000 \mathrm{~nm}$ wavelengths but higher intensity at the dips near $990 \mathrm{~nm}$ and $1015 \mathrm{~nm}$. This is in line with the temperature-dependent behavior of the emission cross-section spectra shown in Figure 4.12, in which the increase of intensity at the dips can be explained by the contribution from the neighboring peaks due to linewidth broadening at higher temperature. Finally, the roll-off of the intensity in Figure 5.11(a) for $P_{l}>120 \mathrm{~mW}$ can be attributed to the reduction of transition crosssections due to pump-induced heating effect.

The visible luminescence spectra of $\mathrm{KGd}_{0.43} \mathrm{Yb}_{0.57}\left(\mathrm{WO}_{4}\right)_{2}$ were measured right after the NIR luminescence spectra under similar experiment condition. Luminescence spectra scanned with $0.5 \mathrm{~nm}$ step size under various $P_{l}$ values are shown in Figure 5.12. A reference background measurement was performed without the sample to identify the recorded emission attributed to the Ti:Sapphire pump source. The reference spectrum (black curve in Figure 5.12) shows that detected luminescence with wavelength $>590 \mathrm{~nm}$ is mainly contributed by the Ti:Sapphire pump source. Besides, several spikes, which are also apparent on other spectra, are observed in the reference spectrum. These spikes are possibly caused by the artifacts from the dispersive grating or by the detection of leaked pump when the grating turret was rotated to certain angles.

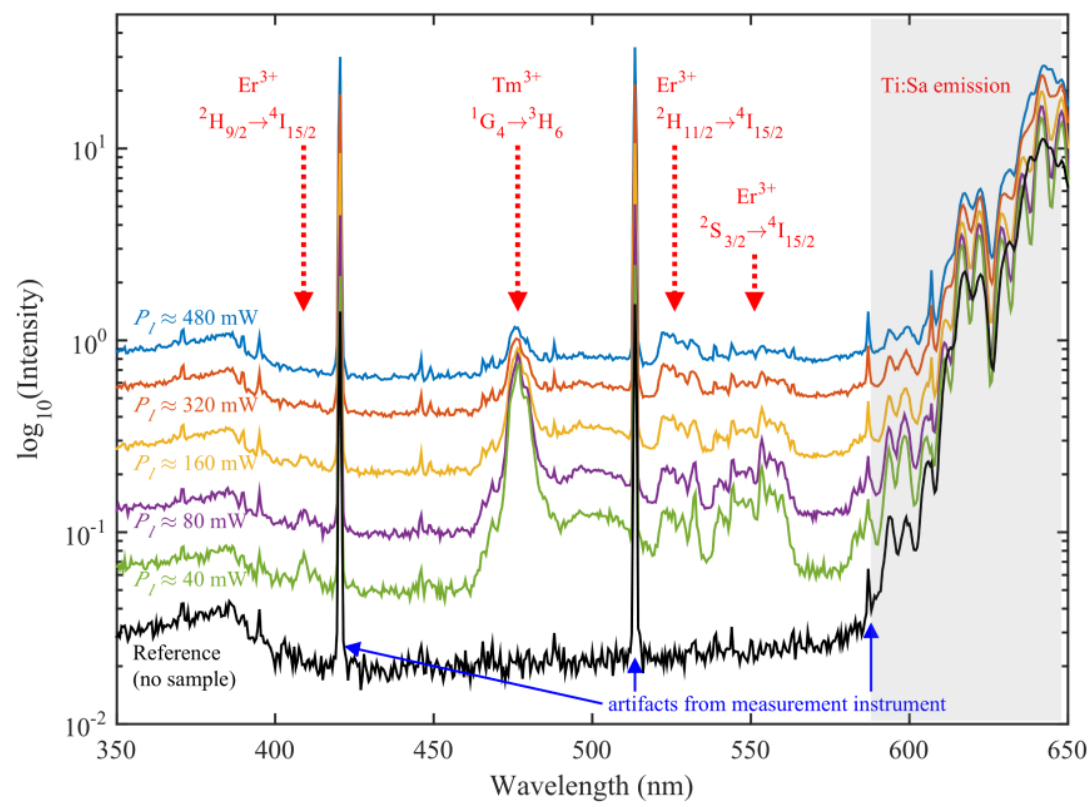

Figure 5.12 Luminescence spectra of $\mathrm{KGd}_{0.43} \mathrm{Yb}_{0.57}\left(\mathrm{WO}_{4}\right)_{2}$ within visible wavelength range in semi-log plot. The black curve shows the reference emission curve attributed to the pump source, which was collected after removing the sample from the beam path. The arrows indicate several major peaks persist in all measured curves, which are attributed to the artifacts from the measurement instrument. 
Based on the spectrum measured at $P_{l} \approx 40 \mathrm{~mW}$ (green curve), emission from $\mathrm{Tm}^{3+}$ due to ${ }^{1} \mathrm{G}_{4} \rightarrow{ }^{3} \mathrm{H}_{6}$ transition [161] is identified at $\sim 476.5 \mathrm{~nm}$. Besides, weaker emissions ascribed to $\mathrm{Er}^{3+}[162]$ at $\sim 409 \mathrm{~nm}\left({ }^{2} \mathrm{H}_{9 / 2} \rightarrow{ }^{4} \mathrm{I}_{15 / 2}\right), \sim 526 \mathrm{~nm}\left({ }^{2} \mathrm{H}_{11 / 2} \rightarrow{ }^{4} \mathrm{I}_{15 / 2}\right)$, and $\sim 552 \mathrm{~nm}\left({ }^{2} \mathrm{~S}_{3 / 2} \rightarrow{ }^{4} \mathrm{I}_{15 / 2}\right)$ are also apparent. The spectroscopic feature within 490-519 nm wavelength range could be due to cooperative up-conversion of $\mathrm{Yb}^{3+}$ ion pairs $[78,163]$ as the corresponding energy matches twice of the photons emitted in NIR region.

The emission spectra near $476.5 \mathrm{~nm}$ wavelength scanned with finer step size of $0.1 \mathrm{~nm}$ are shown in Figure 5.13(a) and the extracted maximum intensity values of the respective spectra are plotted in Figure 5.13(b). The maximum intensity increases rapidly with $P_{l}$ up to $P_{l} \approx 60 \mathrm{~mW}$, beyond which it decreases with further increase of $P_{l}$. The development of the visible spectra with increasing $P_{l}$ is very similar to that of the NIR spectra.

The results in Figure 5.12 and Figure 5.13 show that the response of the parasitic up-conversion luminescence to the increase of $P_{l}$ is similar to that of the NIR luminescence. Such parasitic luminescence could be related to the observed ETU process. Nevertheless, further investigation of energy transfer between the $\mathrm{Yb}^{3+}$ ions and the impurity ions would require preparation of new samples with controlled amount of impurity and in-depth spectroscopic investigations, which are beyond the scope of this thesis.
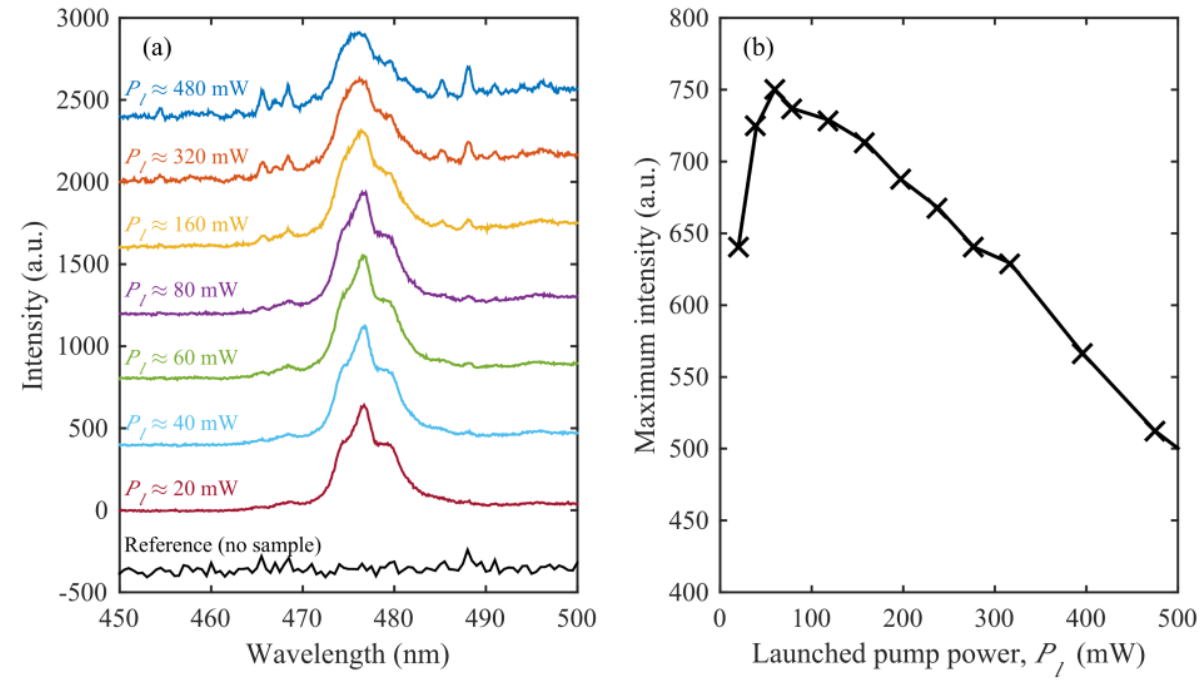

Figure 5.13 (a) Luminescence spectra near $476.5 \mathrm{~nm}$ wavelength from $\mathrm{KGd}_{0.43} \mathrm{Yb}_{0.57}\left(\mathrm{WO}_{4}\right)_{2}$ recorded at different launched pump power, $P_{l}$ settings. The baselines of the curves are shifted for better clarity. The black curve at the bottom shows the reference emission curve attributed to the pump source, which was collected after removing the sample from the beam path. (b) Change of maximum intensity of the emission peak near $476.5 \mathrm{~nm}$ wavelength with $\boldsymbol{P}_{l}$. The values are calculated from the curves which were normalized to the respective intensity at $450 \mathrm{~nm}$ wavelength. The line in (b) serves as guide for the eye. 


\subsection{Discussions}

\section{Gain limiting factors}

The limitation of the material gain can be evaluated using theoretical gain cross-section. Consider the gain coefficient defined by Eq. 2.26 which is shown as follows

$$
g=\sigma_{e m}\left(\lambda_{s}, T\right) N_{1}-\sigma_{a b s}\left(\lambda_{s}, T\right) N_{0} .
$$

The gain cross-section $\sigma_{g}$ can be deduced using an inversion parameter $\beta=N_{l} / N_{Y b}$ [22],

$$
\sigma_{g}=\beta \sigma_{e m}\left(\lambda_{s}, T\right)-(1-\beta) \sigma_{a b s}\left(\lambda_{s}, T\right) \text {. }
$$

With a pump wavelength at $932 \mathrm{~nm}$, the temperature-dependent maximum inversion $\beta_{\max }(T)$ can be determined at transparency for the pump photon [20]

$$
\beta_{\max }(T)=f_{00}(T) /\left[f_{00}(T)+f_{12}(T)\right],
$$

where $f_{00}(T)$ and $f_{12}(T)$ represent the respective fractional population at Stark level with energy $E_{00}$ and $E_{12}$ which can be calculated with Eqs. 2.10 and 2.11.

The theoretical $\sigma_{g}$ at $981 \mathrm{~nm}$ wavelength is calculated using cross-section data shown in Figure 5.4(a) and level energies of $\mathrm{KY}\left(\mathrm{WO}_{4}\right)_{2}: \mathrm{Yb}^{3+}$ [71]. The solid line in Figure 5.14 represents the calculated $\sigma_{g}$ at maximum inversion $\beta_{\max }$. The dash lines are $\sigma_{g}$ obtained with lower population inversion which could be caused by insufficient pump intensity, quenched ions, incomplete overlap of pump and signal beams etc....

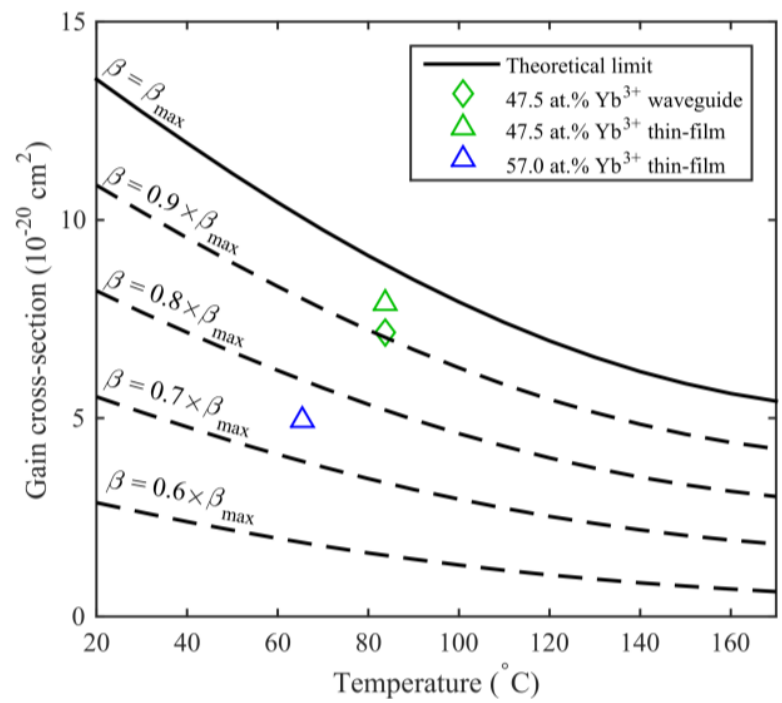

Figure 5.14 Influence of temperature $T$ and inversion condition $\beta$ on the gain crosssection. The lines are calculated values and the symbols are experimentally achieved gain cross-section. The gain data for amplifier with 47.5 at.\% $\mathrm{Yb}^{3+}$ is taken from [20]. 
The symbols in Figure 5.14 represents the experimentally achieved $\sigma_{g}$ values in $\mathrm{KRE}\left(\mathrm{WO}_{4}\right)_{2}$ waveguide and epitaxial layers, calculated by dividing the reported gain coefficient by the $\mathrm{Yb}^{3+}$ concentration. The effective $\sigma_{g}$ achieved in $\mathrm{KGd}_{0.43} \mathrm{Yb}_{0.57}\left(\mathrm{WO}_{4}\right)_{2}$ is $4.95 \times 10^{-20} \mathrm{~cm}^{2}$. Considering the calculated temperature of $\sim 65.5{ }^{\circ} \mathrm{C}$ in the sample, the average inversion is $\sim 75 \%$ of $\beta_{\max }\left(65.5^{\circ} \mathrm{C}\right)$ throughout the layer, which corresponds to $\beta=0.75 \times 0.874=0.65$. The performance of the amplifiers can be improved by (i) enhancing the population inversion, and (ii) efficient extraction of heat from the pumped region. The overall inversion is limited by quenched ions and ETU process. Assuming that the average temperature in the pumped volume is maintained at $30{ }^{\circ} \mathrm{C}$ by applying active cooling and $\beta$ remains unchanged, the $\sigma_{g}$ can be increased to $6.43 \times 10^{-}$ ${ }^{20} \mathrm{~cm}^{2}$, which is equivalent to $\sim 30 \%$ of gain enhancement.

The gain results for $\mathrm{KGd}_{0.447} \mathrm{Lu}_{0.078} \mathrm{Yb}_{0.475}\left(\mathrm{WO}_{4}\right)_{2}$ [20], which corresponds to 47.5 at. $\% \mathrm{Yb}^{3+}$, are also included in Figure 5.14 for comparison purpose. The results in [20] was based on transition cross-sections derived from weighted-average crosssection data of the constituent hosts. The peak $\sigma_{a b s}$ at $\sim 981 \mathrm{~nm}$ deduced in [20] $\left(9.45 \times 10^{-20} \mathrm{~cm}^{2}\right)$ is considerably lower than the $\sigma_{a b s}$ measured in this work at $20{ }^{\circ} \mathrm{C}$ $\left(\sim 13 \times 10^{-20} \mathrm{~cm}^{2}\right)$ but equivalent to the $\sigma_{a b s}$ data deduced in this work at $\sim 84{ }^{\circ} \mathrm{C}$. The $\sigma_{g}$ values of $\mathrm{KGd}_{0.447} \mathrm{Lu}_{0.078} \mathrm{Yb}_{0.475}\left(\mathrm{WO}_{4}\right)_{2}$ are plotted in Figure 5.14 with the assumption that the pump-induced heating effects are applicable to [20] and the effective temperature in the device was $84{ }^{\circ} \mathrm{C}$. The net gain reported for $\mathrm{KGd}_{0.447} \mathrm{Lu}_{0.078} \mathrm{Yb}_{0.475}\left(\mathrm{WO}_{4}\right)_{2}$ waveguide amplifier was $935 \mathrm{~dB} / \mathrm{cm}$ (green diamond symbol). The corresponding $\sigma_{g}$ is $7.18 \times 10^{-20} \mathrm{~cm}^{2}$ the maximum $\beta$ achievable in waveguide configuration is limited by the pump/signal overlapping factor of $88 \%$ [20]. The gain obtained by launching optical beams perpendicular to the $25 \mu \mathrm{m}$ $\mathrm{KGd}_{0.447} \mathrm{Lu}_{0.078} \mathrm{Yb}_{0.475}\left(\mathrm{WO}_{4}\right)_{2}$ thin-film was $1028 \mathrm{~dB} / \mathrm{cm}$, hence $\sigma_{g}$ is $7.89 \times 10^{-20} \mathrm{~cm}^{2}$. The inversion conditions for $\mathrm{KGd}_{0.447} \mathrm{Lu}_{0.078} \mathrm{Yb}_{0.475}\left(\mathrm{WO}_{4}\right)_{2}$ waveguide and thin-film already approach the upper limit. Hence, a practical way of further improving the device is via device cooling. By cooling the waveguide amplifier to $30{ }^{\circ} \mathrm{C}$, an increase of $\sigma_{g}$ up to $42 \%\left(10.20 \times 10^{-20} \mathrm{~cm}^{2}\right)$ is anticipated.

\section{Extra decay process reported in the literature}

The numerical results presented in this chapter show that ETU process increases the absorbed pump power, temperature, and threshold pump power for the signal to reach transparency. The rate of change of the density of excited ions due to ETU process is proportional to the square of density of excited ions. The reduced population inversion caused by ETU leads to reduction of signal gain.

The ETU process detected in this work coincides with experimental observations from heavily $\mathrm{Yb}^{3+}$-doped thin-disc lasers. Many groups had tried to employ high $\mathrm{Yb}^{3+}$ concentration YAG, $\mathrm{LuAG}$, or $\mathrm{Lu}_{2} \mathrm{O}_{3}$ thin layer for thin-disc lasers in order to achieve 
high pump absorption within the layer. However, abnormalities are observed for layers with $\mathrm{Yb}^{3+}$ concentration $>15$ at.\%. Extra decay process in $\mathrm{YAG}: \mathrm{Yb}^{3+}$ has been reported by Larionov et al. in a conference proceeding [151]. The rate of the extra decay is found to be comparable with the rate of decay due to spontaneous emission and it exhibits non-linear dependence to the excited $\mathrm{Yb}^{3+}$ ions. Besides, heat generation beyond the fractional heat load due to quantum defect has been observed and the additional heat generated is associated with the mentioned extra decay process. Separate research group has also reported the presence of nonlinear loss mechanism based on laser experiments using YAG: $\mathrm{Yb}^{3+}$ ceramic [164] and LuAG: $\mathrm{Yb}^{3+}$ crystal [165]. In both reports, laser experiments were repeated on high $\mathrm{Yb}^{3+}$ concentration samples using output couplers with different transmissivity. The use of output coupler with higher transmissivity effectively increases the excitation density needed to achieve laser action. It is reported that the laser output power decreases rapidly after certain pump power value for both gain materials when output coupler with high transmissivity is used [164, 165]. The drastic change of laser power cannot be explained by the temperature dependence of the transition cross-sections alone and it is attributed to additional nonlinear decay mechanism which takes place at high density of excited ions. Based on photoconductivity measurement on $\mathrm{YAG}: \mathrm{Yb}^{3+}$ and $\mathrm{Lu}_{2} \mathrm{O}_{3}: \mathrm{Yb}^{3+}$, Brandt et al. suggested that cooperative up-conversion involving 2-3 exited $\mathrm{Yb}^{3+}$ ions might be responsible to the observed unknown loss in heavily doped gain media [166]. Nevertheless, there is a lack of further explanation on the direct correlation between the photoconductivity measurement and the nonlinear loss identified from the laser experiment by the same research group [167].

Based on the abovementioned reports, the ETU process identified in this work could be relevant for other high $\mathrm{Yb}^{3+}$ concentration media as well. Even though the nature of the ETU process and quenched ions has not been fully understood, the experimental approaches, particularly the lifetime measurements using confocal setup, as well as the theoretical gain model which takes into account the thermal effects, ETU process, and quenched ions could be useful for other high $\mathrm{Yb}^{3+}$ concentration gain media. 


\subsection{Summary}

The pump absorption, signal gain, and luminescence spectra in $32 \mu \mathrm{m}$ thick $\mathrm{KGd}_{0.43} \mathrm{Yb}_{0.57}\left(\mathrm{WO}_{4}\right)_{2}$ epitaxial layer have been investigated experimentally and numerically. A theoretical gain model taking into account the pump-induced thermal effects has been developed and applied to study the pump absorption and signal gain characteristics of the sample.

The experimental pump absorption results reveal nonsaturable pump absorption as compared to the theoretical prediction, which can be adequately explained by assuming $17 \%$ of quenched ions in addition to the ETU process. Both factors lead to higher temperature in the pumped volume, higher threshold pump power required for the signal to achieve transparency, and lower signal net gain. The average temperature values in the pumped volume estimated at the highest launched pump power values employed in the experiment is $\sim 65.5^{\circ} \mathrm{C}$.

Taking into account the pump-induced heating effects, the net gain value determined from the signal enhancement data acquired with pump-probe optical experiment is $2.616 \mathrm{~dB}$ at $981 \mathrm{~nm}$ signal wavelength. The high net gain per unit length figure of $817 \mathrm{~dB} / \mathrm{cm}$ achieved in the samples is encouraging as no special thermal management was applied to the sample during the gain measurement. It is anticipated that efficient heat removal and cooling measures which maintain the sample's temperature at $30{ }^{\circ} \mathrm{C}$ may lead to $\sim 30 \%$ improved gain performance.

The luminescent spectra collected under high pump excitation condition show broadened emission peaks with the increase of launched pump power, hence justifying the finding that significant heat is generated within the sample. Besides, traces of impurities such as $\mathrm{Er}^{3+}$ and $\mathrm{Tm}^{3+}$ have been found. Cooperative luminescence at $\sim 500 \mathrm{~nm}$ due to the interaction of two excited $\mathrm{Yb}^{3+}$ ions could also occur. Nevertheless, the influence of these parasitic upconversion luminescence on the gain is not clear at the moment. Further experimental work is needed to determine the origin of ETU process and quenched ions as well as to quantify the impact of parasitic upconversion processes on the signal gain. 


\section{Chapter 6}

\section{Conclusions}

This thesis explains the research work concerning high $\mathrm{Yb}^{3+}$ concentration $\mathrm{KRE}\left(\mathrm{WO}_{4}\right)_{2}$ epitaxial layers, where RE stands for $\mathrm{Gd}, \mathrm{Lu}, \mathrm{Y}$, or the combination of these elements, which are catered for optical amplification purpose. Prior to this work, $\mathrm{KRE}\left(\mathrm{WO}_{4}\right)_{2}$ high-gain waveguide amplifiers based on epitaxial layer with 47.5 at.\% $\mathrm{Yb}^{3+}$ had been achieved. In-depth investigations on the optical properties of the epitaxial layers with $>50$ at. $\% \mathrm{Yb}^{3+}$ have been conducted in this work via experimental and numerical means to evaluate the potential of these layers for the realization of waveguide amplifiers with superior net gain per unit length performance.

High $\mathrm{Yb}^{3+}$ concentration $\operatorname{KRE}\left(\mathrm{WO}_{4}\right)_{2}$ layers have been grown on undoped $\mathrm{KY}\left(\mathrm{WO}_{4}\right)_{2}$ substrate by Dr. Shanmugam Aravazhi using liquid phase epitaxy technique. A lattice engineering approach has been adopted for the growth of the active epitaxial layers to simultaneously meet the requirements of a) maintaining lattice matching condition, b) increasing index of refraction, and c) maximizing the concentration of $\mathrm{Yb}^{3+}$. The composition of the sample with highest $\mathrm{Yb}^{3+}$ concentration has been characterized as $\mathrm{KGd}_{0.43} \mathrm{Yb}_{0.57}\left(\mathrm{WO}_{4}\right)_{2}$ and the refractive index contrast between the layer and the substrate is found to be $\sim 0.0123$ at $981 \mathrm{~nm}$ wavelength. Polished $\mathrm{KGd}_{0.43} \mathrm{Yb}_{0.57}\left(\mathrm{WO}_{4}\right)_{2}$ sample with layer thickness of $\sim 32 \mu \mathrm{m}$ has been prepared for spectroscopic and gain measurements. Besides, $\mathrm{KLu}\left(\mathrm{WO}_{4}\right)_{2}: \mathrm{Yb}^{3+}$ epitaxial layer grown on undoped $\mathrm{KLu}\left(\mathrm{WO}_{4}\right)_{2}$ substrate using top-seeded solution growth technique has been provided by Dr. Joan J. Carvajal from Universitat Rovira i Virgili, Tarragona. Due to the closely matched lattice parameters between $\mathrm{KLu}\left(\mathrm{WO}_{4}\right)_{2}$ and $\mathrm{KYb}\left(\mathrm{WO}_{4}\right)_{2}$, thick epitaxial layer (i.e. $>100 \mu \mathrm{m}$ ) with substantially higher $\mathrm{Yb}^{3+}$ concentration can be grown successfully. The composition of the layer has been characterized as $\mathrm{KLu}_{0.24} \mathrm{Yb}_{0.76}\left(\mathrm{WO}_{4}\right)_{2}$, which means that it contains 76 at.\% $\mathrm{Yb}^{3+}$. The polished layer thickness of the $\mathrm{KLu}_{0.24} \mathrm{Yb}_{0.76}\left(\mathrm{WO}_{4}\right)_{2}$ is $\sim 124 \mu \mathrm{m}$.

As the luminescent lifetime of high $\mathrm{Yb}^{3+}$ concentration material is typically hampered by radiation trapping effect which elongates the measured lifetime, a novel confocal lifetime measurement setup has been devised and tested. The setup is based on the concept of confocal volume detection which requires i) a pump volume which is as small as possible, and ii) effective discrimination of luminescence originated out of the non-confocal region from the detection system. As compared to other existing approaches being used to mitigate radiation trapping effect, such as measuring on 
diluted powdered sample or measuring with pinholes followed by an extrapolation procedure, the proposed setup allows direct measurement of lifetime in a nondestructive manner. The setup has been used to determine the lifetime on $\mathrm{KRE}\left(\mathrm{WO}_{4}\right)_{2}: \mathrm{Yb}^{3+}$ epitaxial layers with $\mathrm{Yb}^{3+}$ ranging from 1.2 at.\% to 76 at.\%. The measured lifetime results fall within the range of reported lifetime values measured using other approaches. Besides, effective suppression of radiation trapping effect using the proposed setup has been demonstrated. The lifetime obtained from sample with 1.2 at. $\%$ is $245 \pm 3 \mu \mathrm{s}$, whereas the lifetime values for $\mathrm{KGd}_{0.43} \mathrm{Yb}_{0.57}\left(\mathrm{WO}_{4}\right)_{2}$ and $\mathrm{KLu}_{0.24} \mathrm{Yb}_{0.76}\left(\mathrm{WO}_{4}\right)_{2}$ are $228 \pm 10 \mu$ s and $222 \pm 9 \mu \mathrm{s}$, respectively. Hence, concentration dependent lifetime quenching is rather weak even though these layers exhibit high amount of $\mathrm{Yb}^{3+}$ ions. Apart from that, power dependence of the luminescence decay has been studied. It is found that the time-resolved luminescence curves exhibit nonexponential decay, which indicates the presence of energy transfer upconversion (ETU) process.

Absorption measurements have been performed on $\mathrm{KGd}_{0.43} \mathrm{Yb}_{0.57}\left(\mathrm{WO}_{4}\right)_{2}$ and $\mathrm{KLu}_{0.24} \mathrm{Yb}_{0.76}\left(\mathrm{WO}_{4}\right)_{2}$ to determine the transition cross-sections in these samples. The impact of polarization disorientation on the measured peak absorption has been quantified. Besides, special care has been taken to reduce stray light in order to resolve the absorption peak of $\mathrm{KLu}_{0.24} \mathrm{Yb}_{0.76}\left(\mathrm{WO}_{4}\right)_{2}$ with high absorbance. The cross-sections determined from $\mathrm{KGd}_{0.43} \mathrm{Yb}_{0.57}\left(\mathrm{WO}_{4}\right)_{2}$ and $\mathrm{KLu}_{0.24} \mathrm{Yb}_{0.76}\left(\mathrm{WO}_{4}\right)_{2}$ are found to be similar to those of reported bulk $\mathrm{KRE}\left(\mathrm{WO}_{4}\right)_{2}: \mathrm{Yb}^{3+}$ crystals. The maximum absorption crosssection and emission cross-sections in these films are about $1.3 \times 10^{-19} \mathrm{~cm}^{2}$ and $1.6 \times 10^{-19} \mathrm{~cm}^{2}$, respectively. The difference of cross-section spectra obtained from $\mathrm{KGd}_{0.43} \mathrm{Yb}_{0.57}\left(\mathrm{WO}_{4}\right)_{2}$ and $\mathrm{KLu}_{0.24} \mathrm{Yb}_{0.76}\left(\mathrm{WO}_{4}\right)_{2}$ is marginal even though the $\mathrm{Yb}^{3+}$ concentration in these layers differs by 19 at.\%.

Temperature-dependent measurements have been performed on $\mathrm{KGd}_{0.43} \mathrm{Yb}_{0.57}\left(\mathrm{WO}_{4}\right)_{2}$ to quantify the change of spectroscopic parameters with temperature. This is because intensely pumped $\mathrm{Yb}^{3+}$-doped devices often operate at elevated temperature primarily due to the conversion of energy difference between the pump and luminescence photons into heat. It has been confirmed that the lifetime has no significant temperature dependence. On the other hand, a strong dependence of the absorption cross-section, especially on the major absorption peaks at $\sim 932 \mathrm{~nm}$ and $\sim 981 \mathrm{~nm}$, on sample's temperature has been detected. By increasing the temperature on the sample from $20{ }^{\circ} \mathrm{C}$ to $170{ }^{\circ} \mathrm{C}$, a reduction of cross-section by $\sim 40 \%$ and $\sim 52 \%$ has been determined for the absorption peak at $\sim 932 \mathrm{~nm}$ and $\sim 981 \mathrm{~nm}$, respectively. With the aid of a simple model, the reduction of peak absorption cross-section with increasing temperature can be explained by two effects, the reduced fractional population of the relevant Stark level and the linewidth broadening. When the temperature is increased from $20^{\circ} \mathrm{C}$ to $170{ }^{\circ} \mathrm{C}$, the fractional population of the relevant 
Stark level is reduced by $\sim 18 \%$. The linewidths of the decomposed absorption peaks near $932 \mathrm{~nm}$ and $981 \mathrm{~nm}$ is $\sim 1.37$ and $\sim 1.72$ times broader at $170{ }^{\circ} \mathrm{C}$ than at $20{ }^{\circ} \mathrm{C}$, respectively.

Owing to the strong dependency of transition cross-sections to device's temperature, a numerical gain model taking into account the pump-induced thermal effects has been established. In addition, pump-probe measurements have been conducted to examine the pump absorption and signal gain in $\mathrm{KGd}_{0.43} \mathrm{Yb}_{0.57}\left(\mathrm{WO}_{4}\right)_{2}$ epitaxy layer. The comparison of the experimental and numerical pump absorption results reveals non-saturation pump absorption behavior. Such effect has been reported in other high active ion concentration materials and it is attributed to separate class of ions which is rapidly quenched from the excited state. By assuming $17 \%$ of $\mathrm{Yb}^{3+}$ ions are quenched in addition to ETU process, the calculated result from the numerical model match reasonably to the experimental data. Apart from the pump absorption, the temperature profiles of the sample have been modeled as well. The results show that at the highest launched pump power applied in the gain experiment, the average temperature within the pumped volume is $\sim 65.5^{\circ} \mathrm{C}$. The maximum net gain achieved in $\mathrm{KGd}_{0.43} \mathrm{Yb}_{0.57}\left(\mathrm{WO}_{4}\right)_{2}$ is calculated as $2.62 \mathrm{~dB}$, or $817 \mathrm{~dB} / \mathrm{cm}$. Two gain limiting factors, which are the population inversion and the temperature of the device, have been identified by comparing the effective gain cross-sections achieved in the sample to theoretical gain cross-section versus temperature curves. Assuming that the temperature of the sample can be reduced to $30{ }^{\circ} \mathrm{C}$ via active cooling, the net gain per unit lengths could be enhanced by $\sim 30 \%$.

In overall, it has been demonstrated experimentally that $\mathrm{KRE}\left(\mathrm{WO}_{4}\right)_{2}: \mathrm{Yb}^{3+}$ epitaxial layers with $\mathrm{Yb}^{3+}>50 \%$ exhibit spectroscopic properties which are very similar to those of bulk $\mathrm{KRE}\left(\mathrm{WO}_{4}\right)_{2}: \mathrm{Yb}^{3+}$ crystals. This indicates that these $\mathrm{KRE}\left(\mathrm{WO}_{4}\right)_{2}: \mathrm{Yb}^{3+}$ layers can be utilized for amplifiers and lasers. Nevertheless, the ETU process, quenched ions, and thermal effects would affect the gain achieved in these layers. Using pump wavelength of $932 \mathrm{~nm}$ and signal wavelength of $981 \mathrm{~nm}$, a rather high net gain per unit length of $817 \mathrm{~dB} / \mathrm{cm}$ is determined despite sub-optimal thermal and inversion conditions. The gain value demonstrated represents only a fraction of the theoretical gain of the material. Efficient heat dissipation from the sample is expected to improve the gain substantially. 


\section{Outlook}

In Chapter 5, it has been shown that the experimental net gain values obtained are limited by population inversion condition and thermal issue. While the former factor is well known for rare-earth-ion-doped devices, the later factor has received considerably less attention in the field of rare-earth-ion-doped waveguide amplifier. Based on the findings in this thesis, further work can be done in the following aspects to fully exploit the gain in high $\mathrm{Yb}^{3+}$ concentration $\mathrm{KRE}\left(\mathrm{WO}_{4}\right)_{2}$ epitaxial layers:

\section{Efficient heat extraction to external environment}

Thermal management on the $\mathrm{KRE}\left(\mathrm{WO}_{4}\right)_{2}: \mathrm{Yb}^{3+}$ amplifiers provides a straightforward approach to enhance the net gain. A potential heat dissipation solution for the $\mathrm{KRE}\left(\mathrm{WO}_{4}\right)_{2}$ waveguide amplifiers is depicted in Figure 6.1. A heat spreader placed on top of amplifier is suggested as the thickness of the top cladding layer is usually $<50 \mu \mathrm{m}$ whereas the substrate can be $>1 \mathrm{~mm}$ thick. A diamond heat spreader with thermal conductivity exceeding $100 \mathrm{Wm}^{-1} \mathrm{~K}^{-1}$ can be used. Alternatively, cost-effective sapphire window with thermal conductivity of $\sim 30 \mathrm{Wm}^{-1} \mathrm{~K}^{-1}$ may serve as heat spreader. The amplifier and the heat spreader can be attached to the metal block holder through direct soldering, thermal grease, or indium foil. Heat removal from the metal block can be done by attaching the metal block to a heat sink (passive cooling) or to a Peltier element (active cooling).

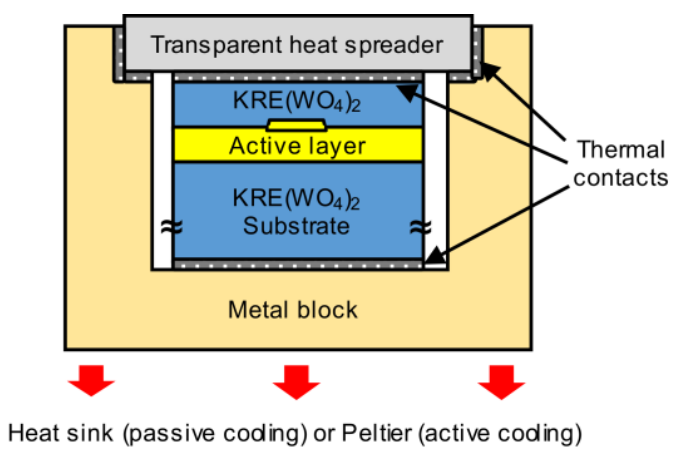

Figure 6.1 Schematic diagram of the proposed heat dissipation solution for $\mathrm{KRE}\left(\mathrm{WO}_{4}\right)_{2}$ waveguide amplifiers. The cross-sectional structure of the amplifier is represented by blue and yellow boxes. The active layer is patterned into ridge structure using lithography tool and an undoped $\mathrm{KRE}\left(\mathrm{WO}_{4}\right)_{2}$ cladding layer is grown on the patterned active layer. The thermal contacts can be realized using metal solder, thermal grease, or indium foil. The metal block can be attached onto a heat sink for passive cooling or a Peltier element for active cooling. 


\section{Flip-chip integrated waveguide amplifier}

One of the limitations of the perpendicular gain measurement on epitaxial layer is that smaller focused beam size could not be used as the beam would strongly diverge within the layer, hence the inversion condition and pump efficiency achieved are suboptimal. In principle, this can be overcome by employing waveguide structure with small waveguide mode size. However, integration of such waveguide with external optical interconnects, which is the eventual application for the waveguide amplifiers, would lead to poor mode overlapping which diminishes the overall net gain [57]. A flip-chip integrated waveguide amplifier would allow separation of pump coupling structure and gain structure within the integrated chip. The waveguide amplifier can be flip-chip bonded onto a $\mathrm{Si}_{3} \mathrm{~N}_{4}$ waveguide interposer. $\mathrm{Si}_{3} \mathrm{~N}_{4}$ waveguides exhibit low loss over a wavelength range of $400-2350 \mathrm{~nm}$ and the waveguides can be optimized for low loss coupling to single mode fibers $(<0.5 \mathrm{~dB})$ [168], hence high coupling efficiency can be achieved if pigtailed diode laser was used as the pump source. The transferred of optical beams between the $\mathrm{Si}_{3} \mathrm{~N}_{4}$ waveguides and $\mathrm{KRE}\left(\mathrm{WO}_{4}\right)_{2}: \mathrm{Yb}^{3+}$ can be achieved via adiabatic mode transfer [14]. This in turn permits the use of thin $\mathrm{KRE}\left(\mathrm{WO}_{4}\right)_{2}: \mathrm{Yb}^{3+}$ layer to realize high pump intensity within the amplifier because the thickness of the active layer does not affect the mode transfer mechanism significantly. 



\section{Appendix}

\section{Review on waveguide amplifiers}

The data used to plot Figure 1.2 is presented in Table A using the following notations:

$\lambda_{s}$ - signal wavelength

$\lambda_{p}$ - pump wavelength

$N_{d}$ - active ion concentration

$l$ - device length

$G$ - internal net gain

$G / l-$ internal net gain per unit length

$P$ - pump power used to achieve the reported $G$ or $G / l$

Table A Collection data for waveguide amplifiers reported with net gain.

\begin{tabular}{|c|c|c|c|c|c|c|c|c|}
\hline $\begin{array}{c}\lambda s \\
(\mathrm{~nm})\end{array}$ & $\begin{array}{c}\lambda_{p} \\
(\mathrm{~nm})\end{array}$ & Material & $N_{d}\left(\mathrm{~cm}^{-3}\right)$ & $l$ & $\begin{array}{c}G \\
(\mathrm{~dB})\end{array}$ & $\begin{array}{c}G / l \\
(\mathrm{~dB} / \mathrm{cm})\end{array}$ & $\underset{(\mathrm{mW})}{P}$ & $\begin{array}{l}\text { Year } \\
\& \text { Ref }\end{array}$ \\
\hline 1530 & 514.5 & $\mathrm{Er}^{3+}$ fiber & $1.8 \times 10^{18}$ & 13 meter & 22 & $0.0169^{*}$ & 100 & $\begin{array}{l}1989 \\
{[42]}\end{array}$ \\
\hline 1536 & 532 & $\mathrm{Er}^{3+}$ fiber & $0.1 \mathrm{wt} \%$ & 5 meter & 34 & 0.068 & 25 & $\begin{array}{l}1989 \\
{[43]}\end{array}$ \\
\hline 1535 & 980 & Silica: $\mathrm{Er}^{3+}$ & 0.55 wt. $\%$ & $19.4 \mathrm{~cm}$ & 13.7 & 0.65 & 640 & $\begin{array}{l}1992 \\
{[44]}\end{array}$ \\
\hline 1533 & 980 & $\begin{array}{l}\text { Phosphosilicate } \\
: \mathrm{Er}^{3+}\end{array}$ & $0.48 w \mathrm{t} \%$ & $7.5 \mathrm{~cm}$ & 5 & 0.67 & 420 & $\begin{array}{l}1993 \\
{[46]}\end{array}$ \\
\hline 1533 & 980 & Silica: $\mathrm{Er}^{3+}$ & 3800 ppm & $23 \mathrm{~cm}$ & 9.4 & $0.409^{*}$ & 99 & $\begin{array}{l}1993 \\
{[45]}\end{array}$ \\
\hline 1530 & 1480 & $\mathrm{Al}_{2} \mathrm{O}_{3}: \mathrm{Er}^{3+}$ & $2.7 \times 10^{20}$ & $4 \mathrm{~cm}$ & 2.3 & 0.575 & 9 & $\begin{array}{l}1996 \\
{[47]} \\
\end{array}$ \\
\hline 1535 & 980 & Phosphate: $\mathrm{Er}^{3+}$ & $5.3 \times 10^{20}$ & $1 \mathrm{~cm}$ & 4.1 & 4.1 & $\sim 21$ & $\begin{array}{l}1997 \\
{[48]}\end{array}$ \\
\hline 1550 & 980 & Silica: $\mathrm{Er}^{3+}$ & NA & $5 \mathrm{~cm}$ & $6.4^{*}$ & $1.28^{*}$ & 175 & $\begin{array}{l}2002 \\
{[169]}\end{array}$ \\
\hline 1530 & 1475 & $\mathrm{TeO}_{2}: \mathrm{Er}^{3+}$ & $\sim 2.2 \times 10^{20}$ & $5 \mathrm{~cm}$ & 14 & 2.8 & $\sim 200$ & $\begin{array}{l}2010 \\
{[49]}\end{array}$ \\
\hline 1532 & 976 & $\mathrm{Al}_{2} \mathrm{O}_{3}: \mathrm{Er}^{3+}$ & $1.92 \times 10^{20}$ & $12.9 \mathrm{~cm}$ & 20 & $1.55^{*}$ & 30 & $\begin{array}{l}2014 \\
{[37]} \\
\end{array}$ \\
\hline 1535 & 980 & $\mathrm{TeO}_{2}: \mathrm{Er}^{3+}$ & $\sim 2.2 \times 10^{20}$ & $5 \mathrm{~cm}$ & $>14$ & $>2.8$ & $\sim 180$ & $\begin{array}{l}2015 \\
{[50]}\end{array}$ \\
\hline 1530 & 980 & $\begin{array}{l}\mathrm{Er}_{0.4} \mathrm{Y}_{1.6} \mathrm{SiO}_{5} \\
\text { silicates }\end{array}$ & $\sim 3.2 \times 10^{21}$ & $500 \mu \mathrm{m}$ & $1.5^{*}$ & 30 & NA & $\begin{array}{l}2014 \\
{[51]}\end{array}$ \\
\hline
\end{tabular}


Appendix

\begin{tabular}{|c|c|c|c|c|c|c|c|c|}
\hline 1535 & 984.5 & $\mathrm{KRE}\left(\mathrm{WO}_{4}\right)_{2}$ & $3.8 \times 10^{20}$ & $750 \mu \mathrm{m}$ & $\sim 1$ & 13 & NA & 2016 \\
\hline 1534 & 980 & $\begin{array}{l}\text { Phosphate glass } \\
: \mathrm{Er}^{3+} \& \mathrm{Yb}^{3+}\end{array}$ & $\begin{array}{l}7.5 \times 10^{20} \mathrm{Er} \\
11 \times 10^{20} \mathrm{Yb}\end{array}$ & $0.3 \mathrm{~cm}$ & 4.1 & $13.67^{*}$ & 150 & $\begin{array}{l}2004 \\
{[52]}\end{array}$ \\
\hline 1534 & 975 & $\begin{array}{l}\text { Phosphate glass } \\
: \mathrm{Er}^{3+} \& \mathrm{Yb}^{3+}\end{array}$ & NA & $3.1 \mathrm{~cm}$ & 15 & 4.8 & 460 & $\begin{array}{l}2006 \\
{[41]}\end{array}$ \\
\hline - & - & $\begin{array}{l}\text { Tantalum } \\
\text { pentoxide: } \mathrm{Yb}^{3+}\end{array}$ & $\sim 6.2 \times 10^{20}$ & - & - & $\begin{array}{c}78 \\
\text { (theory) }\end{array}$ & - & $\begin{array}{l}2014 \\
{[59]}\end{array}$ \\
\hline 981 & 932 & $\mathrm{KRE}\left(\mathrm{WO}_{4}\right)_{2}: \mathrm{Yb}^{3+}$ & $3.0 \times 10^{21}$ & $180 \mu \mathrm{m}$ & 16.83 & 935 & 60 & $\begin{array}{l}2012 \\
{[20]}\end{array}$ \\
\hline 1064 & 800 & Polymer: $\mathrm{Nd}^{3+}$ & $1.03 \times 10^{20}$ & $5.6 \mathrm{~cm}$ & 8 & 1.43 & 29 & $\begin{array}{l}2009 \\
{[55]}\end{array}$ \\
\hline 880 & 802 & $\mathrm{Al}_{2} \mathrm{O}_{3}: \mathrm{Nd}^{3+}$ & $\begin{array}{c}0.65 \times 10^{20} \\
1.4 \times 10^{20}\end{array}$ & $\begin{array}{c}4.1 \mathrm{~cm} \\
1 \mathrm{~cm}\end{array}$ & $\begin{array}{l}3.0 \\
1.57\end{array}$ & $\begin{array}{l}1.37 \\
1.57\end{array}$ & 45 & $\begin{array}{l}2010 \\
{[22]}\end{array}$ \\
\hline 1064 & 802 & $\mathrm{Al}_{2} \mathrm{O}_{3}: \mathrm{Nd}^{3+}$ & $\begin{array}{l}1.13 \times 10^{20} \\
1.68 \times 10^{20}\end{array}$ & $\begin{array}{c}4.1 \mathrm{~cm} \\
1.3-1.5 \mathrm{~cm}\end{array}$ & $\begin{array}{l}14.4 \\
8.2^{*}\end{array}$ & $\begin{array}{c}3.51 \\
6.3\end{array}$ & 45 & $\begin{array}{l}2010 \\
{[22]}\end{array}$ \\
\hline 1330 & 802 & $\mathrm{Al}_{2} \mathrm{O}_{3}: \mathrm{Nd}^{3+}$ & $\begin{array}{l}1.13 \times 10^{20} \\
1.68 \times 10^{20}\end{array}$ & $\begin{array}{c}4.1 \mathrm{~cm} \\
1.3-1.5 \mathrm{~cm}\end{array}$ & $\begin{array}{l}5.1 \\
2.5^{*}\end{array}$ & $\begin{array}{l}1.24 \\
1.93\end{array}$ & 45 & $\begin{array}{l}2010 \\
{[22]}\end{array}$ \\
\hline 873 & 800 & Polymer: $\mathrm{Nd}^{3+}$ & $\begin{array}{l}0.6 \times 10^{20} \\
1.03 \times 10_{20} \\
\end{array}$ & $\begin{array}{l}1.9 \mathrm{~cm} \\
1 \mathrm{~cm}\end{array}$ & $\begin{array}{l}2.8 \\
2.0\end{array}$ & $\begin{array}{c}1.47 \\
2.0\end{array}$ & 26 & $\begin{array}{l}2010 \\
{[56]}\end{array}$ \\
\hline 1064 & 800 & Polymer: $\mathrm{Nd}^{3+}$ & $\begin{array}{l}1.03 \times 10_{20} \\
1.03 \times 10_{20}\end{array}$ & $\begin{array}{l}4.4 \mathrm{~cm} \\
1 \mathrm{~cm}\end{array}$ & $\begin{array}{c}8 \\
5.7\end{array}$ & $\begin{array}{l}1.8 \\
5.7\end{array}$ & 26 & $\begin{array}{l}2010 \\
{[56]}\end{array}$ \\
\hline 880 & 802 & $\mathrm{Al}_{2} \mathrm{O}_{3}: \mathrm{Nd}^{3+}$ & $0.5 \times 10^{20}$ & $6 \mathrm{~cm}$ & $\sim 3.5^{*}$ & 0.58 & 55 & $\begin{array}{l}{[57]} \\
2011\end{array}$ \\
\hline 1064 & 810 & $\begin{array}{l}\text { YAG ceramic } \\
: \mathrm{Nd}^{3+}\end{array}$ & 2 at. $\%$ & $7 \mathrm{~mm}$ & 18.4 & 26.3 & 21 & $\begin{array}{l}2013 \\
{[58]}\end{array}$ \\
\hline
\end{tabular}

* Estimated from the data available in the cited work 


\section{Bibliography}

[1] R. Dangel, J. Hofrichter, F. Horst, D. Jubin, A. La Porta, N. Meier, I. M. Soganci, J. Weiss, and B. J. Offrein, "Polymer waveguides for electro-optical integration in data centers and high-performance computers," Optics Express 23, 4736 (2015).

[2] P. Rosenberg, S. Mathai, W. V. Sorin, M. McLaren, J. Straznicky, G. Panotopoulos, D. Warren, T. Morris, and M. R. T. Tan, "Low cost, injection molded 120 Gbps optical backplane," Journal of Lightwave Technology 30, 590 (2012).

[3] S. Imai, K. Takaki, S. Kamiya, H. Shimizu, J. Yoshida, Y. Kawakita, T. Takagi, K. Hiraiwa, H. Shimizu, T. Suzuki, N. Iwai, T. Ishikawa, N. Tsukiji, and A. Kasukawa, "Recorded low power dissipation in highly reliable 1060-nm VCSELs for "green" optical interconnection," IEEE Journal of Selected Topics in Quantum Electronics 17, 1614 (2011).

[4] H. Li, P. Wolf, P. Moser, G. Larisch, A. Mutig, J. A. Lott, and D. H. Bimberg, "Impact of the quantum well gain-to-cavity etalon wavelength offset on the high temperature performance of high bit rate 980-nm VCSELs," IEEE Journal of Quantum Electronics 50, 613 (2014).

[5] P. Westbergh, B. Kögel, J. S. Gustavsson, E. Haglund, R. Safaisini, A. Joel, M. Geen, R. Lawrence, and A. Larsson, "High-speed $850 \mathrm{~nm}$ VCSELs with $28 \mathrm{GHz}$ modulation bandwidth operating error-free up to $44 \mathrm{Gbit} / \mathrm{s}$," Electronics Letters 48, 1145 (2012).

[6] F. E. Doany, C. L. Schow, C. W. Baks, D. M. Kuchta, P. Pepeljugoski, L. Schares, R. Budd, F. Libsch, R. Dangel, F. Horst, B. J. Offrein, and J. A. Kash, "160 Gb/s bidirectional polymer-waveguide board-level optical interconnects using CMOS-based transceivers," IEEE Transactions on Advanced Packaging 32, 345 (2009).

[7] K. Schmidtke, F. Flens, A. Worrall, R. Pitwon, F. Betschon, T. Lamprecht, and R. Krahenbuhl, "960 Gb/s optical backplane ecosystem using embedded polymer waveguides and demonstration in a $12 \mathrm{G}$ SAS storage array," Journal of Lightwave Technology 31, 3970 (2013).

[8] D. D'Agostino, G. Carnicella, C. Ciminelli, P. Thijs, P. J. Veldhoven, H. Ambrosius, and M. Smit, "Low-loss passive waveguides in a generic InP foundry process via local diffusion of zinc," Optics Express 23, 25143 (2015).

[9] H. Park, A. W. Fang, O. Cohen, R. Jones, M. J. Paniccia, and J. E. Bowers, "A hybrid AlGaInAs-silicon evanescent amplifier," IEEE Photonics Technology Letters 19, 230 (2007).

[10] L. Chang, M. H. P. Pfeiffer, N. Volet, M. Zervas, J. D. Peters, C. L. Manganelli, E. J. Stanton, Y. Li, T. J. Kippenberg, and J. E. Bowers, "Heterogeneous integration of lithium niobate and silicon nitride waveguides for wafer-scale photonic integrated circuits on silicon," Optics Letters 42, 803 (2017). 
[11] A. Beling and J. C. Campbell, "Heterogeneously integrated photodiodes on silicon," IEEE Journal of Quantum Electronics 51, 0600506 (2015).

[12] J. Pu, K. P. Lim, D. K. Ng, V. Krishnamurthy, C. W. Lee, K. Tang, A. Y. Seng Kay, T. H. Loh, and Q. Wang, "Heterogeneously integrated III-V laser on thin SOI with compact optical vertical interconnect access," Optics Letters 40, 1378 (2015).

[13] L. Agazzi, J. D. B. Bradley, M. Dijkstra, F. Ay, G. Roelkens, R. Baets, K. Wörhoff, and M. Pollnau, "Monolithic integration of erbium-doped amplifiers with silicon-on-insulator waveguides," Optics Express 18, 27703 (2010).

[14] I. M. Soganci, A. La Porta, and B. J. Offrein, "Flip-chip optical couplers with scalable I/O count for silicon photonics," Optics Express 21, 16075 (2013).

[15] S. Uhlig and M. Robertsson, "Limitations to and solutions for optical loss in optical backplanes," Journal of Lightwave Technology 24, 1710 (2006).

[16] J. D. B. Bradley, R. Stoffer, A. Bakker, L. Agazzi, F. Ay, K. Wörhoff, and M. Pollnau, "Integrated $\mathrm{Al}_{2} \mathrm{O}_{3}: \mathrm{Er}^{3+}$ zero-loss optical amplifier and power splitter with 40-nm bandwidth," IEEE Photonics Technology Letters 22, 278 (2010).

[17] K. Vu, K. Yan, Z. Jin, X. Gai, D. Y. Choi, S. Debbarma, B. Luther-Davies, and S. Madden, "Hybrid waveguide from $\mathrm{As}_{2} \mathrm{~S}_{3}$ and Er-doped $\mathrm{TeO}_{2}$ for lossless nonlinear optics," Optics Letters 38, 1766 (2013).

[18] S. M. García-Blanco, M. Pollnau, and S. I. Bozhevolnyi, "Loss compensation in long-range dielectric-loaded surface plasmon-polariton waveguides," Optics Express 19, 25298 (2011).

[19] D. R. Zimmerman and L. H. Spiekman, "Amplifiers for the masses: EDFA, EDWA, and SOA amplets for metro and access applications," Journal of Lightwave Technology 22, 63 (2004).

[20] D. Geskus, S. Aravazhi, S. M. García-Blanco, and M. Pollnau, "Giant optical gain in a rare-earth-ion-doped microstructure," Advanced Materials 24, OP19 (2012).

[21] J. Leuthold, M. Mayer, J. Eckner, G. Guekos, H. Melchior, and Ch. Zellweger, "Material gain of bulk $1.55 \mu \mathrm{m}$ InGaAsP/InP semiconductor optical amplifiers approximated by a polynomial model," Journal of Applied Physics 87, 618 (1999).

[22] J. Yang, K. van Dalfsen, K. Wörhoff, F. Ay, and M. Pollnau, "High-gain $\mathrm{Al}_{2} \mathrm{O}_{3}: \mathrm{Nd}^{3+}$ channel waveguide amplifiers at $880 \mathrm{~nm}, 1060 \mathrm{~nm}$, and $1330 \mathrm{~nm}$," Applied Physics B 101, 119 (2010).

[23] M. J. F. Digonnet, Rare-earth-doped fiber lasers and amplifiers, 2nd ed. (Marcel Dekker, 2001).

[24] J. B. Héroux, T. Kise, M. Funabashi, T. Aoki, C. L. Schow, A. V. Rylyakov, and S. Nakagawa, "Energy-efficient 1060 -nm optical link operating up to $28 \mathrm{~Gb} / \mathrm{s}$," Journal of Lightwave Technology 33, 733 (2015).

[25] S. Aozasa, H. Masuda, and M. Shimizu, "S-band thulium-doped fiber amplifier employing high thulium concentration doping technique," Journal of Lightwave Technology 24, 3842 (2006). 
[26] Z. Li, A. M. Heidt, J. M. O. Daniel, Y. Jung, S. U. Alam, and D. J. Richardson, "Thulium-doped fiber amplifier for optical communications at $2 \mu \mathrm{m}$," Optics Express 21, 9289 (2013).

[27] F. Auzel, G. Baldacchini, L. Laversenne, and G. Boulon, "Radiation trapping and self-quenching analysis in $\mathrm{Yb}^{3+}, \mathrm{Er}^{3+}$, and $\mathrm{Ho}^{3+}$ doped $\mathrm{Y}_{2} \mathrm{O}_{3}$," Optical Materials 24, 103 (2003).

[28] L. Agazzi, K. Wörhoff, and M. Pollnau, "Energy-transfer-upconversion models, their applicability and breakdown in the presence of spectroscopically distinct ion classes: A case study in amorphous $\mathrm{Al}_{2} \mathrm{O}_{3}: \mathrm{Er}^{3+}$," The Journal of Physical Chemistry C 117, 6759 (2013).

[29] T. Takemoto, F. Yuki, H. Yamashita, S. Tsuji, Y. Lee, K. Adachi, K. Shinoda, Y. Matsuoka, K. Kogo, S. Nishimura, M. Nido, M. Namiwaka, T. Kaneko, T. Sugimoto, and K. Kurata, "100-Gbps CMOS transceiver for multilane optical backplane system with a $1.3 \mathrm{~cm}^{2}$ footprint," Optics Express 19, B777 (2011).

[30] C. S. Wang, Y. C. Chang, and L. A. Coldren, "35 Gbit/s error-free operation of $980 \mathrm{~nm}$ DBR laser with integrated electroabsorption modulator," Electronics Letters 43, 1227 (2007).

[31] W. Hofmann, M. Muller, A. Nadtochiy, C. Meltzer, A. Mutig, G. Bohm, J. Rosskopf, D. Bimberg, M. C. Amann, and C. Chang-Hasnain, "22-Gb/s Long Wavelength VCSELs," Optics Express 17, 17547 (2009).

[32] W. Hofmann, M. Muller, P. Wolf, A. Mutig, T. Grundl, G. Bohm, D. Bimberg, and M. C. Amann, "40 Gbit/s modulation of $1550 \mathrm{~nm}$ VCSEL," Electronics Letters 47, 270 (2011).

[33] T. Suzuki, S. Imai, S. Kamiya, K. Hiraiwa, M. Funabashi, Y. Kawakita, H. Shimizu, T. Ishikawa, and A. Kasukawa, "Reliability study of $1060 \mathrm{~nm} 25 \mathrm{Gbps}$ VCSEL in terms of high speed modulation," Proceedings SPIE 8276, 827604 (2012).

[34] J. D. B. Bradley, M. Costa e Silva, M. Gay, L. Bramerie, A. Driessen, K. Wörhoff, J. C. Simon, and M. Pollnau, "170 Gbit/s transmission in an erbiumdoped waveguide amplifier on silicon," Optics Express 17, 22201 (2009).

[35] J. D. B. Bradley, L. Agazzi, D. Geskus, F. Ay, K. Wörhoff, and M. Pollnau, "Gain bandwidth of $80 \mathrm{~nm}$ and $2 \mathrm{~dB} / \mathrm{cm}$ peak gain in $\mathrm{Al}_{2} \mathrm{O}_{3}: \mathrm{Er}^{3+}$ optical amplifiers on silicon," Journal of the Optical Society of America B 27, 187 (2010).

[36] D. Geskus, S. Aravazhi, E. H. Bernhardi, L. Agazzi, S. M. García-Blanco, and M. Pollnau, "150 dB/cm gain over $55 \mathrm{~nm}$ wavelength range near $1 \mu \mathrm{m}$ in an $\mathrm{Yb}$ doped waveguide amplifier," in Conference on Lasers and Electro-Optics 2012, OSA Technical Digest (Optical Society of America, 2012), CM1A.6.

[37] S. A. Vázquez-Córdova, M. Dijkstra, E. H. Bernhardi, F. Ay, K. Wörhoff, J. L. Herek, S. M. García-Blanco, and M. Pollnau, "Erbium-doped spiral amplifiers with $20 \mathrm{~dB}$ of net gain on silicon," Optics Express 22, 25993 (2014).

[38] M. J. Connelly, Semiconductor Optical Amplifiers (Springer, 2002).

[39] N. K. Dutta and Q. Wang, Semiconductor Optical Amplifiers (World Scientific Publishing, 2006). 
[40] M. Tassaert, S. Keyvaninia, D. Van Thourhout, W. M. J. Green, Y. Vlasov, and G. Roelkens, "An optically pumped nanophotonic InP/InGaAlAs optical amplifier integrated on a SOI waveguide circuit," Optical and Quantum Electronics 44, 513 (2012).

[41] G. Della Valle, S. Taccheo, P. Laporta, G. Sorbello, E. Cianci, and V. Foglietti, "Compact high gain erbium-ytterbium doped waveguide amplifier fabricated by Ag-Na ion exchange," Electronics Letters 42, 632 (2006).

[42] E. Desurvire, J. R. Simpson, and P. C. Becker, "High-gain erbium-doped traveling-wave fiber amplifier," Optics Letters 12, 888 (1987).

[43] M. C. Farries, P. R. Morkel, R. I. Laming, T. A. Birks, D. N. Payne, and E. J. Tarbox, "Operation of erbium-doped fiber amplifiers and lasers pumped with frequency-doubled Nd:YAG lasers," Journal of Lightwave Technology 7, 1473 (1989).

[44] T. Kitagawa, K. Hattori, K. Shuto, M. Yasu, M. Kobayashi, and M. Horiguchi, "Amplification in erbium-doped silica-based planar lightwave circuits," Electronics Letters 28, 1818 (1992).

[45] K. Hattori, T. Kitagawa, M. Oguma, M. Wada, J. Temmyo, and M. Horiguchi, "Erbium-doped silica-based planar waveguide amplifier pumped by $0.98 \mu \mathrm{m}$ laser diodes," Electronics Letters 29, 357 (1993).

[46] K. Shuto, K. Hattori, T. Kitagawa, Y. Ohmori, and M. Horiguchi, "Erbium-doped phosphosilicate glass waveguide amplifier fabricated by PECVD," Electronics Letters 29, 139 (1993).

[47] G. N. van den Hoven, R. J. I. M. Koper, A. Polman, C. van Dam, J. W. M. van Uffelen, and M. K. Smit, "Net optical gain at $1.53 \mu \mathrm{m}$ in Er-doped $\mathrm{Al}_{2} \mathrm{O}_{3}$ waveguides on silicon," Applied Physics Letters 68, 1886 (1996).

[48] Y. C. Yan, A. J. Faber, H. de Waal, P. G. Kik, and A. Polman, "Erbium-doped phosphate glass waveguide on silicon with $4.1 \mathrm{~dB} / \mathrm{cm}$ gain at $1.535 \mu \mathrm{m}, "$ Applied Physics Letters 71, 2922 (1997).

[49] K. Vu and S. Madden, "Tellurium dioxide erbium doped planar rib waveguide amplifiers with net gain and $2.8 \mathrm{~dB} / \mathrm{cm}$ internal gain," Optics Express 18, 19192 (2010).

[50] K. Vu, S. Farahani, and S. Madden, "980 nm pumped erbium doped tellurium oxide planar rib waveguide laser and amplifier with gain in S, C and L band," Optics Express 23, 747 (2015).

[51] H. Isshiki, F. Jing, T. Sato, T. Nakajima, and T. Kimura, "Rare earth silicates as gain media for silicon photonics," Photonics Research 2, A45 (2014).

[52] F. D. Patel, S. DiCarolis, P. Lum, S. Venkatesh, and J. N. Miller, "A compact high-performance optical waveguide amplifier," IEEE Photonics Technology Letters 16, 2607 (2004).

[53] B.-C. Hwang, S. Jiang, T. Luo, J. Watson, G. Sorbello, and N. Peyghambarian, "Cooperative upconversion and energy transfer of new high $\mathrm{Er}^{3+}$ - and $\mathrm{Yb}^{3+}-\mathrm{Er}^{3+}$ doped phosphate glasses," Journal of the Optical Society of America B 17, 833 (2000). 
[54] G. A. Sefler, W. D. Mack, G. C. Valley, and T. S. Rose, "Secondary energy transfer and nonparticipatory $\mathrm{Yb}^{3+}$ ions in $\mathrm{Er}^{3+}-\mathrm{Yb}^{3+}$ high-power amplifier fibers," Journal of the Optical Society of America B 21, 1740 (2004).

[55] J. Yang, M. B. J. Diemeer, D. Geskus, G. Sengo, M. Pollnau, and A. Driessen, "Neodymium-complex-doped photodefined polymer channel waveguide amplifiers," Optics Letters 34, 473 (2009).

[56] J. Yang, M. B. J. Diemeer, G. Sengo, M. Pollnau, and A. Driessen, "Nd-doped polymer waveguide amplifiers," IEEE Journal of Quantum Electronics 46, 1043 (2010).

[57] J. Yang, T. Lamprecht, K. Wörhoff, A. Driessen, F. Horst, B. J. Offrein, F. Ay, and M. Pollnau, "Integrated optical backplane amplifier," IEEE Journal of Selected Topics in Quantum Electronics 17, 609 (2011).

[58] Y. Tan, Q. Luan, F. Liu, S. Akhmadaliev, S. Zhou, and F. Chen, "Swift carbon ion irradiated Nd:YAG ceramic optical waveguide amplifier," Optics Express 21, 13992 (2013).

[59] A. Aghajani, G. S. Murugan, N. P. Sessions, S. J. Pearce, V. Apostolopoulos, and J. S. Wilkinson, "Spectroscopy of ytterbium-doped tantalum pentoxide rib waveguides on silicon," Optical Materials Express 4, 1505 (2014).

[60] A. Aznar, R. Solé, M. Aguiló, F. Diaz, U. Griebner, R. Grunwald, and V. Petrov, "Growth, optical characterization, and laser operation of epitaxial $\mathrm{Yb}: \mathrm{KY}\left(\mathrm{WO}_{4}\right)_{2} \mathrm{KY}\left(\mathrm{WO}_{4}\right)_{2}$ composites with monoclinic structure," Applied Physics Letters 85, 4313 (2004).

[61] Y. E. Romanyuk, I. Utke, D. Ehrentraut, V. Apostolopoulos, M. Pollnau, S. García-Revilla, and R. Valiente, "Low-temperature liquid-phase epitaxy and optical waveguiding of rare-earth-ion-doped $\mathrm{KY}\left(\mathrm{WO}_{4}\right)_{2}$ thin layers," Journal of Crystal Growth 269, 377 (2004).

[62] Y. E. Romanyuk, C. N. Borca, M. Pollnau, S. Rivier, V. Petrov, and U. Griebner, "Yb-doped KY $\left(\mathrm{WO}_{4}\right)_{2}$ planar waveguide laser," Optics Letters 31, 53 (2006).

[63] M. Pollnau, Y. E. Romanyuk, F. Gardillou, C. N. Borca, U. Griebner, S. Rivier, and V. Petrov, "Double tungstate lasers: From bulk toward on-chip integrated waveguide devices," IEEE Journal of Selected Topics in Quantum Electronics 13, 661 (2007).

[64] F. Gardillou, Y. E. Romanyuk, C. N. Borca, R.-P. Salathé, and M. Pollnau, "Lu, Gd codoped $\mathrm{KY}\left(\mathrm{WO}_{4}\right)_{2}: \mathrm{Yb}$ epitaxial layers: towards integrated optics based on $\mathrm{KY}\left(\mathrm{WO}_{4}\right)_{2}$," Optics Letters 32, 488 (2007).

[65] S. Aravazhi, D. Geskus, K. van Dalfsen, S. A. Vázquez-Córdova, C. Grivas, U. Griebner, S. M. García-Blanco, and M. Pollnau, "Engineering lattice matching, doping level, and optical properties of $\mathrm{KY}\left(\mathrm{WO}_{4}\right)_{2}: \mathrm{Gd}, \mathrm{Lu}, \mathrm{Yb}$ layers for a cladding-side-pumped channel waveguide laser," Applied Physics B 111, 433 (2013).

[66] D. Geskus, S. Aravazhi, E. Bernhardi, C. Grivas, S. Harkema, K. Hametner, D. Günther, K. Wörhoff, and M. Pollnau, "Low-threshold, highly efficient $\mathrm{Gd}^{3+}$, $\mathrm{Lu}^{3+}$ co-doped $\mathrm{KY}\left(\mathrm{WO}_{4}\right)_{2}: \mathrm{Yb}^{3+}$ planar waveguide lasers," Laser Physics Letters 6, 800 (2009). 
[67] D. Geskus, S. Aravazhi, K. Wörhoff, and M. Pollnau, "High-power, broadly tunable, and low-quantum-defect $\mathrm{KGd}_{1-\mathrm{x}} \mathrm{Lu}_{\mathrm{x}}\left(\mathrm{WO}_{4}\right)_{2}: \mathrm{Yb}^{3+}$ channel waveguide lasers," Optics Express 18, 26107 (2010).

[68] K. van Dalfsen, S. Aravazhi, C. Grivas, S. M. Garcia-Blanco, and M. Pollnau, "Thulium channel waveguide laser with $1.6 \mathrm{~W}$ of output power and approximately 80\% slope efficiency," Optics Letters 39, 4380 (2014).

[69] S. A. Vázquez-Córdova, S. Aravazhi, C. Grivas, Y. S. Yong, S. M. GarcíaBlanco, J. L. Herek, and M. Pollnau, "13 dB/cm gain per unit length at $1.53 \mu \mathrm{m}$ in $\mathrm{KGd}_{\mathrm{x}} \mathrm{Lu}_{\mathrm{y}} \mathrm{Er}_{1-\mathrm{x}-\mathrm{y}}\left(\mathrm{WO}_{4}\right)_{2}$ channel waveguides, allowing for $\sim 40 \mathrm{~dB}$ total gain," in Advanced Solid State Lasers 2016, OSA Technical Digest (Optical Society of America, 2016), ATu6A.3.

[70] J. Koerner, C. Vorholt, H. Liebetrau, M. Kahle, D. Kloepfel, R. Seifert, J. Hein, and M. C. Kaluza, "Measurement of temperature-dependent absorption and emission spectra of $\mathrm{Yb}: \mathrm{YAG}, \mathrm{Yb}: \mathrm{LuAG}$, and $\mathrm{Yb}: \mathrm{CaF}_{2}$ between $20{ }^{\circ} \mathrm{C}$ and $200{ }^{\circ} \mathrm{C}$ and predictions on their influence on laser performance," Journal of the Optical Society of America B 29, 2493 (2012).

[71] N. V. Kuleshov, A. A. Lagatsky, A. V. Podlipensky, V. P. Mikhailov, and G. Huber, "Pulsed laser operation of $\mathrm{Yb}$-doped $\mathrm{KY}\left(\mathrm{WO}_{4}\right)_{2}$ and $\mathrm{KGd}\left(\mathrm{WO}_{4}\right)_{2}$," Optics Letters 22, 1317 (1997).

[72] M. C. Pujol, M. A. Bursukova, F. Güell, X. Mateos, R. Solé, J. Gavaldà, M. Aguiló, J. Massons, F. Díaz, P. Klopp, U. Griebner, and V. Petrov, "Growth, optical characterization, and laser operation of a stoichiometric crystal $\mathrm{KYb}\left(\mathrm{WO}_{4}\right)_{2}, "$ Physical Review B 65, 165121 (2002).

[73] X. Mateos, M. C. Pujol, F. Güell, M. Galán, R. M. Solé, J. Gavaldà, M. Aguiló, J. Massons, and F. Díaz, "Erbium spectroscopy and $1.5-\mu \mathrm{m}$ emission in $\mathrm{KGd}\left(\mathrm{WO}_{4}\right)_{2}$ : Er,Yb single crystals," IEEE Journal of Quantum Electronics 40, 759 (2004).

[74] X. Mateos, R. Solé, J. Gavaldà, M. Aguiló, J. Massons, F. Díaz, V. Petrov, and U. Griebner, "Crystal growth, spectroscopic studies and laser operation of $\mathrm{Yb}^{3+}$ doped potassium lutetium tungstate," Optical Materials 28, 519 (2006).

[75] A. Knigge, G. Erbert, J. Jönsson, W. Pittroff, R. Staske, B. Sumpf, M. Weyers, and G. Tränkle, "Passively cooled $940 \mathrm{~nm}$ laser bars with 73\% wall-plug efficiency at $70 \mathrm{~W}$ and $25^{\circ} \mathrm{C}$," Electronics Letters 41, 250 (2005).

[76] K. Toeda, "Do bacteria and DNA adsorb rare earth elements? Opening the door to application from atomic-level viewpoint," http://www.spring8.or.jp/en/news_publications/research_highlights/no_56/.

[77] S. Biswal, S. P. O'Connor, and S. R. Bowman, "Nonradiative losses in $\mathrm{Yb}: \mathrm{KGd}\left(\mathrm{WO}_{4}\right)_{2}$ and $\mathrm{Yb}: \mathrm{Y}_{3} \mathrm{Al}_{5} \mathrm{O}_{12}, "$ Applied Physics Letters 89, 091911 (2006).

[78] P. V. dos Santos, M. V. D. Vermelho, E. A. Gouveia, M. T. de Araújo, A. S. Gouveia-Neto, F. C. Cassanjes, S. J. L. Ribeiro, and Y. Messaddeq, "Blue cooperative luminescence in $\mathrm{Yb}^{3+}$-doped tellurite glasses excited at $1.064 \mu \mathrm{m}$," Journal of Chemical Physics 116, 6772 (2002). 
[79] W. T. Carnall, G. L. Goodman, K. Rajnak, and R. S. Rana, "A systematic analysis of the spectra of the lanthanides doped into single crystal $\mathrm{LaF}_{3}$ " The Journal of Chemical Physics 90, 3443 (1989).

[80] A. Einstein, "Zur Quantentheorie der Strahlung," Physikalische Zeitschrift 18, 121 (1917).

[81] B. E. A. Saleh and M. C. Teich, Fundamentals of photonics, 2nd ed. (WileyInterscience, 2007).

[82] V. E. Kisel, A. E. Troshin, V. G. Shcherbitskii, N. V. Kuleshov, A. A. Pavlyuk, F. Brunner, R. Paschotta, F. Morier-Genoud, and U. Keller, "Luminescent and lasing characteristics of heavily doped $\mathrm{Yb}^{3+}: \mathrm{KY}\left(\mathrm{WO}_{4}\right)_{2}$ crystals," Quantum Electronics 36, 319 (2006).

[83] K. Petermann, D. Fagundes-Peters, J. Johannsen, M. Mond, V. Peters, J. J. Romero, S. Kutovoi, J. Speiser, and A. Giesen, "Highly Yb-doped oxides for thin-disc lasers," Journal of Crystal Growth 275, 135 (2005).

[84] M. Eichhorn, S. T. Fredrich-Thornton, E. Heumann, and G. Huber, "Spectroscopic properties of $\mathrm{Er}^{3+}$ :YAG at $300-550 \mathrm{~K}$ and their effects on the $1.6 \mu \mathrm{m}$ laser transitions," Applied Physics B 91, 249 (2008).

[85] Y. Sato and T. Taira, "Temperature dependencies of stimulated emission cross section for Nd-doped solid-state laser materials," Optical Materials Express 2, 1076 (2012).

[86] D. E. McCumber, "Einstein relations connecting broadband emission and absorption spectra," Physical Review 136, A954 (1964).

[87] S. A. Payne, L. L. Chase, L. K. Smith, W. L. Kway, and W. F. Krupke, "Infrared cross-section measurements for crystals doped with $\mathrm{Er}^{3+}, \mathrm{Tm}^{3+}$, and $\mathrm{Ho}^{3+}$, "IEEE Journal of Quantum Electronics 28, 2619 (1992).

[88] W. B. Fowler and D. L. Dexter, "Relation between absorption and emission probabilities in luminescent centers in ionic solids," Physical Review 128, 2154 (1962).

[89] M. Eichhorn and M. Pollnau, "Spectroscopic foundations of lasers: Spontaneous emission into a resonator mode," IEEE Journal of Selected Topics in Quantum Electronics 21, 9000216 (2015).

[90] C. Füchtbauer, G. Joos, and O. Dinkelacker, "Über Intensität, Verbreiterung und Druckverschiebung von Spektrallinien, insbesondere der Absorptionslinie 2537 des Quecksilbers," Ann. Phys.-Leipzig, Band 376 - Vierte Folge 71, 204 (1923).

[91] R. Ladenburg, "Die quantentheoretische Deutung der Zahl der Dispersionselektronen," Z. Phys. 4, 451 (1921).

[92] A. A. Demidovich, A. N. Kuzmin, G. I. Ryabtsev, M. B. Danailov, W. Strek, and A. N. Titov, "Influence of $\mathrm{Yb}$ concentration on $\mathrm{Yb}: \mathrm{KYW}$ laser properties," Journal of Alloys and Compounds 300-301, 238 (2000).

[93] D. S. Sumida and T. Y. Fan, "Effect of radiation trapping on fluorescence lifetime and emission cross section measurements in solid-state laser media," Optics Letters 19, 1343 (1994).

[94] H. Kühn, K. Petermann, and G. Huber, "Correction of reabsorption artifacts in fluorescence spectra by the pinhole method," Optics Letters 35, 1524 (2010). 
[95] K. Beil, S. T. Fredrich-Thornton, F. Tellkamp, R. Peters, C. Kränkel, K. Petermann, and G. Huber, "Thermal and laser properties of $\mathrm{Yb}: \mathrm{LuAG}$ for $\mathrm{kW}$ thin disk lasers," Optics Express 18, 20712 (2010).

[96] D. P. Shepherd, S. J. Hettrick, C. Li, J. I. Mackenzie, R. J. Beach, S. C. Mitchell, and H. E. Meissner, "High-power planar dielectric waveguide lasers," Journal of Physics D: Applied Physics 34, 2420 (2001).

[97] F. M. Bain, A. A. Lagatsky, R. R. Thomson, N. D. Psaila, N. V. Kuleshov, A. K. Kar, W. Sibbett, and C. T. A. Brown, "Ultrafast laser inscribed Yb:KGd( $\left(\mathrm{WO}_{4}\right)_{2}$ and $\mathrm{Yb}: \mathrm{KY}\left(\mathrm{WO}_{4}\right)_{2}$ channel waveguide lasers," Optics Express 17, 22417 (2009).

[98] C. N. Borca, V. Apostolopoulos, F. Gardillou, H. G. Limberger, M. Pollnau, and R. P. Salathé, "Buried channel waveguides in Yb-doped $\mathrm{KY}\left(\mathrm{WO}_{4}\right)_{2}$ crystals fabricated by femtosecond laser irradiation," Applied Surface Science 253, 8300 (2007).

[99] D. Geskus, S. Aravazhi, C. Grivas, K. Wörhoff, and M. Pollnau, "Microstructured $\mathrm{KY}\left(\mathrm{WO}_{4}\right)_{2}: \mathrm{Gd}^{3+}, \mathrm{Lu}^{3+}, \mathrm{Yb}^{3+}$ channel waveguide laser," Optics Express 18, 8853 (2010).

[100] W. Bolaños, J. J. Carvajal, X. Mateos, G. S. Murugan, A. Z. Subramanian, J. S. Wilkinson, E. Cantelar, D. Jaque, G. Lifante, M. Aguilo, and F. Diaz, "Mirrorless buried waveguide laser in monoclinic double tungstates fabricated by a novel combination of ion milling and liquid phase epitaxy," Optics Express 18, 26937 (2010).

[101] K. M. Davis, K. Miura, N. Sugimoto, and K. Hirao, "Writing waveguides in glass with a femtosecond laser," Optics Letters 21, 1729 (1996).

[102] F. M. Bain, "Yb:tungstate waveguide lasers," Ph.D. Thesis, University of St Andrews (2010).

[103] A. A. Kaminskii, A. F. Konstantinova, V. P. Orekhova, A. V. Butashin, R. F. Klevtsova, and A. A. Pavlyuk, "Optical and nonlinear laser properties of the $\chi^{(3)}$ active monoclinic $\alpha-\mathrm{KY}\left(\mathrm{WO}_{4}\right)_{2}$ crystals," Crystallography Reports 46, 665 (2001).

[104] M. C. Pujol, X. Mateos, R. Solé, J. Massons, J. Gavaldà, X. Solans, F. Díaz, and M. Aguiló, "Structure, crystal growth and physical anisotropy of $\mathrm{KYb}\left(\mathrm{WO}_{4}\right)_{2}$, a new laser matrix," Journal of Applied Crystallography 35, 108 (2002).

[105] X. Mateos, R. Solé, J. Gavaldà, M. Aguiló, J. Massons, and F. Díaz, "Crystal growth, optical and spectroscopic characterisation of monoclinic $\mathrm{KY}\left(\mathrm{WO}_{4}\right)_{2}$ codoped with $\mathrm{Er}^{3+}$ and $\mathrm{Yb}^{3+}$," Optical Materials 28, 423 (2006).

[106] P. Klopp, U. Griebner, V. Petrov, X. Mateos, M. A. Bursukova, M. C. Pujol, R. Sole, J. Gavalda, M. Aguilo, F. Güell, J. Massons, T. Kirilov, and F. Diaz, "Laser operation of the new stoichiometric crystal $\mathrm{KYb}\left(\mathrm{WO}_{4}\right)_{2}$," Applied Physics B 74, 185 (2002).

[107] V. Petrov, M. C. Pujol, X. Mateos, Ò. Silvestre, S. Rivier, M. Aguiló, R. M. Solé, J. Liu, U. Griebner, and F. Díaz, "Growth and properties of $\mathrm{KLu}\left(\mathrm{WO}_{4}\right)_{2}$, and novel ytterbium and thulium lasers based on this monoclinic crystalline host," Laser \& Photonics Review 1, 179 (2007). 
[108] A. Rudenkov, V. Kisel, A. Yasukevich, K. Hovhannesyan, A. Petrosyan, and N. Kuleshov, "Spectroscopy and continuous wave laser performance of $\mathrm{Yb}^{3+}: \mathrm{LuAlO}_{3}$ crystal," Optics Letters 41, 5805 (2016).

[109] M. C. Pujol, X. Mateos, R. Solé, J. Gavaldà, J. Massons, M. Aguiló, and F. Díaz, "Linear thermal expansion tensor in $\mathrm{KRE}\left(\mathrm{WO}_{4}\right)_{2}(\mathrm{RE}=\mathrm{Gd}, \mathrm{Y}, \mathrm{Er}, \mathrm{Yb})$ monoclinic crystals," Materials Science Forum 378-381, 710 (2001).

[110] P. A. Loiko, K. V. Yumashev, N. V. Kuleshov, and A. A. Pavlyuk, "Thermooptical properties of pure and $\mathrm{Yb}$-doped monoclinic $\mathrm{KY}\left(\mathrm{WO}_{4}\right)_{2}$ crystals," Applied Physics B 106, 663 (2011).

[111] M. C. Pujol, R. Solé, J. Massons, J. Gavaldà, X. Solans, C. Zaldo, F. Díaz, and M. Aguiló, "Structural study of monoclinic $\mathrm{KGd}\left(\mathrm{WO}_{4}\right)_{2}$ and effects of lanthanide substitution," Journal of Applied Crystallography 34, 1 (2001).

[112] M. C. Pujol, X. Mateos, A. Aznar, X. Solans, S. Suriñach, J. Massons, F. Díaz, and M. Aguiló, "Structural redetermination, thermal expansion and refractive indices of $\mathrm{KLu}\left(\mathrm{WO}_{4}\right)_{2}$," Journal of Applied Crystallography 39, 230 (2006).

[113] M. C. Pujol, M. Rico, C. Zaldo, R. Solé, V. Nikolov, X. Solans, M. Aguiló, and F. Díaz, "Crystalline structure and optical spectroscopy of $\mathrm{Er}^{3+}$-doped $\mathrm{KGd}\left(\mathrm{WO}_{4}\right)_{2}$ single crystals," Applied Physics B 68, 187 (1999).

[114] S. Biswal, S. P. O'Connor, and S. R. Bowman, "Thermo-optical parameters measured in ytterbium-doped potassium gadolinium tungstate," Applied Optics 44, 3093 (2005).

[115] Ò. Silvestre, J. Grau, M. C. Pujol, J. Massons, M. Aguiló, F. Díaz, M. T. Borowiec, A. Szewczyk, M. U. Gutowska, M. Massot, A. Salazar, and V. Petrov, "Thermal properties of monoclinic $\mathrm{KLu}\left(\mathrm{WO}_{4}\right)_{2}$ as a promising solid state laser host," Optics Express 16, 5022 (2008).

[116] J. Zhang, K. Wang, J. Wang, H. Zhang, W. Yu, X. Wang, Z. Wang, Q. Lu, M. Ba, D. G. Ran, Z. C. Ling, and H. R. Xia, "Anisotropic thermal properties of monoclinic $\mathrm{Yb}: \mathrm{KLu}\left(\mathrm{WO}_{4}\right)_{2}$ crystals," Applied Physics Letters 87, 061104 (2005).

[117] S. Chénais, F. Druon, S. Forget, F. Balembois, and P. Georges, "On thermal effects in solid-state lasers: The case of ytterbium-doped materials," Progress in Quantum Electronics 30, 89 (2006).

[118] R. L. Aggarwal, D. J. Ripin, J. R. Ochoa, and T. Y. Fan, "Measurement of thermo-optic properties of $\mathrm{Y}_{3} \mathrm{Al}_{5} \mathrm{O}_{12}, \mathrm{Lu}_{3} \mathrm{Al}_{5} \mathrm{O}_{12}, \mathrm{YAlO}_{3}, \mathrm{LiYF}_{4}, \mathrm{LiLuF}_{4}, \mathrm{BaY}_{2} \mathrm{~F}_{8}$, $\mathrm{KGd}\left(\mathrm{WO}_{4}\right)_{2}$, and $\mathrm{KY}\left(\mathrm{WO}_{4}\right)_{2}$ laser crystals in the 80-300 K temperature range," Journal of Applied Physics 98, 103514 (2005).

[119] H. Qiu, P. Yang, J. Dong, P. Deng, J. Xu, and W. Chen, "The influence of Yb concentration on laser crystal Yb:YAG," Materials Letters 55, 1 (2002).

[120] F. D. Patel, E. C. Honea, J. Speth, S. A. Payne, R. Hutcheson, and R. Equall, "Laser demonstration of $\mathrm{Yb}_{3} \mathrm{Al}_{5} \mathrm{O}_{12}$ (YbAG) and materials properties of highly doped Yb:YAG," IEEE Journal of Quantum Electronics 37, 135 (2001).

[121] W. Bolaños, J. J. Carvajal, X. Mateos, M. Aguiló, and F. Díaz, "Exploring waveguiding properties of heavily doped $\mathrm{Yb}^{3+}: \mathrm{KLu}\left(\mathrm{WO}_{4}\right)_{2}$ epitaxial layers," IEEE Photonics Journal 2, 482 (2010). 
[122] O. Silvestre, M. C. Pujol, R. Solé, W. Bolaños, J. J. Carvajal, J. Massons, M. Aguiló, and F. Diaz, "Ln ${ }^{3+}: \mathrm{KLu}\left(\mathrm{WO}_{4}\right)_{2} / \mathrm{KLu}\left(\mathrm{WO}_{4}\right)_{2}$ epitaxial layers: Crystal growth and physical characterisation," Materials Science and Engineering: $B$ 146, 59 (2008).

[123] N. V. Kuleshov, A. A. Lagatsky, V. G. Shcherbitsky, V. P. Mikhailov, E. Heumann, T. Jensen, A. Diening, and G. Huber, "CW laser performance of $\mathrm{Yb}$ and Er,Yb doped tungstates," Applied Physics B: Lasers and Optics 64, 409 (1997).

[124] O. Silvestre, A. Aznar, R. Solé, M. C. Pujol, F. Díaz, and M. Aguiló, "Lattice mismatch and crystal growth of monoclinic $\mathrm{KY}_{1-\mathrm{x}} \mathrm{Yb}_{\mathrm{x}}\left(\mathrm{WO}_{4}\right)_{2} / \mathrm{KY}\left(\mathrm{WO}_{4}\right)_{2}$ layers by liquid phase epitaxy," Journal of Physics: Condensed Matter 20, 225004 (2008).

[125] S. Bjurshagen, J. E. Hellström, V. Pasiskevicius, M. C. Pujol, M. Aguiló, and F. Díaz, "Fluorescence dynamics and rate equation analysis in $\mathrm{Er}^{3+}$ and $\mathrm{Yb}^{3+}$ doped double tungstates," Applied Optics 45, 4715 (2006).

[126] V. E. Kisel, A. E. Troshin, V. G. Shcherbitsky, and N. V. Kuleshov, "Luminescence lifetime measurements in $\mathrm{Yb}^{3+}$-doped $\mathrm{KY}\left(\mathrm{WO}_{4}\right)_{2}$ and $\mathrm{KGd}\left(\mathrm{WO}_{4}\right)_{2}$," in Advanced Solid-State Photonics 2004, OSA Technical Digest (Optical Society of America, 2004), WB7.

[127] B. Jacobsson, "Experimental and theoretical investigation of a volume-Bragggrating-locked Yb:KYW laser at selected wavelengths," Optics Express 16, 6443 (2008).

[128] X. Xu, Z. Zhao, P. Song, G. Zhou, J. Xu, and P. Deng, "Structural, thermal, and luminescent properties of $\mathrm{Yb}$-doped $\mathrm{Y}_{3} \mathrm{Al}_{5} \mathrm{O}_{12}$ crystals," Journal of the Optical Society of America B 21, 543 (2004).

[129] D.-L. Zhang and E. Y. B. Pun, "Accurate measurement of $1.5 \mu \mathrm{m}$ lifetime of $\mathrm{Er}^{3+}$ in $\mathrm{LiNbO}_{3}$ crystals and waveguides," Journal of Applied Physics 94, 1339 (2003).

[130] V. E. Kisel, A. E. Troshin, N. A. Tolstik, V. G. Shcherbitsky, N. V. Kuleshov, V. N. Matrosov, T. A. Matrosova, and M. I. Kupchenko, "Spectroscopy and continuous-wave diode-pumped laser action of $\mathrm{Yb}^{3+}: \mathrm{YVO}_{4}, "$ Optics Letters 29, 2491 (2004).

[131] V. E. Kisel, S. V. Kurilchik, A. S. Yasukevich, S. V. Grigoriev, S. A. Smirnova, and N. V. Kuleshov, "Spectroscopy and femtosecond laser performance of $\mathrm{Yb}^{3+}: \mathrm{YAlO}_{3}$ crystal," Optics Letters 33, 2194 (2008).

[132] H. Kühn, S. T. Fredrich-Thornton, C. Kränkel, R. Peters, and K. Petermann, "Model for the calculation of radiation trapping and description of the pinhole method," Optics Letters 32, 1908 (2007).

[133] C. Kränkel, D. Fagundes-Peters, S. T. Fredrich, J. Johannsen, M. Mond, G. Huber, M. Bernhagen, and R. Uecker, "Continuous wave laser operation of $\mathrm{Yb}^{3+}: \mathrm{YVO}_{4}, "$ Applied Physics B 79, 543 (2004).

[134] S. Guy, "Modelization of lifetime measurement in the presence of radiation trapping in solid-state materials," Physical Review B 73, 144101 (2006). 
[135] K. P. Ghiggino, M. R. Harris, and P. G. Spizzirri, "Fluorescence lifetime measurements using a novel fiber-optic laser scanning confocal microscope," Review of Scientific Instruments 63, 2999 (1992).

[136] P. Brynolfsson, "Accurate lifetime measurements using confocal microscopy," Master Thesis, Kungliga Tekniska Högskolan (2007).

[137] F. Auzel, F. Bonfigli, S. Gagliari, and G. Baldacchini, "The interplay of selftrapping and self-quenching for resonant transitions in solids; role of a cavity," Journal of Luminescence 94-95, 293 (2001).

[138] W. J. C. Grant, "Role of rate equations in the theory of luminescent energy transfer," Physical Review B 4, 648 (1971).

[139] P. S. Golding, S. D. Jackson, T. A. King, and M. Pollnau, "Energy transfer processes in $\mathrm{Er}^{3+}$-doped and $\mathrm{Er}^{3+}, \mathrm{Pr}^{3+}$-codoped ZBLAN glasses," Physical Review B 62, 856 (2000).

[140] D. Fagundes-Peters, N. Martynyuk, K. Lünstedt, V. Peters, K. Petermann, G. Huber, S. Basun, V. Laguta, and A. Hofstaetter, "High quantum efficiency YbAG-crystals," Journal of Luminescence 125, 238 (2007).

[141] D. Luo, J. Zhang, C. Xu, H. Yang, H. Lin, H. Zhu, and D. Tang, "Yb:LuAG laser ceramics: a promising high power laser gain medium," Optical Materials Express 2, 1425 (2012).

[142] F. Auzel, "Upconversion processes in coupled ion systems," Journal of Luminescence 45, 341 (1990).

[143] Y. Sato and T. Taira, "Saturation factors of pump absorption in solid-state lasers," IEEE Journal of Quantum Electronics 40, 270 (2004).

[144] P. Klopp, "New $\mathrm{Yb}^{3+}$-doped laser materials and their application in continuouswave and mode-locked lasers," Ph.D. Thesis, Humboldt-Universität (2006).

[145] M. R. Sharpe, "Stray light in UV-VIS spectrophotometers," Analytical Chemistry 56, 339 (1984).

[146] A. Major, I. Nikolakakos, J. S. Aitchison, A. I. Ferguson, N. Langford, and P. W. E. Smith, "Characterization of the nonlinear refractive index of the laser crystal Yb:KGd((WO $)_{2}$," Applied Physics B 77, 433 (2003).

[147] P. Loiko, S. J. Yoon, J. M. Serres, X. Mateos, S. J. Beecher, R. B. Birch, V. G. Savitski, A. J. Kemp, K. Yumashev, U. Griebner, V. Petrov, M. Aguiló, F. Díaz, and J. I. Mackenzie, "Temperature-dependent spectroscopy and microchip laser operation of $\mathrm{Nd}: \mathrm{KGd}\left(\mathrm{WO}_{4}\right)_{2}$," Optical Materials 58, 365 (2016).

[148] I. V. Mochalov, "Laser and nonlinear properties of the potassium gadolinium tungstate laser crystal $\mathrm{KGd}\left(\mathrm{WO}_{4}\right)_{2}: \mathrm{Nd}^{3+}-(\mathrm{KGW}: \mathrm{Nd}), "$ Optical Engineering 36, 1660 (1997).

[149] G. G. Demirkhanyan, H. G. Demirkhanyan, E. P. Kokanyan, R. B. Kostanyan, J. B. Gruber, K. L. Nash, and D. K. Sardar, "Phonon effects on zero-phonon transitions between Stark levels in $\mathrm{NaBi}\left(\mathrm{WO}_{4}\right)_{2}: \mathrm{Yb}^{3+}, "$ Journal of Applied Physics 105, 063106 (2009).

[150] G. G. Demirkhanyan and R. B. Kostanyan, "Temperature dependence of spectralline intensities in YAG: $\mathrm{Yb}^{3+}, "$ Laser Physics 18, 104 (2011). 
[151] M. Larionov, K. Schuhmann, J. Speiser, C. Stolzenburg, and A. Giesen, "Nonlinear decay of the excited state in Yb:YAG," in Advanced Solid-State Photonics 2005, Technical Digest (Optical Society of America, 2005), TuB49.

[152] R. Paschotta, J. Nilsson, P. R. Barber, J. E. Caplen, A. C. Tropper, and D. C. Hanna, "Lifetime quenching in Yb-doped fibres," Optics Communications 136, 375 (1997).

[153] Z. Burshtein, Y. Kalisky, S. Z. Levy, P. Le Boulanger, and S. Rotman, "Impurity local phonon nonradiative quenching of $\mathrm{Yb}^{3+}$ fluorescence in ytterbium-doped silicate glasses," IEEE Journal of Quantum Electronics 36, 1000 (2000).

[154] S. Banerjee, C. C. Baker, A. J. Steckl, and D. Klotzkin, "Optical properties of Er in Er-doped $\mathrm{Zn}_{2} \mathrm{Si}_{0.5} \mathrm{Ge}_{0.5} \mathrm{O}_{4}$ waveguide amplifiers," Journal of Lightwave Technology 23, 1342 (2005).

[155] R. Paschotta, J. Nilsson, A. C. Tropper, and D. C. Hanna, "Ytterbium-doped fiber amplifiers," IEEE Journal of Quantum Electronics 33, 1049 (1997).

[156] M. E. Innocenzi, H. T. Yura, C. L. Fincher, and R. A. Fields, "Thermal modeling of continuous - wave end - pumped solid - state lasers," Applied Physics Letters 56, 1831 (1990).

[157] P. A. Loiko, K. V. Yumashev, N. V. Kuleshov, and A. A. Pavlyuk, "Comparative thermal analysis of $\mathrm{Nd}$ - and $\mathrm{Yb}$-doped $\mathrm{YAG}$ and $\mathrm{KGdW}$ laser crystals under diode- and flashlamp-pumping," Optics \& Laser Technology 44, 2232 (2012).

[158] A. K. Cousins, "Temperature and thermal stress scaling in finite-length endpumped laser rods," IEEE Journal of Quantum Electronics 28, 1057 (1992).

[159] S. Chénais, F. Balembois, F. Druon, G. Lucas-Leclin, and P. Georges, "Thermal lensing in diode-pumped ytterbium lasers-Part I: theoretical analysis and wavefront measurements," IEEE Journal of Quantum Electronics 40, 1217 (2004).

[160] S. Chénais, S. Forget, F. Druon, F. Balembois, and P. Georges, "Direct and absolute temperature mapping and heat transfer measurements in diode-endpumped Yb:YAG," Applied Physics B 79, 221 (2004).

[161] A. N. Kuzmin, A. V. Kachynski, P. N. Prasad, A. A. Demidovich, L. E. Batay, A. Bednarkiewicz, W. Strek, and A. N. Titov, "Blue up-conversion emission in Yband Tm-codoped potassium yttrium tungstate," Journal of Applied Physics 95, 7862 (2004).

[162] X. Mateos, R. Solé, J. Gavaldà, M. Aguiló, F. Díaz, and J. Massons, "Ultraviolet and visible emissions of $\mathrm{Er}^{3+}$ in $\mathrm{KY}\left(\mathrm{WO}_{4}\right)_{2}$ single crystals co-doped with $\mathrm{Yb}^{3+}$ ions," Journal of Luminescence 115, 131 (2005).

[163] Y. Guyot, A. Steimacher, M. P. Belançon, A. N. Medina, M. L. Baesso, S. M. Lima, L. H. C. Andrade, A. Brenier, A.-M. Jurdyc, and G. Boulon, "Spectroscopic properties, concentration quenching, and laser investigations of $\mathrm{Yb}^{3+}$-doped calcium aluminosilicate glasses," Journal of the Optical Society of America B 28, 2510 (2011).

[164] A. Pirri, G. Toci, D. Alderighi, and M. Vannini, "Effects of the excitation density on the laser output of two differently doped Yb:YAG ceramics," Optics Express 18, 17262 (2010). 
[165] A. Pirri, G. Toci, M. Nikl, V. Babin, and M. Vannini, "Experimental evidence of a nonlinear loss mechanism in highly doped Yb:LuAG crystal," Optics Express 22, 4038 (2014).

[166] C. Brandt, S. T. Fredrich-Thornton, K. Petermann, and G. Huber, "Photoconductivity in Yb-doped oxides at high excitation densities," Applied Physics B 102, 765 (2011).

[167] U. Wolters, K. Beil, C. Krankel, K. Petermann, and G. Huber, "Temperature development in $\mathrm{Yb}$ :YAG thin-disk lasers at high inversion densities confirming nonlinear losses," in The European Conference on Lasers and Electro-Optics 2013, 2013 Conference on Lasers and Electro-Optics - International Quantum Electronics Conference (Optical Society of America, 2013), CA_8_5.

[168] K. Wörhoff, R. G. Heideman, A. Leinse, and M. Hoekman, "TriPleX: a versatile dielectric photonic platform," Advances in Optical Technologies 4, 189 (2015).

[169] W. Huang, R. R. A. Syms, E. M. Yeatman, M. M. Ahmad, T. V. Clapp, and S. M. Ojha, "Fiber-device-fiber gain from a sol-gel erbium-doped waveguide amplifier," IEEE Photonics Technology Letters 14, 959 (2002). 



\section{Acknowledgements}

My PhD journey, which began in February 2012, has been a great experience. I am fortunate to be surrounded by colleagues, friends, and family who support me along the journey, without whom this thesis would not have been possible.

I am deeply grateful to my supervisor Prof. Dr. Markus Pollnau for offering me the opportunity to work on the IWAOI project under his supervision. I thank him for the guidance, for the patience in correcting my manuscripts, and for being available for technical discussion when needed, especially during the final stage of thesis writing. I wish to thank my other supervisor, Prof. Dr. Jennifer L. Herek, for her continual encouragement ("you're almost there!") and support, as well as the generous contract extension during the fifth year of my $\mathrm{PhD}$. I would like to express my gratitude to Assoc. Prof. Dr. Sonia M. García-Blanco for her daily supervision and for her kind extension of contract which allowed me to complete the thesis without unnecessary distractions. Special thanks go to Dr. Kerstin Wörhoff for her guidance during the initial stage of my PhD study.

I would also like to thank the rest of my graduation committee members: Prof. Dr. Cornelia Denz, Assoc. Prof. Dr. Joan J. Carvajal, Prof. Dr. Klaus Boller, and Prof. Dr. Lis Nanver for their valuable time and effort in reviewing this thesis.

I am lucky to have Sergio A. Vázquez-Córdova and Lantian Chang going through ups and downs of the $\mathrm{PhD}$ journey together over the past few years. Meneer Sergio has a remarkable skill of fixing anything in the laboratory and he is always ready to help whenever I face difficulty. He is the sifu who taught me measurement techniques and many other useful know-hows in the laboratory. Muchisimas gracias! Lantian and me often spent time discussing about anything related to "waveguide" and/or "optics" and I learnt a lot through these discussions. He always gives great advice at work and shares practical tips for daily life with me. Xie xie, Lantian!

I would like to express my appreciation to the members of Integrated Optical MicroSystems (IOMS) group. Thanks to Abu (Dr. Shanmugam Aravazhi) for growing the nice epitaxial layers. It was a great pleasure to discuss about a wide range of topics with Abu while polishing the precious epi-layers (which took long hours) during the first year of my PhD. I wish to thank Meindert Dijkstra (who keeps impressing me with creative workarounds for my special requests) for the assistance on cleanroom activities, Dimitri Geskus for sharing his experience on $\mathrm{KRE}\left(\mathrm{WO}_{4}\right)_{2}$ amplifiers, and Marko van Dalfsen for showing me the laser experiment (and for not being angry with me when I damaged his pump diode laser). Thanks to the secretary, Brigit Binkhorst- 
Reinshagen, for picking me up from the train station and arranging my accommodation when I first arrived in Enschede. I am thankful to Markus, Sonia, Dr. Hugo J. W. M. Hoekstra and Dr. René M. de Ridder for their guidance when I was working as teaching assistant in their courses. I thank other fellow IOMS members, Dr. Manfred Hammer, Anton Hollink, Gabriel Sengo, Mustafa A. Sefünç, Nur Ismail, Fehmi Çivitci, Edward H. Bernhardi, Laura Agazzi, Imran Akça, So Van Pham, Raghavendran T. Eachambadi, and Ron Hendriks for their help/advice and for creating an enjoyable working environment.

The members of the Optical Sciences (OS) group had also rendered their support during the second half of my PhD study. I would like to thank Dr. Ir. Herman L. Offerhaus for the discussion on confocal setup, which led to successful realization of confocal lifetime measurement setup within a short time. I am very grateful for the first class technical support provided by Ing. Jeroen Korterik and Ing. Frans Segerik especially during the transition period. Thanks to Dirk Jan Dikken and Qing Pan for their advice on measurement setup and luminescence lifetime extraction procedure. I thank Karen Munnink for handling all administrative matters efficiently. I appreciate the scientific discussions with the integrated Optical Sciences (iOS) subgroup members: Sergio, Meindert, Mustafa, Jinfeng Mu, Pablo Muñoz, Carlijn van Emmerik, Michiel de Goede, Simen Martinussen, Raimond Frentrop, Ward Hendriks, Marijn van de Putte, and Bahareh Sherafati. To the colleagues working together towards waveguide enhance Raman detection technique: Wooje Lee hyung (thanks for helping to build the setup), Pablo (thanks for the useful comments on Raman detection), Martijn Stok, Ivo Hegeman, and Peihua Liu, I wish you the best with your research endeavors. I thank the colleagues who shared the big office - the office which is equipped with (now broken) RC helicopters, (hidden) foam bullet blaster guns, and a door dedicated to height measurement (relative to your colleagues' height) - for cultivating a friendly and fun working atmosphere: Jorick van 't Oever (your Avogado plant is still surviving!), Dirk Jan, Gerwin Steen, Sean Stellingwerff, Rick Krabbendam (who surprised me with card tricks and Rubik's cube solving skill), Simon Huisman, Erik Garbacik, Sven van Binsbergen, Koen Dijkstra (who gave me light!), Raimond, Elena Beletkaia, Rob Lammerink, and Reinout Nonhebel. The abovementioned people, together with other OS members, including Dr. Ir. Annemarie Huijser, Sandra Drusova, Jesse Mak, Andrew Fussel, Ting-Li Chen (Dan), Joris de Graaf, Bente Elschot, and Tom Heidotting, made OS a great place to work. Thank you!

I would also like to thank the user committee of the IWAOI project: Ing. Jeroen Duis from TE connectivity, Dr. Bert J. Offrein from IBM Research, Dr. Jose Pozo, and Dr. Ir. Peter Harmsma from TNO, and Dr. Douwe Geuzebroek from Xio Photonics BV for their kind support on the project and valuable input during the project meetings. 
Many other colleagues from other departments had also contributed to the work in this thesis. I would like to thank Mark Smithers (EDX measurement) and Gerald Kip (XPS trials) from MESA+ as well as Hans Nijboer, Pieter Post, and Tom Buitenhuis from TCO (sample holders and polishing chucks) for their professionalism and excellent work.

Ying Du, Xichen Zhang, Youwen Fan, Siew Jean Goh, Caterina Taddei, Yin Tao, Marco Garcia Porcel, Rong Wang, Kuniyasu Saitoh, Megumi, Neru, thank you for your support and friendship during my stay in Enschede.

My ever supportive parents and siblings, thank you for standing behind me whenever I need and making it possible for me to persuade my $\mathrm{PhD}$ dream. Last but not least, to my wife Wai Ying, thank you for your unconditional love, patience and support. I am so blessed and thankful to have you by my side along this journey.

Thank you all, Yean-Sheng 



\section{List of publications}

\section{Peer-reviewed journal articles}

[1] Y. S. Yong, S. Aravazhi, S. A. Vázquez-Córdova, J. J. Carvajal, F. Díaz, J. L. Herek, S. M. García-Blanco, and M. Pollnau, "Optical gain in potassium double tungstate films with 57 at.\% and 76 at.\% ytterbium," in preparation (2017).

[2] Y. S. Yong, S. Aravazhi, S. A. Vázquez-Córdova, J. J. Carvajal, F. Díaz, J. L. Herek, S. M. García-Blanco, and M. Pollnau, "Direct confocal lifetime measurements on rare-earth-doped media exhibiting radiation trapping," Optical Materials Express, 7, 527 (2017).

[3] Y. S. Yong, S. Aravazhi, S. A. Vázquez-Córdova, J. J. Carvajal, F. Díaz, J. L. Herek, S. M. García-Blanco, and M. Pollnau, "Temperature-dependent absorption and emission of potassium double tungstates with high ytterbium content," Optics Express, 24, 26825 (2016).

[4] J. Mu, T. Alexoudi, Y. S. Yong, S. A. Vázquez-Córdova, M. Dijkstra, K. Wörhoff, J. Duis, and S. M. García-Blanco, "Low-loss highly tolerant flip-chip couplers for hybrid integration of $\mathrm{Si}_{3} \mathrm{~N}_{4}$ and polymer waveguides", IEEE Photonics Technology Letter, 28, 2748 (2016).

[5] J. Mu， S. A. Vázquez-Córdova， M. A. Sefunc, Y. S. Yong and S. M. GarcíaBlanco, "A low-loss and broadband MMI-based multi/demultiplexer in $\mathrm{Si}_{3} \mathrm{~N}_{4} / \mathrm{SiO}_{2}$ technology," Journal of Lightwave Technology, 34, 15, 3603 (2016).

\section{Contributions to conferences}

[1] Y. S. Yong, S. Aravazhi, S. A. Vázquez-Córdova, J. J. Carvajal, F. Díaz, J. L. Herek, S. M. García-Blanco, and M. Pollnau, "Temperature dependence of the transition cross sections of rare-earth ions," $18^{\text {th }}$ International Conference on Luminescence, Paraíba, Brazil, submitted (2017).

[2] Y. S. Yong, S. Aravazhi, S. A. Vázquez-Córdova, J. J. Carvajal, F. Díaz, J. L. Herek, S. M. García-Blanco, and M. Pollnau, "Direct confocal measurement of luminescence lifetimes on ground-state transitions with reabsorption," $18^{\text {th }}$ International Conference on Luminescence, Paraíba, Brazil, submitted (2017). 
[3] S. A. Vázquez-Córdova, S. Aravazhi, C. Grivas, Y. S. Yong, S. M. García-Blanco, J. L. Herek, and M. Pollnau, "High optical gain in $\mathrm{KGd}_{x} \mathrm{Lu}_{y} \mathrm{Er}_{1-x-y}\left(\mathrm{WO}_{4}\right)_{2}$ waveguide amplifiers despite energy-transfer upconversion," $18^{\text {th }}$ International Conference on Luminescence, Paraíba, Brazil, submitted (2017).

[4] Y. S. Yong, S. Aravazhi, S. A. Vázquez-Córdova, J. L. Herek, S. M. GarcíaBlanco, and M. Pollnau, "Temperature dependence of transition cross sections in rare-earth-doped laser materials," Conference on Lasers and Electro-Optics Europe, Munich, Germany, accepted (2017).

[5] S. A. Vázquez-Córdova, S. Aravazhi, C. Grivas, Y. S. Yong, S. M. García-Blanco, J. L. Herek, and M. Pollnau, "High gain in erbium-doped channel waveguides," Conference on Lasers and Electro-Optics Europe, Munich, Germany, accepted (2017).

[6] Y. S. Yong, S. A. Vázquez-Córdova, S. Aravazhi, C. Grivas, J. L. Herek, S. M. García-Blanco, and M. Pollnau, "High-gain waveguide amplifiers based on potassium rare-earth double tungstates," European Conference on Integrated Optics, Eindhoven, The Netherlands, paper T.5.1 (2017).

[7] Y. S. Yong, S. Aravazhi, S. A. Vázquez-Córdova, J. L. Herek, S. M. GarcíaBlanco, and M. Pollnau, "Temperature-dependent absorption and gain of ytterbium-doped potassium double tungstates for chip-scale amplifiers and lasers," Photonics West, San Francisco, USA, paper 10106-5 (2017).

[8] S. A. Vázquez-Córdova, S. Aravazhi, C. Grivas, A. M. Heuer, C. Kränkel, Y. S. Yong, S. M. García-Blanco, J. L. Herek, and M. Pollnau, "Spectroscopy of erbium-doped potassium double tungstate waveguides," Photonics West, San Francisco, USA, paper 10106-3 (2017).

[9] J. Mu, M. Dijkstra, M. de Geode, Y. S. Yong, S. M. García-Blanco, "Ultra-lowloss and broadband mode converters in $\mathrm{Si}_{3} \mathrm{~N}_{4}$ technology," Photonics West, San Francisco, USA, paper 10106-26, (2017).

[10] S. A. Vázquez-Córdova, S. Aravazhi, C. Grivas, Y. S. Yong, S. M. García-Blanco, J. L. Herek, and M. Pollnau, "13 dB/cm gain per unit length at $1.53 \mu \mathrm{m}$ in $\mathrm{KGd}_{\mathrm{x}} \operatorname{Lu}_{\mathrm{y}} \mathrm{Er}_{1-\mathrm{x}-\mathrm{y}}\left(\mathrm{WO}_{4}\right)_{2}$ channel waveguides, allowing for $\sim 40 \mathrm{~dB}$ total gain," Advanced Solid-State Lasers Congress, Boston, Massachusetts, paper ATu6A.3, post-deadline paper (2016). 
[11] Y. S. Yong, S. Aravazhi, S. A. Vázquez-Córdova, J. L. Herek, S. M. GarcíaBlanco, and M. Pollnau, "Influence of temperature on the transition cross-sections and the optical gain of highly $\mathrm{Yb}$-doped potassium double tungstates," Proc. $21^{\text {st }}$ Annu. Symp. IEEE Photonics Society Benelux Chapter, Ghent, Belgium, pp. 199202 (2016).

[12] S. A. Vázquez-Córdova, S. Aravazhi, C. Grivas, Y. S. Yong, S. M. García-Blanco, J. L. Herek, and M. Pollnau, "Er-doped potassium double tungstate channel waveguides with high optical gain," Proc. $21^{\text {st }}$ Annu. Symp. IEEE Photonics Society Benelux Chapter, Ghent, Belgium, pp. 47-50 (2016).

[13] J. Mu, M. Dijkstra, Y. S. Yong, S. A. Vázquez-Córdova, and S. M. GarcíaBlanco, "Low-loss highly tolerant vertical couplers for hybrid integration of $\mathrm{Si}_{3} \mathrm{~N}_{4}$ and multimode polymer waveguides", Proc. $21^{\text {st }}$ Annu. Symp. IEEE Photonics Society Benelux Chapter, Ghent, Belgium, pp. 271-274 (2016).

[14] S. A. Vázquez-Córdova, S. Aravazhi, C. Grivas, A. M. Heuer, C. Kränkel, Y. S. Yong, S. M. García-Blanco, J. L. Herek, and M. Pollnau, "Optical gain of $13 \mathrm{~dB} / \mathrm{cm}$ at $\sim 1.53 \mu \mathrm{m}$ in $\mathrm{KGd}_{\mathrm{x}} \mathrm{Lu}_{\mathrm{x}} \mathrm{Er}_{1-2 \mathrm{x}}\left(\mathrm{WO}_{4}\right)_{2}$ channel waveguides and the influence of parasitic spectroscopic processes," $7^{\text {th }}$ EPS-QEOD Europhoton Conference, Vienna, Austria, paper FWG-4.6 (2016).

[15] S. A. Vázquez-Córdova, Y. S. Yong, M. Dijkstra, J. L. Herek, S. M. GarcíaBlanco, and M. Pollnau, "Erbium doped waveguide amplifiers operating at the Cband”, Physics@FOM, Veldhoven, The Netherlands, paper P3.086 (2016).

[16] D. Geskus, Y. S. Yong, E.H. Bernhardi, L. Agazzi, S. M. García-Blanco, S. Aravazhi, and M. Pollnau, "The influence of high doping concentrations on the optical gain of $\mathrm{Yb}^{3+}$-doped potassium double tungstate channel waveguides," Optics \& Photonics in Sweden, Stockholm, Sweden (2015).

[17] S. A. Vázquez-Córdova, Y.S. Yong, J. L. Herek, S. M. García-Blanco, M. Pollnau, "Optical gain around $1.5 \mu \mathrm{m}$ in erbium-doped waveguide amplifiers," $17^{\text {th }}$ International Conference on Transparent Optical Networks, Budapest, Hungary, paper Th.A5.4, invited (2015).

[18] Y. S. Yong, S. Aravazhi, S. A. Vázquez-Córdova, S. M. García-Blanco, and M. Pollnau, " $1050 \mathrm{~dB} / \mathrm{cm}$ gain in a 57.5 at.\% $\mathrm{Yb}$-doped $\mathrm{KGd}\left(\mathrm{WO}_{4}\right)_{2}$ at $981 \mathrm{~nm}$," Conference on Lasers and Electro-Optics Europe, Munich, Germany, paper CE7.3 (2015). 
[19] D. Geskus, Y. S. Yong, E. H. Bernhardi, L. Agazzi, S. M. García-Blanco, S. Aravazhi, and M. Pollnau, "Concentration dependence of optical gain in $\mathrm{Yb}^{3+}$ doped potassium double tungstate channel waveguides," Conference on Lasers and Electro-Optics Europe, Munich, Germany, paper CJ-6a.3 (2015).

[20] Y. S. Yong, S. Aravazhi, S. A. Vázquez-Córdova, S. M. García-Blanco, and M. Pollnau, "Highly $\mathrm{Yb}$-doped $\mathrm{KGd}\left(\mathrm{WO}_{4}\right)_{2}$ thin-film amplifier," Conference on Lasers and Electro-Optics: Science \& Innovations, San José, CA, USA, paper STh1G.5 (2015).

[21] Y. S. Yong, S. Aravazhi, S. A. Vázquez-Córdova, J. L. Herek, S. M. GarcíaBlanco, and M. Pollnau, "Optical amplification in an Yb-doped potassium double tungstate thin film," Physics@FOM, Veldhoven, The Netherlands, paper P01.056 (2015).

[22] Y. S. Yong, S. Aravazhi, S. A. Vázquez-Córdova, S. M. García-Blanco, and M. Pollnau, "Gain characterisation of lattice-engineered potassium double tungstate thin-film with 57.5 at.\% ytterbium concentration for optical interconnects", Proc. 19 th Annu. Symp. IEEE Photonics Society Benelux Chapter, Enschede, The Netherlands, pp. 59-62 (2014).

[23] Y. S. Yong, S. A. Vázquez-Córdova, S. M. García-Blanco, S. Aravazhi, K. Wörhoff, and M. Pollnau, "Design of high-gain, small-footprint ytterbiumdoped potassium double tungstate waveguide amplifier for short-distance optical interconnects," Proc. 17 th Annu. Symp. IEEE Photonics Society Benelux Chapter, Mons, Belgium, pp. 239-242 (2012).

[24] S. A. Vázquez-Córdova, Y. S. Yong, S. Aravazhi, K. Wörhoff, and M. Pollnau, "Concentration quenching of luminescence lifetime in ytterbium-doped potassium double tungstate waveguide amplifiers," Proc. $17^{\text {th }}$ Annu. Symp. IEEE Photonics Society Benelux Chapter, Mons, Belgium, pp. 223-226 (2012).

\section{Other contributions}

[1] S. A. Vázquez-Córdova, Y. S. Yong, M. A. Sefunc, J. Mu, J. L. Herek, M. Pollnau, and S. M. García-Blanco, "Fabrication, characterization and integration of rare-earth doped waveguide amplifiers on different platforms," MESA+ Meeting 2014, Enschede, The Netherlands (2014).

[2] Y. S. Yong, K. van Dalfsen, D. Geskus, S. Aravazhi, S. A. Vázquez-Córdova, M. Sefunc, M. Dijkstra, K. Wörhoff, S. M. García-Blanco, and M. Pollnau, "Active devices on rare-earth-doped double tungstate and aluminum oxide material systems," MESA + Meeting 2013, Enschede, The Netherlands (2013). 
[3] K. van Dalfsen, D. Geskus, S. Aravazhi, M. Sefunc, Y. S. Yong, S. A. VázquezCórdova, M. Dijkstra, K. Wörhoff, S. M. García-Blanco, and M. Pollnau, "Lasers and amplifiers in double tungstate waveguides," MESA+ Meeting 2012, Enschede, The Netherlands (2012). 